ITS Forschungsberichte aus dem Institut für Thermische Strömungsmaschinen Hrsg.: Prof. Dr.-Ing. Hans-Jörg Bauer

Felix von Plehwe

Untersuchung des Wärmeübergangs durch Einspritzkühlung für ein Höchstleistungsgetriebe

Band 86/2021 



\title{
Untersuchung des Wärmeübergangs durch Einspritzkühlung für ein Höchstleistungsgetriebe
}

\author{
Zur Erlangung des akademischen Grades eines \\ DOKTORS DER INGENIEURWISSENSCHAFTEN (Dr.-Ing.) \\ der KIT-Fakultät für Maschinenbau des \\ Karlsruher Instituts für Technologie (KIT)
}

genehmigte

DISSERTATION

von

Dipl.-Ing. Felix C. von Plehwe

aus Scherzingen $(\mathrm{CH})$

Tag der mündlichen Prüfung:

12. Juli 2021

Hauptreferent:

Prof. Dr.-Ing. Hans-Jörg Bauer, Ord.

Korreferentin:

Prof. Dr.-Ing. habil. Andrea Luke 
Forschungsberichte aus dem

Institut für Thermische Strömungsmaschinen

herausgegeben von:

Prof. Dr.-Ing. Hans-Jörg Bauer,

Lehrstuhl und Institut für Thermische Strömungsmaschinen

Karlsruher Institut für Technologie (KIT)

Kaiserstr. 12

D-76131 Karlsruhe

Bibliografische Information der Deutschen Nationalbibliothek

Die Deutsche Nationalbibliothek verzeichnet diese Publikation in der Deutschen Nationalbibliografie; detaillierte bibliografische Daten sind im Internet über http://dnb.d-nb.de abrufbar.

\section{(1) $(1) \Theta(\Theta$}

CC Copyright Logos Verlag Berlin GmbH 2022

Alle Rechte vorbehalten.

ISSN $1615-4983$

ISBN 978-3-8325-5436-1

Logos Verlag Berlin GmbH

Georg-Knorr-Str. 4, Geb. 10,

12681 Berlin

Tel.: +4903042851090

Fax: +4903042851092

INTERNET: http://www.logos-verlag.de 


\section{Untersuchung des Wärmeübergangs durch Einspritzkühlung für ein Höchstleistungsgetriebe}

Dipl.-Ing. Felix C. von Plehwe 



\section{Vorwort des Herausgebers}

Der schnelle technische Fortschritt im Turbomaschinenbau, der durch extreme technische Forderungen und starken internationalen Wettbewerb geprägt ist, verlangt einen effizienten Austausch und die Diskussion von Fachwissen und Erfahrung zwischen Universitäten und industriellen Partnern. Mit der vorliegenden Reihe haben wir versucht, ein Forum zu schaffen, das neben unseren Publikationen in Fachzeitschriften die aktuellen Forschungsergebnisse des Instituts für Thermische Strömungsmaschinen am Karlsruher Institut für Technologie (KIT) einem möglichst großen Kreis von Fachkollegen aus der Wissenschaft und vor allem auch der Praxis zugänglich macht und den Wissenstransfer intensiviert und beschleunigt.

Flugtriebwerke, stationäre Gasturbinen, Turbolader und Verdichter sind im Verbund mit den zugehörigen Anlagen faszinierende Anwendungsbereiche. Es ist nur natürlich, dass die methodischen Lösungsansätze, die neuen Messtechniken, die Laboranlagen auch zur Lösung von Problemstellungen in anderen Gebieten - hier denke ich an Otto- und Dieselmotoren, elektrische Antriebe und zahlreiche weitere Anwendungen - genutzt werden. Die effiziente, umweltfreundliche und zuverlässige Umsetzung von Energie führt zu Fragen der ein- und mehrphasigen Strömung, der Verbrennung und der Schadstoffbildung, des Wärmeübergangs sowie des Verhaltens metallischer und keramischer Materialien und Verbundwerkstoffe. Sie stehen im Mittelpunkt ausgedehnter theoretischer und experimenteller Arbeiten, die im Rahmen nationaler und internationaler Forschungsprogramme in Kooperation mit Partnern aus Industrie, Universitäten und anderen Forschungseinrichtungen durchgeführt werden.

Es sollte nicht unerwähnt bleiben, dass alle Arbeiten durch enge Kooperation innerhalb des Instituts geprägt sind. Nicht ohne Grund ist der Beitrag der Werkstätten, der Technik-, der Rechnerund Verwaltungsabteilungen besonders hervorzuheben. Diplomanden und Hilfsassistenten tragen mit ihren Ideen Wesentliches bei, und natürlich ist es der stets freundschaftlich fordernde wissenschaftliche Austausch zwischen den Forschergruppen des Instituts, der zur gleichbleibend hohen Qualität der Arbeiten entscheidend beiträgt. Dabei sind wir für die Unterstützung unserer Förderer außerordentlich dankbar.

Die in jüngster Zeit in ersten Turbofantriebwerken vollzogene Änderung der Triebwerksarchitektur, die Integration eines Planetengetriebes zwischen dem Gebläse (Fan) des Triebwerks und der Niederdruckwelle, führt zu einer drastischen Reduktion des Brennstoffverbrauchs in der Größenordnung von 10 - 15\% im Vergleich zur vorigen Triebwerksgeneration. Eine wesentliche Herausforderung bei der Auslegung des Untersetzungsgetriebes von Triebwerken für GroßraumLangstreckenflugzeuge besteht in der Beherrschung seines Thermalhaushalts. Aufgrund der immensen übertragenen Leistung führen selbst geringe relative mechanische Verluste des Getriebes zur Dissipation einer Leistung von mehreren hundert Kilowatt. Die dissipierte Leistung ist durch eine effektive und effiziente Kühlung der Zahnräder aus dem Getriebe abzuleiten. Dabei steht die Kühlung, die durch Besprühen der Zahnflanken mit Ölstrahlen erfolgt, vor der Herausforderung, eine ausreichende Temperaturabsenkung der Zahnräder sicherzustellen und gleichzeitig aber die eingesetzte Ölmenge zu begrenzen, um keine zusätzlichen Planschverluste zu generieren. Um dieses Ziel erreichen zu können, ist die genaue Kenntnis des Wärmeüber- 
gangs zwischen Ölstrahl und Zahnflanke unbedingte Voraussetzung. Im vorliegenden Band der Schriftenreihe befasst sich der Autor folgerichtig mit der experimentellen Untersuchung des Wärmeübergangs zwischen Ölstrahlen und den Flanken eines repräsentativen Zahnrads. Die Ergebnisse der ortsaufgelösten Messungen des Wärmeübergangs ermöglichen, die Wirkzusammenhänge zwischen den Randbedingungen Ölvolumenstrom, Drehzahl, Düsenwinkel, Strahleindringtiefe und Aufprallwinkel und dem mittleren Wärmeübergang zu identifizieren und physikalisch verständlich zu erklären. Im Gegensatz zu bisher verwendeten Korrelationen zur Abschätzung des Wärmeübergangs wird erstmals der dominierende Effekt der Prallkühlung des auf die Zahnflanke auftreffenden Ölstrahls erfasst. Die hohe Qualität der aus den Messungen abgeleiteten Korrelation für den mittleren Wärmeübergang ermöglicht nun eine erheblich sicherere wärmetechnische Auslegung von Hochleistungsgetrieben für zukünftige Flugtriebwerke mit gesteigerten Nebenstromverhältnissen und höchsten Vortriebswirkungsgraden.

Karlsruhe, im September 2021

Hans-Jörg Bauer 


\section{Vorwort des Autors}

Die vorliegende Arbeit ist während meiner Mitarbeit am Institut für Thermische Strömungsmaschinen (ITS) am Karlsruher Institut für Technologie (KIT) entstanden. Das zugrunde liegende Forschungsprojekt zur Untersuchung der Einspritzkühlung von Leistungsgetrieben in Flugtriebwerken wurde in enger Zusammenarbeit mit Rolls-Royce Deutschland im Rahmen des Luftfahrtforschungsprogramms des Bundesministeriums für Wirtschaft und Energie (BMWi) durchgeführt.

Im Gegensatz zu der linearen, deterministischen und kühlen Form, die das Endprodukt Dissertation naturgemäß annimmt, ist ihre Entstehungsgeschichte verwinkelt, zunächst uneindeutig und bisweilen auch hitzig. Dabei traten einige Hürden auf, die in den Kapiteln dieser Arbeit unerwähnt bleiben. Die Überwindung dieser Hürden wurde erst durch meine Unterstützer und Mitstreiter möglich, denen ich an dieser Stelle danken möchte.

Herrn Professor Hans-Jörg Bauer danke ich dafür, am ITS arbeiten zu dürfen und für die Betreuung meiner Arbeit. Wie auch zahlreichen Vorworten dieser Schriftenreihe zu entnehmen ist, stellt die Einbindung in die vielfältigen akademischen Aktivitäten des Instituts eine hervorragende Möglichkeit dar, sich fachlich weiterzuentwickeln. Eine aus meiner Sicht besonders wertvolle Lehre entstammt jedoch der Arbeit an industrienahen Forschungsprojekten des ITS. In diesen Projekten wurden mir große Freiheiten zuteil, sowohl in der Organisation als auch in der wissenschaftlichen und technischen Herangehensweise. Die daraus hervorgehende Verantwortung bringt eine professionelle Weiterentwicklung mit sich, von der ich heute besonders profitiere. In Anbetracht dessen, dass es sich um Industrieprojekte mit entsprechenden Ansprüchen der Auftraggeber handelt, bin ich Herrn Professor Bauer für das mir entgegengebrachte Vertrauen besonders dankbar.

In ähnlicher Weise möchte ich Frau Dr. Corina Schwitzke, in deren Forschungsgruppe ich arbeiten durfte, danken. Neben ausgezeichneter fachlicher Kompetenz besitzt Frau Dr. Schwitzke selbst in stressigen, schwierigen Situationen die Gelassenheit einer Jazzband und gleichzeitig die Organisiertheit eines Raumfahrtprogramms. Diese einzigartige Qualität strahlt auf alle Beteiligten aus, wodurch die Arbeit am Institut nicht nur gut funktioniert, sondern auch große Freude bereitet.

Frau Professorin Andrea Luke danke ich für die Übernahme des Korreferats und das entgegengebrachte Interesse an meiner Arbeit. Herrn OStD Peter Labouvie danke ich für das Lektorat meiner Arbeit und die interessanten Diskussionen zu wissenschaftlicher Sprache.

Experimentelle Ergebnisse stellen einen wesentlichen Teil dieser Arbeit dar. Die Versuche hinter diesen Ergebnissen waren stark von Murphys Gesetz betroffen - was schief gehen kann, wird schief gehen. Im wiederholten Aufbau und Betrieb, der Fehlersuche und eben häufig auch Reparatur der Prüfstände konnte ich glücklicherweise auf das breit aufgestellte Team der technischen Mitarbeiter des ITS bauen. Ihre große Fähigkeit und Erfahrung haben sich in Verbindung mit der badischen Herzlichkeit dieses Teams als unschlagbares Gegenmittel zu Murphys Gesetz erwiesen. Für diese Unterstützung gilt ihnen mein besonderer Dank. 
Der wissenschaftliche Austausch mit den Doktoranden des Instituts führte regelmäßig zu neuen Ideen und verbesserten Lösungen für Problemstellungen. Aber auch für mein Leben in Karlsruhe war dieses Umfeld ein großer Zugewinn, wofür ihnen mein herzlicher Dank gebührt. Hervorheben möchte ich einerseits Herrn Dr. Maximilian Elfner, mit dem ich über lange Zeit ein Büro teilte. Seine kreativen Ideen und sein herausragendes Umsetzungsvermögen haben meiner Arbeit zahlreiche wichtige Impulse gegeben. Andererseits Herrn Dr. Franz Pütz, der unter den Doktoranden stets neue (gerne ökonomisch-wirtschaftliche) Blickwinkel eingebracht und mein Leben in mehrfacher Hinsicht bereichert hat.

Besonders hervorheben möchte ich meine Familie, die meinen Weg weit über das hier Darstellbare hinaus geprägt hat. Meine Lebensgefährtin Constanze, für unzählige fruchtvolle Diskurse, Impulse und Erdungen in einem bunten Spektrum an Themen. Meine Eltern, für die vielen richtigen Anregungen und die beständige Unterstützung in meinen Entscheidungen. Meine Schwester Caroline, die in der gesamten Bandbreite von entscheidendsten Problemen bis zu amüsanten Nichtigkeiten eine Wellenlänge mit mir teilt.

München, im Dezember 2021

Felix von Plehwe 


\section{Inhaltsverzeichnis}

Abbildungsverezichnis $\quad$ xi

Tabellenverzeichnis $\quad$ Xv

Symbolverzeichnis $\quad$ xvii

1 Einführung 1

2 Grundlagen und wissenschaftlicher Kenntnisstand 3

2.1 Getriebemechanik . . . . . . . . . . . . . . . . . . 4

2.1.1 Reibung und Verluste . . . . . . . . . . . . . . . . 4

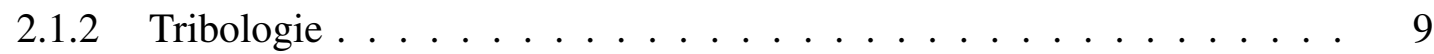

2.2 Thermalmodellierung von Zahnrädern . . . . . . . . . . . . . . . 15

2.3 Prallkühlung an Zahnrädern _ . . . . . . . . . . . . . . . . . . . . . . . . 19

2.4 Wärmeübergang durch Prallkühlung . . . . . . . . . . . . . . . 22

2.4.1 Prallkühlung durch Flüssigkeitsstrahlen . . . . . . . . . . . . . 24

2.4.2 Prallkühlung an bewegten Oberflächen . . . . . . . . . . . 26

2.4 .3 Instationäre Prallkühlung . . . . . . . . . . . . . . . . . 27

2.4 .4 Zusammenfassung . . . . . . . . . . . . . . . 28

2.5 Zielsetzung dieser Arbeit . . . . . . . . . . . . . . . . . . . . . . 29

3 Untersuchte Öleinspritzkühlung 31

3.1 Öleinspritzkühlung . . . . . . . . . . . . . . . 31

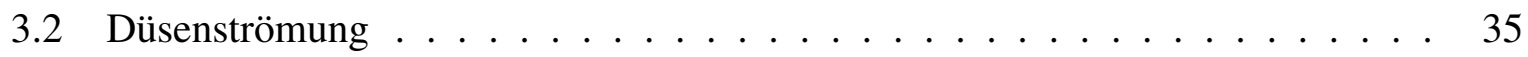

3.3 Kinematik des Ölstrahls . . . . . . . . . . . . . . . . . . . . . 38

4 Experimentelle Methode zur Bestimmung des Öl-Zahnrad-Wärmeübergangs 45

4.1 Erzeugung des Wärmeübergangs . . . . . . . . . . . . . . 45

4.2 Modellvereinfachung zur Erhöhung der Auflösung . . . . . . . . . . . . . . 48

4.3 Iterative Berechnung des Wärmeübergangs . . . . . . . . . . . . . 50

4.4 Experimentelle Umsetzung . . . . . . . . . . . . . . . . . . . . . . 52

4.4.1 Optimierte Gestaltung eines Versuchszahnrads . . . . . . . . . . 52

4.4.2 Instrumentierung des Versuchszahnrads . . . . . . . . . . . . . . 54 
4.5 Analyse der Messgenauigkeit . . . . . . . . . . . . . . . . 58

4.5.1 Monte-Carlo-Methode . . . . . . . . . . . . . . . . . 59

4.5.2 Unsicherheit der Wärmeübergangsmessungen . . . . . . . . . . . . . 61

4.5.3 Zusammenfassung ........................ 65

$\begin{array}{lll}5 & \text { Versuchsaufbau und Messtechnik } & 67\end{array}$

5.1 Implementierung in einem Versuchsstand . . . . . . . . . . . . . . . 67

5.2 Anlagen zur Ölversorgung und Luftkühlung . . . . . . . . . . . . . . . 70

5.3 Messtechnik . . . . . . . . . . . . . . . . . . 71

6 Ergebnisse $\quad 73$

6.1 Messung und Auswertung des Wärmeübergangs . . . . . . . . . . . . . . . . 74

6.2 Charakterisierung des Öl-Zahnrad-Wärmeübergangs _ . . . . . . . . . . 78

6.2.1 Einfluss der Drehzahl und des Ölvolumenstroms . . . . . . . . . . . 79

6.2.2 Einfluss des Düsenwinkels . . . . . . . . . . . . . . . . . 89

6.2.3 Einfluss der Temperatur . . . . . . . . . . . . . . . 96

6.3 Ableitung einer Korrelation aus den gewonnenen Daten . . . . . . . . . . . . 98

$\begin{array}{lll}7 & \text { Zusammenfassung } & 103\end{array}$

$\begin{array}{ll}\text { Anhang } & 105\end{array}$

A.1 Gemessene Betriebspunkte und Wärmeübergangsverteilungen . . . . . . . . 105

A.1.1 Tabellarische Darstellung der Parameter . . . . . . . . . . . . . . . . 105

A.1.2 Ortsaufgelöste Wärmeübergangskoeffizienten . . . . . . . . . . . . 107

A.2 Stoffwertkorrelationen des verwendeten Öls . . . . . . . . . . . . . . . 130

A.3 Herleitung des spezifischen Relativimpulses . . . . . . . . . . . . . 130

$\begin{array}{ll}\text { Literaturverzeichnis } & 133\end{array}$ 


\section{Abbildungsverzeichnis}

2.1 Schematische Darstellung des Eingriffs zweier evolventischer Zahnräder und der auftretenden Geschwindigkeiten nach Klocke und Brecher (2017) . . . . 5

2.2 Druckverteilung im Schmierspalt nach Hertzscher und elastohydrodynamischer Theorie und charakteristischer EHD-Schmierfilm nach Theißen (2003) . . . . . 10

2.3 Einfluss der Oberflächentemperatur vor dem Eingriff auf die minimale relative Schmierfilmdicke . . . . . . . . . . . . . . . . . . . 12

2.4 Schmierfilmzustände im Zahnradeingriff nach Spikes (1997) _ . . . . . . . . 13

2.5 Einfluss der spezifischen Schmierfilmdicke $\lambda_{\mathrm{GF}}$ auf die Lebensdauer nach Krantz (2014) . . . . . . . . . . . . . . . . . . . . . 14

2.6 Periodisches Zahnelement und Wärmeübergangsgebiete nach Patir und Cheng

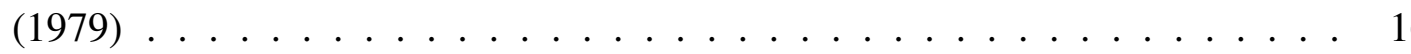

2.7 Isolinien der Temperatur bei dominierendem Seitenwandwärmeübergang (links) und dominierendem Zahnflankenwärmeübergang (rechts) nach Patir und Cheng

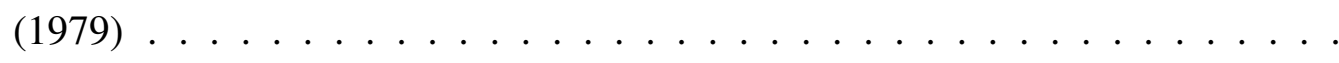

2.8 Auszentrifugieren eines Fluidfilms in einem Zahnquadranten nach DeWinter und Blok (1974) . . . . . . . . . . . . . . . . . . 20

2.9 Einfluss des Düsenvordrucks und des übertragenen Moments auf die Zahnradtemperatur nach Townsend und Akin (1983) . . . . . . . . . . . . 21

2.10 Ausbildung einer Grenzschicht in der Stauströmung durch Prallkühlung mit einem unbewegten, flüssigen Rundstrahl in Gasumgebung nach Lienhard (2006) 24

2.11 Unterteilung eines auftreffenden Prallstrahls in charakteristische Strömungsgebiete nach Liu et al. (1991) . . . . . . . . . . . . . . . . 25

2.12 Verteilung des Wärmeübergangs durch intermittierende Prallkühlung bei unterschiedlichen Unterbrechungsfrequenzen nach Zumbrunnen und Aziz (1993) . . 28

3.1 Charakteristische geometrische Parameter der untersuchten Öleinspritzkühlung

3.2 Verlauf der Zerfallslänge eines Ölstrahls in Abhängigkeit der Strömungsgeschwindigkeit nach Lin und Reitz (1998) . . . . . . . . . . . . . . . 36

3.3 Anliegende und vollständig abgelöste Düsenströmungen nach von Kuensberg Sarre et al. (1999) . . . . . . . . . . . . . . . . . 37

3.4 Kinematisches Ölaufprallmodell nach Akin et al. (1975) . . . . . . . . . . . 39

3.5 Kinematik des Ölstrahls unter Berücksichtigung des Düsenwinkels . . . . . . . 40

3.6 Diskrete Werte des Aufprallwinkels an vier signifikanten Stellen der Flanke . . 40

3.7 Einfluss des Düsenwinkels auf den Aufprallwinkel $\beta$ für verschiedene Geschwindigkeitsverhältnisse $\Sigma \ldots \ldots \ldots \ldots$. . . . . . . . . . . . . 42 
3.8 Einfluss des Düsenwinkels auf den Relativimpuls $\frac{u_{\mathrm{r}}}{u_{\mathrm{z}}}=\sqrt{1+\Sigma^{2}-2 \Sigma \sin (\alpha)}$ für verschiedene Geschwindigkeitsverhältnisse $\Sigma \ldots \ldots \ldots$

4.1 Qualitative Temperaturverteilung eines Zahnradsektors mit vereinfachten thermischen Randbedingungen . . . . . . . . . . . . . . . . . . .

4.2 Vereinfachung des Zahnrads als Viertelzahn - Symmetrieebenen und periodische Ränder

4.3 Schematische Darstellung der iterativen Berechnung des Wärmeübergangs aus diskreten Temperaturmesswerten . . . . . . . . . . . . . . . . . . 52

4.4 Eindimensionale Betrachtung des Einzelzahns . . . . . . . . . . . . . 54

4.5 Verlauf der Temperaturen an den beiden Oberflächen des Ersatzkörpers, $\vartheta$ und $\vartheta_{\mathrm{K}}$, über einen Bereich von $h$ für verschiedene Prüfkörperdicken $L$ in $\mathrm{m}$. . . . 55

4.6 Flächentreues, abgewickeltes $(s, y)$ - Koordinatensystem eines Einzelzahns . . . 55

4.7 Verteilung der Messpunkte über einen Viertelzahn im $\left(s^{+}, y^{+}\right)$-Koordinatensystem 56

4.8 In Nut eingeklebtes Mantelthermoelement . . . . . . . . . . . . . . . . . 57

4.9 Fehlerfortpflanzungsanalyse durch die Monte-Carlo-Methode nach Wübbeler et al. (2008) . . . . . . . . . . . . . . . . . . . 60

4.10 Angenommener Wärmeübergang und berechnete Temperaturverteilung für die Monte-Carlo-Simulation . . . . . . . . . . . . . . . . 61

4.11 Messunsicherheit des mittleren Wärmeübergangs _ . . . . . . . . . . . 62

4.12 Abhängigkeit des lokalen Wärmeübergangskoeffizienten von der Messunsicherheit der Öltemperatur . . . . . . . . . . . . . . . . . . . . . . . . . . 63

4.13 Unsicherheit des lokalen Wärmeübergangs an der Messposition 9 . . . . . . 63

4.14 Korrelationskoeffizienten des Wärmeübergangs an Position 9 und der Temperaturen an den Messpunkten . . . . . . . . . . . . . . . . . . . . . . 64

4.15 Korrelationskoeffizienten aller lokalen Wärmeübergangskoeffizienten von allen Oberflächentemperauren . . . . . . . . . . . . . . . . . . . 64

5.1 Schematische Darstellung des eingesetzten Versuchsstands zur Messung des Öl-Zahnrad-Wärmeübergangs . . . . . . . . . . . . . . . . . . 68

5.2 Schnittdarstellung der Ölbrause mit Zuführbohrung durch die Düsenebene . . . 69

5.3 Anlagenplan des eingesetzten Versuchsstands zur Messung des Öl-ZahnradWärmeübergangs . . . . . . . . . . . . . . . . . . 70

6.1 Verteilung der untersuchten Betriebspunkte im Parameterraum . . . . . . . . 73

6.2 Exemplarischer Verlauf der Betriebsparameter und Temperaturen während einer Messung . . . . . . . . . . . . . . . . . 75

6.3 Gemessene Temperaturen auf der Zahnkontur für BP80 . . . . . . . . . . . 76 
6.4 Wärmeübergangsverteilung für BP80 in $\mathrm{W} /\left(\mathrm{m}^{2} \mathrm{~K}\right) \ldots \ldots \ldots$

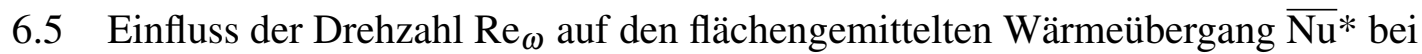
$\operatorname{Re}_{d}=2639$ und den ortsaufgelösten Wärmeübergang $\mathrm{Nu} \ldots \ldots$. . . . . . 80

6.6 Gegenlichtaufnahmen bei $\operatorname{Re}_{d}=2639 \ldots \ldots \ldots$. . . . . . . . 81

6.7 Einfluss der Drehzahl $\operatorname{Re}_{\omega}$ auf den flächengemittelten Wärmeübergang $\overline{\mathrm{Nu}}^{*}$ bei $\mathrm{Re}_{d}=1757$ und den ortsaufgelösten Wärmeübergang $\mathrm{Nu} \ldots \ldots$. . . . . . 84

6.8 Einfluss der Drehzahl $\operatorname{Re}_{\omega}$ auf den flächengemittelten Wärmeübergang $\overline{\mathrm{Nu}}^{*}$ bei $\mathrm{Re}_{d}=3954$ und den ortsaufgelösten Wärmeübergang $\mathrm{Nu} \ldots \ldots$. . . . . . . 87

6.9 Verlauf von $\overline{\mathrm{Nu}}^{*}$ in Abhängigkeit von $\operatorname{Re}_{\omega}$ und $\operatorname{Re}_{d}$ und für $\alpha=20^{\circ} \ldots$. . . 88

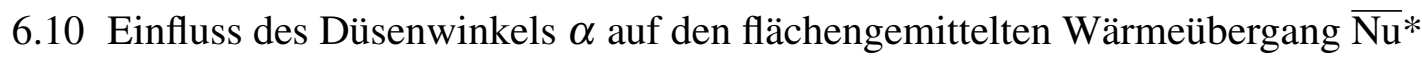
bei $\operatorname{Re}_{d}=2639 \ldots \ldots \ldots$. . . . . . . . . . . . . . . 90

6.11 Ortsaufgelöste Darstellung des Wärmeübergangs $\mathrm{Nu}$ bei $\mathrm{Re}_{d}=2639$ und hohen Drehzahlen ........................... 91

6.12 Ortsaufgelöste Darstellung des Wärmeübergangs $\mathrm{Nu}$ bei $\mathrm{Re}_{d}=2639$ und niedrigen Drehzahlen . . . . . . . . . . . . . . . . . . . 93

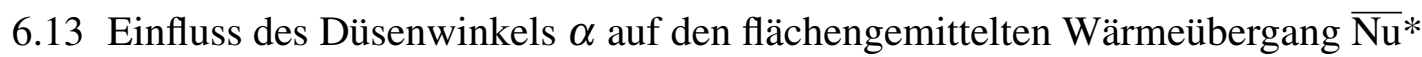
bei $\operatorname{Re}_{d}=2639$ und $\operatorname{Re}_{\omega}=143 \cdot 10^{4} \ldots \ldots$. . . . . . . . . . . . . 94

6.14 Einfluss des Düsenwinkels $\alpha$ auf die Wärmeübergangsverteilung $\mathrm{Nu}$ bei $\operatorname{Re}_{d}=$ 2639 und $\operatorname{Re}_{\omega}=143 \cdot 10^{4} \ldots \ldots \ldots \ldots$. . . . . . . . . . . . 95

6.15 Einfluss der Drehzahl $\operatorname{Re}_{\omega}$ auf den flächengemittelten Wärmeübergang $\overline{\mathrm{Nu}}^{*}$ bei $\operatorname{Re}_{d}=1619$ und den ortsaufgelösten Wärmeübergang $\mathrm{Nu}$ bei reduzierter Öltemperatur $\vartheta_{\mathrm{s}}=60^{\circ} \mathrm{C} \ldots \ldots$. . . . . . . . . . . . . . 97

6.16 Werte des Ausdrucks $\bar{h} /\left(u_{\mathrm{r}}\right)^{0,5}$ in Abhängigkeit von $\beta$ für alle gemessenen Betriebspunkte bei $\vartheta_{\mathrm{s}}=80^{\circ} \mathrm{C} \ldots \ldots \ldots$. . . . . . . . . . . 99

6.17 Vergleich der gemessenen und der durch die Korrelation berechneten mittleren Wärmeübergangskoeffizienten, jeweils bezogen auf den maximalen gemessenen mittleren Wärmeübergangskoeffizienten $\bar{h}_{\max } \ldots \ldots$. . . . . . . . . 100

A.1 Ortsaufgelöste Wärmeübergangsmessungen für BP1 bis BP6 . . . . . . . . 108

A.2 Ortsaufgelöste Wärmeübergangsmessungen für BP7 bis BP12 . . . . . . . . . 109

A.3 Ortsaufgelöste Wärmeübergangsmessungen für BP13 bis BP18 . . . . . . . . . 110

A.4 Ortsaufgelöste Wärmeübergangsmessungen für BP19 bis BP24 . . . . . . . . . 111

A.5 Ortsaufgelöste Wärmeübergangsmessungen für BP25 bis BP30 . . . . . . . . 112

A.6 Ortsaufgelöste Wärmeübergangsmessungen für BP31 bis BP36 . . . . . . . . . 113

A.7 Ortsaufgelöste Wärmeübergangsmessungen für BP37 bis BP42 . . . . . . . . . 114

A.8 Ortsaufgelöste Wärmeübergangsmessungen für BP43 bis BP48 . . . . . . . . . 115 
A.9 Ortsaufgelöste Wärmeübergangsmessungen für BP49 bis BP54 . . . . . . . . . 116

A.10 Ortsaufgelöste Wärmeübergangsmessungen für BP55 bis BP60 . . . . . . . . . 117

A.11 Ortsaufgelöste Wärmeübergangsmessungen für BP61 bis BP66 . . . . . . . . . 118

A.12 Ortsaufgelöste Wärmeübergangsmessungen für BP67 bis BP72 . . . . . . . . . 119

A.13 Ortsaufgelöste Wärmeübergangsmessungen für BP73 bis BP78 . . . . . . . . . 120

A.14 Ortsaufgelöste Wärmeübergangsmessungen für BP79 bis BP84 . . . . . . . . . 121

A.15 Ortsaufgelöste Wärmeübergangsmessungen für BP85 bis BP90 . . . . . . . . . 122

A.16 Ortsaufgelöste Wärmeübergangsmessungen für BP91 bis BP96 . . . . . . . . . 123

A.17 Ortsaufgelöste Wärmeübergangsmessungen für BP97 bis BP102 . . . . . . . . 124

A.18 Ortsaufgelöste Wärmeübergangsmessungen für BP103 bis BP108 . . . . . . . 125

A.19 Ortsaufgelöste Wärmeübergangsmessungen für BP109 bis BP114 . . . . . . . 126

A.20 Ortsaufgelöste Wärmeübergangsmessungen für BP115 bis BP120 . . . . . . . 127

A.21 Ortsaufgelöste Wärmeübergangsmessungen für BP121 bis BP126 . . . . . . . 128

A.22 Ortsaufgelöste Wärmeübergangsmessungen für BP127 bis BP129 . . . . . . . 129 


\section{Tabellenverzeichnis}

2.1 Bewertung der spezifischen Schmierfilmdicke $\lambda_{\mathrm{GF}} \ldots \ldots \ldots$

2.2 Literaturstellen zur Thermalmodellierung von Zahnrädern . . . . . . . . . . . 15

3.1 Geometrische Parameter der untersuchten Einspritzkühlung . . . . . . . . . . 33

3.2 Geometrie des untersuchten Zahnrads . . . . . . . . . . . . . . . . . 33

3.3 Wertebereich der Untersuchungen . . . . . . . . . . . . . 35

4.1 Positionen der Messpunkte und Verteilung über das Zahnrad . . . . . . . . . 58

5.1 Betriebsgrenzen des Versuchsstands . . . . . . . . . . . . . . . . 68

5.2 Genauigkeit . . . . . . . . . . . . . . . . . 72

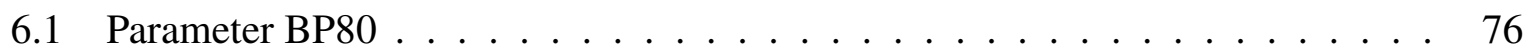

6.2 Polynomkoeffizienten des stückweisen Polynomansatzes nach Gleichung 6.6 100

A.1 Parameter und $\overline{\mathrm{Nu}}^{*}$ der gemessenen Betriebspunkte . . . . . . . . . . . . 105 


\section{Symbolverzeichnis}

\section{Dimensionslose Kennzahlen}

Bi Biotzahl, $\mathrm{Bi}=\frac{h L_{\mathrm{Bi}}}{\lambda}$

$E^{\prime} \quad$ Effektiver E-Modul, $E^{\prime}=\frac{E}{1-v^{2}}$

$G \quad$ Materialparameter der Elastohydrodynamik, $G=\alpha E^{\prime}$

$\mathrm{Nu} \quad \mathrm{Nußeltzahl,} \mathrm{Nu}=\frac{h d}{\lambda}$

Pr Prandtlzahl, $\operatorname{Pr}=\frac{v}{a}$

Re Reynoldszahl, $\operatorname{Re}_{d}=\frac{u_{\mathrm{s}} d}{v}$

$U \quad$ Geschwindigkeitsparameter der Elastohydrodynamik, $U=\frac{\eta_{0} u}{E^{\prime} R}$

$W \quad$ Lastparameter der Elastohydrodynamik, $W=\frac{w}{E^{\prime} R}$

\section{Lateinische Symbole}

a Temperaturleitfähigkeit

$A_{d} \quad$ Düsenquerschnitt

$\mathrm{m}^{2} / \mathrm{s}$

$b \quad$ Zahnbreite

$\mathrm{m}^{2}$

$c \quad$ Spezifische Wärmekapazität

$d \quad$ Düsendurchmesser

$\mathrm{J} /(\mathrm{kgK})$

$d_{\mathrm{w}}$

Teilkreisdurchmesser

$\mathrm{m}$

E

E-Modul

$\mathrm{m}$

E

Exzentrizität des Düsenauslasses

$\mathrm{Pa}$

$F_{\mathrm{n}} \quad$ Normalkraft

$\mathrm{mm}$

$F_{\mathrm{R}}$

Reibkraft

$\mathrm{N}$

Eingriffstrecke

$\mathrm{N}$

$g$

$\mathrm{m}$

$h \quad$ Wärmeübergangskoeffizient

$\mathrm{W} /\left(\mathrm{m}^{2} \mathrm{~K}\right)$

$h_{\min }$

Minimale Schmierfilmdicke

$\mathrm{m}$

$K_{\mathrm{A}} \quad$ Belastungsabhängiger Anwendungsfaktor

$L \quad$ Charakteristische Länge

$l \quad$ Eingriffslänge

$\mathrm{m}$

$l$

Länge der Düsenbohrung

$\mathrm{m}$

$m \quad$ Modul

$n \quad$ Drehzahl

$\mathrm{mm}$

$\vec{n} \quad$ Normalenvektor

$p_{\mathrm{e}}$

Eingriffsteilung

$\mathrm{m}$

$P_{\mathrm{VB} 0}$

Lastunabhängige Lagerverluste

$P_{\mathrm{VB}}$

Lastabhängige Lagerverluste

$P_{\mathrm{VD}}$

Dichtungsverluste

W

$P_{\mathrm{VX}}$

Sonstige Verluste

W

$P_{\mathrm{Vz} 0} \quad$ Lastunabhängige Verzahnungsverluste

W

$P_{\mathrm{Vz}}$

Lastabhängige Verzahnungsverluste 


$\begin{array}{llr}P_{\mathrm{V}} & \text { Gesamtverlust } & \mathrm{W} \\ q & \text { Spezifische Wärme } & \mathrm{J} / \mathrm{m}^{2} \\ \dot{q} & \text { Wärmestromdichte } & \mathrm{W} / \mathrm{m}^{2} \\ q_{\mathrm{V}} & \text { Volumetrische Wärmestromdichte } & \mathrm{W} / \mathrm{m}^{3} \\ R & \text { Düsenradius } & \mathrm{m} \\ R_{1}, R_{2} & \text { Krümmungsradius der Wälzpartner } & \mathrm{m} \\ R_{\mathrm{a}} & \text { Arithmetischer Mittenrauwert } & \mathrm{m} \\ s & \text { Konturkoordinate } & \mathrm{m} \\ s^{+} & \text {Dimensionslose Konturkoordinate } & - \\ t & \text { Zeit } & \mathrm{s} \\ T_{0} & \text { Zahntemperatur im beginnenden Eingriff } & \mathrm{K} \\ u & \text { Oberflächengeschwindigkeit } & \mathrm{m} / \mathrm{s} \\ u_{\mathrm{r}} & \text { Relativgeschwindigkeit } & \mathrm{m} / \mathrm{s} \\ u_{\mathrm{S}} & \text { Strahlgeschwindigkeit } & \mathrm{m} / \mathrm{s} \\ u_{\mathrm{z}} & \text { Tangentialgeschwindigkeit des Zahns } & \mathrm{m} / \mathrm{s} \\ V & \text { Ölvolumenstrom } & \mathrm{m} / \mathrm{s} \\ v_{\mathrm{g} 1}, v_{\mathrm{g} 2} & \text { Gleitgeschwindigkeit } & \mathrm{m} / \mathrm{s} \\ v_{\Sigma \mathrm{m}} & \text { Mittlere Summengeschwindigkeit } & \mathrm{m} / \mathrm{s} \\ W & \text { Zahnbreite } & \mathrm{m} \\ w & \text { Eingriffslängenbezogene Normalkraft } & \mathrm{N} / \mathrm{m} \\ X_{\mathrm{R}} & \text { Rauigkeitsfaktor } & - \\ y & \text { Axiale Koordinate } & \mathrm{m} \\ y^{+} & \text {Dimensionslose axiale Koordinate } & - \\ Z & \text { Zähnezahl } & - \\ z & \text { Anzahl ausgewerteter Messpunkte } & -\end{array}$

\section{Griechische Symbole}

$\alpha$

$\alpha$

$\beta$

$\varepsilon$

$\eta_{0}$

$\gamma$

$\imath$

$\lambda$

$\lambda_{\mathrm{GF}}$

$\mu$

$v$

$v$

$\omega$

$\rho$

$\rho_{\mathrm{m}}$

Druck-Viskositätskoeffizient

$\mathrm{m}^{2} / \mathrm{N}$

Düsenwinkel

Aufprallwinkel

Emissionsgrad

Viskosität bei Zahntemperatur

$\mathrm{kg} /(\mathrm{ms})$

Eingriffswinkel

Schrägungswinkel

Wärmeleitfähigkeit

$\mathrm{W} /(\mathrm{m} \mathrm{K})$

Spezifische Schmierfilmdicke

Reibwert

Kinematische Viskosität

$\mathrm{m}^{2} / \mathrm{s}$

Querkontraktionszahl

$\mathrm{rad} / \mathrm{s}$

Drehzahl

$\mathrm{kg} / \mathrm{m}^{3}$

Dichte

Mittlerer Ersatzkrümmungsradius

$\mathrm{m}$ 
$\Sigma \quad$ Geschwindigkeitsverhältnis

$\vartheta_{\mathrm{s}} \quad$ Öltemperatur K

$\xi \quad$ Verhältnis aus Segmentbreite und Düsendurchmesser

\section{Indizes}

$\omega$

Drehzahl- oder rotationsbezogen

$d \quad$ Bezogen auf den Düsendurchmesser

kond Konduktiv

konv Konvektiv

$\max \quad$ Maximal

$\min \quad$ Minimal

r Radialkomponente

$\mathrm{t}$ Tangentialkomponente 


\section{Einführung}

Eine der stärksten Triebfedern der Forschung im Bereich der Luftfahrt im Allgemeinen und der Turbomaschinen im Speziellen ist zweifellos das Streben nach besserer Umweltverträglichkeit des Luftverkehrs. Aus Gründen des Wettbewerbs und durch politisch gesetzte Anreize, wie Emissionsgrenzwerte, herrscht eine hohe Nachfrage nach effizienten, emissionsarmen Triebwerken. Die Beherrschung von Technologien, die Treibstoffeinsparungen ermöglichen, bedeutet daher einen direkten Wettbewerbsvorteil für einen Triebwerkshersteller.

Steigerungen des Gesamtwirkungsgrads von Flugzeugtriebwerken wurden in der Vergangenheit durch höhere Wirkungsgrade des Kerntriebwerks erreicht, die wiederum durch erhöhte Turbineneintrittstemperaturen und Druckverhältnisse ermöglicht wurden. Diese Fortschritte wurden zum Teil durch verbesserte Materialien für Turbinenschaufeln möglich. Seit den 1960er Jahren leistet die Kühlung von Turbinenschaufeln durch interne Kühlung und Filmkühlung einen erheblichen Beitrag zur Steigerung des thermischen Wirkungsgrads von Triebwerken. Aufgrund des hohen Wissensstands in diesen Forschungsbereichen sind weitere Fortschritte nur durch enormen Aufwand zu erreichen.

Neben dem thermischen Wirkungsgrad ist auch der Vortriebswirkungsgrad seit Einführung der ersten zivilen Strahltriebwerke kontinuierlich gestiegen. Turbofantriebwerke des 21. Jahrhunderts weisen Nebenstromverhältnisse von bis zu 10 auf und sind dadurch wesentlich effizienter als die ersten in der Luftfahrt eingesetzten Strahltriebwerke (Jane's Information Group (2005)).

Bei weiterer Erhöhung des Nebenstromverhältnisses gerät das Konzept des konventionellen Mantelstromtriebwerks an seine Grenzen. Höhere Nebenstromverhältnisse erfordern größere Fandurchmesser. Um transsonische Verluste an den Fanspitzen zu vermeiden und die mechanische Belastbarkeit des Fans zu gewährleisten, ist die Drehzahl der Niederdruckwelle begrenzt. Dadurch erfolgt der Enthalpieumsatz in der Turbine bei geringen Umfangsgeschwindigkeiten und geringer Stufenarbeit. Zum Antrieb des Fans können bis zu siebenstufige Niederdruckturbinen nötig sein. Höheres Gewicht, eine größere Anzahl an Bauteilen und höhere Kosten des Triebwerks sind die Folge hoher Stufenzahlen.

Durch ein Untersetzungsgetriebe zwischen Niederdruckturbine und Fan können beide Komponenten bei jeweils optimalen Drehzahlen betrieben werden. Bei einer weiteren Steigerung des Nebenstromverhältnisses können so höhere Vortriebswirkungsgrade bei einer geringeren Stufenanzahl der Niederdruckturbine realisiert werden. Das im Jahr 2016 eingeführte PW1000G, das unter anderem im Airbus A320neo eingesetzt wird, weist Nebenstromverhältnisse von bis zu 12,5 bei einer dreistufigen Niederdruckturbine auf. Dies führt zu Treibstoffeinsparungen von bis zu 16\% im Vergleich zu konventionellen Turbofantriebwerken (Pratt \& Whitney (2018)).

Dem Gewichtsvorteil durch eine geringere Anzahl Turbinenstufen steht das Gewicht des Getriebes gegenüber. Die übertragene Leistung in Triebwerken für sogenannte Single-Aisle-Flugzeuge liegt im Bereich von $20 \mathrm{MW}$, die Wellenleistung von Langstreckenflugzeugen kann über $50 \mathrm{MW}$ betragen. Die Übertragung der Leistung durch ein Getriebe bei derart hohen Leistungsdichten stellt eine enorme technische Herausforderung dar. Zusätzlich ist der verfügbare Bauraum innerhalb des Triebwerks begrenzt. 
Neben festigkeits- und schwingungsmechanischen Fragestellungen tritt mit der gesteigerten Leistungsdichte die Kühlung der Zahnräder und des gesamten Getriebes in den Vordergrund. Eine effiziente und effektive Kühlung mittels Öl ist erforderlich, um die thermische Belastung des Getriebes zu limitieren. Gleichzeitig wird aus Gründen der Wirtschaftlichkeit angestrebt, die Verluste im Getriebe zu minimieren, was einer Forderung nach möglichst geringen Ölvolumenströmen gleichkommt. Ein Optimum zwischen Schmierung, Kühlung und minimalen Verlusten kann nur dann erreicht werden, wenn der Wärmeübergang am Zahnrad durch Einspritzkühlung genau bekannt und verstanden ist. Dadurch leistet ein verbessertes Verständnis der Öleinspritzkühlung in Getrieben in mehrfacher Hinsicht einen Beitrag zur Senkung der Emissionen und Steigerung der Umweltverträglichkeit von Flugzeugtriebwerken.

In Untersuchungen zum Thermalhaushalt von Getrieben wird häufig die analytische Korrelation von DeWinter und Blok (1974) aufgegriffen, um den Wärmeübergang durch Einspritzschmierung zu bestimmen. Spätere experimentelle Untersuchungen haben jedoch gezeigt, dass die gemessenen Temperaturen und abgeschätzten Wärmeübergänge dadurch nicht immer zu erklären sind. Experimentelle Untersuchungen, in denen der Wärmeübergang an rotierenden Zahnrädern getrennt von Reibwärmeströmen und ortsaufgelöst bestimmt wird, existieren derzeit nicht.

Diese Lücke soll durch die vorliegende Arbeit geschlossen werden. Es wird eine experimentelle Methode und ihre Umsetzung in einem Prüfstand vorgestellt, mit der erstmalig der Wärmeübergang an einem einspritzgekühlten Zahnrad bestimmt werden kann.

Folgendes Vorgehen wurde gewählt, um einen Forschungsbeitrag zum Getriebefantriebwerk zu leisten: Zunächst wird in Kapitel 2 der derzeitige Stand der Wissenschaft zum Thermalhaushalt von hochbelasteten Getrieben geschildert. Darin wird deutlich, weshalb die Bestimmung des Öl-Zahnrad-Wärmeübergangs zur Entwicklung effizienter, hochbelasteter Getriebe für Flugtriebwerke unerlässlich ist. Aus dieser Erkenntnis wird die Zielsetzung dieser Arbeit abgeleitet. Da gegenwärtig keine Arbeiten bekannt sind, die das System der Einspritzkühlung klassifizieren, wird diese in Kapitel 3 zunächst beschrieben. Die zur Beschreibung des Einspritzvorgangs verwendeten Größen werden durch die Analyse des Systems abgeleitet. In Kapitel 4 wird die entwickelte Methode zur Bestimmung des Öl-Zahnrad-Wärmeübergangs vorgestellt und durch die Monte-Carlo-Methode hinsichtlich ihrer Messunsicherheit untersucht. Der zur experimentellen Umsetzung der Messmethode konzipierte Prüfstand wird in Kapitel 5 beschrieben. Die Diskussion der experimentellen Ergebnisse zum Öl-Zahnrad-Wärmeübergang, die durch diese Arbeit erstmalig quantitativ bestimmt werden, erfolgt in Kapitel 6. Die Einflüsse der Betriebsparameter werden diskutiert, anschließend wird eine Korrelation abgeleitet. In Kapitel 7 werden die Ergebnisse dieser Arbeit zusammengefasst. 


\section{Grundlagen und wissenschaftlicher Kenntnisstand}

Getriebe und Zahnräder sind ein elementarer Bestandteil der industrialisierten Welt. Dementsprechend umfangreich ist die Literatur zur Getriebetechnik. Die Arbeiten zum Thermalhaushalt von hochbelasteten Getrieben sind jedoch wesentlich weniger zahlreich. Besonders der ÖlZahnrad-Wärmeübergang durch Einspritzkühlung bedarf eines genaueren Verständnisses. Diese Notwendigkeit soll in diesem Kapitel herausgearbeitet werden.

Die Vorgänge und Phänomene, die sich in einem Getriebe abspielen, sind vielfältig, komplex und umspannen ein breites naturwissenschaftliches Feld. Für einfache Konstruktionen, wie sie die Mehrzahl der global eingesetzten Getriebe darstellen, ist die genaue Kenntnis dieser Vorgänge erlässlich und lässt sich durch Auslegung hin zu großen Sicherheitsvielfachen ersetzen. Mit steigenden Anforderungen an das Getriebe steigt die Notwendigkeit, die beteiligten Vorgänge genau zu verstehen. Wie für andere Maschinenelemente auch stellen die Luftfahrt und der Turbomaschinenbau im Besonderen extreme Anforderungen an Getriebe. Daraus entstehen erhöhte Anforderungen an das Detailverständnis der beteiligten physikalischen Vorgänge.

Die Herausforderung, ein Höchstleistungsgetriebe für ein Luftfahrttriebwerk zu entwickeln, zeichnet sich aus durch die Forderungen nach

- sehr hoher übertragener Leistung

- geringem Gewicht und geringem Bauraum

- hoher Zuverlässigkeit

- hohem Wirkungsgrad.

Die Erfüllung dieser Forderungen ist dadurch erschwert, dass sie stark gegensätzlich sind. Die Forderung nach hoher Leistungsdichte bei geringem Gewicht und Bauraum bedeutet unweigerlich hohe spezifische Wärmeströme durch Reibung und damit hohe lokale Materialtemperaturen, wie in Kapitel 2.1.1 erläutert wird. Über die Elastohydrodynamik, die in Kapitel 2.1.2 umrissen wird, lässt sich zeigen, dass hohe Materialtemperaturen für eine gegebene Getriebekonfiguration zu geringeren Schmierfilmdicken führen. Abnehmende Schmierfilmdicken haben Mischreibung im Eingriff zur Folge, bei der der Schmierfilm örtlich unterbrochen und die erwartete Lebensdauer der Zahnräder herabgesetzt wird. Dies steht der Forderung nach hoher Zuverlässigkeit entgegen. Die Begegnung dieses Problems durch den verstärkten Einsatz von Öl, dessen Hauptfunktion die Kühlung der Zahnräder ist, bringt jedoch Einbußen im Wirkungsgrad mit sich, da Ventilations- und Planschverluste in Getrieben mit der verwendeten Ölmenge ansteigen. Der derzeitige wissenschaftliche Kenntnisstand hält keine Hinweise darauf bereit, wie die Kühlwirkung der eingesetzten Ölmenge maximiert werden kann. Die in Abschnitt 2.2 beschriebenen Ansätze zur Thermalmodellierung erlauben zwar die Modellierung der sich einstellenden Zahnflankentemperaturen. Die benötigten Randbedingungen zum Zahnflankenwärmeübergang bei Einspritzkühlung sind jedoch nur als grobe Abschätzung bekannt, wie in Abschnitt 2.3 verdeutlicht wird. Die Bestimmung der Randbedingungen aus allgemeineren Arbeiten zur Prallkühlung 
ist nur überschlägig möglich, wie in Abschnitt 2.4 dargelegt wird. Wird also ein Optimum zwischen den gegensätzlichen Anforderungen gesucht, müssen zunächst die gekoppelten und komplexen Vorgänge im Getriebe verstanden werden. Erst dann können die oben genannten Forderungen bestmöglich erfüllt werden.

\subsection{Getriebemechanik}

Das Zahnrad als Element zur Übertragung von Leistung und zur Übersetzung von Drehzahl und Drehmoment gilt als eines der ältesten Maschinenelemente der Welt. Die erste eingehende mathematische Beschreibung von Zahnformen geht auf das Jahr 1694 und Philippe de la Hire zurück, der darin die epizykloide Zahnform beschreibt. Die heute gebräuchlichste Form, die Evolventenverzahnung (siehe Abbildung 2.1), wurde von Leonard Euler 1760 erstmals beschrieben (Klocke und Brecher (2017)). Ihr Vorteil besteht in der gleichmäßigen und damit weitgehend vibrationsfreien Übertragung der Drehbewegung. Diese Eigenschaft ist für den verschleißund geräuscharmen Betrieb von großer Bedeutung. Sie lässt sich mathematisch als Forderung

nach $\frac{\omega_{1}}{\omega_{2}} \stackrel{!}{=}$ const. für die Rotationsgeschwindigkeit des treibenden und angetriebenen Zahnrads, $\omega_{1}$ und $\omega_{2}$, zu jedem Zeitpunkt während des Eingriffs ausdrücken. Sie ist dann erfüllt, wenn die Berührnormale des Eingriffspunkts den Wälzpunkt schneidet. Dieser Zusammenhang ist als Verzahnungsgesetz bekannt, das außer bei der erwähnten Evolventenverzahnung auch bei Zykloiden-, Triebstock-, Kreisbogen- und Wildhaber-Novikov-Verzahnungen erfüllt ist (Klocke und Brecher (2017)). Aufgrund der herausragenden Bedeutung der Evolventenverzahnung im Luftfahrtumfeld und für den Kontext des Höchstleistungsgetriebes soll diese in dieser Arbeit ausschließlich betrachtet werden.

\subsubsection{Reibung und Verluste}

In Abbildung 2.1 sind zwei im Eingriff befindliche Zahnräder mit Evolventenverzahnung schematisch dargestellt. Der Punkt A stellt dabei den Beginn, Punkt E das Ende eines Zahnradeingriffs dar. Die Summe aller Berührpunkte zwischen den Punkten A und E wird Eingriffstrecke genannt. Bei der dargestellten Evolventenverzahnung ist diese Strecke eine Gerade, die sogenannte Eingriffsgerade $\overline{A E}$. Im Berührpunkt des treibenden und des angetriebenen Zahnrads liegt dabei, mit Ausnahme des Wälzpunktes, stets eine Relativgeschwindigkeit der Oberflächen vor. Dies wird durch die Darstellung der Gleitgeschwindigkeiten des treibenden und des getriebenen Zahnrads, $v_{\mathrm{g} 1}$ und $v_{\mathrm{g} 2}$, deutlich. Am Wälzpunkt $C$ erfahren sie einen Vorzeichenwechsel. Der Verlauf der Geschwindigkeiten ist linear über die Eingriffstrecke. Diese Gleitbewegung ist ursächlich für die entstehende Reibwärme im Getriebe, die einen wesentlichen Anteil an den Gesamtverlusten hat.

Die Verlustleistung kann bei gering belasteten Getrieben allein durch Wärmeleitung über die Zahnradkörper abgeführt werden, wodurch die Materialtemperaturen in einem unkritischen Bereich bleiben. Mit steigender Last können jedoch kritische Temperaturwerte erreicht werden, die zu einer Lebensdauerreduzierung oder gar zum unmittelbaren Versagen des Getriebes führen. Um für erhöhte Lasten Dauerfestigkeit sicherzustellen, werden Getriebe daher mit einem 


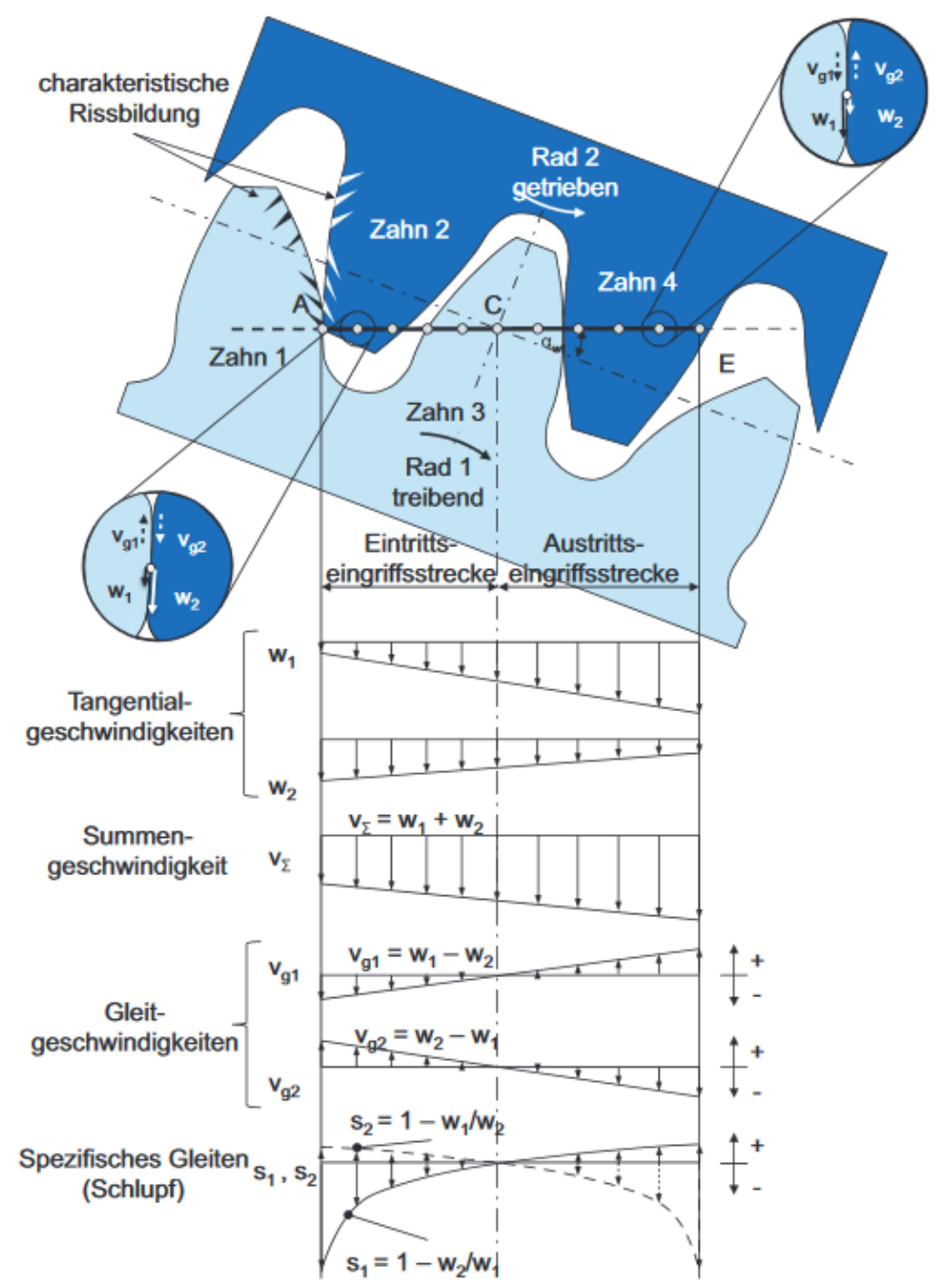

Abbildung 2.1: Schematische Darstellung des Eingriffs zweier evolventischer Zahnräder und der auftretenden Geschwindigkeiten nach Klocke und Brecher (2017)

Schmiermittel versehen. Zum einen dient dieses dazu, den Reibwert $\mu$ und damit die Verlustbzw. Reibleistung $P_{\mathrm{Vz}}$ herabzusetzen. Zum anderen kann durch das Schmiermittel auch Wärme abgeführt werden. In einfachen Konstruktionen gibt das Schmiermittel die aufgenommene Wärme an das Gehäuse ab (Sumpfschmierung), in aufwendigeren Konstruktionen können dafür Ölkühler eingesetzt werden. Mit steigender Leistungsdichte tritt der Aspekt der Kühlung durch das Öl zunehmend in den Vordergrund. Das gilt auch für andere tribologische Maschinenelemente wie beispielsweise Wälzlager, deren Schmierung und Kühlung durch Öl am Institut für Thermische Strömungsmaschinen seit mehreren Jahrzehnten Gegenstand intensiver Forschung ist (Glahn et al. (1997), Gorse et al. (2003)).

Die Kühlung und Schmierung von Wälzlagern in Triebwerken erfolgt, wie im vorliegenden Fall, häufig durch Einspritzkühlung (Krug (2018)). Zur Kühlung der Wälzlager ist eine ausreichende Zufuhr von Öl sicherzustellen. Das Öl interagiert, nachdem es die Einspritzdüse verlässt, mit der von der Welle mitbewegten Luft in der Lagerkammer und kann dadurch zerstäubt werden. 
Dadurch erreicht nur ein Anteil des eingespritzten Ölvolumenstroms das Lager, der durch den Auffangwirkungsgrad beschrieben wird. (Krug et al. (2014)) zeigen, dass die Wellendrehzahl und der eingespritzte Ölvolumenstrom entscheidend für den Auffangwirkungsgrad sind. Das eingespritzte Öl nimmt anschließend an den Strömungsvorgängen der Lagerkammer teil und beeinflusst diese. Kurz und Bauer (2014) identifizieren zwei charakteristische Strömungsregime, die vor allem vom Lagerkammerdurchmesser und dem Ölvolumenstrom durch die Lagerkammer abhängen. Die Strömung in der Lagerkammer, die sich durch die Geometrie und die Betriebsbedingungen einstellt, ist entscheidend für den Wärmeübergang an den Lagerkammerwänden. Sowohl Glahn (1995) als auch Kurz (2018) charakterisieren diesen Zusammenhang experimentell. Die Kenntnis der komplexen Vorgänge dient im Kontext der Lagerkammer zur Steigerung der umsetzbaren Leistungsdichte.

Durch den Einsatz von Öl als Schmier- und Kühlmittel können auch in einem Getriebe höhere Leistungsdichten realisiert werden. Gleichzeitig treten dadurch zusätzliche Verluste auf, da das Öl zwangsläufig an den komplexen Strömungsvorgängen beteiligt ist. Eine häufig getroffene Aufteilung der Getriebeverluste ist die von Niemann und Winter (2003):

$$
P_{\mathrm{V}}=\overbrace{P_{\mathrm{Vz}}+P_{\mathrm{Vz} 0}}^{\text {Zahnräder }}+\overbrace{P_{\mathrm{VB}}+P_{\mathrm{VB} 0}}^{\text {Lager }}+P_{\mathrm{VD}}+P_{\mathrm{VX}}
$$

Darin sind $P_{\mathrm{Vz}}$ und $P_{\mathrm{Vz} 0}$ die Verzahnungsverluste, $P_{\mathrm{VB}}$ und $P_{\mathrm{VB} 0}$ die Lagerverluste, $P_{\mathrm{VD}}$ die Dichtungsverluste und $P_{\mathrm{VX}}$ sonstige Verluste. In Gleichung 2.1 kennzeichnet der Index 0 diejenigen Verluste, die lastunabhängig sind. Diese lastunabhängigen Verluste stellen sich unabhängig von der übertragenen Leistung bzw. dem übertragenen Moment ein und beinhalten Plansch-, Ventilations- und Verdrängungsverluste. Während für langsam drehende Getriebe und hohe Drehmomente die lastabhängigen Verluste dominieren, überwiegen bei Hochdrehzahlanwendungen die lastunabhängigen Verluste (Diab et al. (2006a)).

Bei der Sumpfschmierung, die auch Tauchschmierung genannt wird, tauchen die Zahnräder in ein Ölbad ein und werden allein durch ihre Eigenbewegung mit Öl versorgt und gekühlt. Die Sumpfschmierung stellt die einfachste konstruktive Methode der Ölversorgung dar. Diesem Vorteil stehen Verluste entgegen, die durch das vergleichsweise hohe Ölvolumen entstehen, das an den Strömungsvorgängen im Getriebe beteiligt ist. Durch die Reduzierung der Füllstandshöhe des Öls können diese Verluste gesenkt werden (Seetharaman et al. (2009), Michaelis et al. (2011)). Bei zu geringen Füllstandshöhen ist jedoch sowohl mit mangelnder Benetzung der Zahnräder mit Öl als auch mit unzureichender Kühlung zu rechnen, was zur Beschädigung der Zahnräder führen kann (Höhn et al. (2008)). Schober (1983b) nennt eine Grenze der Umfangsgeschwindigkeit von Zahnrädern von etwa $60 \mathrm{~m} / \mathrm{s}$, bis zu der Sumpfschmierung sinnvoll eingesetzt werden kann. Bei Planetengetrieben, wie sie für den Einsatz in Höchstleistungsgetrieben für Getriebefantriebwerke in Frage kommen, scheidet der Einsatz von Sumpfschmierung aus.

Durch Einspritzen des Öls können Schmierung und Kühlung der Zahnräder bei höheren Umfangsgeschwindigkeiten ermöglicht werden, als es mit Sumpfschmierung möglich ist. Dem größeren konstruktiven Aufwand stehen dabei geringere Verluste gegenüber (Schober (1983a), Andersson et al. (2017)). In einspritzgeschmierten Getrieben sind die lastunabhängigen Verluste 
vor allem von der Drehzahl, der Geometrie der Zahnräder und der unmittelbaren Umgebung sowie der Dichte des umgebenden Fluids abhängig (Eastwick und Johnson (2008)).

Der Zusammenhang zwischen der Dichte des Fluids im Getriebe und lastunabhängigem Verlust bedeutet, dass eine zunehmende Konzentration von Öl in Form von feinen Tropfen eine Erhöhung der Verluste mit sich bringt. Erhöhte Konzentrationen sind dann zu erwarten, wenn mehr Öl am Schmier- und Kühlprozess teilnimmt. Zusätzlich hat der Impulsaustausch zwischen eingespritztem Öl und Zahnrad unmittelbar Verluste zur Folge, die mit zunehmender Ölmenge steigen (Massini et al. (2017)). Eine geringere Menge eingespritzten Öls führt daher zu geringeren Verlusten. Darüber hinaus können so die benötigten Pumpen und Übertragungssysteme kleiner dimensioniert werden. Dies führt zu einem geringeren benötigten Bauraum der Systeme und zu Gewichtseinsparungen.

Für fortschrittliche, hochbelastete Getriebe mit hohen Drehzahlen sind Wirkungsgrade zwischen 98\% und 99\% erreichbar (Eastwick und Johnson (2008)). Im Fall des Hilfsgerätegetriebes von Luftfahrttriebwerken können die Strömungsverluste dabei mehr als 50\% des Gesamtverlustes ausmachen (Arisawa et al. (2014)).

Für den Thermalhaushalt des Zahnrads, der in Abschnitt 2.2 eingehend betrachtet wird, ist besonders die Verlustleistung im Eingriff, die durch $P_{\mathrm{Vz}}$ erfasst wird, von Bedeutung. Diese wird unmittelbar an den belasteten Zahnflanken und somit auf kleiner Fläche freigesetzt, was zu starker Erwärmung der Zahnflanken führt. Die lastunabhängigen Verluste entstehen durch Dissipationsvorgänge, die sich im gesamten vom Getriebe eingeschlossenen Volumen abspielen. Sie führen zu einer Erwärmung des Kühlmittels, sind jedoch für die Zahnradtemperaturen nur von mittelbarer Bedeutung.

Die durch die Gleitbewegung entstehende Verlustleistung $P_{\mathrm{Vz}}$ kann ausgedrückt werden als:

$$
P_{\mathrm{Vz}}=F_{\mathrm{R}} v_{\mathrm{g}}=\left(1 / p_{\mathrm{e}}\right) \int_{g} F_{\mathrm{n}} \mu v_{\mathrm{g}} \mathrm{d} g
$$

mit der Reibkraft $F_{\mathrm{R}}$, der Gleitgeschwindigkeit $v_{\mathrm{g}}$, der Eingriffsteilung $p_{\mathrm{e}}$, der Normalkraft $F_{\mathrm{n}}$ und der Eingriffstrecke $g$. Der Reibungsbeiwert $\mu$ ist eine örtliche Größe, die unter anderem von der Materialpaarung und der Art der Schmierung abhängig ist. In Gleichung 2.2 ist nur der Reibungsbeiwert $\mu$ nicht von der Konfiguration des Getriebes abhängig. Neben ihrer Bedeutung für den Thermalhaushalt des Getriebes ist die Verlustleistung $P_{\mathrm{Vz}}$ auch für die Wirkungsgradbetrachtung in Getrieben wichtig.

Die Bestimmung des Reibungsbeiwerts $\mu$ kann experimentell erfolgen. Mittlere Werte für $\mu$ können jedoch auch durch empirische Näherungsformeln wie der von Winter und Michaelis (1984) bestimmt werden:

$$
\mu_{\mathrm{m}}=0,045\left(\frac{K_{\mathrm{A}} F_{\mathrm{n}} / b}{v_{\Sigma \mathrm{m}} \rho_{\mathrm{m}}}\right)^{0,2} \eta_{0}^{-0,05} X_{\mathrm{R}} \leq 0,2 .
$$

Darin ist $K_{\mathrm{A}}$ ein Anwendungsfaktor, der von der Art der Belastung abhängt, $b$ die Breite des Zahnrads, $v_{\Sigma \mathrm{m}}$ die mittlere Summengeschwindigkeit und $\rho_{\mathrm{m}}$ der mittlere Ersatzkrümmungsradius. Die Summengeschwindigkeit ist die Summe der Tangentialgeschwindigkeiten (siehe 
Abbildung 2.1) und beeinflusst den Aufbau des Schmierfilms maßgeblich. $\eta_{0}$ ist die Viskosität des Öls bei Massentemperatur, also der Temperatur der Zahnradoberfläche. $X_{\mathrm{R}}$ ist ein Faktor für die Rauigkeit, mit

$$
X_{\mathrm{R}}=3,8\left(R_{\mathrm{a}} / d_{\mathrm{w}}\right)^{\frac{1}{4}} .
$$

Zur Berechnung des instantanen Reibungsbeiwerts an einem beliebigen Punkt der Eingriffsgerade werden in der Literatur häufig die Korrelation von Kelley und Lemanski (1967)

$$
\mu=0,0099 \frac{1}{1-R_{\mathrm{a}} / 45} \log _{10}\left[\frac{50,8 F_{\mathrm{n}} / b}{\eta_{0} v_{g} v_{\Sigma}^{2}\left(R_{1}+R_{2}\right)^{2}}\right]
$$

oder die Korrelation von Benedict und Kelley (1961)

$$
\mu=0,0127 \cdot \log _{10}\left(\frac{3,17 \cdot 10^{8} F_{\mathrm{n}} / b}{\eta_{0} v_{g} v_{\Sigma}^{2}}\right)
$$

verwendet. Gleichungen 2.5 und 2.6 liefern Reibungsbeiwerte, die im Allgemeinen mit den Werten experimenteller Untersuchungen übereinstimmen. Allen genannten Bestimmungsansätzen für den Reibwert ist gemein, dass der Reibwert bei höheren Oberflächen- und Gleitgeschwindigkeiten abnimmt, während höhere bezogene Normalkräfte zu höheren Reibwerten führen.

Diab et al. (2006b) zeigen, dass die genannten Ansätze den Reibwert aufgrund der geringen relativen Gleitgeschwindigkeiten an Zahnrädern tendenziell überschätzen. Effekte wie die Anisotropie und die Orientierung der Oberflächenrauigkeit gefertigter Zahnräder führen zusätzlich zu Abweichungen des tatsächlichen Reibwerts im Vergleich zu Korrelationen.

Wesentlich genauere Vorhersagen des Reibwerts könnten getroffen werden, indem die elastohydrodynamischen Vorgänge vollständig modelliert werden. Britton et al. (2000) zeigen anhand derartiger Berechnungen und experimenteller Untersuchungen unter identischen Bedingungen sehr geringe Abweichungen der Werte.

Festzuhalten ist, dass der Reibwert $\mu$ von der Konfiguration des Getriebes, dem Lastzustand und dem thermischen Zustand der Zahnräder abhängt. Hohe Normalkräfte im Eingriff können dabei den Reibwert erhöhen, jedoch ist die Abhängigkeit nach Gleichung 2.3 mit

$$
\mu_{\mathrm{m}} \propto F_{\mathrm{n}}^{0,2}
$$

oder nach Gleichungen 2.5 und 2.6 mit

$$
\mu \propto \log _{10}\left(F_{\mathrm{n}}\right)
$$

schwach. Wird die Leistung in einem Getriebe auf kleinerem Bauraum umgesetzt, ist nur mit geringen Steigerungen des Reibwerts und somit der lastabhängigen Verluste zu rechnen. Die Fläche, auf der die Reibleistung in Form von Wärme freigesetzt wird, sinkt jedoch. Dadurch ist in Höchstleistungsgetrieben, wie sie im Rahmen dieser Arbeit betrachtet werden, mit hohen Wärmestromdichten durch Reibung zu rechnen. Der Kühlung der Zahnräder bei hohen Umfangsgeschwindigkeiten kommt somit besondere Bedeutung zu. 


\subsubsection{Tribologie}

Schmierstoffe wurden bereits eingesetzt, um Verschleiß und Verluste in Reibkontakten zu reduzieren, bevor die zu Grunde liegenden Mechanismen der Schmierfilmbildung und der Kontaktmechanik bekannt oder verstanden waren (Dowson et al. (1977)). Die erstmalige Beschreibung dieser tribologischen Mechanismen erfolgt durch Reynolds (1886). Reynolds schlägt einen mathematischen Lösungsansatz für die von Tower (1883) beobachteten, äußerst geringen Reibungen zwischen vollständig in Öl eingetauchten Oberflächen vor. Dieser beruht auf der Annahme eines durchgehenden Schmierfilms zwischen den Oberflächen, einem wesentlichen Gedanken der Tribologie. Aufbauend darauf vereinfacht Reynolds die hydrodynamischen Grundgleichungen, um zu einem Ausdruck für die sich einstellende Schmierfilmdicke zu gelangen. Die Auswertung dieses Ausdrucks ergibt für die real umgesetzten hohen Normallasten stets sehr kleine Schmierfilmdicken, die wesentlich kleiner als die herstellbaren Oberflächenrauigkeiten sind. Die klassische Hydrodynamik scheitert demnach bei der Erklärung eines Schmierfilms für Zahnräder unter den technisch bereits umgesetzten Lasten und Kontaktdrücken.

Erst die Berücksichtigung zweier weiterer wesentlicher Aspekte liefert plausible Schmierfilmdicken:

- Die Viskosität typischer Schmieröle steigt unter hohem Druck erheblich an. Der Faktor liegt üblicherweise bei $10^{4}$. Dies ist als Piezoviskoser Effekt bekannt.

- Die Kontaktflächen der Zahnräder verformen sich im Eingriff elastisch.

Die Betrachtung des Schmierfilms unter Berücksichtigung der beiden genannten Effekte wird in der Literatur als Elastohydrodynamik (EHD) bezeichnet (Olver (2002)) und wurde in den 1950er und -60er Jahren etabliert (Grubin und Vinogradova (1949), Barwell (1958), Dowson et al. (1977)). Erst durch die Theorie der Elastohydrodynamik lassen sich Filmdicken erklären, die mit experimentell ermittelten Werten in Einklang gebracht werden können. Die Erkenntnisse der 1960er Jahre, die von Dowson und Higginson zusammengefasst werden (Dowson et al. (1977)), werden im Bereich der Getriebemechanik aufgegriffen und erweitert, um auch Schmierfilme zwischen gleitenden Oberflächen zu beschreiben. Eine solche Gleitbewegung liegt bei der Evolventenverzahnung zwangsläufig vor.

In Abbildung 2.2 ist der qualitative Druckverlauf des Schmierfilms im Eingriff zweier Wälzkörper sowohl für die Hertz'sche Theorie als auch für die EHD-Theorie dargestellt. Über die Hertz'sche Theorie kann die Druckverteilung sich berührender Festkörper ohne den Einfluss eines Schmierfilms bestimmt werden (Hertz (1881)). In weiten Bereichen stimmt die Druckverteilung beider Theorien überein. Ein wichtiges Merkmal des Verlaufs der Schmierfilmdicke, die durch die EHD-Theorie bestimmt wird, ist die Einschnürung am Eingriffsauslass. An der Stelle der Einschnürung ist ein Druckmaximum zu erkennen, das charakteristisch für EHDSchmierfilme ist und Petrusevich'sche Spitze genannt wird. Diese Einschnürung wird durch die Theorie vollständig erklärt (Dowson et al. (1977)) und ist experimentell bestätigt. Ihr kommt besondere Bedeutung zu, da sie die Stelle dünnster Schmierfilmdicke darstellt und somit für Verschleißbetrachtungen essentiell ist. 


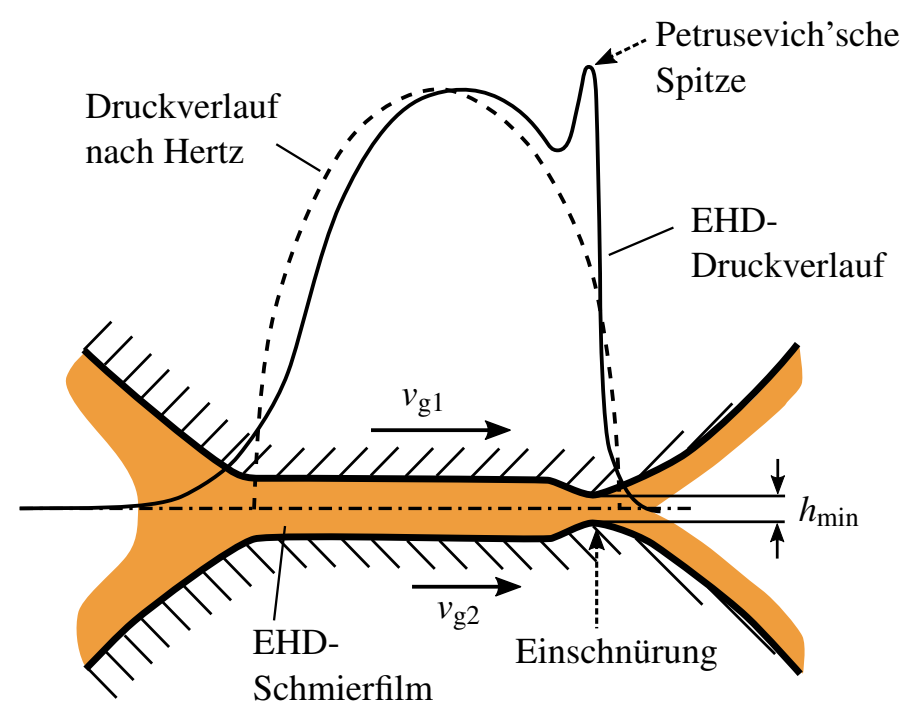

Abbildung 2.2: Druckverteilung im Schmierspalt nach Hertzscher und elastohydrodynamischer Theorie und charakteristischer EHD-Schmierfilm nach Theißen (2003)

Die exakte Vorhersage von Schmierfilmdicken für unterschiedliche tribologische Systeme wie Gleitlager oder Zahnräder ist aufgrund der Vielzahl beteiligter Vorgänge schwierig. Die Deformation der Festkörper und die Fluidmechanik des Schmierfilms sind auch für einfachste Betrachtungen zu berücksichtigen, um korrekte Ergebnisse zu erhalten. Je nach Anwendungsfall können weitere physikalische Vorgänge, wie beispielsweise Wärmetransportvorgänge (Pinkus (1990)) in oder aus dem Schmierfilm, einen Einfluss auf die Schmierfilmdicke haben. Betrachtung und Bewertung des Einzelfalls sind umfangreich und sollen im Rahmen dieser Arbeit nicht angestellt werden.

Dowson et al. (1977) stellen jedoch fest, dass die Betrachtung der Schmierfilmdicke anhand dreier dimensionsloser Kennzahlen $G, W$ und $U$ für alle bekannten theoretischen Lösungen durch eine Näherungsgleichung bereits gute Ergebnisse liefert. Dazu wird der Materialparameter

$$
G=\alpha E^{\prime}
$$

definiert. Dieser ist über den Druck-Viskositätskoeffizienten $\alpha$ vom Schmiermittel und den Ausdruck $E^{\prime}=\frac{E}{1-v^{2}}$ vom Wälzkörpermaterial abhängig. Über $\alpha$ wird der eingangs erwähnte, starke Viskositätsanstieg der meisten Öle für sehr hohe Drücke berücksichtigt. $E^{\prime}$ ist eine Funktion des E-Moduls $E$ und der Querkontraktionszahl $v$. Für eine definierte Kombination aus Schmiermittel und Zahnradmaterial ist $G$ eine Konstante.

Der Lastparameter

$$
W=\frac{w}{E^{\prime} R}
$$

ist abhängig von der längenbezogenen Normalkraft $w=\frac{F_{\mathrm{n}}}{l} . F_{\mathrm{n}}$ ist die Kraft, die normal zur Berührfläche auf den tribologischen Kontakt bzw. den Eingriff wirkt. $l$ ist die Eingriffslänge, über die die Kraft $F_{\mathrm{n}}$ übertragen wird. Für einen gegebenen Lastfall ist auch $W$ eine Konstante. 
Der Geschwindigkeitsparameter

$$
U=\frac{\eta_{0} u}{E^{\prime} R}
$$

ist abhängig vom äquivalenten Wälzkörperradius $R$, der Oberflächengeschwindigkeit $u$ sowie $\eta_{0}$. Die Viskosität $\eta_{0}$ ist die Viskosität des Schmiermittels bei der Materialtemperatur zu Beginn des Eingriffs. Bei gegebenen Betriebszuständen und für ein ausgeführtes Getriebe ist nur $\eta_{0}$ eine Variable. Die minimale Schmierfilmdicke $h_{\text {min }}$ kann aus den obenstehenden dimensionslosen Kennzahlen nach Dowson et al. (1977) durch den Ausdruck

$$
h_{\min }=1,6 R \frac{G^{0,6} U^{0,7}}{W^{0,13}}
$$

bestimmt werden. Durch Gleichung 2.12 ist die für die Auslegung von Verzahnungen und Wälzkörpereingriffen im Allgemeinen wichtige minimale Schmierfilmdicke, $h_{\min }$, durch eine sehr einfache mathematische Beschreibung zugänglich. Sie ist im Bereich der elastohydrodynamischen Schmierung gültig, die für Bereiche von $U$ und $W$ vorliegt, die nahezu alle real auftretende Anwendungsfälle abdecken. Die Kennzahlen der im Rahmen dieser Arbeit betrachteten, hochbelasteten Getriebe liegen stets im Gültigkeitsbereich von Gleichung 2.12. Durch die Abhängigkeit von $h_{\min }$ von $W$ mit einem Exponenten von -0,13 ist die minimale Schmierfilmdicke nur äußerst schwach von der Zahnradlast abhängig.

Die Anwendbarkeit von Gleichung 2.12 ist durch zahlreiche Veröffentlichungen eindeutig belegt. Auch in der Normungsliteratur wird sie als Auslegungskriterium zur Sicherheit gegen Warmfressen genutzt. Die internationale Norm zur Auslegung von hochbelasteten Getrieben mit Sicherheit gegen Graufleckenbildung, ISO TR 15144, wurde im Jahr 2014 veröffentlicht und verwendet Gleichung 2.12 als Auslegungskriterium (ISO (2014)).

Für ein gegebenes Getriebe mit gegebener Lastanforderung und Geometrie sind der Lastparameter $W$ und der Materialparameter $G$ konstant. Der Geschwindigkeitsparameter $U$ ist, bei konstantem $u$, ausschließlich eine Funktion von $\eta_{0}$, der Viskosität des Schmiermittels bei Umgebungsdruck. Für die Schmierfilmdicke $h_{\text {min }}$ gilt daher:

$$
h_{\min } \propto \eta_{0}^{0,7}\left(T_{0}\right)
$$

Somit ist die temperaturabhängige Viskosität $\eta_{0}\left(T_{0}\right)$ des Öls unmittelbar vor dem Eingriff der Zahnräder entscheidend für die Dicke des Schmierfilms. Dabei ist $T_{0}$ nicht etwa die Einspritztemperatur des Öls oder die Temperatur im Ölsumpf, sondern die Temperatur der Zahnräder vor dem Eingriff. In der englischsprachigen Literatur wird für $T_{0}$ üblicherweise der Begriff bulk temperature verwendet.

Zur Bestimmung der Schmierfilmdicke sind durch die genannten Zusammenhänge die Viskosität des Schmiermittels und ihr Temperaturverhalten entscheidend. Typische Schmieröle im Luftfahrtbereich besitzen einen Viskositäts-Temperaturverlauf, der durch die Arrhenius-AndradeBeziehung angenähert werden kann. Grundsätzlich sinkt die Viskosität mit steigender Temperatur. Dies gilt auch für das häufig verwendete Mobil Jet Oil II, dessen Temperaturverlauf von Glahn 
(1995) beschrieben wird. Die EHD-Schmierfilmdicke für Jet Oil II ist in Abbildung 2.3, bezogen auf die Schmierfilmdicke bei $100^{\circ} \mathrm{C}$ und für einen generischen Lastfall, dargestellt. Eine Erhöhung der Temperatur von $100^{\circ} \mathrm{C}$ auf $148^{\circ} \mathrm{C}$ führt zu einer Halbierung der Schmierfilmdicke.

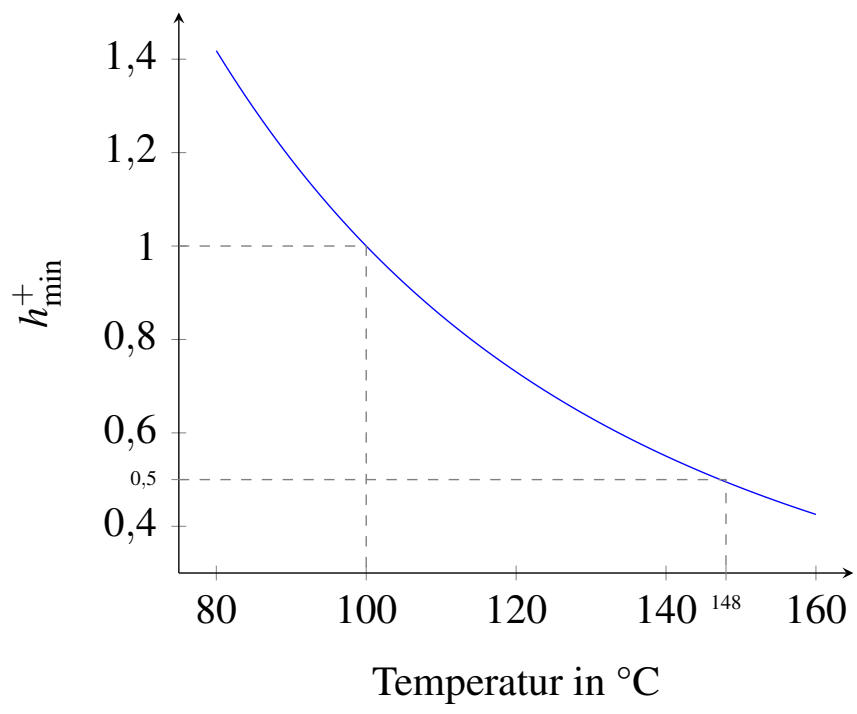

Abbildung 2.3: Einfluss der Oberflächentemperatur vor dem Eingriff auf die minimale relative Schmierfilmdicke

Die Schmierfilmdicke ist entscheidend für mehrere Schadensmechanismen, die die Leistungsdichte und die Lebensdauer eines Getriebes begrenzen. In Abbildung 2.4 sind die möglichen Zustände des dünner werdenden Schmierfilms dargestellt. Bei ausreichend großem $h_{\min }$ ist stets ein durchgängiger Schmierfilm zwischen den Zahnflanken vorhanden, die Gleitbewegung führt lediglich zu einer Scherung des Fluids im Schmierspalt unter Reibwärmeentwicklung (engl.: Full-film EHD, Flüssigkeitsreibung). Bei reduzierter Schmierfilmdicke nähern sich lokale Rauigkeitsspitzen an, es kommt zunächst zur Ausbildung lokaler, kleinstskaliger Schmierfilme (engl.: Micro-EHD) und anschließend zu örtlich begrenzten Unterbrechungen des Schmierfilms (engl.: Mixed lubrication, Mischreibung). Ab einer gewissen Konzentration solcher Unterbrechungen wird diese Mischreibung kritisch. Durch die Festkörperreibung der beteiligten Flanken kommt es zu kurzzeitigen Temperaturspitzen im Metall. Im Falle des sogenannten Fressens erreicht das kurzzeitige Temperaturniveau den Schmelzpunkt des Metalls, es kommt zum Verschweißen der Berührflächen und unmittelbar darauf folgendem Aufreißen der Verbindung (Niemann und Winter (2003)). Ist der Schmierfilm vollständig unterbrochen, spielt das Schmiermittel nur noch in kleinstskaligen Vorgängen eine Rolle (engl.: Boundary lubrication).

Die Oberflächenrauigkeit in Verbindung mit der minimalen Schmierfilmdicke ist entscheidend für den Zustand im Schmierspalt. Zur Bewertung der Sicherheit gegen Warmfressen wird die spezifische Schmierfilmdicke

$$
\lambda_{\mathrm{GF}}=\frac{h_{\mathrm{min}}}{R_{\mathrm{a}}}
$$

unter Verwendung der mittleren Rautiefe

$$
R_{\mathrm{a}}=\frac{R_{\mathrm{a} 1}+R_{\mathrm{a} 2}}{2}
$$




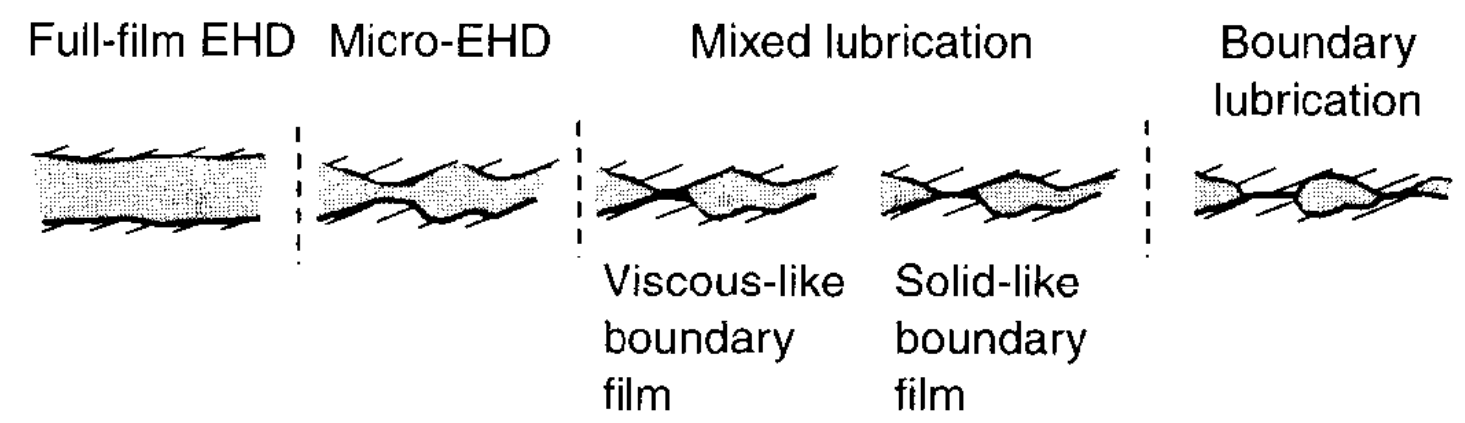

Abbildung 2.4: Schmierfilmzustände im Zahnradeingriff nach Spikes (1997)

mit den Rauigkeiten der beiden Flanken, $R_{\mathrm{a} 1}$ und $R_{\mathrm{a} 2}$, verwendet. $\lambda_{\mathrm{GF}}$ wird typischerweise in die in Tabelle 2.1 aufgeführten Bereiche aufgeteilt.

Tabelle 2.1: Bewertung der spezifischen Schmierfilmdicke $\lambda_{\mathrm{GF}}$

\begin{tabular}{cc}
\hline$\lambda_{\mathrm{GF}}$ & Schmierfilmzustand \\
$>2$ & Überwiegend hydrodynamische Schmierung \\
$0,7-2$ & Grenzschmierung \\
$<0,7$ & Festkörperreibung \\
\hline
\end{tabular}

Der Übergang von reiner Flüssigkeitsreibung zur Festkörperreibung über den Bereich der Mischreibung verläuft kontinuierlich und hängt von einer Vielzahl weiterer Faktoren ab. So spielt auch die Gestalt der beteiligten Rauigkeiten eine Rolle (Guegan et al. (2016)). Es wird beobachtet, dass bereits ab einem Wert $\lambda_{\mathrm{GF}}$ von 2-3 erste Rauigkeitsspitzen in Berührung kommen und bis zu einem Wert von 1 der Großteil der Last vom Schmierfilm getragen wird (Spikes (1997)).

Krantz (2014) untersucht den Einfluss der spezifischen Schmierfilmdicke auf die Lebensdauer der beteiligten Zahnräder experimentell. Dazu werden Zahnradpaare in einem Prüfstand unter kontinuierlicher Leistungsübertragung so lange betrieben, bis es zu Schäden an den Flanken unter Entstehung von Vibrationen und Ausfall der Verzahnung kommt. Durch unterschiedliche Kombinationen aus Schmiermittel, Rauigkeit des Zahnrads und Zahnradmaterial werden unterschiedliche spezifische Schmierfilmdicken erzeugt. Die Verteilung der durchlaufenen Zyklen bis zum Versagen in Abhängigkeit der spezifischen Schmierfilmdicke ist in Abbildung 2.5 dargestellt. Während die Lebensdauer bei $\lambda_{\mathrm{GF}}>2$ unabhängig von der Schmierfilmdicke ist, nimmt sie bei Unterschreiten eines Wertes von etwa 2 stark ab. Bei Schmierfilmen, die mit $\lambda_{\mathrm{GF}}<1$ dünner als die mittlere Rautiefe der Zahnräder sind, werden deutlich reduzierte minimale Werte für die Lebensdauer gemessen.

Grundsätzlich wird das Auftreten von Festkörperreibung bei abnehmenden Schmierfilmdicken mit erhöhter Gefahr des Verschleißes in Verbindung gebracht. Bei additivierten Schmierstoffen kann bei erhöhten Temperaturen jedoch die chemische Aktivität des Additivs erhöht sein. Die dadurch zunehmende Schutzwirkung kann den negativen Einfluss abnehmender Viskosität und zunehmenden Festkörperkontakts hinsichtlich des Verschleißes unter Umständen kompensieren 


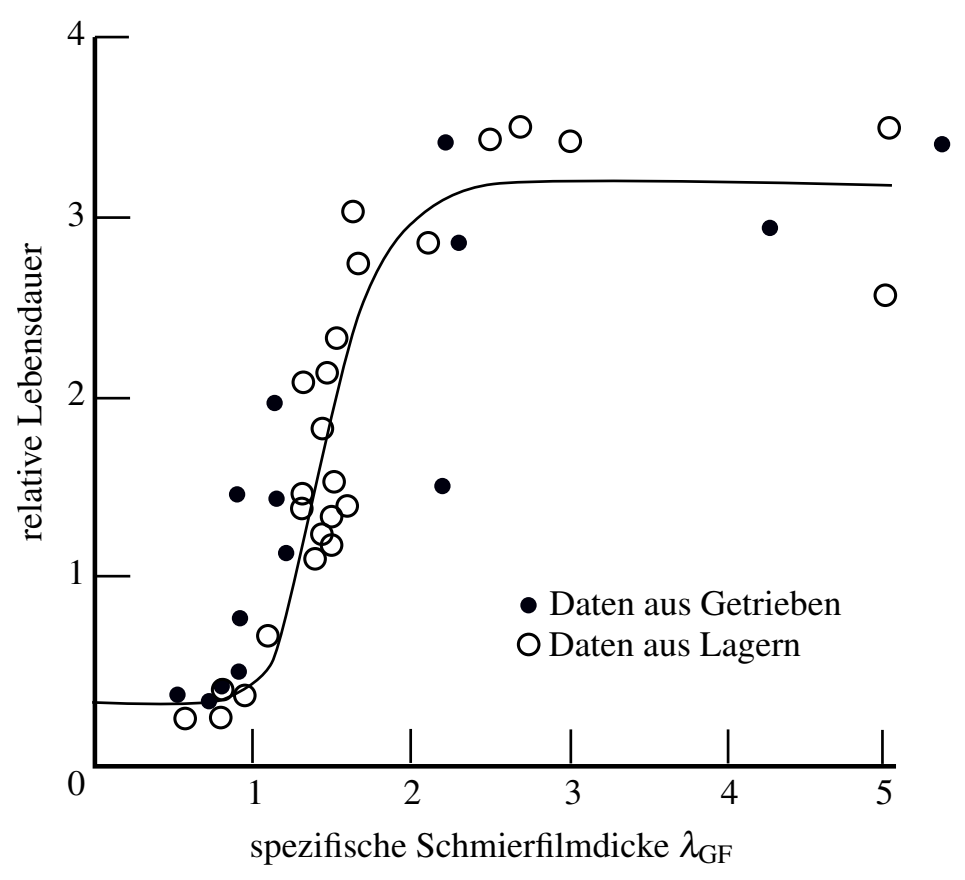

Abbildung 2.5: Einfluss der spezifischen Schmierfilmdicke $\lambda_{\mathrm{GF}}$ auf die Lebensdauer nach Krantz (2014)

(Höhn und Michaelis (2004)). In diesem Fall kann jedoch ein Temperaturbereich existieren, in dem die Schmierfilmdicke bereits kritisch ist, die chemische Aktivität des Additivs jedoch noch nicht ausreichend hoch ist. Zusätzlich kann der additivierte Schmierstoff nicht bei beliebig hohen Temperaturen eingesetzt werden. Daher ist gerade bei Einsatz solcher Schmierstoffe die Kenntnis der Zahnradtemperaturen wichtig, um schadensanfällige Betriebsbereiche zu vermeiden.

Neben der Bewertung der Filmdicke hinsichtlich Verschleiß und Lebensdauer ist $\lambda_{\mathrm{GF}}$ auch für die Entstehung der Reibwärme von Bedeutung. Im Allgemeinen ist der Reibwert $\mu$ für Festkörperreibung wesentlich höher als für Misch- und Flüssigkeitsreibung.

Die Erkenntnisse der EHD können dazu verwendet werden, die Reibwärmeentwicklung im Zahnradeingriff zu berechnen. Die Temperaturen sowohl des Ölfilms als auch des Zahnrads und die Reibleistung sind gekoppelt zu betrachten, wie in Abschnitt 2.2 näher beschrieben wird. Es ist festzuhalten, dass nach der elastohydrodynamischen Theorie die Temperaturen zu Beginn des Eingriffs für die Schmierfilmdicke entscheidend sind (Olver (2002)). Erhöhte Temperaturen von Öl und Zahnrad führen zu geringeren Schmierfilmdicken, was bei gleichbleibender Last und konstanter Oberflächenrauigkeit der Zahnräder die Wahrscheinlichkeit von Mischreibung erhöht. Diese Erkenntnis spiegelt sich auch darin wider, dass die meisten in der Praxis beobachteten Fälle des Zahnradfressens durch zu hohe Temperaturen und nicht etwa durch zu geringe Schmierung auftreten (Townsend und Akin (1983)). Eine genaue Kenntnis des thermischen Zustands zu Beginn des Eingriffs ist demnach unerlässlich, um Schadensprozesse wie Micropitting und Warmfressen ausschließen zu können. Im Umkehrschluss können durch die genaue Kenntnis der Zahnradtemperaturen höhere Leistungsdichten in Getrieben realisiert werden. 


\subsection{Thermalmodellierung von Zahnrädern}

Die im vorangegangenen Abschnitt geschilderten Arbeiten und Erkenntnisse erlauben die Erklärung, Vorhersage und Bewertung der auftretenden Schmierfilme. Die Bestimmung der Reibwärme, die durch die tribologischen Vorgänge im Eingriff entsteht, ist durch die in Abschnitt 2.1.1 erwähnten Arbeiten möglich. Außerdem konnte festgestellt werden, dass die Temperaturen von Zahnrad und Öl kurz vor Eingriff des Zahnrads entscheidend für eine ausreichende Schmierung sind. Im Kontext der Elastohydrodynamik wird dieser Zustand als Einlasszustand bezeichnet. Die Kenntnis der zu erwartenden Temperaturen ist demnach zwingend notwendig, um den tribologischen Zustand im Eingriff bestimmen zu können. Hierfür muss der Wärmehaushalt mit allen Wärmequellen und -senken betrachtet werden. Durch die Kopplung von Reibwärmeentwicklung und gesamtem Temperaturniveau ist der Zusammenhang komplex. Auch in vereinfachten Betrachtungen muss zur Lösung des Wärmeleitungsproblems der Wärmeübergang durch Kühlung berücksichtigt werden. In der Literatur sind einige Ansätze zur Modellierung des Thermalhaushalts von hochbelasteten Zahnrädern bekannt und sollen im Folgenden geschildert werden. Eine Übersicht ist in Tabelle 2.2 dargestellt.

Tabelle 2.2: Literaturstellen zur Thermalmodellierung von Zahnrädern

\begin{tabular}{lcr}
\hline Autor & Art der Untersuchung & Wärmeübergang abgebildet durch \\
Patir und Cheng (1979) & Numerisch & DeWinter und Blok (1974) \\
Handschuh (1992) & Experimentell & - \\
Handschuh und Kicher (1994) & Numerisch & verschiedene $^{1}$ \\
Long et al. (2003) & Exp. und Numerisch & DeWinter und Blok (1974) \\
Jie et al. (2013) & Numerisch & DeWinter und Blok (1974) \\
Xing und Shaojun (2013) & Numerisch & DeWinter und Blok (1974) \\
Wang et al. (2016) & Numerisch & CFD \\
Li und Tian (2017) & Exp. und Numerisch & verschiedene \\
\hline
\end{tabular}

Patir und Cheng (1979) untersuchen in einer der frühesten Arbeiten zu dieser Fragestellung den Thermalhaushalt von Zahnrädern. Die Autoren modellieren das stationäre Temperaturfeld eines Zahnrads unter Einfluss von Reibwärme, Kühlung durch Einspritzkühlung und unter Berücksichtigung des stirnseitigen Wärmeübergangs. In der Arbeit wird ein numerisches Modell zur Bestimmung des Temperaturfeldes beschrieben, dessen Randbedingungen auf Korrelationen aus der Literatur beruhen. Das Modell ist angelehnt an eine Arbeit von Wang (1976) und erweitert das darin beschriebene Modell um Wärmeübergangskoeffizienten an der Zahnradoberfläche. Das Zahnrad wird als periodische Anreihung von Einzelzähnen betrachtet, um den Rechenaufwand gering zu halten. Die Randbedingungen des Finite-Elemente-Modells eines Einzelzahns werden in Zonen der Einspritzkühlung, der ungekühlten Zahnflanken und der Seitenflächen unterteilt. Das schematische Modell des Einzelzahns und die Aufteilung der Randbedingungen, die die Autoren beschreiben, werden in zahlreichen späteren Arbeiten übernommen. Das Modell des Einzelzahns ist in Abbildung 2.6 dargestellt.

Der Wärmeeintrag in die belastete Zahnflanke wird in der Arbeit von Patir und Cheng (1979) 
variiert (Bereich $m$ ). Der Wärmeübergangskoeffizient durch die Einspritzkühlung wird für den gesamten Zahnflankenbereich als konstant angenommen und durch die analytische Korrelation von DeWinter und Blok (1974) bestimmt (Bereiche $t$ und $m$ ). Der Wärmeübergang an den Stirnflächen des Zahnrads wird durch eine Korrelation für rotierende Scheiben angenähert (Bereich $s$ ). Die periodischen Ränder (Bereiche $q$ und $p$ ) werden als adiabate Ränder betrachtet. Die Analyse von Patir und Cheng (1979) erfolgt in dimensionsloser Form.

Die Autoren stellen fest, dass die qualitative Temperaturverteilung stark vom Verhältnis der Wärmeübergangskoeffizienten an den Seitenflächen und den Zahnflanken abhängt. Eine überwiegende Kühlung an den Zahnflanken verursacht demnach starke lokale Gradienten im Zahnbereich, während eine hauptsächlich stirnseitige Kühlung Gradienten über den gesamten Zahn hervorruft, wie in Abbildung 2.7 dargestellt ist.

Die Arbeit von Patir und Cheng (1979) ist dahingehend eingeschränkt, dass der Wärmeübergangskoeffizient als über die gesamte Zahnflanke konstant angenommen wird. Diese Einschränkung begründen die Autoren mit dem Mangel an Daten oder Korrelationen in der Literatur, die den Wärmeübergang ortsaufgelöst beschreiben. Gerade bei hochbelasteten Zahnrädern kann dies dazu führen, dass die auftretenden Temperaturen unterschätzt werden. Die Autoren betonen, dass keine gesicherten Daten für den Wärmeübergang durch Einspritzkühlung verfügbar sind und die Korrelation von DeWinter und Blok (1974) approximativer Natur ist. Die Erkenntnisse der Arbeit beschränken sich daher auf qualitative Beobachtungen. Die Autoren schlussfolgern, dass die quantitative Bestimmung der Temperaturniveaus und -verteilungen nur bei vorhandenen Daten zum Wärmeübergang erfolgen kann.

Long et al. (2003) modellieren die Temperaturverteilung an einem belasteten, geradverzahnten Zahnrad durch das Aufstellen eines FE-Modells und greifen dabei das Modell von Patir und Cheng (1979) auf. Es wird angenommen, dass die Wärmezufuhr durch Reibung und die Kühlung durch die beteiligten Kühlmechanismen simultan ablaufen. Dies ermöglicht es, die Wärmelei-

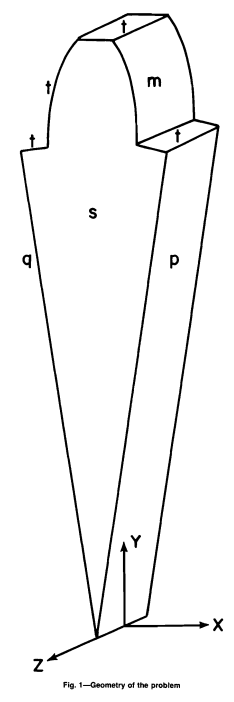

Abbildung 2.6: Periodisches Zahnelement und Wärmeübergangsgebiete nach Patir und Cheng (1979) 
tungsgleichung stationär zu lösen. Während der Seitenwandwärmeübergang auf den gleichen Grundlagen wie in der Arbeit von Patir und Cheng (1979) beruht (Hartnett und Deland (1961), Popiel und Boguslawski (1975) und Dorfman (1963)), wird eine Korrektur eingeführt, um den Einfluss des Zweiphasengemisches aus Luft und Öl in Zahnradumgebung zu berücksichtigen. Dafür wird ein Stoffgemisch definiert, dessen Eigenschaften mit einem Quotienten $d$ zwischen denen von Luft und denen des verwendeten Öls interpoliert werden. Um diesen Quotienten zu bestimmen, verwenden die Autoren Ergebnisse aus einer experimentellen Versuchskampagne. Analog zu Patir und Cheng (1979) wird der Wärmeübergang an den Zahnflanken durch die Korrelation von DeWinter und Blok (1974) abgeschätzt, die zu einem Wärmeübergangskoeffizienten umgeformt wird. Die Reibungswärme, die im Eingriff freigesetzt wird, wird anhand der Getriebekinematik und Reibungsbeiwerten aus experimentellen Arbeiten von Castro und Seabra (1998) und Winter und Michaelis (1984) (Gleichung 2.3) bestimmt und entlang der Zahnkontur aufgelöst ausgewertet. Wie in Kapitel 2.1 beschrieben sind die Schergeschwindigkeit $v_{\mathrm{g}}$ und somit auch der Reibwärmeeintrag ortsabhängig, was erstmalig in der Arbeit von Long et al. (2003) berücksichtigt wird.

Die Autoren vereinfachen das Modell zusätzlich, indem sie ein periodisches Element definieren. Dieses besteht aus einem Einzelzahn eines Zahnrads, das an seinen Schnittflächen adiabate Ränder aufweist. Es werden sieben Oberflächengebiete definiert, an denen jeweils unterschiedliche thermische Randbedingungen angenommen werden. Der Zahn wird aufgeteilt in die belastete und unbelastete Flanke, die Zahnoberseite und den Zahnfuß. Neben den adiabaten Schnittflächen des periodischen Elements werden die Berührfläche mit der Welle und die Stirnseiten des Zahnrads als Ränder definiert.

Long et al. (2003) variieren die Eingangsparameter des Modells und identifizieren so den Einfluss von Zahnlast und Drehzahl auf die Temperaturverteilung im Zahnrad. Dabei zeigt die Zahnradlast den größten Einfluss. Sowohl die mittleren als auch die maximalen Temperaturen
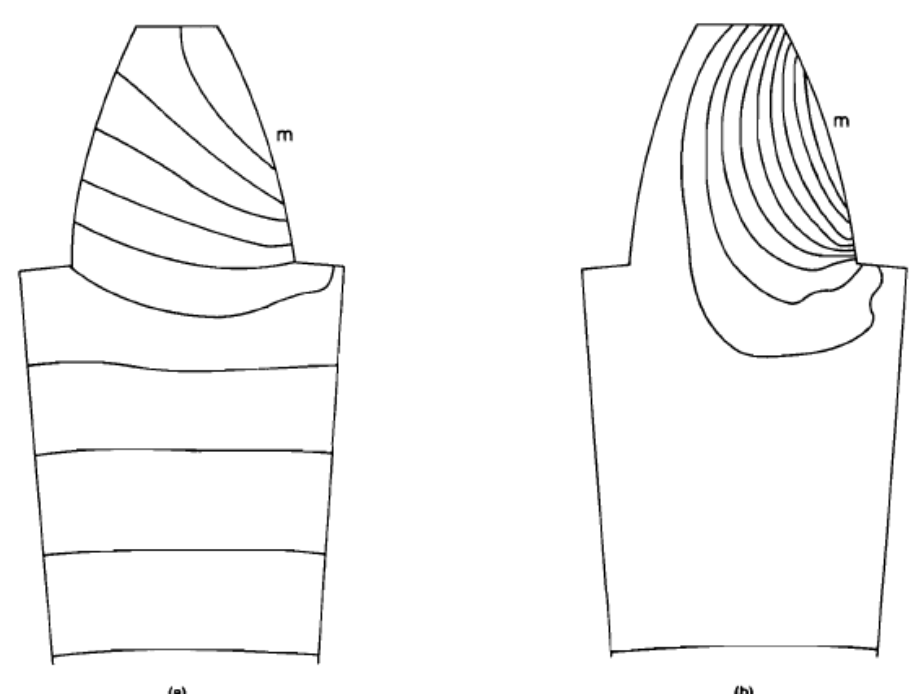

Abbildung 2.7: Isolinien der Temperatur bei dominierendem Seitenwandwärmeübergang (links) und dominierendem Zahnflankenwärmeübergang (rechts) nach Patir und Cheng (1979) 
auf dem Zahnrad steigen mit höheren übertragenen Lasten.

Dadurch, dass der Quotient $d$ iterativ durch die Annäherung der Simulationsergebnisse an die experimentellen Messwerte gewonnen wird, erreichen die Autoren eine Übereinstimmung der simulierten und gemessenen Temperaturen. Dabei liegt der resultierende Wärmeübergangskoeffizient für die Seitenflächen des Zahnrads in einer Größenordnung, die sonst nur in Staupunktnähe bei flüssiger Prallkühlung erreicht wird. Der Wärmeübergangskoeffizient der gekühlten Zahnflanken ist um eine Größenordnung kleiner als der der Seitenflächen.

Jie et al. (2013) beschreiben ein den obenstehenden Ansätzen ähnliches Thermalmodell eines Zahnrads. Die Bestimmung der Randbedingungen erfolgt analog zu den Arbeiten von Patir und Cheng (1979) und Long et al. (2003). Auch Xing und Shaojun (2013) bestimmen die Temperaturverteilung eines belasteten Zahnrads durch die Modellierung eines Einzelzahns. Dabei verwenden sie Korrelationen für rotierende Scheiben für die Seitenflächen, etablierten Zahnreibungskorrelationen für den Reibwärmeeintrag sowie der Wärmeübergangskorrelation für die einspritzgekühlten Flächen von DeWinter und Blok (1974). In beiden Arbeiten wird ein Temperaturmaximum am Ort des Wärmeeintrags beobachtet. Ebenfalls wird in beiden Arbeiten festgestellt, dass die Bestimmung genauer Randbedingungen unerlässlich ist, um verwertbare Abschätzungen der zu erwartenden Temperaturverteilungen treffen zu können.

Wang et al. (2016) modellieren die instationäre Temperaturverteilung an einem belasteten, einspritzgekühlten Zahnrad. Dazu werden zunächst Wärmeübergangskoeffizienten bei vereinfachtem Aufprallen eines Strahls auf die gekühlte Flanke durch numerische Strömungssimulationen berechnet. Der Wärmeübergang an der strömungsabgewandten Flanke wird ebenfalls numerisch bestimmt. Die so gewonnenen Werte werden als Randbedingungen eines zeitlich aufgelösten FE-Modells verwendet, um die instationäre Temperaturverteilung zu berechnen. Dabei werden Temperaturschwankungen von bis zu $17 \mathrm{~K}$ während eines Eingriffszyklus festgestellt.

In einigen Arbeiten wird der Thermalhaushalt von Getrieben und Zahnrädern stark vereinfacht und als thermisches Netzwerk betrachtet (Manin und Play (1999), Koffel et al. (2009), Durand de Gevigney et al. (2012)). Häufig wird in diesen Arbeiten eine Getriebekonfiguration experimentell untersucht, indem charakteristische Temperaturen an den Zahnrädern gemessen und mit den Berechnungen durch die thermischen Netzwerke verglichen werden. Auch hier stellt die Verfügbarkeit von Daten zum Wärmeübergang an Zahnrädern durch Einspritzkühlung eine Lücke dar.

Im Vorangegangenen wurde eine Übersicht über die Literatur zur Thermalmodellierung gegeben. Verschiedene Ansätze und Erkenntnisse zur Bestimmung der Temperaturverteilung an Zahnrädern wurden vorgestellt. Die Arbeit von Patir und Cheng (1979) wird dabei häufig aufgegriffen, indem die Aufteilung der Gebiete des Wärmeübergangs und die verwendeten Korrelationen übernommen werden. Die numerischen Arbeiten, in denen einspritzgekühlte Zahnräder betrachtet werden, verwenden dabei fast ausschließlich die Korrelation von DeWinter und Blok (1974), um den Wärmeübergang an der belasteten Zahnflanke abzubilden. In mehreren Arbeiten wird auf einen Mangel an gesicherten Daten zum Öl-Zahnrad-Wärmeübergang hingewiesen. ${ }^{2}$

\footnotetext{
${ }^{2}$ unter anderem durch Patir und Cheng (1979), Townsend und Akin (1981), Townsend und Akin (1983) und Xing und Shaojun (2013)
} 


\subsection{Prallkühlung an Zahnrädern}

Zur Implementierung der in Abschnitt 2.2 beschriebenen Modelle sind Daten zum Wärmeübergang durch Kühlung erforderlich. Die verfügbaren Daten in der Literatur sind äußerst begrenzt. Die in diesem Zusammenhang bekannten Arbeiten werden im Folgenden zusammengefasst.

In den Thermalmodellen, die im vorangegangenen Abschnitt beschrieben werden, wird häufig auf die Korrelation von DeWinter und Blok (1974) zurückgegriffen. Aufgrund der prominenten Bedeutung dieser Korrelation soll der ihr zu Grunde liegende Ansatz beleuchtet werden. Die Autoren betrachten den Wärmeübergang an einem Zahnrad analytisch, indem sie ein stark vereinfachtes Modell des fluidmechanischen Vorgangs aufstellen. Dazu wird folgendermaßen vorgegangen:

- Die Zahnlücke wird als rechteckige Geometrie betrachtet (siehe Abbildung 2.8).

- Zu Beginn des Vorgangs füllt das Fluid den gesamten Zahnquadranten und ist in Ruhe.

- Die Temperaturdifferenz zwischen Oberfläche und Fluid wird als gering betrachtet. Es wird angenommen, dass daher auch die Viskositätsunterschiede vernachlässigt und die Impuls- und Energiegleichung entkoppelt werden können.

- Die zeitliche Entwicklung der Filmdicke und Geschwindigkeitsverteilung des Fluids werden durch eine analytische Lösung der Impulsgleichung bestimmt. Durch die Annahme konstanter Viskosität ist diese unabhängig vom Wärmeeintrag in das Fluid.

- Um den Wärmeübergang von der Oberfläche in das Fluid zu bestimmen, wird die Energiegleichung numerisch gelöst.

Durch Entdimensionieren und numerisches Lösen der Impulsgleichung und der Energiegleichung bestimmen DeWinter und Blok (1974) die Wärme, die zu einem beliebigen Zeitpunkt durch das Fluid aufgenommen wurde. Daraus lassen sich unmittelbar der zeitliche Verlauf des Wärmeübergangs und seine obere Grenze bestimmen. Die über den gesamten Vorgang des Abschleuderns des Öls maximal abführbare Wärme nach DeWinter und Blok (1974) beträgt

$$
Q_{\mathrm{tot}, \max }=5,6 m \sqrt{\lambda \rho c} \frac{\Delta T}{\sqrt{\omega}}
$$

oder als Wärmeübergangskoeffizient:

$$
h=\frac{\omega Q_{\mathrm{tot}, \max }}{2 \pi \cdot \Delta T \cdot 2,2 m}=2,5454 \sqrt{\lambda \rho c} \frac{\sqrt{\omega}}{2 \pi}
$$

Aus der Betrachtung von Gleichung 2.17 wird bereits die approximative Natur der Korrelation von DeWinter und Blok (1974) deutlich. Durch die getroffene Annahme, dass zu Beginn des Kühlvorgangs die gesamte Zahnlücke stets mit Öl gefüllt ist, findet der eingespritzte Ölvolumenstrom keine Berücksichtigung bei der Bestimmung des Wärmeübergangs. Dadurch, dass das Fluid zu diesem Zeitpunkt als auf der Flanke ruhend angenommen wird, kann auch der Impuls 


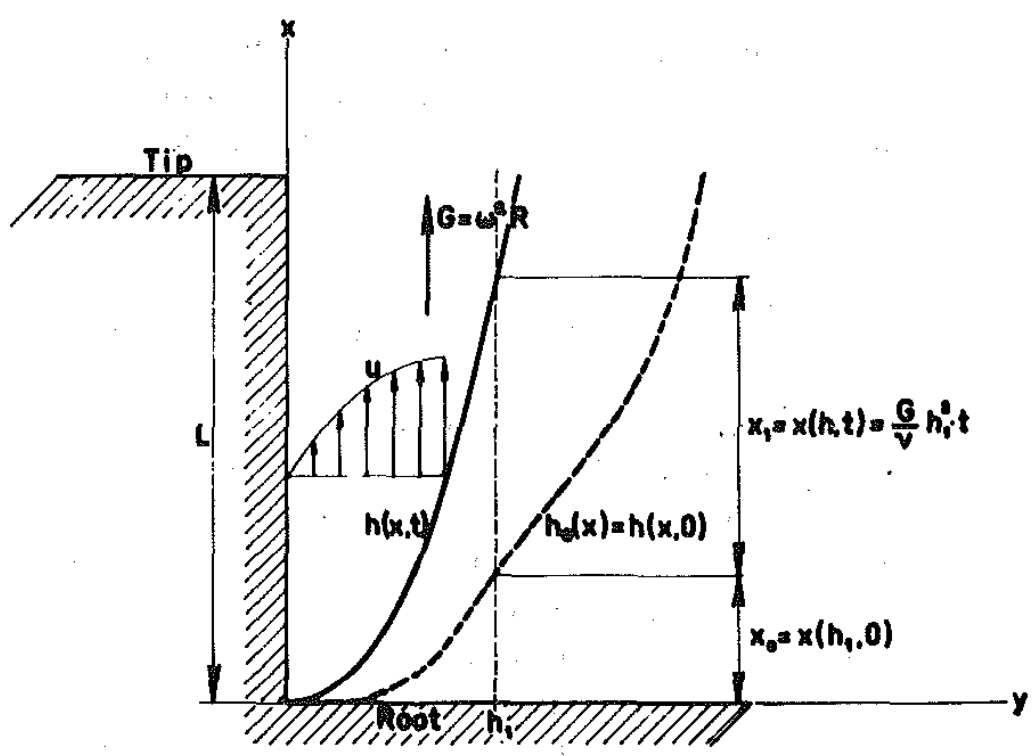

Abbildung 2.8: Auszentrifugieren eines Fluidfilms in einem Zahnquadranten nach DeWinter und Blok (1974)

des auf die Zahnradoberfläche auftreffenden Ölstrahls in Gleichung 2.17 nicht berücksichtigt werden.

Townsend und Akin (1983) untersuchen das Einsetzen von Warmfressen an einem belasteten Zahnradpaar experimentell und analytisch. Die Autoren beschreiben einen Prüfstand, in dem ein Zahnradpaar im Eingriff steht und durch eine Verspannmechanik unter einem einstellbaren Drehmoment bei unterschiedlichen Drehzahlen betrieben werden kann. Zusätzlich werden sowohl der Einspritzdruck des Öls als auch der Düsendurchmesser variiert. In den durchgeführten Experimenten wird die Temperatur der belasteten Zahnflanke unter Verwendung eines Pyrometers gemessen. Dadurch, dass das verwendete Pyrometer zeitlich hochaufgelöste Messungen zulässt und dadurch, dass der Schnittpunkt der Sichtachse des Pyrometers und der Zahnflanke während des Durchlaufs eines Einzelzahns entlang der Flanke wandert, können ortsaufgelöste Temperaturen erfasst werden. Zusätzlich wird ein Thermalmodell vorgestellt, das das Modell von Patir und Cheng (1979) um die Eindringtiefe des Ölstrahls erweitert. Die Eindringtiefe wird dabei durch das vektorielle Modell von Akin et al. (1975) bestimmt. Der Wärmeübergangskoeffizient für Einspritzschmierung wird von der Zahnspitze bis zur Eindringtiefe angenommen. Den Wärmeübergangskoeffizienten, der durch die Korrelation von DeWinter und Blok (1974) bestimmt wird, bewerten die Autoren als zu gering und verwenden daher einen nicht näher beschriebenen eigenen Schätzwert.

Die experimentellen Ergebnisse von Townsend und Akin (1983) zeigen eine Abnahme der Zahnflankentemperaturen mit steigendem Düsenvordruck für alle untersuchten Betriebspunkte. Der Effekt ist dann besonders ausgeprägt, wenn das übertragene Moment oder die Drehzahl hoch sind. Die geringsten Temperaturen am Zahnrad werden dann erreicht, wenn der eingespritzte Ölstrahl den Zahnfuß erreicht. Der beobachtete Zusammenhang zwischen Düsenvordruck und Zahnradtemperatur ist in Abbildung 2.9 für einige gemessene Betriebspunkte von Townsend 
und Akin (1983) dargestellt. Dabei wird ein Moment von 3736 N/cm übertragen. Durchgezogene Linien stellen mittlere Zahnflankentemperaturen, gestrichelte Linien die Minimal- und Maximaltemperaturen dar.

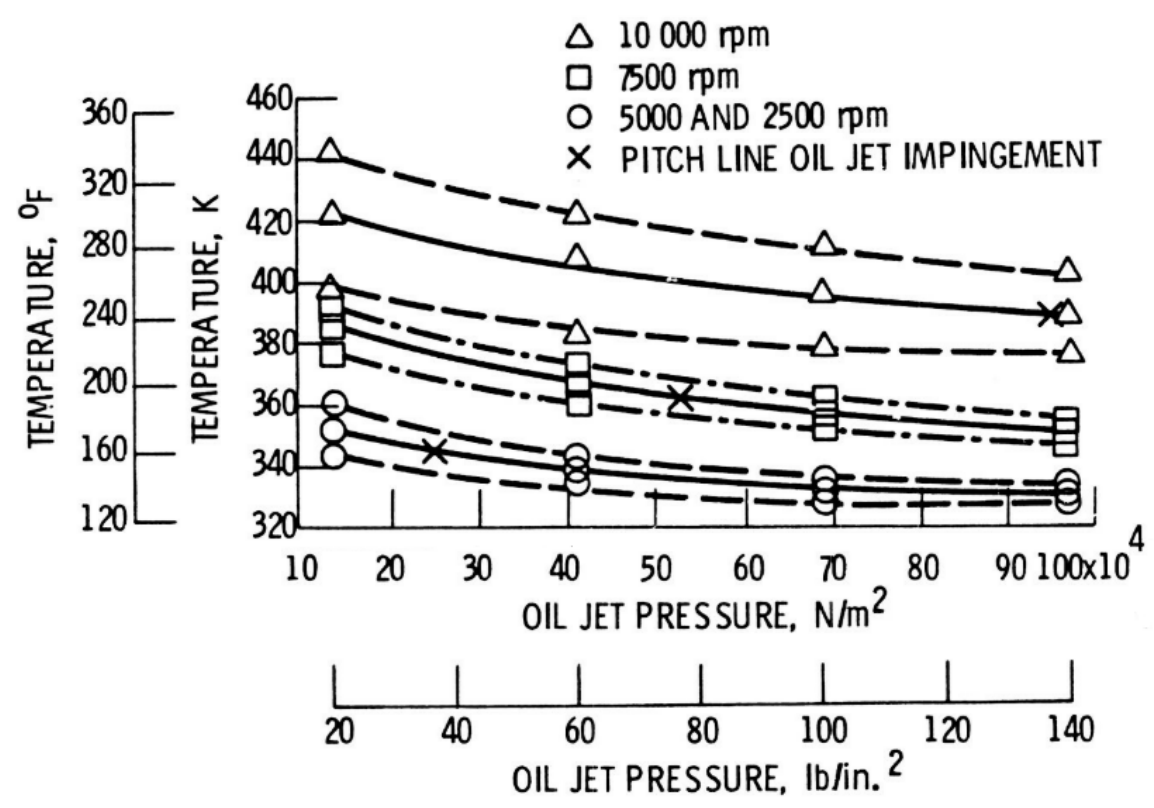

Abbildung 2.9: Einfluss des Düsenvordrucks und des übertragenen Moments auf die Zahnradtemperatur nach Townsend und Akin (1983)

Der Vergleich der experimentellen und numerischen Ergebnisse liefert allgemein übereinstimmende Ergebnisse. Die getroffene Abschätzung des Wärmeübergangs wird jedoch nicht erläutert. Dadurch, dass im Experiment die unbelastete Zahnflanke gekühlt wird, während die Messungen an der belasteten Zahnflanke aufgezeichnet werden, kann die örtliche Verteilung des Wärmeübergangskoeffizienten nicht bestimmt werden.

Schober (1983a) untersucht den Einfluss der Düsenanordnung und der verwendeten Ölmenge auf das Einsetzen von Fressen an einem Zahnradpaar in einem Verspannungsprüfstand. Düsenanordnungen, bei denen Öl in den beginnenden Eingriff, in den auseinandergehenden Eingriff und in radiale Richtung auf den Zahnradumfang gespritzt wird, werden untersucht. Die notwendigen Ölmengen, um Fressen zu vermeiden, sind dabei wesentlich geringer, als die im Vorfeld durch gängige Auslegungsmethoden bestimmten Ölmengen.

Für eine Versuchsreihe mit Einspritzung in den beginnenden Eingriff werden Zahnradtemperaturen unter Last und unter Variation des Ölvolumenstroms gemessen. Dabei führen höhere Ölvolumenströme zu sinkenden Zahnradtemperaturen. Durch Berechnung und Bilanzierung der Verlustleistungen und der abgeführten Wärmeströme bestimmt Schober (1983a) die anteilige Kühlwirkung des Öls. Diese steigt bei höheren Ölvolumenströmen an, während bei geringen Ölvolumenströmen die Kühlung durch Konvektion am Prüfstandsgehäuse und Wärmeleitung durch das Prüftstandsfundament dominiert.

Schober führt die beobachteten geringen Ölmengen zur Vermeidung von Fressen auf die geringen zum vollständigen Aufbau eines tragfähigen EHD-Schmierfilms erforderlichen Ölmengen zurück. Das Einsetzen von Fressen bei geringen Ölvolumenströmen wird von Schober auf kritische 
Zahnradtemperaturen durch geringere Kühlung und nicht auf mangelhafte Ölbenetzung zurückgeführt. Es wird hervorgehoben, dass im Fall sehr geringer Schmiermengen auch geringere Verluste zu erwarten sind.

Handschuh (1992) untersucht die transiente Temperaturverteilung an schrägverzahnten Kegelrädern. Dabei wird ein Fächerstrahl eingesetzt, der die gesamte Zahnbreite abdeckt. Die Zahnräder werden durch eine Verspannmechanik, ähnlich der von Townsend und Akin (1983), vorgespannt und bei unterschiedlichen Drehmomenten und Drehzahlen getestet. Der Einfluss der Position des Ölstrahls wird für insgesamt fünf Positionen um den Umfang des Zahnrads untersucht. Die Oberflächentemperaturen werden ebenfalls durch Pyrometrie gemessen. Die Temperatur wird zusätzlich durch in das Zahnrad eingelassene Thermoelemente erfasst. Auch hier führt erhöhte Ölzufuhr zu geringeren Temperaturen für alle untersuchten Betriebspunkte und Düsenpositionen. Der Temperaturunterschied bei den untersuchten Ölvolumenströmen ist jedoch wesentlich geringer als der, der von Townsend und Akin (1983) beobachtet wird. Die übertragene Leistung hat den stärksten Einfluss auf die resultierenden Temperaturen. Die Temperaturen sind stets an den Zahnspitzen am höchsten und nehmen zum Zahnfuß hin ab. Die Düsenpositionen unmittelbar vor und nach dem Zahneingriff führen zu den niedrigsten Temperaturen und weisen somit die höchsten Wärmeübergänge auf. Es wird berichtet, dass die Einspritzung unmittelbar vor dem Eingriff jedoch mit erhöhten Verlusten durch die Verdrängung des Öls verbunden ist.

Mizutani et al. (1989) bestimmen die Temperaturverteilung eines schnelllaufenden Zahnradpaares und berechnen die anteiligen Beiträge von Reibungs-, Plansch- und Ventilationsverlusten. Sie stellen fest, dass bei hohen Drehzahlen die Plansch- und Ventilationsverluste dominieren. Eine Bestimmung des Wärmeübergangskoeffizienten erfolgt nicht.

Leoni (1991) untersucht die Eignung getrennter Schmierung und Kühlung zur Reduktion der eingespritzten Ölmenge. Durch die Messung der Temperaturen an zwei Stellen eines Zahnrads kann der Wärmestrom durch das Zahnrad näherungsweise bestimmt werden. Aus Bilanzbetrachtungen wird der Wärmeübergangskoeffizient berechnet. Höhere Ölvolumenströme sowie höhere Umfangsgeschwindigkeiten führen in der Untersuchung zu höheren Wärmeübergangskoeffizienten. Die höchsten untersuchten Ölvolumenströme bilden hier eine Ausnahme und führen zu geringeren ermittelten Wärmeübergangskoeffizienten. Aufgrund des Versuchsaufbaus können die Verlustwärme, die im Eingriff entsteht und der Wärmeübergang durch Kühlung nicht voneinander unterschieden werden. Leoni (1991) führt den unerwarteten Rückgang des Wärmeübergangs bei hohen Ölvolumenströmen auf diese Einschränkung zurück.

\subsection{Wärmeübergang durch Prallkühlung}

Zahlreiche technische Anwendungen benötigen Kühlmethoden mit hohen Wärmeübergangskoeffizienten. Der Einsatz von Prallkühlung zur Erzeugung sehr hoher Wärmeübergänge ist effektiv, robust und konstruktiv meist einfach, was zu einer weiten Verbreitung dieser Methode geführt hat. Beim Walzen von Stählen werden sowohl die Walzen als auch das Material durch Prall- und Sprühkühlung gekühlt, um thermische Überlastung zu verhindern und die Prozesstemperaturen zu optimieren (Chen und Tseng (1992)). In der jüngeren Vergangenheit fand durch den steigen- 
den Kühlungsbedarf von Computerchips auch hier Prallkühlung Anwendung (Ebadian und Lin (2011)). Durch zunehmende Miniaturisierung steigen die Anforderungen an die erreichbaren Wärmestromdichten zusätzlich an. In bestimmten militärischen Mikroelektronikanwendungen treten Wärmestromdichten in der Größenordnung von $1 \mathrm{MW} / \mathrm{m}^{2}$ auf. In zukünftigen Anwendungen werden $2,5 \mathrm{MW} / \mathrm{m}^{2}$ erwartet, die durch effiziente Kühlsysteme abgeführt werden müssen. Auch diesen Anforderungen kann durch Prallkühlung begegnet werden.

Im Bereich der Turbomaschinen wird ebenfalls auf Prallkühlung zurückgegriffen, um thermisch hoch belastete Oberflächen und Strukturen zu kühlen. So wird zur Kühlung der Vorderkanten von Laufschaufeln Prallkühlung eingesetzt, da nur im Bereich der Vorderkante durch die typische, geringe Dickenrücklage genügend Bauraum verfügbar ist, ohne die strukturelle Integrität zu gefährden. Bei Leitschaufeln, die keiner Fliehkraftlast standhalten müssen, wird die Prallkühlung auch in der Mitte der Sehnenlänge eingesetzt (Han et al. (2013)).

Wie im vorangegangenen Abschnitt verdeutlicht wird, ist Prallkühlung mittels eines Ölstrahls in Getrieben ab gewissen Leistungsdichten notwendig, um ausreichend hohe Wärmestromdichten abführen zu können. Im Folgenden sollen die Grundlagen der Prallkühlung geschildert werden, um das bereits verfügbare Wissen und die Analogien zum Anwendungsfall aufzuzeigen.

In der Literatur wird das Feld der Prallkühlung häufig unterteilt in Prallkühlung durch

- Gasstrahlen (gas jet impingement)

- Flüssigkeitsstrahlen

in gasförmiger Umgebung (free surface jet, unsubmerged jet)

in flüssiger Umgebung (submerged jet)

- Flüssigkeitsstrahlen mit Phasenübergang.

Stellenweise wird von einphasiger (single phase) bzw. zweiphasiger (two phase) Prallkühlung gesprochen, womit Strömungen ohne bzw. mit Phasenübergang gemeint sind. Die Literatur zur Beschreibung und Vorhersage der Strömung und des Wärmeübergangs durch Gasstrahlen ist umfangreich und wird von Downs und James (1987) sowie Han und Goldstein (2001) zusammengefasst. Im vorliegenden Fall ist ausschließlich die Kühlung durch Flüssigkeitsstrahlen in gasförmiger Umgebung relevant. Einige wichtige Aspekte dieser Form der Prallkühlung sollen im Folgenden umrissen werden. Für eine ausführliche Darstellung sei auf die Übersichtsarbeit von Webb und Ma (1995) verwiesen.

In Arbeiten zur Prallkühlung werden die Strömung und der Wärmeübergang meist durch dimensionslose Kennzahlen beschrieben. Bei Rundstrahlen ist die Definition der Reynoldszahl mit der mittleren Strahlgeschwindigkeit $u_{\mathrm{s}}$, dem Austrittsdurchmesser des Rundstrahls $d$ und der kinematischen Viskosität $v$ üblich, als:

$$
\operatorname{Re}_{d}=\frac{u_{\mathrm{s}} d}{v}
$$


Die Viskosität, die Wärmeleitfähigkeit, die Dichte und die spezifische Wärmekapazität des Fluids werden über die Prandtlzahl ausgedrückt, mit:

$$
\operatorname{Pr}=\frac{v}{a}
$$

und der sogenannten Temperaturleitfähigkeit $a=\frac{\lambda}{\rho c}$. Der Wärmeübergangskoeffizient $h$ kann durch die Nußeltzahl dimensionslos ausgedrückt werden:

$$
\mathrm{Nu}=\frac{h d}{\lambda} .
$$

Darin wird im Zusammenhang von Rundstrahlen $d$ als charakteristische Länge eingesetzt, $\lambda$ ist die Wärmeleitfähigkeit des Fluids.

\subsubsection{Prallkühlung durch Flüssigkeitsstrahlen}

Die hohen Wärmeübergangskoeffizienten der Prallkühlung werden erreicht, indem Konvektion erzwungen wird. Durch den Strahlaufprall an der zu kühlenden Oberfläche wird die Strömung umgelenkt, es werden hohe Geschwindigkeitsgradienten in Wandnähe hervorgerufen. Die Eigenschaften der sich durch Wandreibung ausbildenden Strömungsgrenzschicht und der damit gekoppelten thermischen Grenzschicht sind entscheidend für den erreichbaren Wärmeübergang.

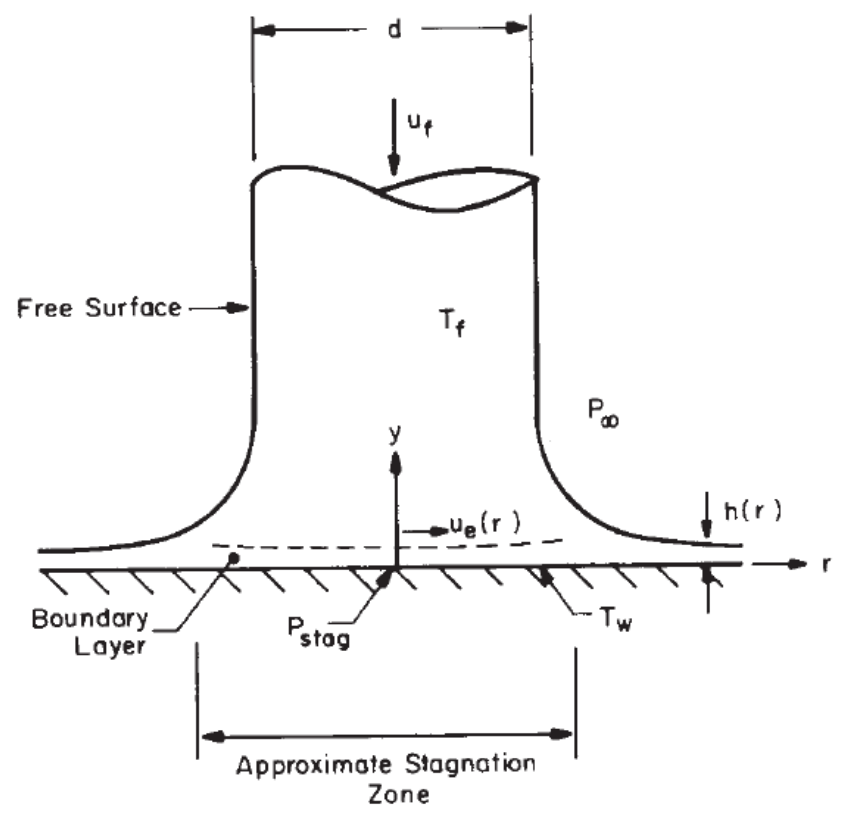

Abbildung 2.10: Ausbildung einer Grenzschicht in der Stauströmung durch Prallkühlung mit einem unbewegten, flüssigen Rundstrahl in Gasumgebung nach Lienhard (2006)

Durch Reibung an der Wand und den dadurch entstehenden Impulsverlust in der Strömung nimmt die Dicke der Strömungsgrenzschicht eines Prallstrahls in Strömungsrichtung zu. Im Gegensatz zur Umströmung einer ebenen Platte oder von Tragprofilen kann die Grenzschicht 
jedoch nicht ungehindert anwachsen. Bei achsensymmetrischen Prallstrahlen nimmt die Dicke des Flüssigkeitsfilms aus Kontinuitätsgründen stetig ab (siehe Abbildung 2.10). An einem bestimmten Radius erreicht die Dicke der Strömungsgrenzschicht die Filmhöhe. Ab diesem Punkt ist die gesamte Strömung als reibungsbehaftet zu betrachten. Zusätzlich kann die Strömung an einem weiter vom Staupunkt entfernten Radius einen hydraulischen Sprung erfahren, an dem sie vom schießenden Zustand durch eine schlagartige Dickenzunahme in den strömenden Zustand übergeht. Durch die genannten Phänomene sind die Beschreibung und Vorhersage der Strömung und des Wärmeübergangs komplex. In der Literatur wird der Wärmeübergang durch Prallkühlung daher in Bereiche unterteilt, die sich durch den Charakter ihrer Strömung unterscheiden. Die Unterteilung ist in Abbildung 2.11 dargestellt.

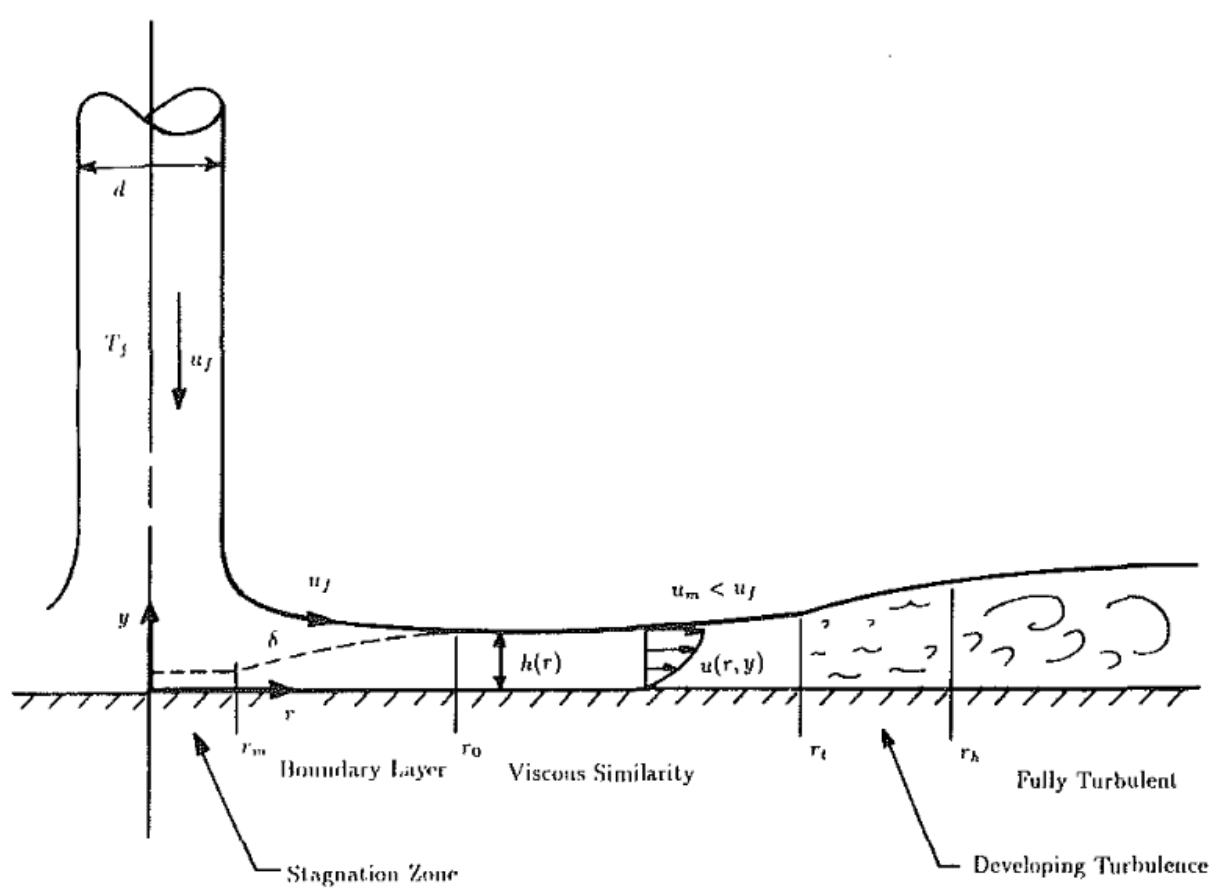

Abbildung 2.11: Unterteilung eines auftreffenden Prallstrahls in charakteristische Strömungsgebiete nach Liu et al. (1991)

Verschiedene Arbeiten befassen sich mit der Strömung und dem Wärmeübergang in einzelnen der in Abbildung 2.11 dargestellten Bereiche. Liu et al. (1991) bestimmen den Wärmeübergang durch Prallkühlung in der Stauzone von Flüssigkeitsstrahlen gleichförmigen Geschwindigkeitsprofils anhand analytischer Überlegungen zu

$$
\mathrm{Nu}=\left\{\begin{array}{lc}
0,715 \operatorname{Re}_{d}^{0,5} \operatorname{Pr}^{0,4}, & 0,15<\operatorname{Pr} \quad<3 \\
0,797 \operatorname{Re}_{d}^{0,5} \operatorname{Pr}^{\frac{1}{3}}, & 3 \leq \operatorname{Pr}
\end{array} .\right.
$$

Dies steht in guter Übereinstimmung mit den Ergebnissen von Ma et al. (1996), die den Wärmeübergang an Oberflächen unter Berücksichtigung inhomogener Randbedingungen analytisch bestimmen. Der Einfluss der Randbedingungen wird dabei über einen Faktor $\varepsilon$ berücksichtigt. 
Unter homogenen Wärmestromrandbedingungen gilt $\varepsilon=1$ und somit nach Ma et al. (1996)

$$
\mathrm{Nu}=\left\{\begin{array}{l}
0,7212 \operatorname{Re}_{d}^{0,5} \operatorname{Pr}^{0,4}, \quad 0,7<\operatorname{Pr} \quad<3 \\
0,7212 \operatorname{Re}_{d}^{0,5} \operatorname{Pr}^{0,37}, \quad 3<\operatorname{Pr}<10 \\
0,8597 \operatorname{Re}_{d}^{0,5} \operatorname{Pr}^{\frac{1}{3}}, \quad 10<\operatorname{Pr}
\end{array} .\right.
$$

Durch Gleichungen 2.21 und 2.22 ist der Wärmeübergang im Staupunktbereich durch einfache Ausdrücke und für die Prandtlzahlen der meisten Fluide, die in technischen Anwendungen eingesetzt werden, bestimmbar. Der Wärmeübergang in den stromab anknüpfenden Bereichen hängt in großem Maße vom Wärmeübergang im Staupunktbereich ab. Durch die außerhalb des Staupunktbereichs anwachsenden Grenzschichten nimmt der Wärmeübergang stetig ab. Lediglich durch den Umschlag zur turbulenten Strömung kann ein positiver Gradient des lokalen Wärmeübergangs in Strömungsrichtung beobachtet werden. Analog zu Gleichungen 2.21 und 2.22 sind für die Bereiche stromab des Staupunkts analytische Ausdrücke zur Bestimmung des Wärmeübergangs bekannt. Da für laminare und turbulente Strömungen und verschiedene Bereiche der Prandtlzahl unterschiedliche, teils sehr komplexe Ausdrücke zur Anwendung kommen, ist die Darstellung der Theorie umfangreich und soll im Rahmen dieser Arbeit nicht erfolgen. Ein ausführlicher Überblick wird von Webb und Ma (1995) gegeben. Nahezu allen Korrelationen für die unterschiedlichen Bereiche der Strömung ist jedoch der Einfluss der Reynoldszahl auf den Wärmeübergang gemeinsam, je nach Fall entweder zu

$$
\mathrm{Nu} \propto \mathrm{Re}^{0,5}
$$

oder zu

$$
\mathrm{Nu} \propto \mathrm{Re}^{0,333}
$$

Aufgrund des hohen Dichteverhältnisses zwischen Flüssigkeit und Luft in den meisten Anwendungsfällen ist die Beeinflussung des Strahls durch die ihn umgebende Luft gering. Dadurch ist der Einfluss des Abstands zwischen Düse und Oberfläche gering. Stevens und Webb (1991) berichten eine Abhängigkeit des Wärmeübergangs in Abhängigkeit des Düsenabstands von der Oberfläche, $z$, mit

$$
\mathrm{Nu} \propto\left(\frac{z}{d}\right)^{-0,052}
$$

\subsubsection{Prallkühlung an bewegten Oberflächen}

Der Wärmeübergang durch Prallkühlung an bewegten Oberflächen wird in einigen Arbeiten diskutiert. Carper et al. (1986) beschreiben den gemittelten Wärmeübergang an einer rotierenden Scheibe bei konzentrischen Rotations- und Strahlachsen durch den Ausdruck

$$
\overline{\mathrm{Nu}}_{D}=0,097 \operatorname{Re}_{\omega}^{0,384} \operatorname{Re}_{d}^{0,459} \operatorname{Pr}^{0,448}
$$

mit der Rotationsreynoldszahl

$$
\operatorname{Re}_{\omega}=\frac{\omega D^{2}}{v} .
$$


Aus Gleichung 2.26 ist ersichtlich, dass eine Steigerung der Oberflächengeschwindigkeit orthogonal zur Strahlachse den flächen- und zeitgemittelten Wärmeübergang erhöht. Dies liegt vor allem an den dünneren Grenzschichten, die sich durch die laterale Bewegung der Oberfläche relativ zum Strahl ausbilden. Eine Erhöhung der Strahlreynoldszahl $\operatorname{Re}_{\mathrm{d}}$ erhöht ebenfalls den mittleren Wärmeübergang an der Scheibe mit $\mathrm{Nu} \propto \mathrm{Re}_{\mathrm{d}}^{0,459}$. Somit liegt trotz grundsätzlich anderer Zustände ein vergleichbarer Einfluss der Strahlgeschwindigkeit im Vergleich zur Prallkühlung unbewegter Oberflächen vor. Lavalle et al. (2007) bestätigen die Korrelation von Carper et al. (1986) durch experimentelle Daten mit Abweichungen im Bereich von etwa 20\%. Gleiches gilt für das Auftreffen eines Strahls außerhalb des Mittelpunkts der Scheibe mit

$$
\overline{\mathrm{Nu}}_{D}=0,57 \operatorname{Re}_{\omega}^{0,272} \operatorname{Re}_{d}^{0,442} \operatorname{Pr}^{0,381}(1-2 r / D)^{0,255} .
$$

\subsubsection{Instationäre Prallkühlung}

In den vorangegangenen Betrachtungen konnte stets von stationären Strömungszuständen und thermischen Randbedingungen und somit zeitlich konstanten Wärmeübergängen ausgegangen werden. Im Kontext dieser Arbeit sind jedoch periodisch instationäre Zustände zu erwarten. Zumbrunnen und Aziz (1993) untersuchen die instationäre Prallkühlung mit dem Ziel, den erreichbaren Wärmeübergang zu erhöhen. Die Autoren beschreiben einen Versuchsaufbau, in dem ein flüssiger, ebener Freistrahl durch rotierende Schneiden periodisch unterbrochen wird. Als eben gelten dabei Freistrahlen, wenn ihre Tiefenausdehnung wesentlich größer als ihre Breitenausdehnung ist. Durch den Vergleich der Wärmeübergangskoeffizienten im stationären und im intermittierenden Fall wird eine Erhöhung des mittleren Wärmeübergangs festgestellt. Dabei stellen die Autoren kein asymptotisches Verhalten des Wärmeübergangs mit steigender Frequenz der Intermittenz fest, wie es bei zunehmender Turbulenz des Strahls der Fall ist. Außerdem wird im gesamten betrachteten Bereich der Oberfläche eine Erhöhung des Wärmeübergangs beobachtet, während bei Wärmeübergangssteigerungen durch Turbulenzerhöhung des Strahls nur lokale Erhöhungen beobachtet werden.

Bei vollständig intermittierenden Strahlen, bei denen die Strömung kurzzeitig unterbrochen wird, kann eine Erhöhung des mittleren Wärmeübergangs beobachtet werden. Maximale Steigerungen des Wärmeübergangs von 33\% (Zumbrunnen und Aziz (1993)) bei Flüssigkeitsstrahlen sowie 30\% (Herwig und Middelberg (2008)) und 15\% (Herwig et al. (2004)) bei Gasstrahlen werden beobachtet. Dabei wird der Wärmeübergang durch instationäre Strahlen stets im Verhältnis zur stationären Anströmung mit der maximalen Geschwindigkeit, die im instationären Fall auftritt, verglichen. Jedoch können zeitlich veränderliche Strahlen den mittleren Wärmeübergang auch reduzieren. Im Fall von Strahlen, deren Geschwindigkeitsverlauf einen Sinusverlauf annimmt, werden Rückgänge des Wärmeübergangs von 20\% (Sheriff und Zumbrunnen (1994)) beobachtet. In diesem Fall überwiegt der ungewünschte Effekt der Unterbrechung der Fluidzufuhr und damit des Wärmeübergangs den günstigen Effekt der geringeren Grenzschichtdicke.

Das Auftreten von transienten Zuständen der Oberflächentemperaturen der thermischen Grenzschicht führt ebenfalls zu starken Änderungen des Wärmeübergangs im Vergleich zu stationären Zuständen (Zumbrunnen (1992)). Dieser Effekt wird vor allem bei kleinen Prandtlzahlen beob- 


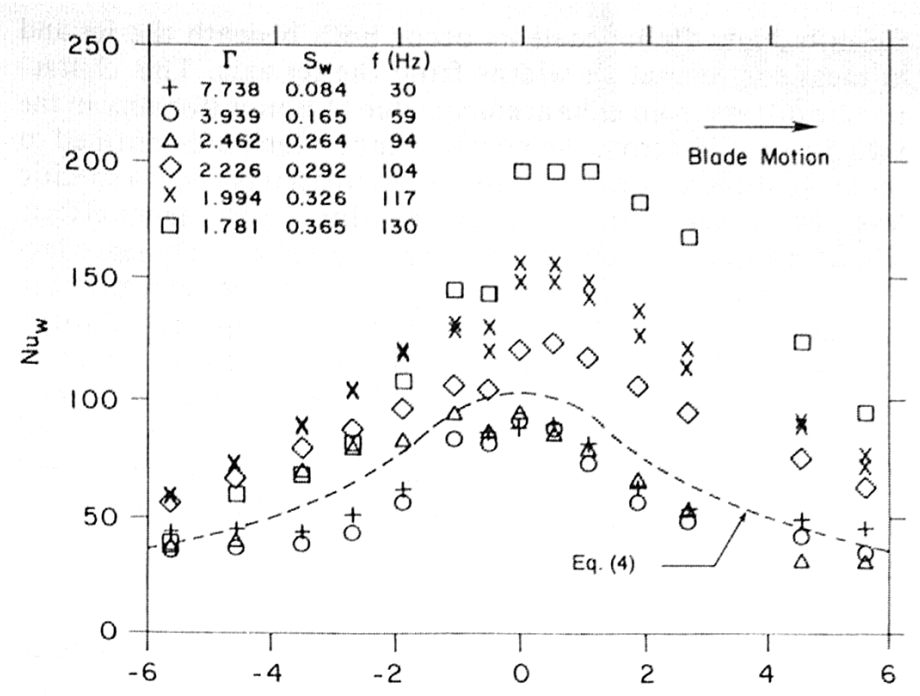

Abbildung 2.12: Verteilung des Wärmeübergangs durch intermittierende Prallkühlung bei unterschiedlichen Unterbrechungsfrequenzen nach Zumbrunnen und Aziz (1993)

achtet, da bei $\operatorname{Pr}<1$ auch die freie Außenströmung maßgeblich am Wärmetransport beteiligt ist.

\subsubsection{Zusammenfassung}

Aus dem Überblick über die verfügbare Literatur ist ersichtlich, dass der Wärmeübergang durch stationäre, achsensymmetrische, orthogonale Prallstrahlen gut beschrieben ist. Der isolierte Einfluss verschiedener Abweichungen von diesem grundlegenden Vergleichsfall wird ebenfalls in einigen Arbeiten behandelt. So sind der Wärmeübergang durch pulsierende Strahlen und die Kühlung bewegter Oberflächen in gewissen Grenzen mathematisch beschrieben. Auch die hier nicht dargelegte Anströmung unter einem nicht orthogonalen Winkel wird durch einige Arbeiten beschrieben. Die folgenden Einzeleffekte sind bekannt:

- Der Wärmeübergang durch Prallkühlung verhält sich in weiten Bereichen proportional zu $\operatorname{Re}_{\mathrm{d}}^{j}$ mit $0,333 \leq j \leq 0,5$.

- Zeitliche Schwankungen des Strahls können den mittleren Wärmeübergang erhöhen, sofern es sich um eine intermittierende Strömung handelt. Sinusmodulierte Geschwindigkeitsschwankungen führen zu geringeren mittleren Wärmeübergängen.

- Der Einfluss des Anstellwinkels des Strahls auf den Wärmeübergang ist gering. Prallstrahlen, die unter einem Winkel von unter $90^{\circ}$ auftreffen, führen zu geringeren Wärmeübergangskoeffizienten.

Bei der Prallkühlung von Zahnrädern sind alle genannten Effekte zu erwarten. Die Bestimmung des zu erwartenden Wärmeübergangs durch die Öl-Zahnrad-Interaktion ist nach dem 
derzeitigen wissenschaftlichen Kenntnisstand nicht möglich, da keine Informationen über die Wechselwirkungen zwischen den jeweiligen Effekten vorliegen.

\subsection{Zielsetzung dieser Arbeit}

In Abschnitt 2.1 wird dargestellt, weshalb die Schmierung und Kühlung von Getrieben unerlässlich ist, gleichzeitig aber besonders für schnelldrehende Getriebe zu erheblichen Verlusten führen kann. Daher sind ein sparsamer Umgang und eine möglichst effiziente Zufuhr des Kühlmittels unerlässlich. Die Elastohydrodynamik, die in Abschnitt 2.1.2 beschrieben wird, zeigt jedoch, dass nur durch die Einhaltung auslegungsspezifischer Temperaturmaxima auf der Zahnradoberfläche Schäden an den Zahnrädern vermieden und somit ausreichende Lebensdauer sichergestellt werden kann. Dazu muss der Thermalhaushalt der Zahnräder verstanden und dementsprechend konzipiert werden. Abschnitte 2.2 und 2.3 zeigen auf, dass der gegenwärtige wissenschaftliche Kenntnisstand dazu nicht ausreicht. Die meisten bekannten Modellierungsansätze greifen auf die Korrelation von DeWinter und Blok (1974) zurück, um den durch die Einspritzkühlung hervorgerufenen Wärmeübergang abzuschätzen. Die Ergebnisse experimenteller Untersuchungen belasteter Zahnradpaare können stets nur mäßig mit den aufgestellten Modellen in Einklang gebracht werden. Dies liegt vor allem darin begründet, dass die Korrelation von DeWinter und Blok (1974) analytischer Natur ist und auf äußerst weitreichenden Vereinfachungen der Strömungs- und Wärmetransportvorgänge beruht.

Eine experimentelle Datenbasis zum Zahnradwärmeübergang existiert nicht. Die bekannten experimentellen Untersuchungen erlauben in keinem Fall die Trennung zwischen der Reibwärme, die im Zahnradeingriff erzeugt wird und dem Wärmeübergang durch Einspritzkühlung. Die Berechnung der Wärmeübergangskoeffizienten für die veröffentlichten experimentellen Daten durch das Berücksichtigen des Reibwärmeeintrags ist nicht möglich. Durch die Elastohydrodynamik kann der Reibwärmeeintrag nur näherungsweise bestimmt werden. Die weiteren thermischen Randbedingungen der Untersuchungen sind in keinem der Fälle genau genug bekannt. Örtlich aufgelöste Wärmeübergangskoeffizienten sind ebenfalls nicht aus den Daten bestimmbar.

Aus der Zusammenfassung der Arbeiten zur Prallkühlung in Abschnitt 2.4 wird deutlich, dass der Wärmeübergang für einige generische Fälle eingehend beschrieben wird. Auch einzelne Einflüsse, wie Prallkühlung durch Flüssigkeitsstrahlen unter einem Winkel, sind innerhalb gewisser Grenzen beschrieben. Dadurch, dass im Fall der Einspritzkühlung in Getrieben jedoch mehrere Effekte simultan auftreten, reicht der Stand der Wissenschaft nicht zur Bestimmung des Öl-Zahnrad-Wärmeübergangs aus.

Um die Entwicklung hochbelasteter Getriebe zu ermöglichen, muss dieser Mangel behoben und der Wärmeübergang zwischen Kühlmittel und Zahnrad experimentell ermittelt werden. Dies ist einerseits notwendig, um eine effiziente Auslegung derartiger Getriebe zu gewährleisten. Andererseits sind experimentelle Daten unerlässlich, um CFD-Modelle der Öl-Zahnrad-Interaktion und des Öl-Zahnrad-Wärmeübergangs zu validieren.

Die vorliegende Arbeit schließt diese Lücke. Dazu werden eine experimentelle Methode und eine Versuchsanordnung vorgestellt, mit welchen der Wärmeübergang an einem einspritzgekühlten 
Zahnrad bestimmt werden kann. Die Bestimmung des Wärmeübergangs erfolgt örtlich aufgelöst. Thermographische, örtlich hochaufgelöste Messungen der Oberflächentemperatur, die häufig zur Bestimmung des Wärmeübergangs genutzt werden, sind im vorliegenden Fall nicht möglich. Daher wird in dieser Arbeit eine Methode vorgestellt, durch die der Wärmeübergang mit einer örtlich begrenzten Anzahl von Messpunkten bestimmt werden kann. Die Messunsicherheit der vorgestellten Methode wird statistisch untersucht. Eine Versuchsanordnung, mit der der Wärmeübergang am Zahnrad unter Laborbedingungen erzeugt werden kann, wird vorgestellt.

Zur Erfüllung der Zielsetzung wird folgendermaßen vorgegangen:

1. Identifikation der relevanten Parameter für den Öl-Zahnrad-Wärmeübergang

2. Entwicklung einer experimentellen Methode zur Messung des Wärmeübergangs und Bestimmung ihrer Messunsicherheit

3. Umsetzung der Methode in einem Versuchsaufbau

4. Charakterisierung des Öl-Zahnrad-Wärmeübergangs unter Variation der Parameter

5. Ableitung einer Korrelation zur Abbildung des mittleren Wärmeübergangs 


\section{Untersuchte Öleinspritzkühlung}

Die Analyse des Stands der Wissenschaft in Kapitel 2 zeigt, dass der Kenntnisstand zum Wärmeübergang durch Einspritzkühlung lückenhaft ist. Die wenigen existierenden Arbeiten zum Öl-Zahnrad-Wärmeübergang befassen sich zudem mit unterschiedlichen konstruktiven Ausgestaltungen der Öleinspritzung, weshalb ein Vergleich nur qualitativ möglich ist. So ist die Arbeit von Handschuh (1992) die einzige Arbeit zum Wärmeübergang durch aufgefächerte Ölstrahlen. Andere Arbeiten (Townsend und Akin (1981), Townsend und Akin (1983), Akin und Townsend (1985)) beschreiben den Wärmeübergang durch zylindrische Düsen bzw. Ölstrahlen. Die Korrelation von DeWinter und Blok (1974) wird mit der Modellvereinfachung abgeleitet, dass der Raum zwischen zwei Zahnflanken nach Einspritzen durch die Düse mit Öl aufgefüllt ist, wodurch die Form der Ölzufuhr außerhalb der Betrachtung liegt. Darüber hinaus wird in den bekannten Quellen die geometrische Ausgestaltung meist nur schemenhaft geschildert.

Um die Zielsetzung der vorliegenden Arbeit zu erfüllen, wird der Wärmeübergang an einem einspritzgekühlten Zahnrad untersucht. Um die Ergebnisse in den Kontext der wissenschaftlichen Literatur einordnen zu können und nachfolgenden Arbeiten die Anknüpfung zu ermöglichen, soll in diesem Kapitel die untersuchte Öleinspritzkühlung charakterisiert werden. Insbesondere für numerische Untersuchungen soll so eine Validierungsbasis geschaffen werden, die bisher nicht existiert.

Zum derzeitigen Punkt sind keine Arbeiten bekannt, in denen der Öl-Zahnrad-Wärmeübergang isoliert von anderen Wärmeströmen bestimmt wird. Es ist keine Systematik bekannt, nach der die geometrischen und strömungsmechanischen Parameter der Kühlung klassifiziert werden können. Zur Erfüllung der Zielsetzung dieser Arbeit müssen daher in einem ersten Schritt die für den Öl-Zahnrad-Wärmeübergang relevanten Parameter identifiziert werden. Dies soll im Folgenden anhand der untersuchten Öleinspritzung geschehen. Anschließend werden die physikalischen Parameter betrachtet, die einen Betriebspunkt definieren. Danach werden die zur Beschreibung des Ölaufpralls verwendeten abgeleiteten Größen eingeführt.

\section{1 Öleinspritzkühlung}

\section{Geometrische Parameter}

Die betrachtete Kühlmethode nutzt die hohen erreichbaren Wärmeübergangskoeffizienten der Prallkühlung, indem Öl in Form eines Ölstrahls direkt auf die zu kühlende Zahnradoberfläche gespritzt wird. Dazu wird eine Ölbrause verwendet, die aus mehreren identischen Öldüsen und einer Zuführbohrung besteht. Diese ist in geringem Abstand zum Zahnrad angeordnet. Die Anordnung ist in Abbildung 3.1 dargestellt.

Die untersuchte Ölbrause besitzt vier Düsen, die gleichmäßig über die Zahnbreite $W=90 \mathrm{~mm}$ verteilt sind. Das Öl wird über die Zuführbohrung zu den Düsen geführt. Diese ist wesentlich größer als die Düsenbohrungen, um die Strömungsgeschwindigkeit gering zu halten. Dies sorgt dafür, dass an allen Düseneinlässen nahezu identische Anströmbedingungen herrschen und 


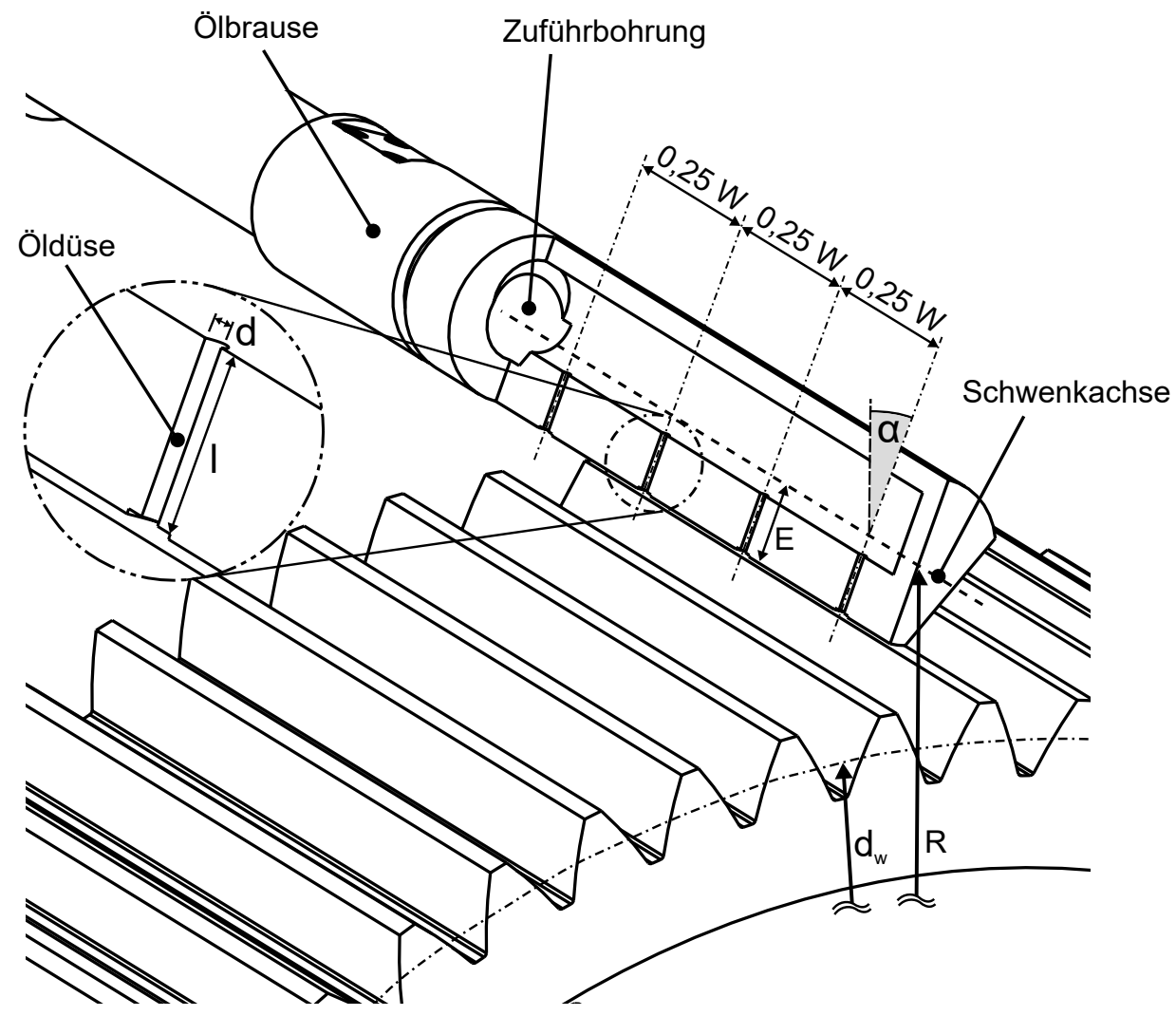

Abbildung 3.1: Charakteristische geometrische Parameter der untersuchten Öleinspritzkühlung

die sich ausbildenden Ölstrahlen nur äußerst geringe Unterschiede in ihren Strömungsprofilen aufweisen. Der Abstand der Düsen zueinander beträgt 0,25W, der Abstand der äußeren Düsen zu den Stirnseiten des Zahnrads beträgt 0,125W. Die Düsen sind als Bohrungen mit Durchmesser $d=1,15 \mathrm{~mm}$ und einer Länge von $l=7 \mathrm{~mm}$ ausgeführt.

Die Strömung des Ölstrahls in der Düse ist entscheidend für den Aufprallvorgang auf dem Zahnrad und somit für den Wärmeübergang. Sie wird vor allem durch die Düsengeometrie beeinflusst, wie in Abschnitt 3.2 erläutert wird. Maßgeblichen Einfluss haben der Bohrungsdurchmesser $d$ sowie das mit der Länge der Bohrung, $l$, gebildete Verhältnis $\frac{l}{d}$, das im vorliegenden Fall 6,09 beträgt.

Aufgrund der gleichmäßigen Aufteilung der Düsen sind für jedes Zahnviertel identische Vorgänge bezüglich des Ölaufpralls und des Wärmeübergangs zu erwarten. Dies ermöglicht die Übertragbarkeit auf Fälle, in denen eine periodische Anordnung von Einzeldüsen betrachtet wird. Unter der Voraussetzung, dass das Aufeinandertreffen zweier Ölfronten keine stromauf gerichtete Rückwirkung hat, gilt dies auch für das Auftreffen eines einzelnen Ölstrahls. Zur Bewertung der Übertragbarkeit kann das Verhältnis aus Düsendurchmesser und Breite des periodischen Elements herangezogen werden, das in der betrachteten Anordnung

$$
\xi=\frac{0,25 W}{d}=19,6
$$


beträgt. Zur Umsetzung der in Kapitel 4 beschriebenen Methode wird die Periodizität der Anordnung ebenfalls ausgenutzt.

Die Strömung der Luft, die das Zahnrad umgibt, interagiert mit dem Ölstrahl. Neben der Oberflächengeschwindigkeit des Zahnrads, das diese Strömung anregt, ist die Länge der Strecke, die der Ölstrahl zwischen Düsenaustritt und Zahnrad zurücklegt, entscheidend für die Interaktion zwischen Luft und Öl (Akin et al. (1975), Krug (2018)). Sie wird bestimmt durch den Abstand $R$ zwischen der Schwenkachse der Düse und der Zahnradachse, der Exzentrizität $E$ des Düsenaustritts, dem Teilkreisdurchmesser $d_{\mathrm{w}}$ des Zahnrads und dem Düsenwinkel $\alpha$. Der Düsenwinkel ist der Winkel zwischen der Bohrungsachse einer Öldüse und der Ebene, die durch die Schwenkachse der Ölbrause und die Zahnradachse aufgespannt wird (siehe Abbildung 3.1). Eine Übersicht der geometrischen Parameter, die die radiale Öleinspritzkühlung definieren, ist in Tabelle 3.1 dargestellt.

Tabelle 3.1: Geometrische Parameter der untersuchten Einspritzkühlung

\begin{tabular}{lcr}
\hline Parameter & Symbol & Wert \\
\hline Düsenradius & $R$ & $153 \mathrm{~mm}$ \\
Düsendurchmesser & $d$ & $1,15 \mathrm{~mm}$ \\
Länge der Düsenbohrung & $l$ & $7 \mathrm{~mm}$ \\
Exzentrizität des Düsenauslasses & $E$ & $10 \mathrm{~mm}$ \\
Zahnbreite & $W$ & $90 \mathrm{~mm}$ \\
Teilkreisdurchmesser & $d_{\mathrm{w}}$ & $260 \mathrm{~mm}$ \\
\hline
\end{tabular}

Die Abmessungen des untersuchten geradverzahnten Zahnrads sind in Tabelle 3.2 zusammengefasst. Dabei wird mit $\imath=0^{\circ}$ ein geradverzahntes Zahnrad eingesetzt, um Gegenlichtaufnahmen des Strahlaufpralls zu ermöglichen.

Tabelle 3.2: Geometrie des untersuchten Zahnrads

\begin{tabular}{lcc}
\hline Parameter & Symbol & Wert \\
\hline Zähnezahl & $Z$ & 65 \\
Eingriffswinkel & $\gamma$ & $25^{\circ}$ \\
Schrägungswinkel & $\imath$ & $0^{\circ}$ \\
Modul & $\mathrm{m}$ & $4 \mathrm{~mm}$ \\
Profilverschiebung & & $+1 \mathrm{~mm}$ \\
Fußradius & & $0,8 \mathrm{~mm}$ \\
Fußkreisdurchmesser & & $252 \mathrm{~mm}$ \\
Wälzkreisdurchmesser & $d_{\mathrm{w}}$ & $260 \mathrm{~mm}$ \\
Kopfkreisdurchmesser & & $270 \mathrm{~mm}$ \\
\hline
\end{tabular}

Das untersuchte Zahnrad wird durch Vorverzahnen und anschließendes Schleifen hergestellt. Aus Gründen, die sich aus der in Kapitel 4 beschriebenen Methode zur Bestimmung des Wärme- 
übergangs ergeben, wird die Titanlegierung TiAl6V4 als Zahnradmaterial eingesetzt. Bei dieser Kombination aus Herstellverfahren und Material sind mittlere Rauheiten der Flanken von etwa $R_{\mathrm{a}}=0,4-0,6 \mu \mathrm{m}$ zu erwarten.

\section{Variationsparameter des Öl-Zahnrad-Wärmeübergangs}

In den meisten Anwendungsfällen in der Luftfahrt sind für Getriebe variable Drehzahlen zu erwarten. Dies gilt sowohl für Hilfsgerätegetriebe in Turbofantriebwerken als auch für Leistungsgetriebe, die in Turboprop- oder Getriebefantriebwerken eingesetzt werden. Auch in der vorliegenden Arbeit wird die Drehzahl $n$ des gekühlten Zahnrads daher als Parameter betrachtet und variiert.

Der Ölvolumenstrom $\dot{V}$ hatte in den Arbeiten von Townsend und Akin (1983), Handschuh (1992) und Schober (1983a) einen deutlichen Einfluss auf die gemessenen Zahnradtemperaturen. Zudem zeigen die kinematische Betrachtung von Akin et al. (1975) sowie die in Abschnitt 3.3 abgeleitete Beschreibung die Abhängigkeit des Strömungsverhaltens vom Ölvolumenstrom auf. Massini et al. (2016) bestätigen die Bedeutung des Geschwindigkeitsverhältnisses von Ölstrahl und Zahnflanke für das Auftreffen des Ölstrahls. Dies legt es nahe, den Ölvolumenstrom ebenfalls als Variationsparameter zu betrachten.

Bisherige Untersuchungen können unterteilt werden in solche, bei denen Öl in radiale Richtung eingespritzt wird, und solche, bei denen das Öl tangential in den Zahnradeingriff eingespritzt wird. Bei tangentialer Einspritzung kann die Düse entweder in Drehrichtung vor oder nach dem Zahnradeingriff angeordnet sein (engl.: into-mesh und out-of-mesh). Die Anordnung der Düse kann durch ihren Einstellwinkel $\alpha$, der im Vorangegangenen definiert wurde, beschrieben werden. Die radiale und tangentiale Anordnung entsprechen dabei den Einstellwinkeln $0^{\circ}$ und $90^{\circ}$. Fondelli et al. (2015), Keller et al. (2017) und Massini et al. (2017) betrachten den Einfluss unterschiedlicher Einstellwinkel numerisch und beobachten deutliche Einflüsse auf das Strömungsverhalten. Dies wird durch die kinematische Betrachtung des Einspritzvorgangs bestätigt. $\alpha$ wird im Rahmen dieser Arbeit als variabler Parameter betrachtet.

Aufgrund der starken Temperaturabhängigkeit der Viskosität der typischerweise in der Luftfahrt verwendeten Öle ist davon auszugehen, dass auch die Temperatur den Öl-Zahnrad-Wärmeübergang beeinflusst. Das Auftreten von laminaren oder turbulenten Ölstrahlen hängt wesentlich von der Reynoldszahl ab, die mit der Viskosität gebildet wird. Die Viskosität bzw. die Temperatur des Öls $\vartheta_{\text {s }}$ wird im Kontext dieser Arbeit ebenfalls als Parameter untersucht und variiert.

Ein Betriebspunkt des betrachteten Systems wird durch vier variable Parameter beschrieben:

- Drehzahl $n$

- Ölvolumenstrom $\dot{V}$

- Düsenwinkel $\alpha$

- Öltemperatur $\vartheta_{\mathrm{s}}$

Der untersuchte Wertebereich der obenstehenden Parameter in dieser Arbeit wird in Tabelle 3.3 zusammengefasst. 
Tabelle 3.3: Wertebereich der Untersuchungen

\begin{tabular}{rc}
\hline Parameter & Bereich \\
\hline$n$ & bis $6000 / \mathrm{min}$ \\
$\dot{V}$ & bis $10,41 / \mathrm{min}$ \\
$\alpha$ & $-45-45^{\circ}$ \\
$\vartheta_{\mathrm{s}}$ & $60,80^{\circ} \mathrm{C}$ \\
\hline
\end{tabular}

\subsection{Düsenströmung}

Die Gestaltung der vorangegangenen Abschnitt beschriebenen Düse und der gewählte Parameterbereich der Versuche führen zum Auftreten verschiedener Strömungsformen des Ölstrahls. Sowohl anliegende als auch abgelöste Ölstrahlen werden beobachtet. Diese unterscheiden sich in ihrem Durchmesser, was zu unterschiedlichen Geschwindigkeiten führt, als auch durch den Turbulenzgrad der Strömung. Um die gemessenen Wärmeübergangsverteilungen sinnvoll auswerten zu können, ist die Unterscheidung zwischen diesen Erscheinungsformen notwendig. Im Folgenden werden die zu erwartenden Strömungsformen und ihre charakteristischen Eigenschaften wiedergegeben.

Die Erzeugung eines Ölstrahls mit ausreichender Geschwindigkeit ist einerseits für die Einspritzkühlung notwendig, um hohe Staudrücke, geringe Grenzschichtdicken und somit hohe Wärmeübergänge sicherzustellen. Andererseits setzt auch die Kinematik des Ölstrahls hohe Strahlgeschwindigkeiten voraus, um ein tiefes Eindringen des Öls in die Zahnlücke zu ermöglichen. Sowohl das Geschwindigkeitsprofil des Strahls, das aus der Düsengeometrie und der eingespritzten Ölmenge resultiert, als auch die Queranströmung durch die Luft, die vom Zahnrad mitbewegt wird, sind dafür entscheidend. Die strömungsmechanischen Phänomene, die hierbei eine Rolle spielen, werden von verschiedenen Autoren eingehend betrachtet und sollen in dieser Arbeit nicht detailliert beleuchtet werden. Eine Übersicht über die Phänomene der Düsenströmung wird unter anderem von Birouk und Lekic (2009) und von Kuensberg Sarre et al. (1999) gegeben. Der Zerfall von querangeströmten Flüssigkeitsstrahlen wird durch Faeth et al. (1995) zusammengefasst.

Schweitzer (1937) beobachtet, dass Flüssigkeitsstrahlen in ruhender Gasumgebung, ähnlich zur Rohrströmung, sowohl in laminarer als auch in turbulenter Form auftreten können. Entscheidend für die Ausbildung der jeweiligen Strömungsform ist dabei die Reynoldszahl des Strahls, definiert als:

$$
\operatorname{Re}_{d}=\frac{\rho u_{\mathrm{s}} d}{\mu}=\frac{u_{\mathrm{s}} d}{v}
$$

In Analogie zur Rohrströmung ist unterhalb einer kritischen Reynoldszahl mit laminarer Strömung des Ölstrahls zu rechnen. Ab der kritischen Reynoldszahl können Störungen in der Düsenströmung ungedämpft anwachsen und zu einer vollturbulenten Düsenströmung führen. Im Fall von Rohrströmungen liegt die kritische Reynoldszahl im Bereich von etwa $\operatorname{Re} \approx 2300$ (Rotta 
(1956)). Liegen, durch geeignete Gestaltung der Düse, nur sehr geringe Störungen vor, kann sich auch oberhalb der kritischen Reynoldszahl ein laminarer Strahl ausbilden. Entscheidende Geometrieeinflüsse sind unter anderem der Einlauf der Düse, die Rauigkeit der Bohrung und die Anwesenheit von Krümmungen und Querschnittsänderungen (Giffen und Muraszew (1953)). Diese beeinflussen die Bedingungen am Auslass der Düse und somit den anschließenden Zerfall des Strahls. Wu et al. (1994) beobachten laminare Strahlen bei $\mathrm{Re} \approx 2 \cdot 10^{4}$ und einer Düsengeometrie mit $\frac{l}{d}=7$. Bei noch kleineren Verhältnissen $\frac{l}{d}<4-6$ wird auch bei $\operatorname{Re} \approx 1 \cdot 10^{6}$ ein laminarer Strahl festgestellt.

Das Strömungsregime in der Düse hat Auswirkungen auf das Geschwindigkeitsprofil an ihrem Austritt und den Durchflussbeiwert, der den notwendigen Öldruck bestimmt. Nach dem Austritt aus der Düse interagiert der Ölstrahl mit der ihn umgebenden Luft. Bei einer ausreichend langen freien Wegstrecke zerfällt der Strahl zu Tropfen. Dabei treten für laminare und turbulente Strahlen unterschiedliche Zerfallsmechanismen auf. Der Zusammenhang zwischen Zerfallslänge $L$ des Strahls und der Austrittsgeschwindigkeit $u$ ist qualitativ in Abbildung 3.2 dargestellt. Bei geringen Austrittsgeschwindigkeiten und laminarer Strömung dominiert der Rayleigh-Zerfall, bei dem die Oberflächenspannung der Flüssigkeit im umgebenden Gas zur Bildung einzelner Tropfen führt (Bereich A und B). Mit zunehmender Austrittsgeschwindigkeit steigt der Abstand zwischen dem Düsenaustritt und dem Beginn des Zerfalls zunächst an, fällt dann jedoch wieder ab.

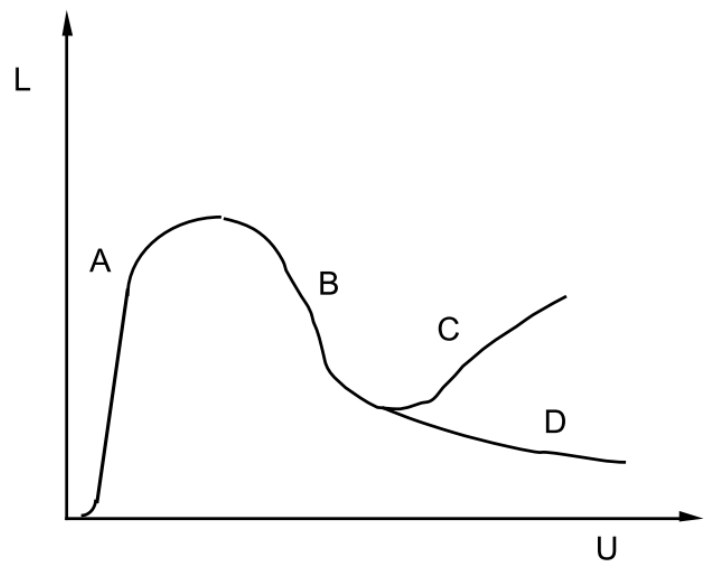

Abbildung 3.2: Verlauf der Zerfallslänge eines Ölstrahls in Abhängigkeit der Strömungsgeschwindigkeit nach Lin und Reitz (1998)

Bei weiterer Erhöhung der Austrittsgeschwindigkeit kann die Zerfallslänge weiter absinken, wenn der Strahl turbulent wird. In diesem Fall führt die Turbulenz zum Auffächern des Strahls unter Verzögerung der mittleren Strahlgeschwindigkeit (Bereich D). Die dabei entstehenden Tropfen sind wesentlich kleiner als der Düsendurchmesser. Ist die Strömung in der Düse zusätzlich abgelöst, etwa durch eine scharfkantige Einschnürung im Düseneinlass (siehe Abbildung 3.3), kann die turbulente Zerfallslänge jedoch auch deutlich ansteigen (Bereich C). 
Anliegende Strömung

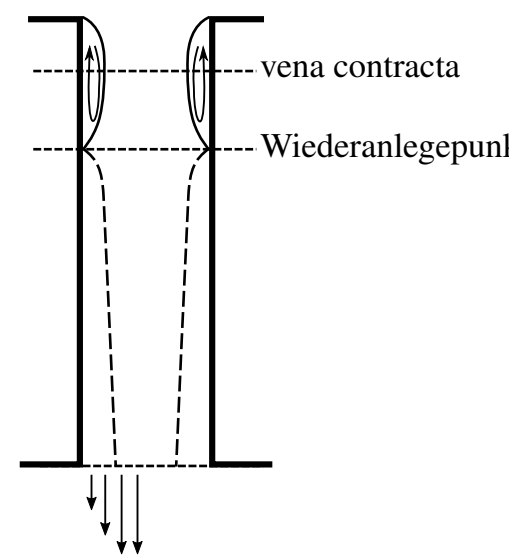

Abgelöste Strömung

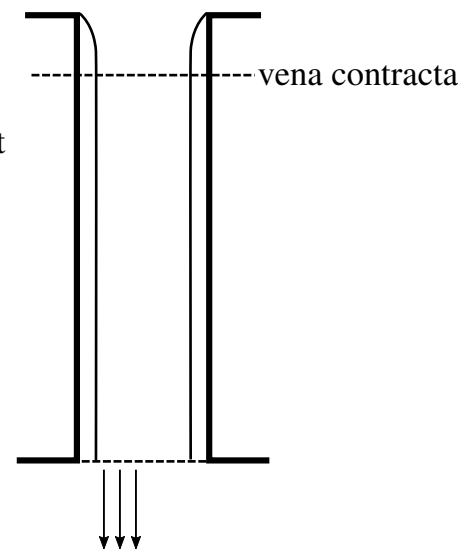

Abbildung 3.3: Anliegende und vollständig abgelöste Düsenströmungen nach von Kuensberg Sarre et al. (1999) 


\subsection{Kinematik des Ölstrahls}

Die Bedeutung der zugeführten Ölmenge für den Wärmehaushalt des Getriebes wird von einigen Autoren diskutiert. In der entwicklungsnahen Fachliteratur werden häufig bestimmte, auf Erfahrungswerten beruhende Ölvolumenströme empfohlen, um ausreichende Kühlung sicherzustellen. So nennen Winter und Michaelis (1984) einen zur Kühlung notwendigen, auf die Zahnbreite bezogenen Mindestölvolumenstrom:

$$
\frac{\dot{V}}{W} \approx 0,08-0,11 / \min
$$

Handschuh (1992) bestimmt die Oberflächentemperaturen an Kegelrädern unter Last und Einspritzschmierung durch einen Fächerstrahl. Die beobachteten Oberflächentemperaturen nehmen bei Erhöhung des Ölvolumenstroms ab. Es zeichnet sich ein asymptotischer Verlauf ab, der Effekt des Ölvolumenstroms ist mäßig. Diese qualitative Beobachtung wird durch Townsend und Akin (1983) für einspritzgekühlte Stirnräder bestätigt.

Eine systematische Methode zur Bestimmung des Wärmeübergangs in Abhängigkeit der geometrischen Parameter und Betriebsgrößen wird von den aufgeführten Quellen nicht dargelegt. Einige Quellen geben Hinweise auf die zu Grunde liegenden Abhängigkeiten. Die Vermutung liegt nahe, dass die kinematischen Beziehungen zwischen bewegter Zahnkontur und eindringendem Ölstrahl einen erheblichen Einfluss auf die Kühlwirkung des Öls haben. Akin et al. (1975) greifen diesen Gedanken auf, indem sie den Einfluss der Eindringtiefe des Ölstrahls in radialer Richtung auf den Wärmeübergang betrachten. Die Autoren stellen ein kinematisches Modell des Einspritzvorgangs auf, das in Abbildung 3.4 dargestellt ist. 


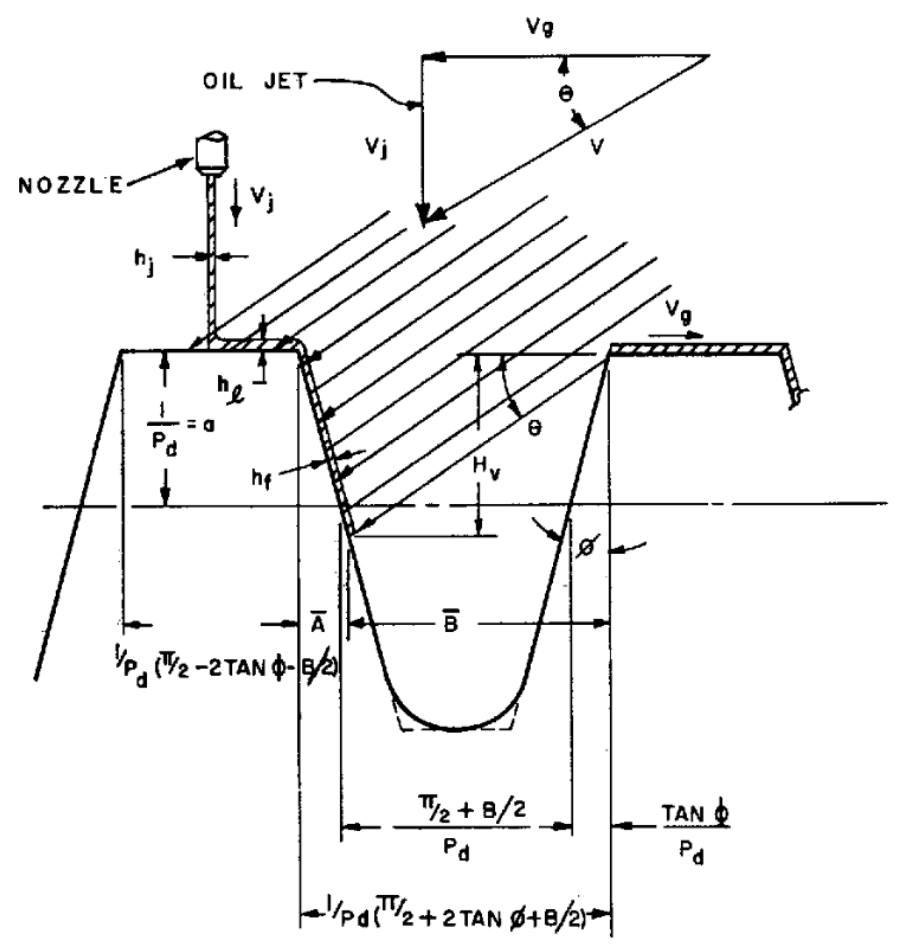

Abbildung 3.4: Kinematisches Ölaufprallmodell nach Akin et al. (1975)

Das Modell von Akin et al. (1975) erlaubt die Berechnung der Eindringtiefe unter Vereinfachung des Einspritzvorgangs. Dazu wird die Rotationsbewegung des Zahnrads als lineare Bewegung modelliert. Es wird weiterhin vorausgesetzt, dass Öl radial eingespritzt wird. Die Geschwindigkeit des Ölstrahls wird als homogen angenommen und abhängig vom Versorgungsdruck unter Annahme einer reibungsfreien Beschleunigung berechnet. Zudem wird der Einfluss der vom Zahnrad mitbewegten Luft, die den Ölstrahl quer anströmt, als gering angesehen und daher nicht berücksichtigt. Anhand der Ölstrahlgeschwindigkeit und Zahngeschwindigkeit bestimmen die Autoren den Eindringwinkel $\beta$, unter dem der Ölstrahl in die Verzahnung eindringt. Townsend und Akin (1983) berechnen anhand des Modells von Akin et al. (1975) die Eindringtiefe des Ölstrahls für die experimentell untersuchten Bedingungen. Für die Betriebsbedingungen, bei denen der Ölstrahl den Zahnfuß erreicht, werden die geringsten Temperaturen und somit die größte Kühlwirkung beobachtet.

\section{Erweiterung des kinematischen Modells zur Berücksichtigung des Düsenwinkels $\alpha$}

Das Modells von Akin et al. (1975) wird im Folgenden erweitert, um die betrachtete Einspritzkühlung zu beschreiben. Dazu wird die Relativgeschwindigkeit des Ölstrahls, $\vec{u}_{\mathrm{r}}$, als Vektorsumme aus der Geschwindigkeit des Zahnrads, $\vec{u}_{\mathrm{z}}$, und der Ausbreitungsgeschwindigkeit des Ölstrahls in Richtung der Düsenachse, $\vec{u}_{\mathrm{s}}$, betrachtet. Um den Einfluss des Düsenwinkels $\alpha$ zu berücksichtigen, wird $\vec{u}_{\mathrm{s}}$ unter dem Winkel $\alpha$ angesetzt. Eine schematische Darstellung dieses Modells ist in Abbildung 3.5 gegeben. 


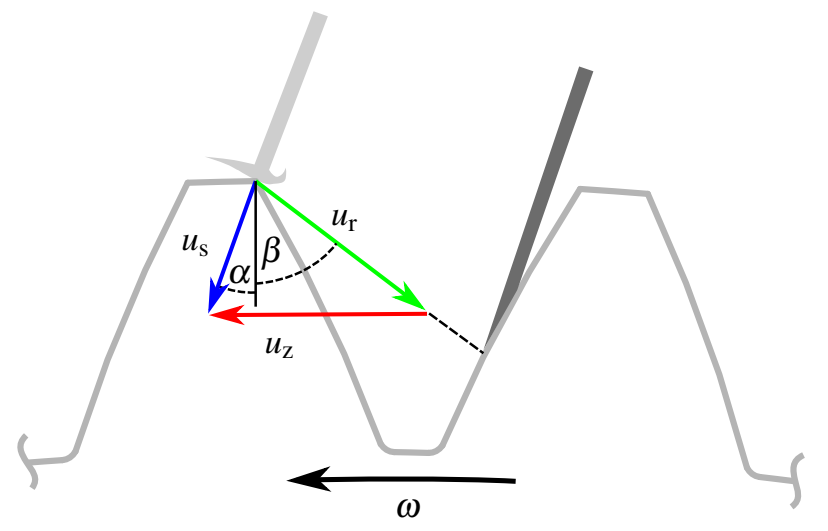

Abbildung 3.5: Kinematik des Ölstrahls unter Berücksichtigung des Düsenwinkels

Zur Interpretation der Ergebnisse dieser Arbeit wird der Winkel $\beta$ aus Abbildung 3.5 verwendet, der in Anlehnung an die Literatur im Folgenden als Aufprallwinkel bezeichnet wird. Zur Veranschaulichung des Wertebereichs von $\beta$ sind in Abbildung 3.6 vier signifikante Aufprallwinkel eingezeichnet. Bei Werten für $\beta$ von unter $28,7^{\circ}$ erreicht der Ölstrahl auch die strömungsabgewandte Flanke. Bei einem Einfall des Ölstrahls mit $\beta=33,8^{\circ}$ erreicht der Ölstrahl die Zahnfußrundung und damit die gesamte strömungszugewandte Zahnflanke. Bei einem Aufprallwinkel von $\beta=60,3^{\circ}$ erreicht der Ölstrahl etwa die Hälfte der Zahnhöhe. Der Strahl triff dabei an seiner Front orthogonal auf die Zahnflanke auf. Ab dem Punkt des orthogonalen Auftreffens und bei größeren Werten für $\beta$ besitzt das Öl keine Geschwindigkeitskomponente in Richtung des Zahnfußes. Nach dem Aufprall ist in diesem Fall nicht mit einem weiteren Eindringen des Öls in Richtung des Zahnfußes zu rechnen.

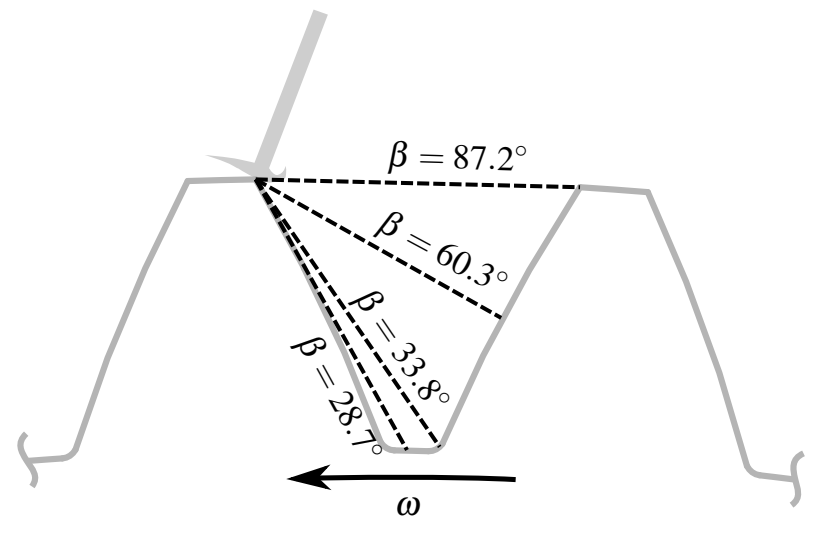

Abbildung 3.6: Diskrete Werte des Aufprallwinkels an vier signifikanten Stellen der Flanke

Um eine vektorielle Betrachtung des Ölaufpralls anstellen zu können, muss die Verteilung der axialen Geschwindigkeit des Ölstrahls als homogen betrachtet werden. Zur Berechnung der axialen Geschwindigkeit wird in der Literatur meist die mittlere Geschwindigkeit in der Düse

$$
u_{\mathrm{s}}=\frac{\dot{V}}{A_{d}}
$$


aus Volumenstrom $\dot{V}$ und Düsenquerschnitt $A_{d}$ herangezogen. Im vorliegenden Fall ist weder bei laminaren noch bei turbulenten Strahlen eine homogene Geschwindigkeitsverteilung zu erwarten. Zusätzlich kann der Strahldurchmesser bei Ablösung der Strömung in der Düse deutlich reduziert sein, wodurch aus Kontinuitätsgründen höhere Strahlgeschwindigkeiten auftreten. Das kinematische Modell liefert unter Verwendung der Geschwindigkeit $u_{\mathrm{s}}$ nur Näherungswerte. Eine genauere Beschreibung erfordert die Charakterisierung der Düsenströmung der eingesetzten Düse, die im Rahmen dieser Arbeit nicht erfolgt. Arbeiten wie die von Townsend und Akin (1983) und die Betrachtung der Ergebnisse in Kapitel 6 zeigen jedoch, dass die vereinfachte Betrachtung zur Beurteilung der auftretenden Wärmeübergangsverteilung bereits ausreichend ist.

Unter den getroffenen Annahmen sind der Aufprallwinkel $\beta$ und der spezifische Auftreffimpuls $\frac{u_{\mathrm{r}}}{u_{\mathrm{z}}}$ nur von den geometrischen Verhältnissen und nicht von absoluten Geschwindigkeiten abhängig. Daher kann zur Vereinfachung der Betrachtung das Geschwindigkeitsverhältnis

$$
\Sigma=\frac{u_{\mathrm{s}}}{u_{\mathrm{z}}}
$$

aus Ölstrahlgeschwindigkeit und Tangentialgeschwindigkeit des Zahnrads gebildet werden. Für den Winkel $\beta$ gilt:

$$
\beta=\operatorname{atan}\left(\frac{u_{\mathrm{r}, \mathrm{t}}}{u_{\mathrm{r}, \mathrm{r}}}\right) .
$$

Im geschilderten Modell ist der Winkel $\alpha$ positiv, wenn die Düse in die Drehrichtung des Zahnrads gerichtet ist. Somit gilt für die Tangentialkomponente der Relativgeschwindigkeit

$$
u_{\mathrm{r}, \mathrm{t}}=u_{\mathrm{z}}-u_{\mathrm{s}, \mathrm{t}}
$$

und somit

$$
\beta=\operatorname{atan}\left(\frac{u_{\mathrm{z}}-u_{\mathrm{s}, \mathrm{t}}}{u_{\mathrm{s}, \mathrm{r}}}\right) .
$$

Durch Bestimmen und Einsetzen der Tangential- und Radialkomponenten der Strahlgeschwindigkeit kann der Term in Gl. 3.8 umgeformt werden zu

$$
\beta=\operatorname{atan}\left(\frac{u_{\mathrm{z}}-\sin (\alpha) \cdot u_{\mathrm{s}}}{\cos (\alpha) \cdot u_{\mathrm{s}}}\right)
$$

Durch Einsetzen des Geschwindigkeitsverhältnisses $\Sigma$ kann $\beta$ bestimmt werden durch

$$
\beta=\operatorname{atan}\left(\frac{\frac{1}{\Sigma}-\sin (\alpha)}{\cos (\alpha)}\right) .
$$

Der Zusammenhang zwischen $\Sigma, \alpha$ und $\beta$ aus Gleichung 3.10 ist in Abbildung 3.7 dargestellt. Bei $\Sigma=1$ führt eine Änderung des Düsenwinkels zu einer linearen Änderung des Aufprallwinkels. 
Für die radiale Einspritzung mit $\alpha=0^{\circ}$ beträgt der Aufprallwinkel $\beta=45^{\circ}$. Bei $\Sigma<1$ existiert ein Minimum des Aufprallwinkels, mit:

$$
\beta_{\min }=\arcsin (\Sigma)
$$

bei dem Düsenwinkel

$$
\alpha_{\beta_{\min }}=90^{\circ}-\arcsin (\Sigma) .
$$

Das Minimum des Aufprallwinkels ist in Abbildung 3.7 als gestrichelte Linie gekennzeichnet. Eine Erhöhung des Düsenwinkels zu höheren Werten führt bei $\alpha>\alpha_{\beta_{\min }}$ zu flacheren Aufprallwinkeln und zusätzlich geringeren Relativgeschwindigkeiten. Daher kann $\alpha_{\beta_{\min }}$ als obere Grenze für den Düsenwinkel gesehen werden, um die Kühlwirkung zu maximieren.

Bei Geschwindigkeitsverhältnissen $\Sigma>1$ existiert diese Grenze nicht und der Aufprallwinkel sinkt monoton mit $\alpha$ und $\frac{\partial^{2} \beta}{\partial \alpha^{2}}<0$.

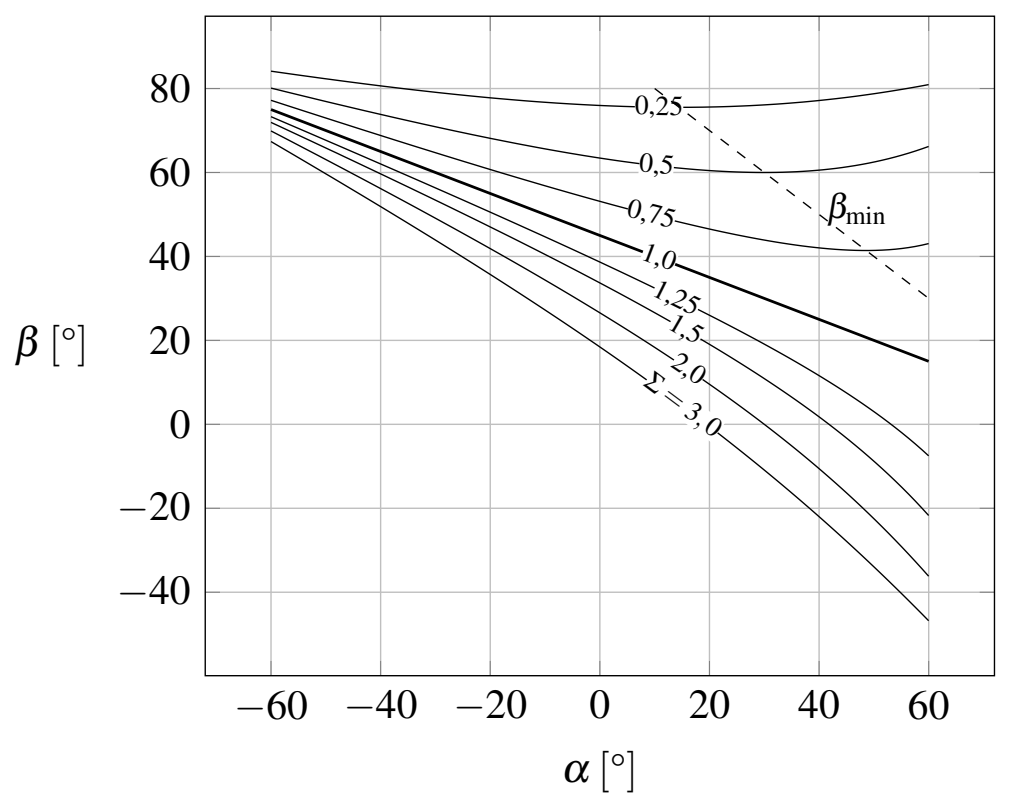

Abbildung 3.7: Einfluss des Düsenwinkels auf den Aufprallwinkel $\beta$ für verschiedene Geschwindigkeitsverhältnisse $\Sigma$

Aus der Übersicht zur Prallkühlung in Abschnitt 2.4 wird deutlich, dass der Impuls des Prallstrahls einen entscheidenden Einfluss auf den Wärmeübergang hat. Auch im vorliegenden Fall kann der relative Impuls herangezogen werden, um das beobachtete Verhalten des Wärmeübergangs zu interpretieren. Analog zur Bestimmung des Aufprallwinkels kann $u_{\mathrm{r}}$ aus der kinematischen Betrachtung abgeleitet werden. Um den Impuls in Abhängigkeit von $\Sigma$ und $\alpha$ zu bestimmen, wird der spezifische Impuls gebildet:

$$
\frac{u_{\mathrm{r}}}{u_{\mathrm{z}}}=\sqrt{1+\Sigma^{2}-2 \Sigma \sin (\alpha)}
$$

Für die Herleitung des Zusammenhangs sei auf den Anhang verwiesen. Aus der Darstellung des spezifischen Impulses aus Gleichung 3.13 in Abbildung 3.8 ist ersichtlich, dass eine Erhöhung 


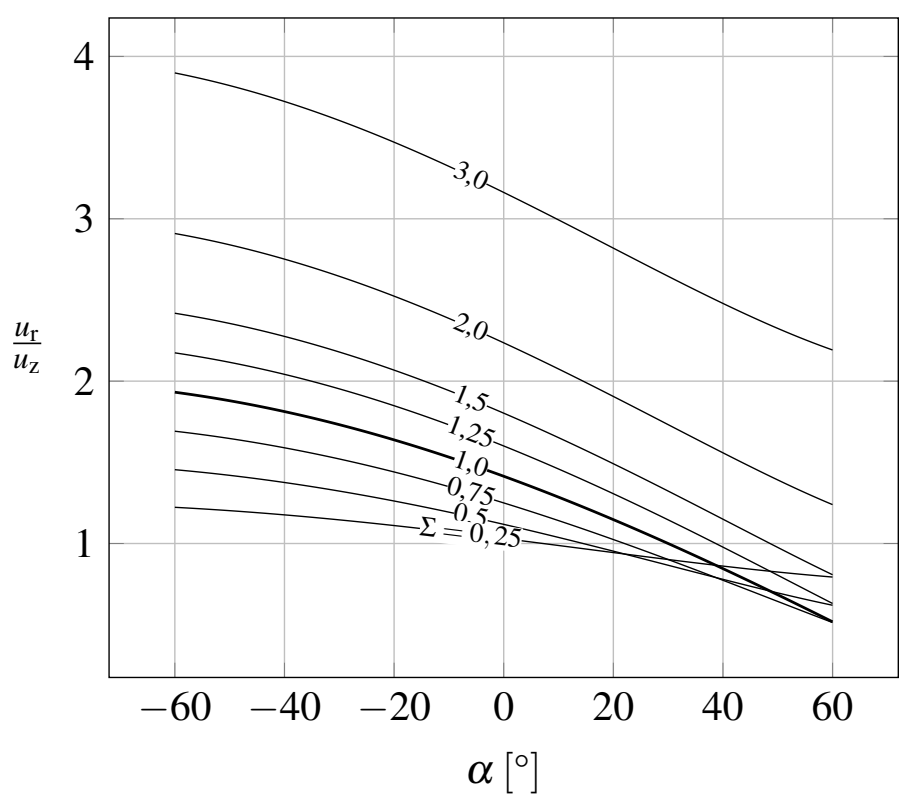

Abbildung 3.8: Einfluss des Düsenwinkels auf den Relativimpuls $\frac{u_{\mathrm{r}}}{u_{\mathrm{z}}}=\sqrt{1+\Sigma^{2}-2 \Sigma \sin (\alpha)}$ für verschiedene Geschwindigkeitsverhältnisse $\Sigma$

des Düsenwinkels stets zu einer Reduzierung der spezifischen Relativgeschwindigkeit führt. Je geringer das Geschwindigkeitsverhältnis $\Sigma$ ist, desto geringer ist der Einfluss des Düsenwinkels auf den Impuls. Dies ist für den Grenzfall $\Sigma \rightarrow 0$ einfach nachvollziehbar, bei dem der Ölstrahl keine Eigengeschwindigkeit und somit stets die Relativgeschwindigkeit $u_{\mathrm{r}}=u_{\mathrm{z}}$ besitzt.

Aus der Betrachtung des Aufprallwinkels wurde deutlich, dass ein positiver Düsenwinkel, also Einspritzung in Richtung der Laufrichtung des Zahnrads, zu einem tieferen Eindringen des Ölstrahls führen kann. Tiefes Eindringen wird in der Literatur mit vergleichsweise hoher Kühlwirkung in Verbindung gebracht und ist somit für die effiziente Nutzung des Kühlmittels vorteilhaft. Die Betrachtung des spezifischen relativen Impulses zeigt jedoch, dass der Impuls des Ölstrahls mit dem Düsenwinkel absinkt. Unter Verwendung der Erkenntnisse zur Prallkühlung kann dies als abträglich für hohe Wärmeübergänge betrachtet werden. Somit liegen zwei gegenläufige Einflüsse vor, die den Öl-Zahnrad-Wärmeübergang beeinflussen. Eine Bewertung der jeweiligen Einflüsse ist durch das vereinfachte Modell nicht möglich und kann erst durch die Interpretation der Ergebnisse in Kapitel 6 erfolgen. 


\section{Experimentelle Methode zur Bestimmung des Öl-Zahnrad-Wärmeübergangs}

In Kapitel 2 wird dargelegt, dass die genaue Kenntnis des Wärmeübergangs an einspritzgekühlten Zahnrädern zur Entwicklung von effizienten hochbelasteten Luftfahrtgetrieben unerlässlich ist. Aus der Analyse der Literatur geht hervor, dass Daten bisheriger Untersuchungen ungenügend sind, da der Wärmeübergang durch Kühlung und die Reibung nicht voneinander getrennt werden können. Die experimentelle Methode, mit der im Rahmen dieser Arbeit diese Einschränkung aufgehoben und erstmals der Öl-Zahnrad-Wärmeübergang ermittelt wird, soll im Folgenden vorgestellt werden. Zunächst wird die Erzeugung des Wärmeübergangs für ein Einzelzahnrad und die numerische Methode zur Auswertung der gemessenen Temperaturen beschrieben. Anschließend wird die experimentelle Umsetzung der Methode geschildert. Abschließend wird die Messgenauigkeit der Methode für die untersuchte Geometrie und Messstellenanordnung statistisch analysiert.

\subsection{Erzeugung des Wärmeübergangs}

In Untersuchungen wie der von Townsend und Akin (1981), Leoni (1991) oder Handschuh (1992) werden experimentelle Daten zum Wärmeübergang an einspritzgekühlten Zahnrädern erhoben. In allen bekannten Fällen wird dabei im Zahnradeingriff Leistung übertragen und Verlustwärme freigesetzt. Durch verschiedene Methoden, z.B. Pyrometrie, Einsatz von Thermoelementen, werden Temperaturen auf der Zahnradoberfläche oder im Zahnradinneren gemessen. Die anschließende Analyse der Temperaturen liefert qualitative Informationen zum Einfluss der verschiedenen Kühlparameter wie Ölvolumenstrom oder Ölstrahlkonfiguration. Die Bilanzierung der Wärmequellen und -senken ist jedoch nicht möglich, da sowohl die entstehenden Verluste als auch die Randbedingungen nicht eindeutig und örtlich aufgelöst bestimmt werden können. Somit kann der Wärmeübergang, der durch die Einspritzkühlung hervorgerufen wird, durch diese Untersuchungen nur als stark fehlerbehaftete und globale Größe quantifiziert werden.

In der vorliegenden Arbeit wird der Wärmeübergang durch Einspritzkühlung isoliert von anderen Wärmeströmen gemessen. Dazu wird im Folgenden eine experimentelle Methode vorgestellt, mit der der zur Messung des Wärmeübergangs erforderliche Wärmestrom nicht durch die Reibungswärme eines Zahnradeingriffs erzeugt wird. Vielmehr wird ein einzelnes hohles Zahnrad eingesetzt, auf dessen Außenmantel heißes Öl gespritzt und dessen Innenkontur durch Prallkühlung mit Luft gekühlt wird. Dadurch stellt sich ein Temperaturfeld über das Zahnrad ein, das von den Wärmeübergängen an Außen- und Innenfläche, $h(\vec{x})$ und $h_{\mathrm{K}}$, sowie der Geometrie und der Wärmeleitfähigkeit $\lambda$ des Zahnradmaterials abhängig ist. Durch die Bestimmung des Temperaturfelds kann die Größe $h(\vec{x})$ bestimmt werden. Im Folgenden soll dargelegt werden, unter welchen Umständen dies möglich ist.

In Abbildung 4.1 ist ein Sektor eines hohlen Zahnrads dargestellt, an dessen in Drehrichtung orientierten Zahnflanken Wärme eingetragen und dessen Innenmantel gekühlt wird. Die Wärme- 
übergangskoeffizienten der Zahnflanken und der Zahnradinnenkontur wurden dabei als homogen angenommen. Die der Drehrichtung abgewandten Flanken, die Zahnspitze und die Zahnfußrundung sowie die Seitenflächen wurden zunächst als adiabate Wände betrachtet. Das vordere Viertel des Sektors ist ausgeschnitten, um den qualitativen thermischen Zustand innerhalb des Festkörpers darzustellen. Der Verlauf der Isothermen ist durch durchgezogene Linien, die Wärmestromlinien sind als Strichlinien dargestellt.

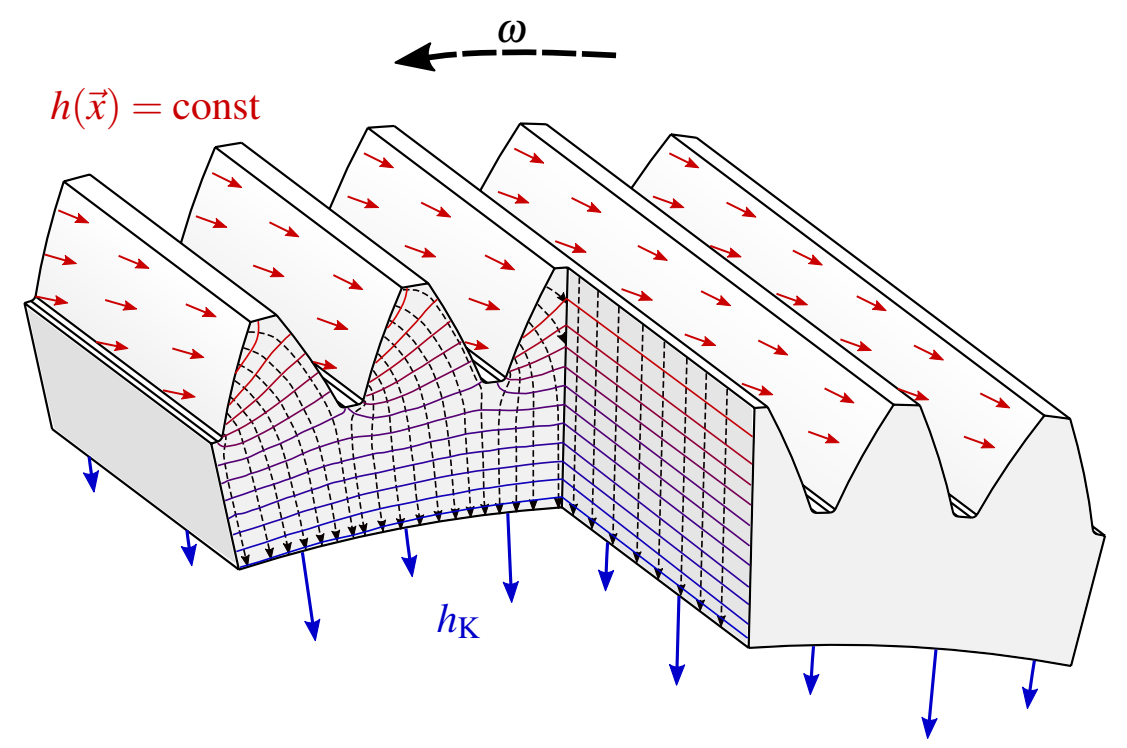

Abbildung 4.1: Qualitative Temperaturverteilung eines Zahnradsektors mit vereinfachten thermischen Randbedingungen

Trotz der angenommenen homogenen Randbedingungen stellt sich im Festkörper ein nicht triviales Temperaturfeld ein. Das Temperaturfeld ist abhängig von den Randbedingungen, der Geometrie des Zahnrads und dem gewählten Material des Festkörpers. Für die Wärmeleitung im Prüfkörper gilt allgemein:

$$
\frac{\partial}{\partial t} \vartheta(\vec{x}, t)-a \Delta \vartheta(\vec{x}, t)=q_{\mathrm{V}}(\vec{x}, t)
$$

mit der Temperatur $\vartheta$, der Temperaturleitfähigkeit $a$ und der volumetrischen Wärmestromdichte $q_{\mathrm{V}}$ an der Stelle $\vec{x}$ zum Zeitpunkt $t$. Die Wärmestromdichte ist in Gleichung 4.1 auf die spezifische Wärmekapazität bezogen. In den vorliegend geschilderten Experimenten werden stets stationäre Temperaturzustände des Versuchszahnrads betrachtet, somit gilt:

$$
\frac{\partial}{\partial t} \vartheta(\vec{x}, t)=0
$$

Zusätzlich gilt für die im Rahmen dieser Arbeit betrachteten Experimente Quellen- und Senkenfreiheit, da im Zahnrad keine Wärme freigesetzt wird. Es gilt:

$$
q_{\mathrm{V}}(\vec{x})=0
$$

Dadurch wird Gleichung $4.1 \mathrm{zu}$ :

$$
-a \Delta \vartheta(\vec{x})=0
$$


Gleichung 4.4 ist eine elliptische partielle Differentialgleichung zweiter Ordnung, die LaplaceGleichung genannt wird. Durch die Quellen- und Senkenfreiheit, die im vorliegenden Fall stets gilt, ist die Laplace-Gleichung eindeutig lösbar, sofern ihre Randwerte bekannt sind und die Temperaturleitfähigkeit als konstant betrachtet werden kann. Dies bedeutet, dass die Kenntnis der Temperaturverteilung am Rand des Gebiets, also der Festkörperoberfläche, ausreicht, um die Verteilung der Wärmestromdichte im Gebiet zu bestimmen. Die Ausnutzung dieses Zusammenhangs ist der Kerngedanke der beschriebenen Methode.

Durch die oben beschriebenen Zusammenhänge kann der Wärmestrom im Zahnrad eindeutig durch Messung der Oberflächentemperatur des Zahnrads bestimmt werden. Da an der Oberfläche des Zahnrads nur Wärmeleitung auftritt und keine Wärme freigesetzt wird muss gelten:

$$
\dot{q}_{\mathrm{konv}}(\vec{x})\left\|_{\mathrm{O}}=\dot{q}_{\mathrm{kond}}(\vec{x})\right\|_{\mathrm{O}}
$$

Der Wärmestrom an das Fluid $\dot{q}_{\text {konv }}(\vec{x})$ entspricht demnach dem Wärmestrom an der Oberfläche des Festkörpers $\dot{q}_{\text {kond }}(\vec{x})$. Der Wärmeübergangskoeffizient kann bestimmt werden als:

$$
h(\vec{x})=\frac{\dot{q}_{\mathrm{konv}}(\vec{x})}{\vartheta_{\mathrm{s}}-\vartheta(\vec{x})}
$$

mit der Temperatur des Öls, $\vartheta_{\mathrm{s}}$, oder in dimensionsloser Form als

$$
\mathrm{Nu}(\vec{x})=\frac{h(\vec{x}) m}{\lambda}
$$

mit der Wärmeleitfähigkeit des Öls, $\lambda$, und der charakteristischen Länge, für die hier der Modul des Zahnrads, $m$, eingesetzt wird.

Im Vorangegangenen wird stets davon ausgegangen, dass ein Temperaturgradient vorliegt, der zu einem Wärmeübergang vom eingespritzten Öl in das Zahnrad führt. In der Anwendung in einem Höchstleistungsgetriebe ist eine entgegengesetzte Wärmestromrichtung zu erwarten. Die Umkehrung der Wärmestromrichtung ist aufgrund der experimentellen Umsetzung im Rahmen dieser Arbeit erforderlich, wie in Kapitel 5 erläutert wird. Für die geschilderte Berechnung des Wärmestroms ist die Richtung des Wärmestroms unerheblich. Aufgrund der hervorgerufenen Temperaturänderung im Öl beeinflusst die Richtung des Wärmestroms jedoch die Strömungsvorgänge durch Prallkühlung. Im Fall von Fluiden, deren Viskosität temperaturunabhängig ist, ist der Wärmeübergangskoeffizient temperaturunabhängig, wodurch die Richtung des Wärmeübergangs keine Rolle spielt. Diese Annahme kann für das verwendete Öl nicht getroffen werden. Jedoch wird durch die Auslegung des Versuchszahnrads im Folgenden sichergestellt, dass nur vergleichsweise geringe Temperaturänderungen auftreten und so die Übertragbarkeit der Ergebnisse auf den Anwendungsfall gewahrt bleibt.

Zur experimentellen Bestimmung des Öl-Zahnrad-Wärmeübergangs ist ein Versuchszahnrad erforderlich, dessen Temperaturen im Versuchsbetrieb unter definierten Betriebsbedingungen gemessen werden. Die Geometrie und der Wärmeleitwert des eingesetzten Materials müssen bekannt sein. Sind diese Bedingungen erfüllt, kann der Wärmeübergang durch die Lösung von Gleichung 4.4 bestimmt werden. Aufgrund der Geometrie des Zahnrads, die sich einer analytischen Beschreibung entzieht, muss die Lösung numerisch bestimmt werden. Die Beschreibung 
des Lösungsverfahrens erfolgt im folgenden Kapitel. Die Geometrie des Versuchszahnrads ist in Kapitel 4.4 beschrieben. Auf den Prüfstand, mit dem der Zahnradwärmeübergang unter Einfluss variabler Betriebsbedingungen untersucht wird, wird in Kapitel 5 eingegangen.

\subsection{Modellvereinfachung zur Erhöhung der Auflösung}

In vielen Wärmeübergangsuntersuchungen werden hochaufgelöste flächige Temperaturmessungen durch Thermographie durchgeführt, um die Temperaturverteilung von Oberflächen und somit die thermischen Randbedingungen zu bestimmen (u.a. Kneer (2017), Ochs (2011), Pütz (2018)). Die örtlich hoch aufgelöste Erfassung der Oberflächentemperaturen des Zahnrads ist jedoch nicht möglich. Im vorliegenden Fall verhindern das beteiligte Öl, die hohen Oberflächengeschwindigkeiten und die eingeschränkte optische Zugänglichkeit den Einsatz von Thermographie. Die verfügbaren Öle weisen bereits ab geringen Filmdicken Emissionsgrade von etwa $\varepsilon=1$ auf, weshalb eine infrarotthermographische Messung der Temperatur an den unter dem Ölfilm befindlichen Oberflächen verhindert wird. Zudem ist bei hohen Drehzahlen Öl in der Form von Tropfen und Nebel in der Luft vorhanden, wodurch keine sinnvolle optische Betrachtung des Vorgangs mehr möglich ist.

Für die Bestimmung der Oberflächentemperaturen in dieser Arbeit werden daher Temperaturen durch den Einsatz von Thermoelementen auf dem rotierenden Zahnrad und drahtloser Datenübertragung gemessen. Optische Zugänglichkeit und zeitlich hochaufgelöste Messungen sind hierfür nicht erforderlich. Allerdings ist die Anzahl an Temperaturmesspunkten durch die gegenwärtig verfügbaren Telemetriesysteme eingeschränkt. Um dennoch örtliche Effekte auflösen und beobachten zu können, wird diesem Umstand durch Ausnutzung von Symmetrie- und Periodizitätseigenschaften sowie zielgerichtete Gestaltung des Versuchszahnrads Rechnung getragen. Dazu werden folgende Modellvereinfachungen getroffen:

- Periodizität: Nahezu alle Thermalmodelle in der Literatur nutzen die geometrische Periodizität von Zahnrädern, um den Modellierungsaufwand auf einen Einzelzahn zu beschränken. Diese Eigenschaft kann auch messtechnisch ausgenutzt werden. Die Schnittebenen eines periodischen Elements können in einfachsten Fällen als adiabate Wand modelliert werden. Wärmeströme zwischen zwei Zahnelementen sind jedoch grundsätzlich möglich und können durch die Implementierung periodischer Ränder berücksichtigt werden. Dabei gilt an der Schnittebene:

$$
\dot{q}_{\mathrm{A}}(\vec{x})=-\dot{q}_{\mathrm{B}}(\vec{x})
$$

sowie

$$
\vartheta_{\mathrm{A}}(\vec{x})=\vartheta_{\mathrm{B}}(\vec{x})
$$

Anschaulich bedeutet die Forderung aus Gleichung 4.8, dass an korrespondierenden Punkten $\vec{x}$ auf den periodischen Rändern A und B die gleichen Wärmeströme ein- und ausfließen sowie gleiche Temperaturen herrschen (Gleichung 4.9). Im Fall des geradverzahnten Zahnrads bieten sich Schnittebenen konstanten Drehwinkels an (siehe Abbildung 4.2). 
- Symmetrie: Die in dieser Untersuchung eingesetzte Ölbrause weist vier identische Einzeldüsen auf, die in regelmäßigen Abständen angeordnet sind. Aufgrund dieser Anordnung sind in axialer Richtung periodische Wärmeübergänge zu erwarten. Das Zahnelement kann daher zunächst zu einem Viertel mit periodischen Rändern vereinfacht werden. Durch die Annahme, dass der Wärmeübergang innerhalb eines Viertelzahns zudem symmetrisch ist (siehe Strichlinie in Abbildung 4.2), werden die periodischen Ränder zu Symmetrieebenen. Symmetrieebenen des Wärmeübergangs lassen sich numerisch als adiabate Wände abbilden.

- Biotzahl: Das Verhältnis aus Wärmeübergangskoeffizient und Wärmeleitfähigkeit des Zahnrads ist entscheidend für die Temperaturverteilung innerhalb des Zahnrads. Dabei sind sowohl zu hohe als auch zu niedrige Verhältnisse ungünstig, da sie die Messgenauigkeit negativ beeinträchtigen. Durch geeignete Wahl des Zahnradmaterials, auf die in Abschnitt 4.4 eingegangen wird, kann sichergestellt werden, dass trotz weniger diskreter Messstellen Wärmeübergänge zwischen den Messpunkten berücksichtigt werden.

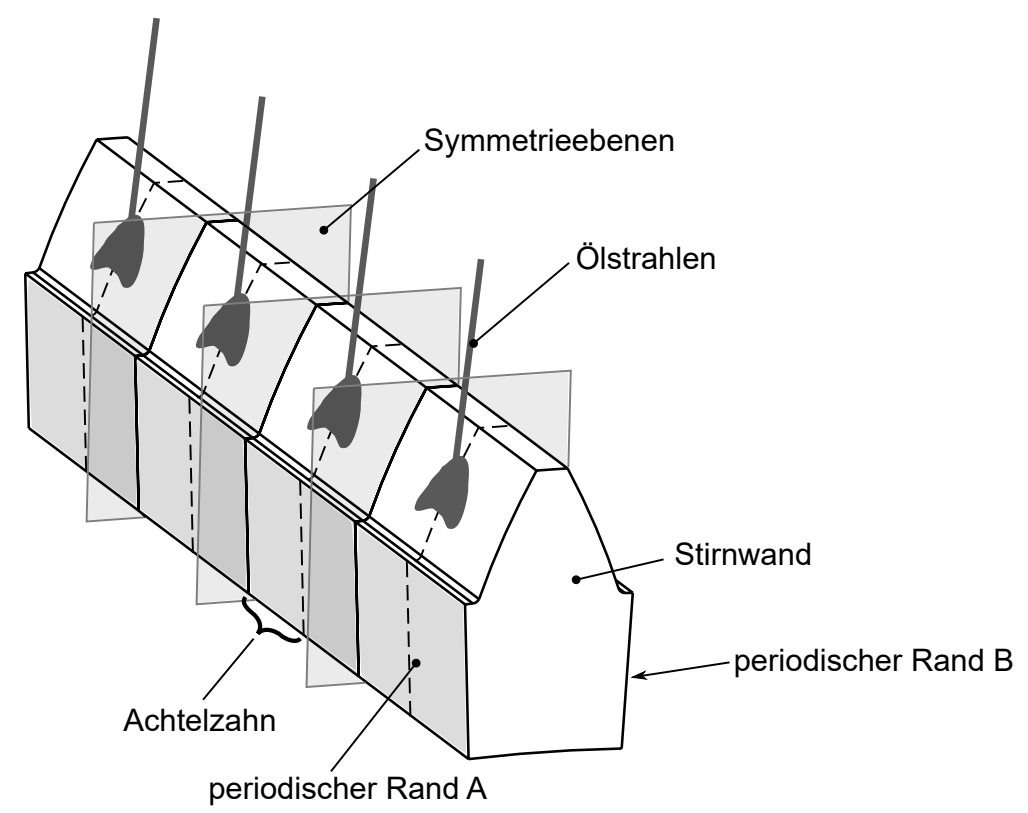

Abbildung 4.2: Vereinfachung des Zahnrads als Viertelzahn - Symmetrieebenen und periodische Ränder

Durch die vorangegangen Vereinfachungen ist es möglich, den Zahnradwärmeübergang durch die Messung diskreter Oberflächentemperaturen auf einem Achtelzahn zu bestimmen. Dadurch können die Messpunkte, die aus technischen Gründen in ihrer Anzahl beschränkt sind, dichter verteilt werden. Der Wärmeübergang an den Stirnwänden des Zahnrads blieb bisher unberücksichtigt. Anhand von Abschätzungen durch Korrelationen lässt sich zeigen, dass das Niveau des Wärmeübergangs der Stirnseiten weit unterhalb des erwarteten Wärmeübergangs durch Ölkühlung liegt und somit nur einen Einfluss im Randbereich des Zahnrads haben wird. Dies entspricht der Beobachtung von Patir und Cheng (1979). Um den Einfluss der Seitenwände auf die gemessenen Wärmeströme gering zu halten, wird im vorliegenden Fall einer der innenliegenden 
Achtelzähne mit Thermoelementen instrumentiert. Um die Annahme der Symmetrie innerhalb des Zahnviertels experimentell überprüfen zu können, wird der benachbarte Achtelzahn ebenfalls mit Messpunkten versehen. Auf die Verteilung der Messpunkte wird in Abschnitt 4.4 genauer eingegangen.

\subsection{Iterative Berechnung des Wärmeübergangs}

Das in Abschnitt 4.1 beschriebene Randwertproblem kann dann gelöst werden, wenn die Randbedingungen vollständig bestimmt sind. Dies setzt voraus, dass die Temperatur jeden Punktes an der Berandung des Festkörpers, an dem keine Neumann-Randbedingung gilt, bekannt ist. Die Temperaturen an der Oberfläche des Zahnrads werden jedoch im Rahmen dieser Arbeit an diskreten Punkten bestimmt. Die Temperaturverteilung zwischen diesen Punkten ist zunächst nicht bekannt. Aus Abbildung 4.1 wird deutlich, dass die Temperaturverteilungen, die sich über das Zahnrad einstellen, aufgrund der Geometrie des Zahnrads komplex sind. Wird diese Temperaturverteilung durch lineare Interpolation angenähert und das Randwertproblem gelöst, ergeben sich unphysikalische Lösungen des Wärmeübergangs. Dies liegt daran, dass die Temperaturverteilung im Fall der linearen Interpolation an den Stützstellen nicht stetig differenzierbar ist. Aber auch die Splineinterpolation ,zwingt” die Finite-Elemente-Methode zu unphysikalischen Lösungen, da ein stückweise polynomieller Temperaturverlauf nicht den sich in der Realität einstellenden Verläufen entspricht.

Physikalisch sinnvolle Lösungen können bestimmt werden, indem das Randwertproblem iterativ gelöst wird. Um die Interpolation von Temperaturwerten zu vermeiden, werden anstatt von Temperaturwerten Wärmeübergangskoeffizienten als Randbedingung verwendet. Das gemischte Randwertproblem wird so in ein Neumann-Randwertproblem überführt, dessen Lösung ebenfalls eindeutig ist. Da nun $h(\vec{x})$, der a priori nicht bekannt ist, sowohl Randbedingung als auch Lösung des Wärmeleitungsproblems ist, muss zur iterativen Lösung ein Gütekriterium und eine Iterationsvorschrift gefunden werden. Die gesuchte Lösung erfüllt die Bedingung

$$
\vartheta_{i}^{n}=\vartheta_{i}^{M}
$$

Anschaulich bedeutet die Forderung aus Gleichung 4.10, dass die Temperaturen der numerischen Lösung $\vartheta_{i}^{n}$ den gemessenen Temperaturen $\vartheta_{i}^{M}$ an der Festkörperoberfläche entsprechen sollen. Daraus lässt sich unmittelbar das Gütekriterium der Iteration ableiten:

$$
\Delta \vartheta_{i}^{n}=\vartheta_{i}^{n}-\vartheta_{i}^{M}
$$

In der Arbeit von Stripf (2007) wird zur Lösung folgender Iterationsansatz vorgestellt:

$$
h_{i}^{n+1}=h_{i}^{n} \cdot \frac{\vartheta_{\mathrm{s}}-\vartheta_{i}^{n}}{\vartheta_{\mathrm{s}}-\vartheta_{i}^{n}-\Delta \vartheta_{i}^{n}}
$$

Voruntersuchungen haben gezeigt, dass der Ansatz nach Gleichung 4.12 im vorliegenden Fall zu unbefriedigendem Konvergenzverhalten führt. In den Wärmeübergangsmessungen von Stripf (2007) sind die Temperaturmesspunkte vergleichsweise dicht verteilt, der Verlauf der 
Wärmeübergangskoeffizienten unterliegt geringen lokalen Schwankungen. Im vorliegenden Fall können jedoch unter bestimmten Bedingungen örtlich sehr begrenzte hohe Wärmeübergänge auftreten. Daher wird folgender Iterationsansatz abgeleitet:

$$
h_{i}^{n+1}=h_{i}^{n}+\widetilde{h}^{n} \frac{\Delta \vartheta_{i}^{n}}{\vartheta_{\mathrm{s}}-\widetilde{\vartheta}^{n}}
$$

mit dem arithmetischen Mittel der Wärmeübergangskoeffizienten

$$
\widetilde{h}^{n}=\frac{\sum_{i=1}^{z} h_{i}^{n}}{z}
$$

und der Temperaturen

$$
\widetilde{\vartheta}^{n}=\frac{\sum_{i=1}^{z} \vartheta_{i}^{n}}{z}
$$

mit der Anzahl der Messpunkte $z$.

Die Implementierung der beschriebenen iterativen Berechnung der Wärmeübergangskoeffizienten ist in Abbildung 4.3 dargestellt. Zur Initialisierung der Berechnung werden Startwerte $h_{i}^{0}$ an den Messpunkten vorgegeben. Der Wärmeübergang an der Zahnradaußenkontur wird auf Grundlage der Startwerte $h_{i}^{0}$ interpoliert. Unter Verwendung dieser Startverteilung des Wärmeübergangs, der gemessenen Temperaturen am Innenradius des Zahnrads, der Zahnradgeometrie sowie der Wärmeleitfähigkeit des Zahnrads wird der Wärmetransport im Zahnrad simuliert. Aus der berechneten Temperaturverteilung werden die Temperaturen an den Messpunkten, $\vartheta_{i}^{n}$, extrahiert. Durch Subtraktion mit den gemessenen Messpunkttemperaturen $\vartheta_{i}^{M}$ kann die Temperaturabweichung $\Delta \vartheta_{i}^{n}$ bestimmt werden. Mit der Iterationsvorschrift (Gleichung 4.13) können anschließend korrigierte Wärmeströme berechnet werden. Bei Unterschreiten einer Schwelle für $\Delta \vartheta_{i}^{n}$ von $0,25 \mathrm{~K}$ für alle $i$ werden die zuletzt verwendeten Wärmeübergangskoeffizienten $h_{i}^{n}$ und die daraus resultierende, interpolierte Wärmeübergangsverteilung als Berechnungsergebnis verwendet. 


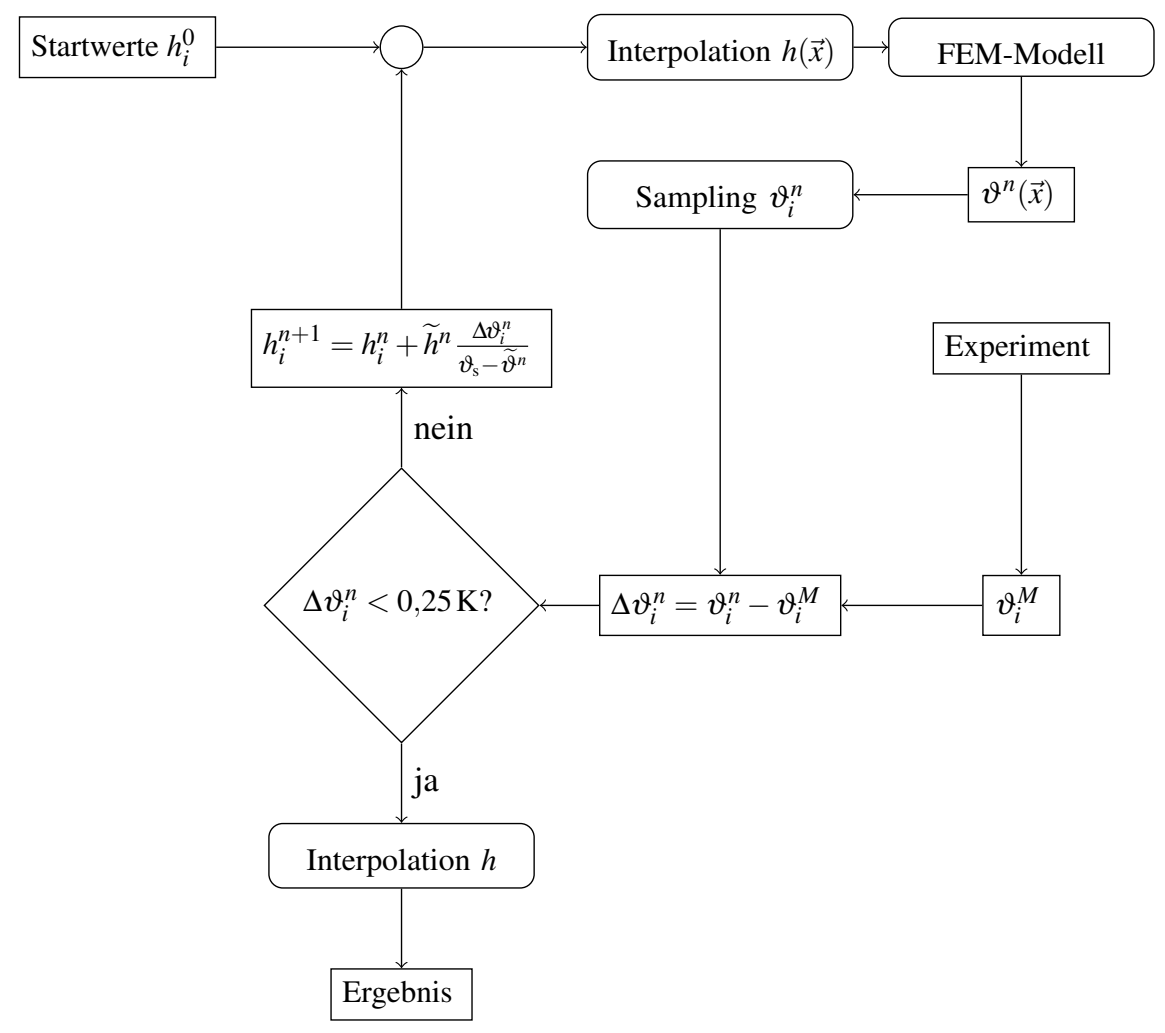

Abbildung 4.3: Schematische Darstellung der iterativen Berechnung des Wärmeübergangs aus diskreten Temperaturmesswerten

\subsection{Experimentelle Umsetzung}

Zur experimentellen Umsetzung der im Vorangegangenen beschriebenen Methode muss ein Versuchszahnrad mit diskreten Messpunkten der Temperatur definiert werden. Die Gestaltung des Versuchszahnrads, das im Rahmen dieser Arbeit eingesetzt wird, sowie die Instrumentierung dieses Zahnrads sollen im Folgenden geschildert werden.

\subsubsection{Optimierte Gestaltung eines Versuchszahnrads}

Die Wahl des Prüfkörpermaterials hat maßgeblichen Einfluss auf die Messgenauigkeit des Wärmeübergangskoeffizienten. Die Außenkontur des Prüfkörpers ist durch die Definition der Zahnradgeometrie und der Düsenanordnung in Kapitel 3 bestimmt. Die Dicke des Zahnradmantels kann jedoch geeignet gewählt werden, um günstige Voraussetzungen für eine möglichst geringe Messunsicherheit zu schaffen. Dabei muss ein Kompromiss getroffen werden, um mehrere Anforderungen zu erfüllen. Die Viskosität des verwendeten Öls ist stark temperaturabhängig. Eine zu große Abkühlung des Öls durch den Wärmeübergang am Zahnrad ist einerseits zu vermeiden, um die Strömungsvorgänge nur wenig zu beeinflussen. Die Temperaturdifferenz zwischen Öl und Zahnradoberfläche geht in den Nenner des Wärmeübergangskoeffizienten ein. Daher sind andererseits Temperaturdifferenzen von mehreren Kelvin notwendig, um den Einfluss der Unsicherheit der Temperaturmessung gering zu halten. Zudem sind bei ungünstiger Wahl des 
Materials lokale Wärmeübergänge nicht messbar. Dies gilt sowohl für Materialien zu geringer als auch zu hoher Wärmeleitfähigkeit.

Für die anschauliche Betrachtung des Zusammenhangs kann die Biotzahl herangezogen werden, definiert als:

$$
\mathrm{Bi}=\frac{h L_{\mathrm{Bi}}}{\lambda}
$$

mit dem Wärmeübergangskoeffizienten $h$, der charakteristischen Länge $L_{\mathrm{Bi}}$ und der Wärmeleitfähigkeit $\lambda$. Für Werkstoffe geringer Wärmeleitfähigkeit nähern sich die Oberflächentemperaturen des Prüfkörpers für Bi $\rightarrow \infty$ der Temperatur der Umgebung bzw. des Öls. Damit werden die gemessenen Temperaturen für hohe Biotzahlen unabhängig vom Wärmeübergang, der Einfluss des stets vorhanden Messfehlers steigt gegen unendlich. Im entgegengesetzten Fall von Werkstoffen sehr hoher Wärmeleitfähigkeit wird das Temperaturfeld des Prüfkörpers für $\mathrm{Bi} \rightarrow 0$ an jeder Stelle den gleichen Wert annehmen, da für $\lambda \rightarrow \infty$ auch kleinste Temperaturgradienten zu einem unendlich hohen Ausgleichswärmestrom führen würden. Auch in diesem Fall kann der lokale Wärmeübergang nicht sinnvoll gemessen werden. Jacobsen (1987) und Glahn (1995) kommen aufgrund ähnlicher Überlegungen für den Wärmeübergang in Labyrinthdichtungen und Lagerkammern zu dem Schluss, dass die Wärmeleitfähigkeit des Prüfkörpers und seine Dimensionierung so gewählt werden muss, dass $\mathrm{Bi} \approx 1$ gilt.

Im vorliegenden Fall kann für $L_{\mathrm{Bi}}$ der Abstand der Messpunkte eingesetzt werden, deren Anordnung im folgenden Abschnitt geschildert wird und etwa $4 \mathrm{~mm}$ beträgt. Die Forderung nach $\mathrm{Bi}=1$ erfordert die Kenntnis des Wärmeübergangs an der Oberfläche des Zahnrads. Dieser ist im Vorfeld nicht bekannt, kann jedoch durch die Korrelation von DeWinter und Blok (1974) sowie aufgrund der in Abschnitt 2.4 geschilderten Untersuchungen des Wärmeübergangs durch Prallkühlung abgeschätzt werden. Daraus resultieren abgeschätzte mittlere Wärmeübergänge in der Größenordnung von $1000 \mathrm{~W} /\left(\mathrm{m}^{2} \mathrm{~K}\right)$. Im vorliegenden Fall wurde die Titanlegierung TiAl6V4 als Material gewählt, die mit $\lambda=7 \mathrm{~W} /(\mathrm{mK})$ und $\mathrm{Bi}=0,57$ die Forderung erfüllt. Durch die sehr geringe Temperaturabhängigkeit der Wärmeleitfähigkeit von TiAl6V4 ist zudem die Gültigkeit von Gleichung 4.4 sichergestellt.

Um die Abhängigkeit der mittleren Temperatur von der Wärmeleitfähigkeit des Versuchszahnrads und dem Wärmeübergangskoeffizienten darzustellen, kann der Einzelzahn als eindimensional betrachtet werden (siehe Abbildung 4.4). Mit der Fourier'schen Wärmeleitungsgleichung, dem Wärmeübergang durch Kühlung am Innenradius des Zahnrads und dem abgeschätzten ÖlZahnrad-Wärmeübergang ergibt sich die Temperatur der Zahnaußenfläche:

$$
\vartheta_{\mathrm{E}}=\vartheta_{\mathrm{s}}-\frac{\vartheta_{\mathrm{s}}-\vartheta_{\mathrm{K}}}{h \frac{L}{\lambda}+\frac{h}{h_{\mathrm{K}}}+1},
$$

mit der Öltemperatur $\vartheta_{\mathrm{s}}$, der Temperatur der Kühlluft und dem Wärmeübergangskoeffizienten auf der Innenseite $\vartheta_{\mathrm{K}}$ und $h_{\mathrm{K}}$, der Dicke des eindimensionalen Vergleichskörpers $L$ sowie dem Wärmeübergangskoeffizienten durch Einspritzkühlung, $h$.

Zur Kühlung der Innenseite des Zahnrads wird ein Runddüsenfeld eingesetzt, das auf dem Außenmantel eines Zylinders angeordnet ist. Unter Verwendung der umfangreichen Literatur zur Berechnung des Wärmeübergangskoeffizienten für Runddüsenfelder kann der Wärmeübergang 
auf der Innenseite des Zahnrads abgeschätzt werden und beträgt etwa $350 \mathrm{~W} /\left(\mathrm{m}^{2} \mathrm{~K}\right.$ ) (VDI (2013)).

Die Oberflächentemperatur nach Gleichung 4.17 ist in Abbildung 4.5 für verschiedene Prüfkörperdicken dargestellt. Dabei wurde eine Öltemperatur von $\vartheta_{\mathrm{s}}=80^{\circ} \mathrm{C}$ und Kühllufttemperatur von $\vartheta_{\mathrm{K}}=20^{\circ} \mathrm{C}$ angenommen. Bei geringen Dicken des Prüfkörpers sind die Temperaturdifferenzen zwischen einspritzgekühlter und luftgekühlter Oberfläche gering, die Oberflächentemperatur ist niedrig. Dadurch sind größere Viskositätsänderungen des Öls und somit geänderte Strömungsvorgänge zu erwarten. Bei einer Prüfkörperdicke von $50 \mathrm{~mm}$ ist die Oberflächentemperatur im erwarteten Bereich wesentlich schwächer abhängig von $h$, was die Messunsicherheit erhöht (siehe rot markierter Bereich in Abbildung 4.5). Aus diesen und aus konstruktiven Gründen wurde der Innendurchmesser des hohlen Zahnrads zu $d_{\mathrm{i}}=230 \mathrm{~mm}$ gewählt, was einer Prüfkörperdicke von $15 \mathrm{~mm}$ entspricht.

\subsubsection{Instrumentierung des Versuchszahnrads}

Um die Anordnung der Messpunkte und die Ergebnisse dieser Arbeit anschaulich darzustellen, wird ein Koordinatensystem eingeführt, das die Zahnflanken beschreibt. Die Anordnung der Koordinaten $s$ und $y$, mit denen die dreidimensionale prismatische Oberfläche des Zahnrads in eine zweidimensionale Darstellung überführt werden kann, sind in Abbildung 4.6 dargestellt. Die Koordinate $s$ verläuft entlang der Zahnkontur und hat ihren Ursprung an der Mitte des Zahnfußes. Die geometrische Symmetrieebene zwischen zwei Zähnen, die auch den periodischen Rand darstellt, verläuft durch $s=0$. Das Ende des Koordinatenbereiches liegt in der Mitte des gegenüberliegenden Zahnfußes bei $s=s_{\max }$. Damit kann die dimensionslose Koordinate

$$
s^{+}=\frac{s}{s_{\max }}
$$

eingeführt werden. Die axiale Koordinate $y$ verläuft von der vorderseitigen Stirnwand bei $y=0$ bis zur rückseitigen Stirnwand bei $y=W$. Analog zu $s^{+}$gilt für die dimensionslose axiale Koordinate

$$
y^{+}=\frac{y}{W} .
$$

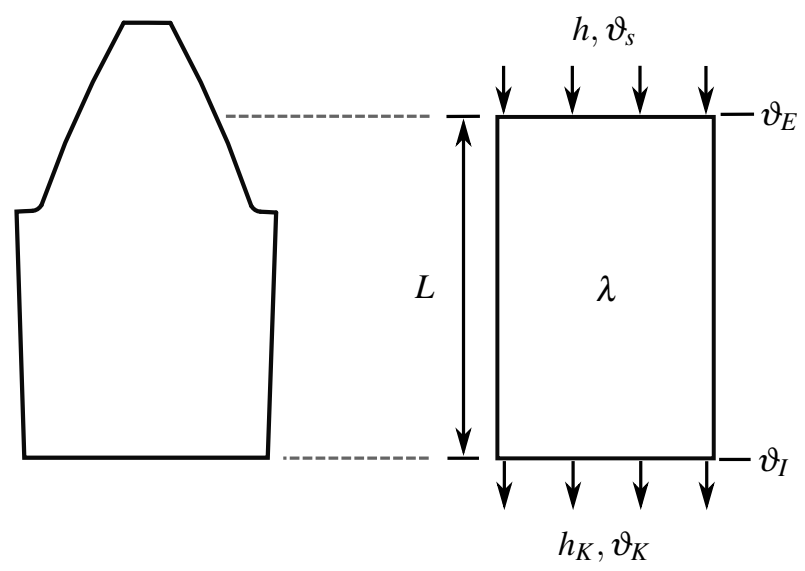

Abbildung 4.4: Eindimensionale Betrachtung des Einzelzahns 


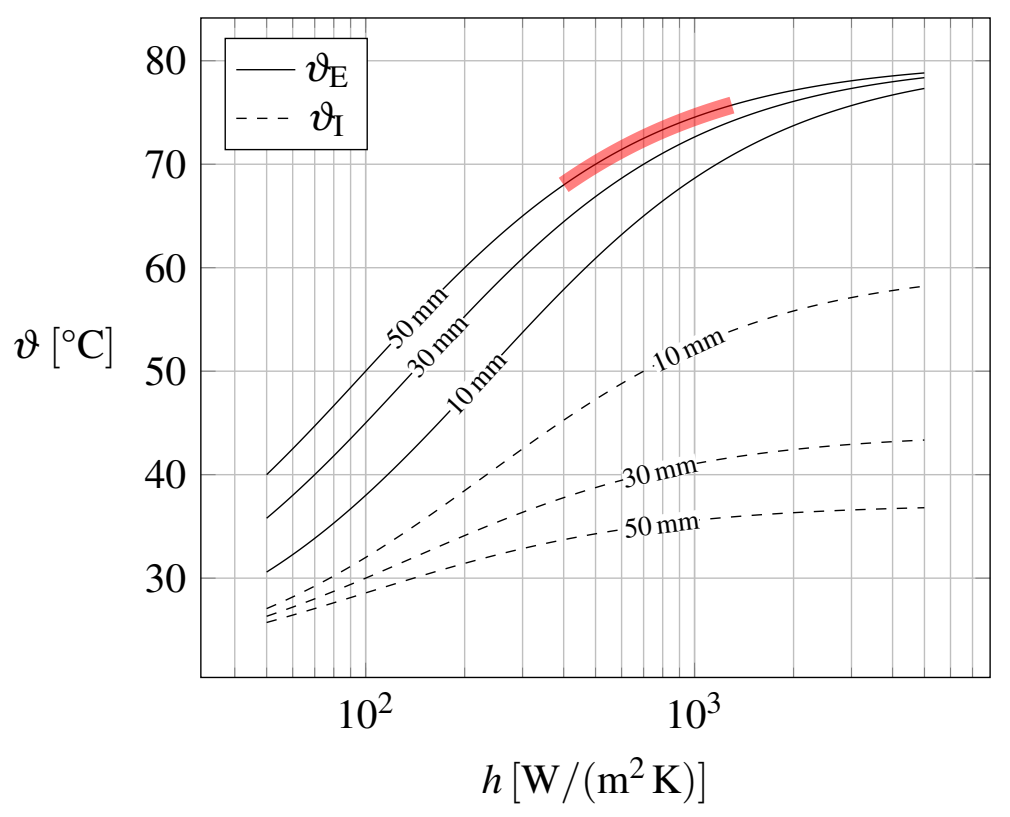

Abbildung 4.5: Verlauf der Temperaturen an den beiden Oberflächen des Ersatzkörpers, $\vartheta$ und $\vartheta_{\mathrm{K}}$, über einen Bereich von $h$ für verschiedene Prüfkörperdicken $L$ in $\mathrm{m}$

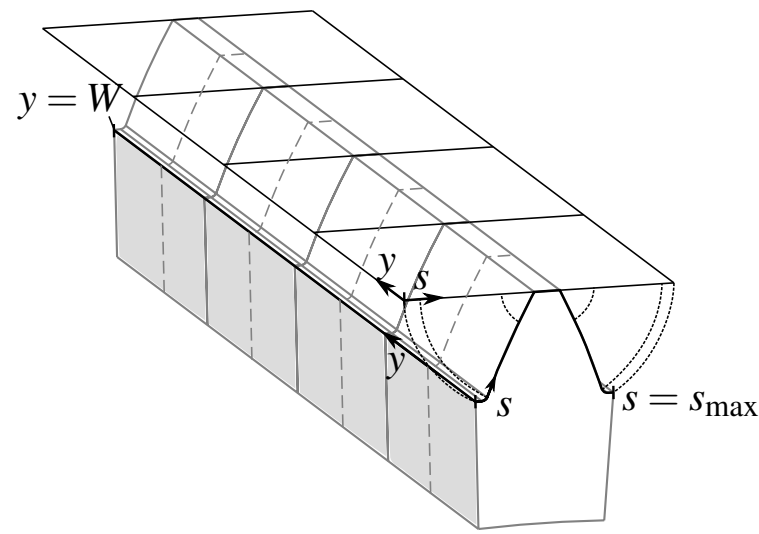

Abbildung 4.6: Flächentreues, abgewickeltes $(s, y)$ - Koordinatensystem eines Einzelzahns

In Abbildung 4.6 ist die Transformation in eine zweidimensionale Darstellung anschaulich dargestellt. Sie kommt einer Abwicklung der Zahnflanken auf eine Ebene gleich. Der Vorteil dieser Transformation gegenüber der Projektion liegt darin, dass sie flächentreu ist. Die experimentell bestimmten Wärmeübergänge sind ortsaufgelöst. Die Bestimmung des mittleren Wärmeübergangs kann im $(s, y)$-Koordinatensystem durch einfache arithmetische Mittelung erfolgen, während dies für projizierte Koordinatensysteme eine Gewichtungsfunktion erfordern würde.

Aus Gründen, die in Abschnitt 4.2 geschildert werden, sind die Temperaturmesspunkte des Versuchszahnrads über einen Viertelzahn verteilt. Dabei ist eine Hälfte dieses Viertelzahns mit 18 Messpunkten versehen, die in drei Reihen entlang der Koordinate $s^{+}$verteilt sind. Entlang der Mitte der anderen Hälfte ist eine Referenzreihe mit Messpunkten versehen. Die Verteilung der Messpunkte ist in Abbildung 4.7 dargestellt. 

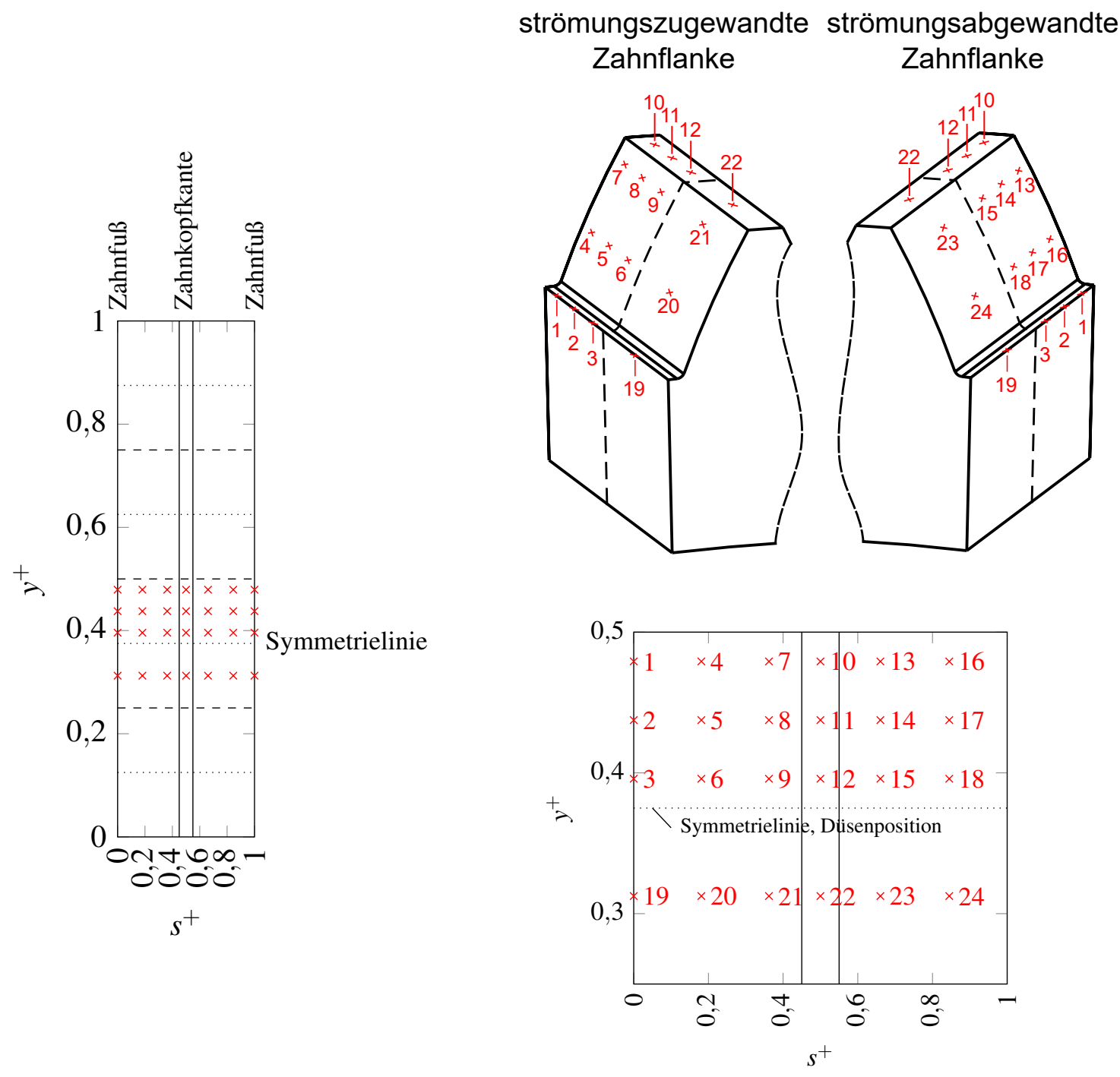

Abbildung 4.7: Verteilung der Messpunkte über einen Viertelzahn im $\left(s^{+}, y^{+}\right)$Koordinatensystem

Aus Abbildung 4.7 ist ersichtlich, dass der Bereich zwischen $y^{+}=0,375$ und $y^{+}=0,5$ mit drei Reihen mit jeweils sechs Messpunkten instrumentiert ist. Dieses Zahnachtel wird durch die iterative Berechnung ausgewertet. Bei $y^{+}=0,3125$ liegt eine weitere Reihe Messpunkte, die die Referenzmessreihe darstellt. Die dicht instrumentierte Hälfte des Viertels ist näher an der Zahnradmitte, um den Einfluss der Stirnseiten möglichst gering zu halten. Die Referenzmessreihe befindet sich mittig auf dem weiter außerhalb der Mitte befindlichen Achtel. Aufgrund der Beschränkung auf ein Zahnviertel wird in allen folgenden Abschnitten ausschließlich das Intervall $y^{+}=[0,25 ; 0,5]$ betrachtet.

Die Messpunkte des Versuchszahnrads werden durch Mantelthermoelemente vom Typ K mit einem nominellen Manteldurchmesser von 0,25 mm realisiert. Zur Applizierung der Thermoelemente ist das Versuchszahnrad mit Nuten versehen, die axial entlang der Zahnflanken vom Ort des jeweiligen Messpunkts bis zur Stirnseite des Zahnrads verlaufen. Die Thermoelemente werden mit einem Klebstoff angepasster Wärmeleitfähigkeit in die Nuten eingeklebt. Die 
Wärmeleifähigkeit des Klebstoffs ist mit 5,7 W/(mK) nur geringfügig niedriger als die des Zahnradmaterials. Dadurch und durch die sehr geringe Größe der Nuten und der Thermoelemente kann die Beeinflussung der Temperaturverteilung gering gehalten werden. Zum Einkleben eines Thermoelements wird die Nut zunächst vollständig mit Klebstoff gefüllt. Anschließend wird das Thermoelement eingeklebt. Durch Auffüllen der Nut, Aushärten des Klebstoffes und anschließendes, manuelles Abschleifen stellen sich nur sehr geringe Geometriebeeinflussungen ein.

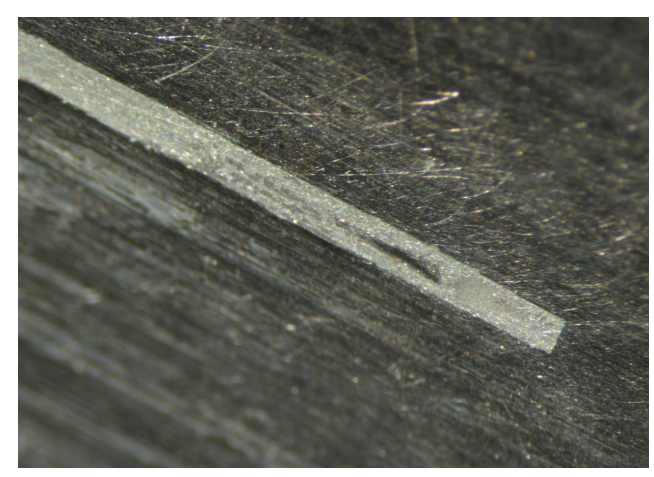

Abbildung 4.8: In Nut eingeklebtes Mantelthermoelement

Aufgrund der Anzahl an Messpunkten und konstruktiven Einschränkungen durch die Lage der Anschlusspunkte der Telemetrie können die Messpunkte nicht auf einem einzelnen Zahn angeordnet werden. Daher sind die Messpunkte über 24 Zähne verteilt. Das Temperaturfeld eines Zahns wird so nur durch eine Nut mit Thermoelement beeinflusst. Die Verteilung der Messpunkte ist in Tabelle 4.1 dargestellt. 
Tabelle 4.1: Positionen der Messpunkte und Verteilung über das Zahnrad

\begin{tabular}{llll}
\hline Messpunkt & $s^{+}$ & $y^{+}$ & Zahn \\
\hline 1 & 0,0000 & 0,4792 & 60 \\
2 & 0,0000 & 0,4375 & 61 \\
3 & 0,0000 & 0,3958 & 62 \\
5 & 0,1811 & 0,4792 & 65 \\
6 & 0,1811 & 0,4375 & 1 \\
7 & 0,1811 & 0,3958 & 2 \\
9 & 0,3621 & 0,4792 & 4 \\
10 & 0,3621 & 0,4375 & 5 \\
11 & 0,3621 & 0,3958 & 6 \\
13 & 0,5000 & 0,4792 & 29 \\
14 & 0,5000 & 0,4375 & 30 \\
15 & 0,5000 & 0,3958 & 31 \\
17 & 0,6605 & 0,4792 & 33 \\
18 & 0,6605 & 0,4375 & 34 \\
19 & 0,6605 & 0,3958 & 35 \\
21 & 0,8449 & 0,4792 & 37 \\
22 & 0,8449 & 0,4375 & 38 \\
23 & 0,8449 & 0,3958 & 39 \\
4 & 0,0000 & 0,3125 & 63 \\
8 & 0,1811 & 0,3125 & 3 \\
12 & 0,3621 & 0,3125 & 28 \\
16 & 0,5000 & 0,3125 & 32 \\
20 & 0,6605 & 0,3125 & 36 \\
24 & 0,8449 & 0,3125 & 40 \\
\hline
\end{tabular}

\subsection{Analyse der Messgenauigkeit}

Die experimentelle Umsetzung der im Vorangegangenen beschriebenen Methode unterliegt mehreren Störeinflüssen, die die Messgenauigkeit beeinflussen. Dazu gehören unter anderem Unsicherheiten in der Bestimmung der Wärmeleitfähigkeit des Materials, Messfehler in der Temperaturerfassung durch Thermoelemente und geometrische Abweichungen der Zahnradform und der Position der Messpunkte. In Arbeiten wie der von Jacobsen (1987) oder Stripf (2007) wird die aus den verschiedenen Störquellen resultierende Unsicherheit der Wärmeübergangsmessung in zwei Schritten quantifiziert. Dazu werden zunächst die Unsicherheiten der beteiligten Größen, wie etwa Temperatur oder Wärmeleitfähigkeit, durch Herstellerangaben oder Kalibriermessungen festgestellt. Anschließend wird der Einfluss dieser Einzelfehler auf den gemessenen Wärmeübergang bestimmt, indem die Wärmeleitungsgleichung entsprechend ausgewertet wird.

Eine derartige analytische Betrachtung der Fehlerfortpflanzung wird im vorliegenden Fall dadurch verhindert, dass die Geometrie des Versuchskörpers komplex ist und die Messpunkte dicht beieinander liegen. Dadurch beeinflussen Messfehler an einem bestimmten Messpunkt auch die 
ermittelten Wärmeübergangskoeffizienten an benachbarten Stellen. Dies ist ein Nebeneffekt der Auslegung des Versuchskörpers unter Annäherung von $\mathrm{Bi}=1$. Eine grobe Näherung der Unsicherheit in der Wärmeübergangsbestimmung könnte erreicht werden, indem das Versuchszahnrad modellhaft zu einem eindimensionalen Vergleichskörper vereinfacht wird. Dadurch würde jedoch die zu erwartende Messunsicherheit stark überschätzt. Die Wechselwirkung zwischen Messpunkten im vorliegenden Fall führt zu einem stochastischen Ausgleich der aufgeprägten Messunsicherheiten, wodurch die mittlere Messunsicherheit geringer ausfällt als für eine einzelne Temperaturmessung.

Die Messunsicherheit der beschriebenen Methode kann dennoch bestimmt werden, wenn sie als statistisches Problem betrachtet und mit der Monte-Carlo-Methode quantifiziert wird. Die Monte-Carlo-Methode soll im Folgenden beschrieben und die so gewonnenen Erkenntnisse über die Messgenauigkeit der vorliegenden Wärmeübergangsmessungen anschließend analysiert werden.

\subsubsection{Monte-Carlo-Methode}

Die analytische Betrachtung der Fehlerfortpflanzung setzt zwingend die geschlossene Beschreibbarkeit des jeweiligen Vorgangs voraus. In Fällen, in denen die Natur eines Vorgangs entweder unbekannt aber beobachtbar ist (z.B. Finanzmärkte) oder numerisch berechenbar, aber nicht geschlossen lösbar ist (wie im vorliegenden Fall), ist diese Voraussetzung nicht gegeben. Die Abhängigkeit solcher Vorgänge von ihren Eingangsparametern kann statistisch bestimmt werden. Bei bekannten Unsicherheiten der Eingangsgrößen ist dadurch die Unsicherheit der Ausgangsgröße bekannt - im Fall der Wärmeübergangsmessung also die Unsicherheit des Wärmeübergangskoeffizienten.

In Abbildung 4.9 ist die Vorgehensweise der Monte-Carlo-Methode zur Analyse der Fehlerfortpflanzung schematisch dargestellt. Der zu Grunde liegende Gedanke ist, dass stochastische Störungen der Eingangsgrößen eines Vorgangs sich auf eine Art in den Ausgangsgrößen niederschlagen, die nur von diesem Vorgang selbst abhängen. Um die statistische Verteilung der Ausgangsgröße zu bestimmen, wird eine große Anzahl sogenannter Ziehungen getätigt. Eine Ziehung ist eine Diskretisierung aller als Wahrscheinlichkeitsgrößen beschriebenen Eingangsgrößen. Jede Ziehung wird durch das Modell auf die Ausgangsgröße abgebildet. Die so entstandene Ausgangsgrößenverteilung kann statistisch analysiert werden, um die Messunsicherheit der Methode zu bestimmen.

Dazu werden die Eingangsgrößen des Vorgangs als stochastische Größen erfasst. Im vorliegenden Fall bedeutet dies die Quantifizierung der Messunsicherheit aller Temperaturmessungen sowie der Unsicherheit der Wärmeleitfähigkeit des Zahnradmaterials. Die numerische Berechnung der Ausgangsgrößenverteilung des Messvorgangs wurde in Abschnitt 4.3 beschrieben. Sie kann im Rahmen der Monte-Carlo-Methode als Zuordnungsvorschrift einer Einzelziehung von Eingangsgrößen zu einer Auswertung verwendet werden.

Aufgrund der so gewonnenen Daten kann die Unsicherheit des mittleren Wärmeübergangs an der Zahnoberfläche bestimmt werden. Dadurch, dass der Wärmeübergang nicht nur als Mittelwert, 


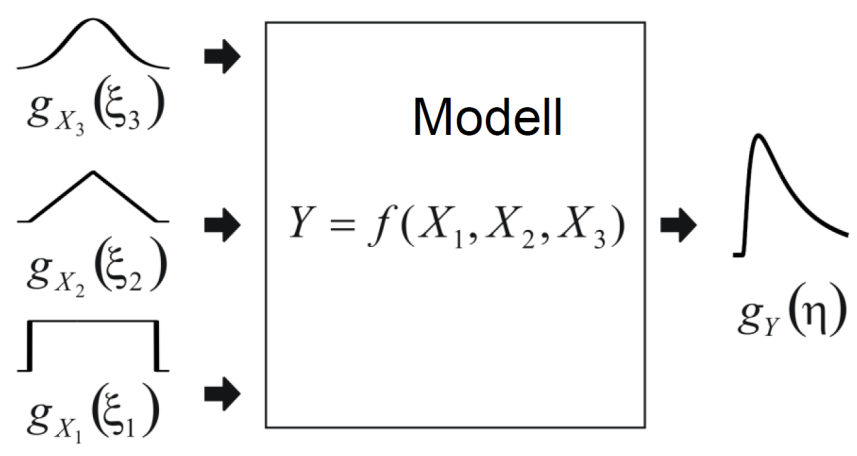

Abbildung 4.9: Fehlerfortpflanzungsanalyse durch die Monte-Carlo-Methode nach Wübbeler et al. (2008)

sondern örtlich aufgelöst bestimmt wird, kann die Messunsicherheit an jeder einzelnen Messstelle bestimmt werden. Durch die Verfügbarkeit einer hohen Anzahl an Auswertungen zufälliger Messwertvariationen können zusätzlich die Abhängigkeiten der Ausgangsgrößen von den Eingangsgrößen durch ihre jeweiligen Korrelationskoeffizienten ausgedrückt werden. Dadurch kann die Robustheit der Methode geprüft werden, indem die beobachteten Abhängigkeiten analysiert werden. Im vorliegenden Fall sind dies die Abhängigkeiten des Wärmeübergangskoeffizienten an einem Messpunkt von den gemessenen Temperaturen an diesem und den ihn umgebenden Messpunkten.

Zur Durchführung der Monte-Carlo-Simulation muss eine Wärmeübergangsverteilung angenommen werden, um eine Temperaturverteilung zu berechnen, aus der lokale Messtemperaturen extrahiert werden können. Diese berechneten Temperaturen werden anschließend für jede Ziehung mit zufälligen Messfehlern überlagert und ausgewertet. Die angenommene Wärmeübergangsverteilung ist in Abbildung 4.10a dargestellt. Die Verteilung des Wärmeübergangskoeffizienten auf der strömungszugewandten Zahnflanke entspricht entlang der $y$-Koordinate einer Sinuskurve, deren Maximum von $1000 \mathrm{~W} /\left(\mathrm{m}^{2} \mathrm{~K}\right)$ in der Mitte des Viertelzahns liegt und zu den Rändern hin auf einen Wert von Null abfällt. Auf dem Zahnkopf bei $s^{+}=0,5$ beträgt der Wärmeübergangskoeffizient $200 \mathrm{~W} /\left(\mathrm{m}^{2} \mathrm{~K}\right)$, auf der strömungsabgewandten Zahnflanke $100 \mathrm{~W} /\left(\mathrm{m}^{2} \mathrm{~K}\right)$. Die daraus resultierende, ungestörte Temperaturverteilung ist in Abbildung 4.10b dargestellt.

Die ungestörten Temperaturen, die sich aus der angenommenen Wärmeübergangsverteilung ergeben, werden für jede Ziehung mit zufälligen Messfehlern überlagert. Dabei sind für die Temperaturmessung des Öls und die Temperaturmessungen auf dem Zahnrad unterschiedliche Messunsicherheiten zu erwarten.

Die Messunsicherheit der Öltemperatur, $\delta \vartheta_{\mathrm{s}}$, setzt sich zusammen aus der Messunsicherheit der Kaltstellentemperatur, $\delta \vartheta_{\mathrm{K}}$, und der Messunsicherheit $\delta \vartheta_{\mathrm{s}, \mathrm{r}}$, die durch herstellungsbedingte Fehler des Thermoelements und die Signalwandlung entstehen. Die Häufigkeitsverteilung der zu erwartenden Messunsicherheit wird aufgrund von Herstellerangaben als Normalverteilung mit einer Standardabweichung von $\sigma=0,64 \mathrm{~K}$ abgeschätzt.

Die Signalwandlungskette der Telemetrie, mit Hilfe derer die Temperaturen am Zahnrad gemessen werden, ist wesentlich komplexer als die des Signalwandlers der Öltemperatur. Durch 


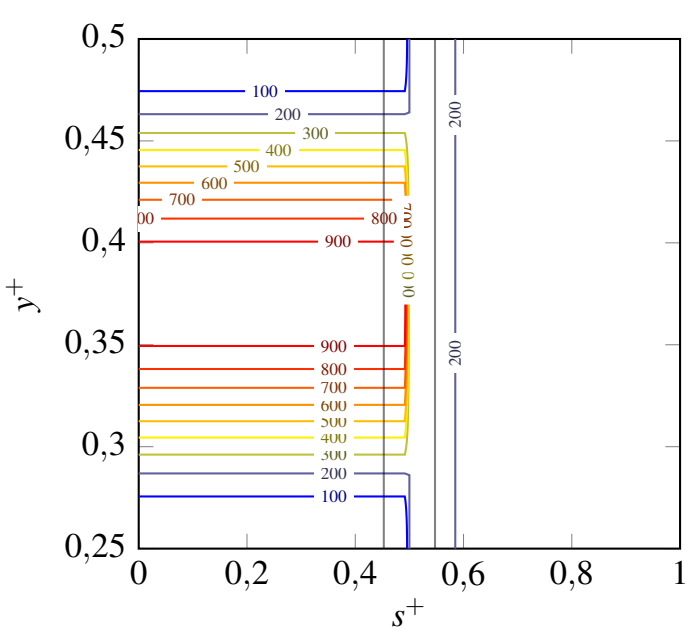

(a) Angenommener Wärmeübergang in $\mathrm{W} /\left(\mathrm{m}^{2} \mathrm{~K}\right)$

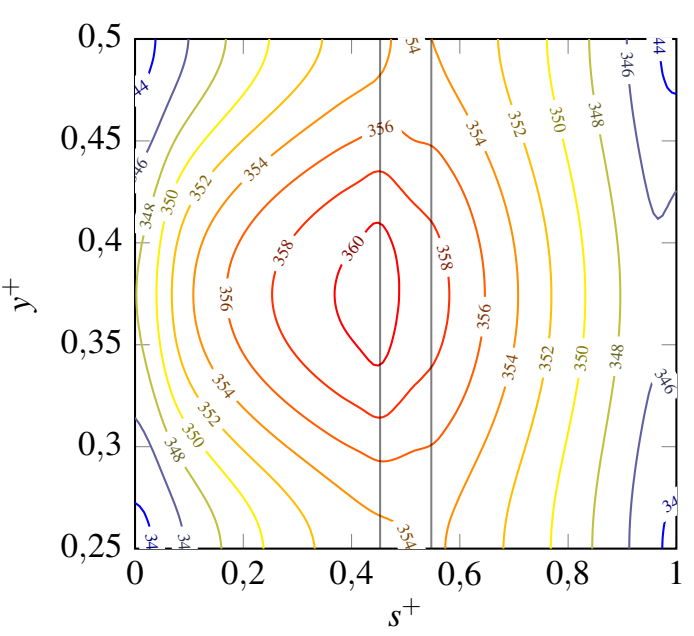

(b) Berechnete Temperaturverteilung in $\mathrm{K}$

Abbildung 4.10: Angenommener Wärmeübergang und berechnete Temperaturverteilung für die Monte-Carlo-Simulation

Kalibrierdaten des Herstellers kann jedoch die Unsicherheit $\delta U_{\mathrm{TE}}$ bestimmt werden. Die Unsicherheit der Messung der Temperaturen an der Zahnoberfläche wird durch eine Wahrscheinlichkeitsdichtefunktion, die aus Kalibrierdaten gewonnen wird, abgebildet. Die Standardabweichung der Messung beträgt $\sigma=0,21 \mathrm{~K}$.

Aufgrund des Rechenaufwands, der pro Ziehung bei etwa 15 Minuten liegt, und der Vielzahl an notwendigen Ziehungen wird im vorliegenden Fall das Stein'sche Schema zur Reduktion des Rechenaufwands verwendet (Wübbeler et al. (2010)). Bei Anwendung dieses Verfahrens werden zunächst einige Blöcke, bestehend aus etwa $10^{1}$ bis $10^{2}$ Ziehungen, berechnet. Aufgrund der statistischen Verteilung der Blockmittelwerte kann anschließend eine Aussage über die benötigte Gesamtzahl an Ziehungen getroffen werden, die zum Erreichen eines definierten Konfidenzintervals der Schätzung der Messunsicherheit ausgewertet werden müssen (Wübbeler et al. (2010)). Im vorliegenden Fall ergibt die Auswertung von 8 Blöcken mit jeweils 50 Ziehungen eine benötigte Gesamtzahl von 5650 Ziehungen, um die Unsicherheit der Methode zu charakterisieren. Dabei wird eine Charakterisierung der Unsicherheit mit einer Abweichung von $1 \%$ und einem Konfidenzintervall von $95 \%$ angestrebt.

\subsubsection{Unsicherheit der Wärmeübergangsmessungen}

Auf Grundlage der zuvor genannten Unsicherheiten der Eingangsgrößen kann durch die MonteCarlo-Methode die Unsicherheit der Wärmeübergangsmessungen bestimmt werden. Dabei können sowohl die Unsicherheit des mittleren Wärmeübergangs als auch die lokalen Messunsicherheiten an den Messpunkten betrachtet werden.

Zunächst soll die Unsicherheit des gemittelten Wärmeübergangs betrachtet werden. In der Arbeit von Niehues (2018) wird der verwendete zweistufige Stein'sche Ansatz zur Bestimmung der Messunsicherheitsverteilung beschrieben, dessen Ergebnisse im Folgenden ausgewertet werden 
sollen.

Die in das Zahnrad übertragenen lokalen Wärmeströme, die mit der iterativen Methode berechnet werden, bilden zusammen mit der Öltemperatur und der lokalen Oberflächentemperatur den Wärmeübergangskoeffizienten $h$. Die Unsicherheit der Messung der Öltemperatur hat dadurch einen Einfluss auf die Unsicherheit des mittleren Wärmeübergangs. Die Häufigkeitsverteilung des mittleren Wärmeübergangskoeffizienten $\bar{h}$ aus allen Ziehungen der Monte-Carlo-Simulation ist in Abbildung 4.11a dargestellt. Die Verteilung entspricht einer Normalverteilung mit dem Mittelwert $\bar{h}_{\mathrm{m}}=298,80 \mathrm{~W} /\left(\mathrm{m}^{2} \mathrm{~K}\right)$ und einer Standardabweichung von $\sigma=11,24 \mathrm{~W} /\left(\mathrm{m}^{2} \mathrm{~K}\right)$ oder $\sigma=3,76 \%$ des Mittelwerts. Aus Abbildung $4.11 \mathrm{~b}$ ist ersichtlich, dass die Öltemperatur $\vartheta_{\mathrm{s}}$ stark mit dem mittleren Wärmeübergangskoeffizienten $\bar{h}$ korreliert. Der Korrelationskoeffizient beträgt $r=-0,9815$.

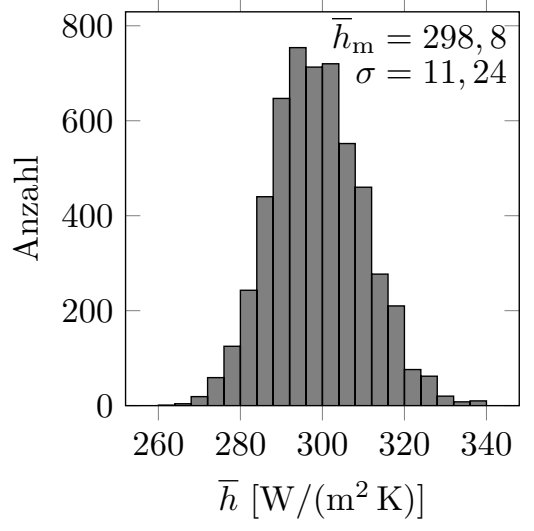

(a) Häufigkeitsverteilung

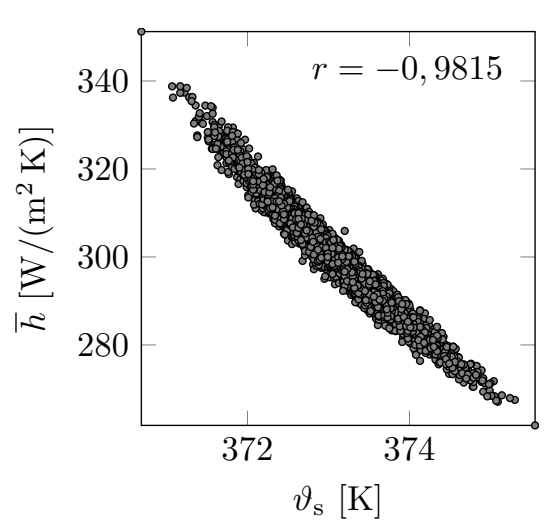

(b) Verteilung von $\bar{h}$ in Abhängigkeit der Öltemperatur $\vartheta_{\mathrm{s}}$

Abbildung 4.11: Messunsicherheit des mittleren Wärmeübergangs

Aufgrund der Geometrie des Zahnrads und der Auslegung zu Bi $\approx 1$ sind die Unsicherheiten der lokalen Wärmeübergangsmessung von den Messunsicherheiten aller Eingangsparameter abhängig. Jedoch kann gezeigt werden, dass die Öltemperatur und die lokale Temperatur einen dominierenden Einfluss auf die Messunsicherheit haben und die benachbarten Messpunkte sie nur nachrangig beeinflussen.

Die Korrelationskoeffizienten der berechneten lokalen Wärmeübergangskoeffizienten $h_{i}$ und der Öltemperatur sind in Abbildung 4.12 dargestellt. Die Öltemperatur korreliert dabei stets negativ mit den Wärmeübergangskoeffizienten. Messpositionen hohen Wärmeübergangs weisen eine stärkere Abhängigkeit von der Öltemperatur auf, da die Temperaturdifferenz zwischen Oberfläche und Öltemperatur an diesen Positionen niedriger ist. Die Temperaturdifferenz, die in den Nenner des Wärmeübergangskoeffizienten eingeht, ist somit kleiner (vgl. Gleichung 4.6). 

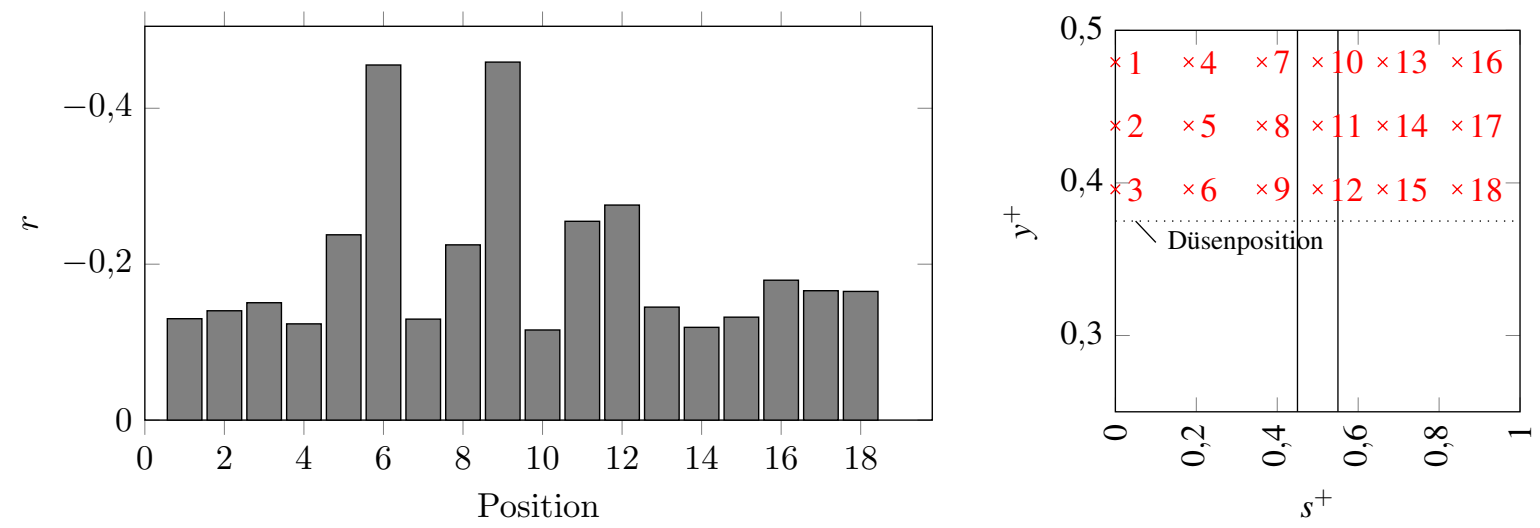

Abbildung 4.12: Abhängigkeit des lokalen Wärmeübergangskoeffizienten von der Messunsicherheit der Öltemperatur

Die Streuung der Wärmeübergangskoeffizienten an Messpunkt 9 ist in Abbildung 4.13a dargestellt. Der betrachtete Messpunkt liegt in der Nähe der Zahnmitte und nah am Zahnkopf auf der strömungszugewandten Zahnflanke. Die Verteilung der gemessenen Wärmeübergangskoeffizienten entspricht einer Normalverteilung mit dem Mittelwert $h_{9, \mathrm{~m}}=1057,3 \mathrm{~W} /\left(\mathrm{m}^{2} \mathrm{~K}\right)$ und einer Standardabweichung von $\sigma=112,9 \mathrm{~W} /\left(\mathrm{m}^{2} \mathrm{~K}\right)$. Aus Abbildung $4.13 \mathrm{~b}$ wird deutlich, dass der ermittelte Wärmeübergangskoeffizient stark von der lokalen Temperatur abhängt. Mit einem Korrelationskoeffizienten von $r=0,8189$ hat die lokale, gemessene Temperatur den größten Einfluss auf die Messunsicherheit des lokalen Wärmeübergangskoeffizienten.

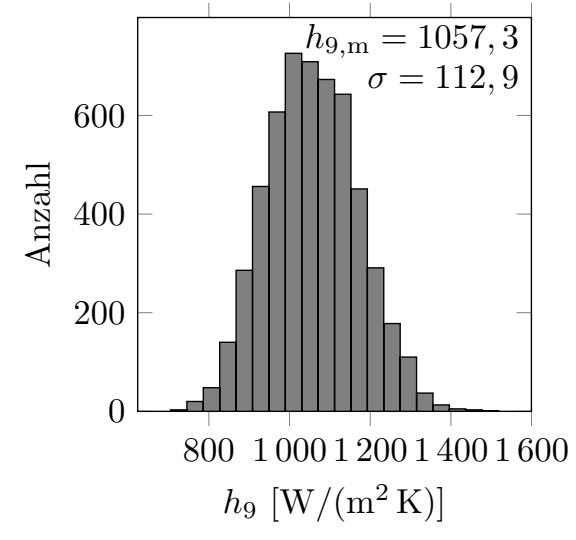

(a) Häufigkeitsverteilung

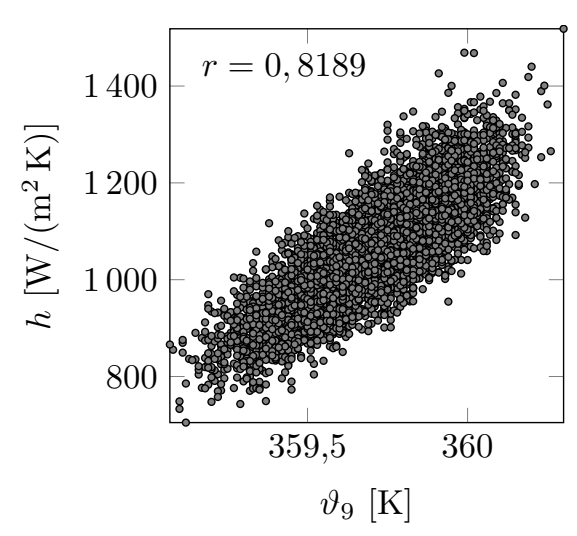

(b) Verteilung von $h$ in Abhängigkeit der lokalen Temperatur an der Messposition 9

Abbildung 4.13: Unsicherheit des lokalen Wärmeübergangs an der Messposition 9

Durch die Darstellung der Korrelationskoeffizienten des gemessenen Wärmeübergangskoeffizienten an Position 9 und aller gemessenen Temperaturen in Abbildung 4.14 wird deutlich, dass auch die Temperaturen der nächstgelegenen Temperaturmesspunkte 6,8 und 12 den an Position 9 gemessenen Wärmeübergangskoeffizienten beeinflussen. Dies ist eine Auswirkung des beabsichtigten örtlichen „Mittelungseffektes“ bei $\mathrm{Bi} \approx 1$. Die weiter entfernt gelegenen Messpunkte beeinflussen den lokalen Wärmeübergangskoeffizienten kaum. 

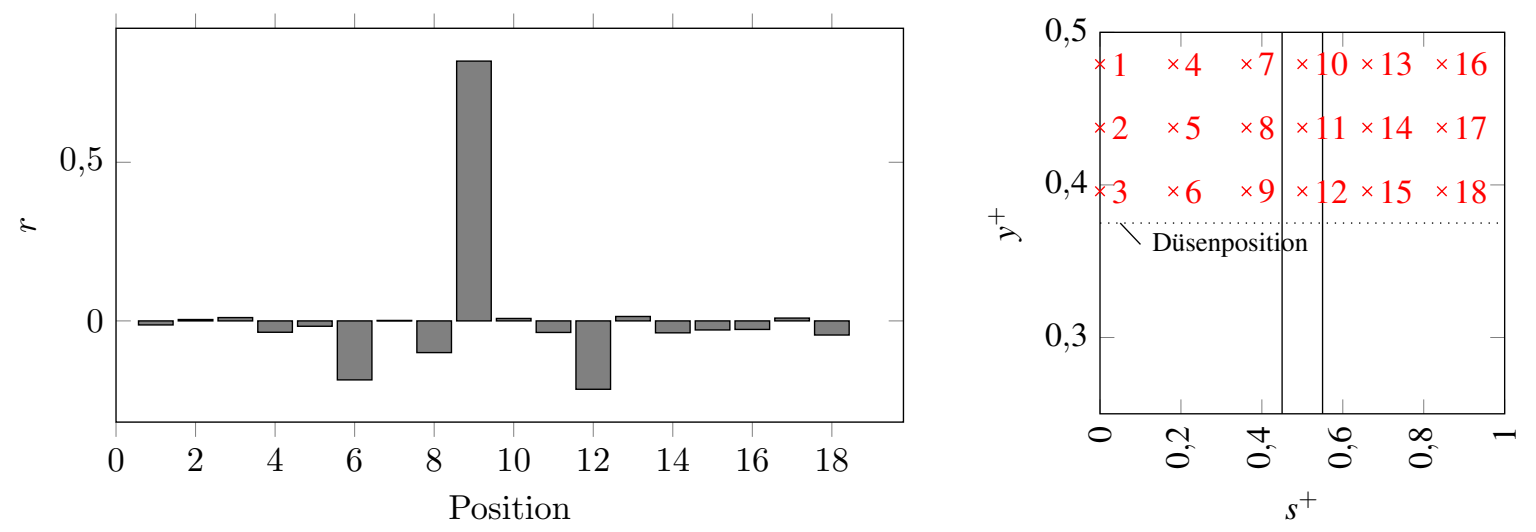

Abbildung 4.14: Korrelationskoeffizienten des Wärmeübergangs an Position 9 und der Temperaturen an den Messpunkten

Die beobachteten Einflüsse auf die Unsicherheit der lokalen Wärmeübergangsmessung an Position 9 werden auch an allen anderen Messpunkten beobachtet. Die näher am Zahnfuß gelegenen Messpunkte zeigen dabei tendenziell weiträumigere Abhängigkeit von anderen Messstellen. Die ausführliche Diskussion der jeweiligen Unsicherheiten und Korrelationskoeffizienten soll an dieser Stelle jedoch nicht erfolgen. In Abbildung 4.15 ist $|r|$ für alle Kombinationen von $\vartheta_{i}$ und $h_{i}$ dargestellt. Werte der Hauptdiagonale stellen darin die Abhängigkeit eines berechneten, lokalen Wärmeübergangskoeffizienten $h_{i}$ von der gemessenen Temperatur $\vartheta_{i}$ an der Position $i$ dar. Durch diese Form der Darstellung wird deutlich, dass die lokale Temperatur stets den größten Einfluss auf den berechneten Wärmeübergangskoeffizienten hat.

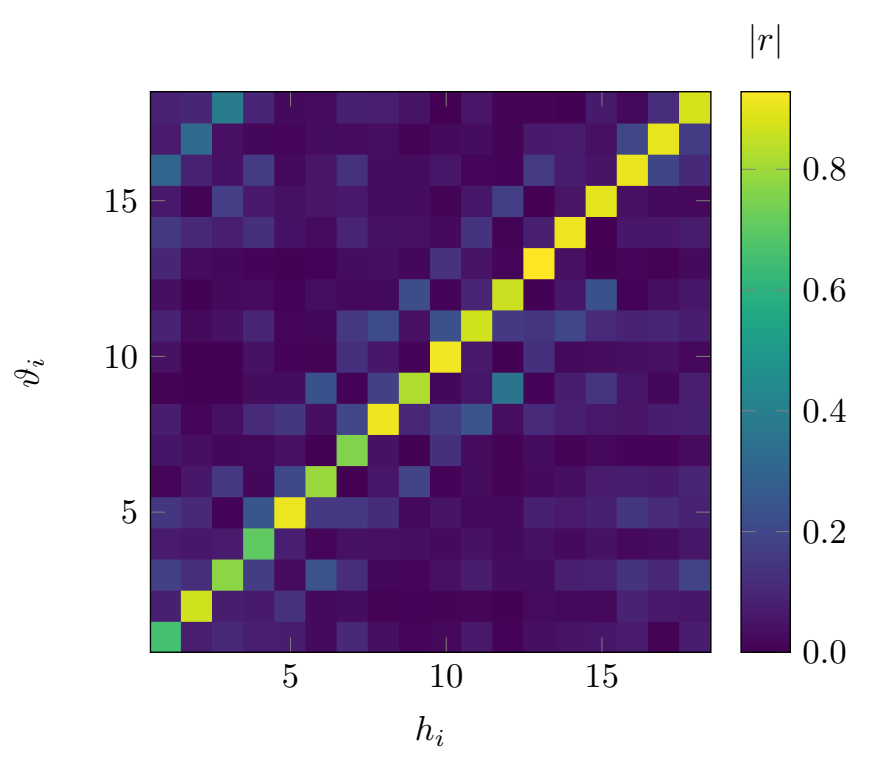

Abbildung 4.15: Korrelationskoeffizienten aller lokalen Wärmeübergangskoeffizienten von allen Oberflächentemperauren 


\subsubsection{Zusammenfassung}

Die vorangegangene Betrachtung liefert die Erkenntnis, dass die Messunsicherheit des mittleren Wärmeübergangs vergleichsweise gering ist. Für den verwendeten Testfall wird eine Standardabweichung von $\sigma=3,76 \%$ ermittelt. Die Verteilung und der Wertebereich des Testfalls sind den gemessenen Wärmeübergängen dieser Arbeit ähnlich. Die Unsicherheit der Öltemperaturmessung hat den größten Einfluss auf die Messunsicherheit des Wärmeübergangs. Durch Kalibrierung der verwendeten Temperaturmesskette für die Öltemperatur wird eine wesentlich geringere Unsicherheit der Öltemperatur festgestellt. Es ist daher davon auszugehen, dass die tatsächliche Unsicherheit des mittleren Wärmeübergangs ebenfalls wesentlich geringer als die durch die Monte-Carlo-Simulation bestimmte Unsicherheit ist. Die Unsicherheiten der lokalen Wärmeübergangsmessungen übersteigen die des mittleren Wärmeübergangs erheblich. Sie werden am stärksten von den lokalen Temperaturmessungen und der Messung der Öltemperatur beeinflusst. Die Unsicherheiten der jeweils benachbarten Messpunkte haben ebenfalls einen eindeutigen, jedoch geringeren Einfluss. 


\section{Versuchsaufbau und Messtechnik}

Aus Kapitel 2 geht hervor, dass die Charakterisierung des Öl-Zahnrad-Wärmeübergangs für das Erreichen höchster Leistungsdichten in Getrieben unabdingbar ist. Die im Rahmen der vorliegenden Arbeit untersuchte Ausgestaltung einer Einspritzkühlung wird in Kapitel 3 beschrieben. In Kapitel 4 wird die experimentelle Methode beschrieben, die die Messung des Wärmeübergangs zwischen Öl und Zahnrad ermöglicht. Die zur Erfüllung der Zielsetzung dieser Arbeit notwendige Implementierung dieser Methode in Form eines Versuchsstands und einer Versorgungsanlage soll im Folgenden beschrieben werden.

\subsection{Implementierung in einem Versuchsstand}

Zur Umsetzung der in Kapitel 4 beschriebenen Methode sind folgende Anforderungen an Versuchsstand und Versorgungsanlagen zu erfüllen:

- Einspritzen des beheizten Öls

- Variation des Düsenwinkels

- Auffangen und Abführen des Öls

- Kühlen des Versuchszahnrads

- Antrieb des Versuchszahnrads

- Ermöglichen von Gegenlichtaufnahmen

- Messen von Temperaturen im rotierenden System

Aus der Beschreibung der Methode in Kapitel 4 wird deutlich, weshalb ein Temperaturgradient zur Messung des Wärmeübergangs an einem einzelnen Zahnrad erzeugt werden muss. Dies geschieht im vorgestellten Versuchsstand durch Heizen des Öls und Kühlen des Zahnradinnenradius, was eine Umkehr der Wärmestromrichtung im Vergleich zum Anwendungsfall darstellt. So können die Luftversorgung, in der keine Heizung benötigt wird, und die Prüfstandskonstruktion, die geringeren Temperaturen standhalten muss, einfacher gestaltet werden. Es werden Betriebsbedingungen angestrebt, die denen existierender Höchstleistungsgetriebe in Triebwerken entsprechen. Die Betriebsgrenzen des Versuchsstands sind in Tabelle 5.1 zusammengefasst.

Um die genannten Anforderungen zu erfüllen, wurde der in Abbildung 5.1 dargestellte Versuchsstand entworfen und am Institut für Thermische Strömungsmaschinen gefertigt.

Das Versuchszahnrad, das in Kapitel 4 beschrieben wird, ist durch eine Wälzlagerung gelagert und wird vom Antriebsmotor über eine Drehmomentmesswelle direkt angetrieben. Durch die „fliegende“ Lagerung der Drehmomentmesswelle führen Ausrichtungsfehler, die nicht vollständig 
Tabelle 5.1: Betriebsgrenzen des Versuchsstands

\begin{tabular}{lcr}
\hline Parameter & Symbol & Wert \\
\hline Drehzahl & $n$ & $6500 / \mathrm{min}$ \\
Ölvolumenstrom & $\dot{V}$ & $151 / \mathrm{min}$ \\
Düsenwinkel & $\alpha$ & $-45^{\circ} \mathrm{bis} 45^{\circ}$ \\
Öltemperatur & $\vartheta_{\mathrm{s}}$ & $100^{\circ} \mathrm{C}$ \\
\hline
\end{tabular}

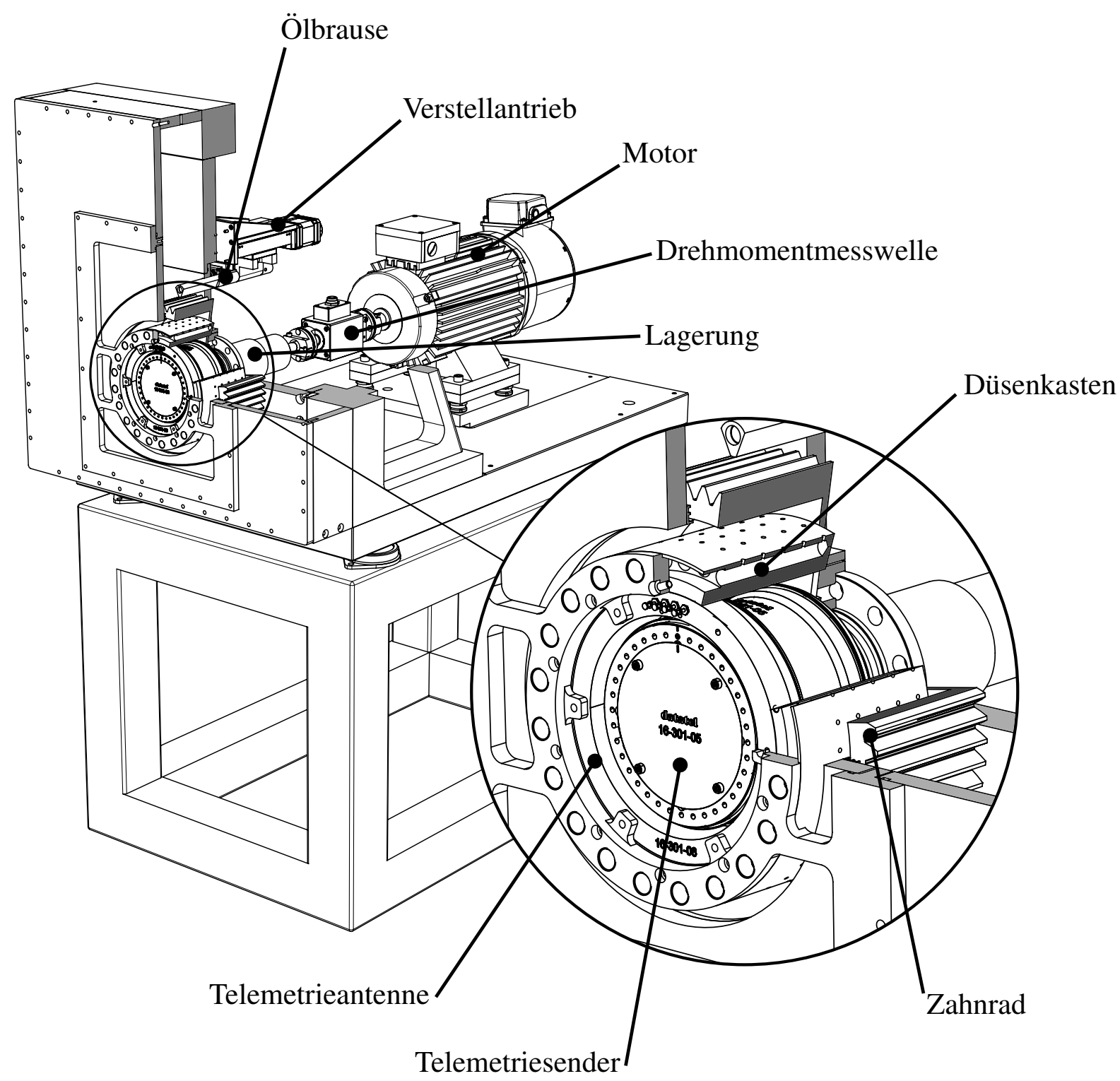

Abbildung 5.1: Schematische Darstellung des eingesetzten Versuchsstands zur Messung des Öl-Zahnrad-Wärmeübergangs

zu vermeiden sind, nicht zu umlaufenden Biegemomenten im Antriebsstrang, was zu einem äußerst schwingungsarmen Betrieb führt.

Eine stirnseitige Labyrinthdichtung dichtet die ölhaltige Umgebung, in der sich die verstellbare 
Ölbrause befindet, gegen die Umgebung des Prüfstands ab. Innerhalb des hohlen Zahnrads befindet sich ein zylinderförmiger Düsenkasten, der zur Prallkühlung der Innenfläche des Zahnrads durch einen Seitenkanalverdichter mit Luft versorgt wird. Die Vorderseite des Versuchsstands ist mit einem Fensterrahmen versehen, der als integrales Bauteil sowohl den Düsenkasten als auch die Telemetrieantenne aufnimmt. Der Telemetriesender ist in das Zahnrad integriert.

Die Ölbrause ist oberhalb des Zahnrads angebracht. Sie ist schwenkbar gelagert und kann, ausgehend von der Neutrallage, durch einen Verstellantrieb während des Versuchs um $45^{\circ}$ in beide Richtungen geschwenkt werden. Die Neutrallage ist dabei die Lage, in der der Ölstrahl in Richtung der Zahnradachse gerichtet ist. Die Ölbrause besitzt vier Auslässe, die in Abständen von jeweils $0,25 \mathrm{~W}$ gleichmäßig über die Tiefe des Zahnrads $W=90 \mathrm{~mm}$ angeordnet sind (siehe Abbildung 5.2) und einen Auslassdurchmesser von $d=1,15 \mathrm{~mm}$ bei einer Bohrungslänge von $l=7 \mathrm{~mm}$ besitzen. Die Düsenbohrungen werden durch Hartmetallbohrer gefertigt, erst anschließend wird die Auslassfläche gefräst. Dadurch kann sichergestellt werden, dass der Auslass gratfrei ist. Die Ölzuführung zu den Düsen erfolgt über eine Kernbohrung mit einem Durchmesser von $10 \mathrm{~mm}$, die stirnseitig in die Ölbrause eingebracht und mit einem hartgelöteten Deckel verschlossen wird. Durch boroskopische Inspektion wird die Freiheit von Riefen und Graten innerhalb der Düse sichergestellt. Die Ölbrause wird durch eine flexible Leitung, die die Schwenkbewegung ermöglicht, und eine rückseitige Kernbohrung mit Öl versorgt. Die vorderseitige und die rückseitige Kernbohrung weisen einen Achsabstand von 2,1 mm auf. Durch numerische Untersuchungen kann gezeigt werden, dass durch den Achsabstand und die dadurch entstehende Stufe nur sehr geringe Störungen in der Anströmung der Düsen entstehen (Kanzow (2017)). Durch ein mittig in den Einlass der Ölbrause eingelassenes Thermoelement kann die Öltemperatur unmittelbar vor den Düsen gemessen werden.

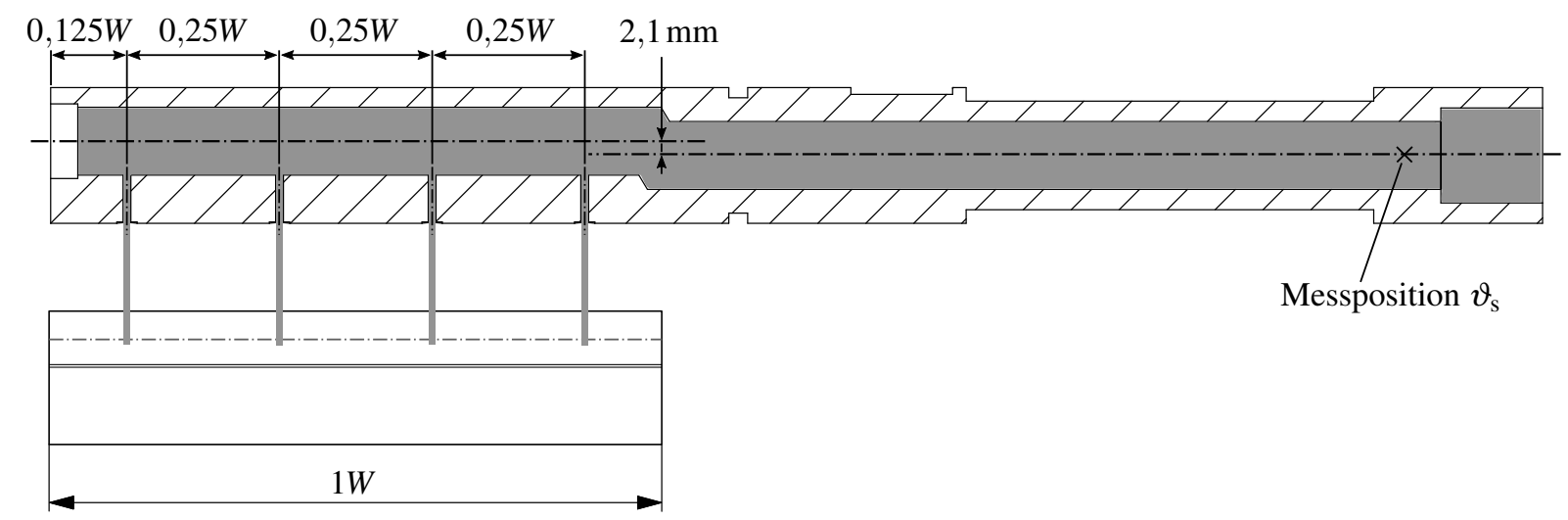

Abbildung 5.2: Schnittdarstellung der Ölbrause mit Zuführbohrung durch die Düsenebene

Durch ein vorderseitiges und ein rückseitiges Fenster und unter Verwendung einer Gegenlichtquelle können Hochgeschwindigkeits-Schattenbildaufnahmen des Einspritzvorgangs aufgenommen werden. Die Ölbrause ist so gestaltet, dass sich der Austritt aus den Öldüsen auf dem größten Durchmesser der Ölbrause befindet. Der Ölstrahl, der die Ölbrause verlässt, ist somit in den Aufnahmen auf seiner gesamten Länge sichtbar. Durch die Verwendung eines geradverzahnten Zahnrads ist das Auftreffen des Ölstrahls auf die Zahnradoberfläche beobachtbar. Die verwendete Hochgeschwindigkeitskamera vom Typ LaVision HighSpeedStar 8 ermöglicht Aufnahmese- 
quenzen mit einer Bildrate von $27 \mathrm{kHz}$ bei Bildausschnitten mit 512x512 Pixeln. Dadurch sind Aufnahmen möglich, mit denen die Ausbreitung und das Auftreffen des Ölstrahls mit mehreren Einzelbildern aufgenommen werden können. Bei $n=3000 / \mathrm{min}$ sind so beispielsweise acht Einzelbilder eines Auftreffvorgangs erfassbar.

\subsection{Anlagen zur Ölversorgung und Luftkühlung}

Die Bereitstellung des benötigten heißen Öls erfolgt über eine Versorgungsanlage, die in Abbildung 5.3 schematisch dargestellt ist. Durch den Heizkreislauf, bestehend aus einer Zahnradpumpe und einer elektrischen Heizung, wird das Öl aus dem Tank gefördert, erwärmt und in den Tank zurückgeführt. Über einen PID-Prozessregler wird die Temperatur am Heizungsauslass auf einen Sollwert geregelt. Die Versorgung des Versuchsstands erfolgt über den Versorgungskreislauf, bestehend aus einer Zahnradpumpe, einem Filter und einem hochgenauen Coriolis-Massendurchflusssensor. Die Förderleistung der Zahnradpumpe wird durch einen Frequenzumrichter und einen PID-Prozessregler geregelt, um einen voreingestellten Massendurchfluss zu erreichen. Zur Sicherstellung von Blasenfreiheit wird die Dichte des Öls, die durch den Massendurchflusssensor gemessen wird, überwacht. Die ölführenden Leitungen der Versorgungsanlage sind nahezu vollständig mit Isoliermaterial ummantelt, um Temperatur- und Dichteunterschiede sowie thermische Verluste gering zu halten. Eine weitere Zahnradpumpe saugt das Öl, das den Boden des Versuchsstands erreicht, durch drei Ablaufbohrungen ab.

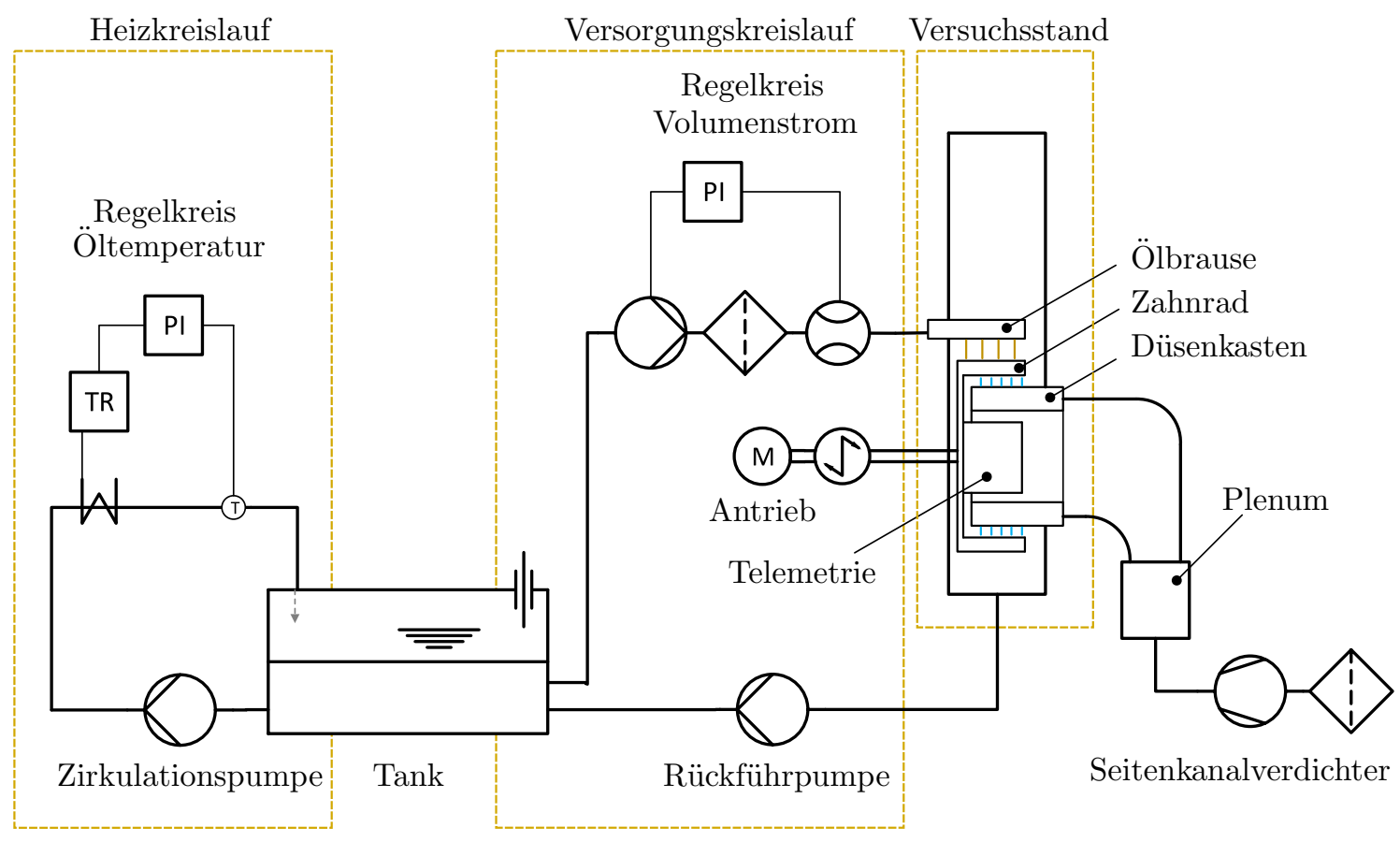

Abbildung 5.3: Anlagenplan des eingesetzten Versuchsstands zur Messung des Öl-ZahnradWärmeübergangs

Um die notwendige Kühlung des Zahnrads auf seiner Innenfläche, die in Kapitel 4 erläutert wird, zu gewährleisten, wird der Zahnradinnenmantel durch einen Düsenkasten mit gleichmäßig 
angeordneten Auslassbohrungen gekühlt. Der Düsenkasten und das Zahnrad sind konzentrisch angeordnet. Jeweils fünf Auslassbohrungen sind in einer axialen Reihe angeordnet und werden durch eine gemeinsame Bohrung mit Luft versorgt. Der Düsenkasten besitzt 40 dieser Reihen, wodurch insgesamt 200 Kühlluftbohrungen mit einem Durchmesser von jeweils 2,5 mm zur Kühlung eingesetzt werden. Der radiale Abstand der Auslassbohrungen zum Zahnrad beträgt 15 mm. Über ein Plenum im Düsenkasten und 20 Schlauchanschlussstücke ist der Düsenkasten über einen Luftsammler mit dem Seitenkanalverdichter verbunden.

\subsection{Messtechnik}

Die Messung der Temperaturen am Zahnrad und die Berechnung des Wärmeübergangs sowie die dabei herrschende Messunsicherheit werden in Kapitel 4 beschrieben. Die restlichen Betriebsparameter unterliegen ebenfalls messtechnischen Unsicherheiten, die im Folgenden beleuchtet werden sollen. Die Genauigkeiten der jeweiligen Messinstrumente sind in Tabelle 5.2 aufgeführt.

\section{Ölvolumenstrom}

Die Messung des Ölvolumenstroms erfolgt durch einen Durchflussmesser vom Typ Krohne Optimass 6400C. Der Sensor arbeitet nach dem Coriolis-Prinzip, durch das bei einphasigen Fluiden der Massenstrom und die Dichte direkt gemessen werden. Der Volumenstrom wird aus den beiden Größen berechnet. Dadurch, dass die Dichte des Fluids stets gemessen wird, kann durch einen Abgleich mit den Stoffdaten des verwendeten Öls sichergestellt werden, dass keine Luftblasen im Öl vorhanden sind. Diese können aufgrund des Aufbaus der Versorgungsanlage als Kreislauf und durch die Mischung von Öl und Luft prinzipiell auftreten. In den Experimenten, die in der vorliegenden Arbeit beschrieben werden, kann jedoch zu keinem Zeitpunkt eine Dichteänderung des Öls aufgrund von Luftblasen festgestellt werden.

\section{Öltemperatur}

Die Temperatur des Öls wird sowohl im Heizkreislauf als auch in der Zuführbohrung der Öldüse gemessen. Dabei kommen Mantelthermoelemente vom Typ K mit einem Manteldurchmesser von $0,5 \mathrm{~mm}$ zum Einsatz. Diese sind über Ausgleichsleitungen mit dem Prozessregler bzw. einem Signalerfassungsmodul vom Typ National Instruments NI-9211 verbunden. Die Kaltstellenkompensation erfolgt über integrierte temperaturabhängige Widerstände.

\section{Drehmoment und Drehzahl}

Die Drehzahl des Zahnrads und das Antriebsdrehmoment werden durch eine Drehmomentmesswelle vom Typ Lorenz DR-3000 gemessen. Zur Schwingungsreduktion ist der Sensor zwischen zwei Lamellenkupplungen angebracht und nicht fest mit der Bodenplatte des Versuchsstands verbunden.

\section{Düsenwinkel}

Die Ölbrause ist schwenkbar gelagert und wird durch einen Servoantrieb verstellt. Über ein Potentiometer, das koaxial zur Antriebswelle des Servos und in den Servo integriert angebracht ist, kann der Drehwinkel der Düse ausgelesen werden. Das Spannungssignal des Drehwinkels wird über ein Signalerfassungsmodul vom Typ NI-9201 erfasst. 
Tabelle 5.2: Genauigkeit

\begin{tabular}{lcrr}
\hline Parameter & Gerät & Bereich & Genauigkeit \\
\hline Ölvolumenstrom & Krohne Optimass 6400C & $0-201 / \mathrm{min}$ & $\pm 0,05 \%$ \\
Drehzahl & Lorenz DR-3000 & $0-20000 / \mathrm{min}$ & $\pm 1 \%$ \\
Öltemperatur (Düse) & Thermoelement Typ K & $-270-1300^{\circ} \mathrm{C}$ & $\sigma=0,5^{\circ} \mathrm{C}$ \\
Düsenwinkel & Servoantrieb / Potentiometer & $-45-45^{\circ}$ & $\pm 2 \%$ \\
\hline
\end{tabular}

\section{Hochgeschwindigkeitsaufnahmen}

Um die Interaktion des Ölstrahls mit dem Zahnrad zu charakterisieren und das Verhalten des Wärmeübergangs einordnen zu können, ist der Versuchsstand mit vorder- und rückseitigen Fenstern ausgestattet. Das Zahnrad und die Ölbrause sowie der Antrieb der Ölbrause sind so gestaltet, dass keine optischen Versperrungen zwischen der Austrittsfläche des Öls und der Zahnkontur vorhanden sind. Dadurch sind Hochgeschwindigkeitsaufnahmen im Gegenlichtverfahren möglich. Zur Aufzeichnung dieser Gegenlichtaufnahmen werden eine LED-Hintergrundbeleuchtung vom Typ Phlox HSC und eine Hochgeschwindigkeitskamera vom Typ LaVision HighSpeedStar 8 eingesetzt.

\section{Temperaturen im rotierenden System}

Zur Erfassung der Zahnradtemperaturen auf den Zahnflanken und dem Innenradius des Zahnrads ist die kabellose Übertragung der Signale notwendig. Das verwendete Telemetriesystem ermöglicht die Erfassung von 28 Temperaturkanälen. Dabei wird die Kaltstellentemperatur über einen der Kanäle erfasst. Die Erfassung der Temperaturen erfolgt dabei sequentiell mit einer Rate von 1,6 Hz. Der Aufbau der Senderelektronik bewirkt eine Mittelung des Thermospannungssignals. Zur Reduktion des Rauschens wird das digitalisierte Signal empfängerseitig über einen Ringspeicher gemittelt. 


\section{Ergebnisse}

Um die Zielsetzung dieser Arbeit zu erfüllen, wird im Folgenden der Öl-Zahnrad-Wärmeübergang experimentell untersucht. Durch Variation der Betriebsparameter kann in dieser Arbeit erstmalig das charakteristische Verhalten des Öl-Zahnrad-Wärmeübergangs in Abhängigkeit der Betriebsparameter beschrieben werden.

Der Wärmeübergang an der Zahnradoberfläche wird nach der in Kapitel 4 beschriebenen Methode ermittelt. Zusätzlich werden Hochgeschwindigkeitsaufnahmen unter Verwendung einer Gegenlichtquelle aufgezeichnet, um Rückschlüsse auf die auftretende Strömung und die ÖlZahnrad-Interaktion zu ziehen.

Für die Bestimmung des Wärmeübergangs werden die Betriebsbedingungen durch die in Kapitel 5 beschriebenen Anlagen erzeugt. Variable Betriebsbedingungen im Rahmen dieser Untersuchung sind:

- Drehzahl $n$

- Ölvolumenstrom $\dot{V}$

- Düsenwinkel $\alpha$

- Öltemperatur $\vartheta_{\mathrm{s}}$.

Die untersuchten Betriebspunkte sind in Abbildung 6.1 dargestellt.

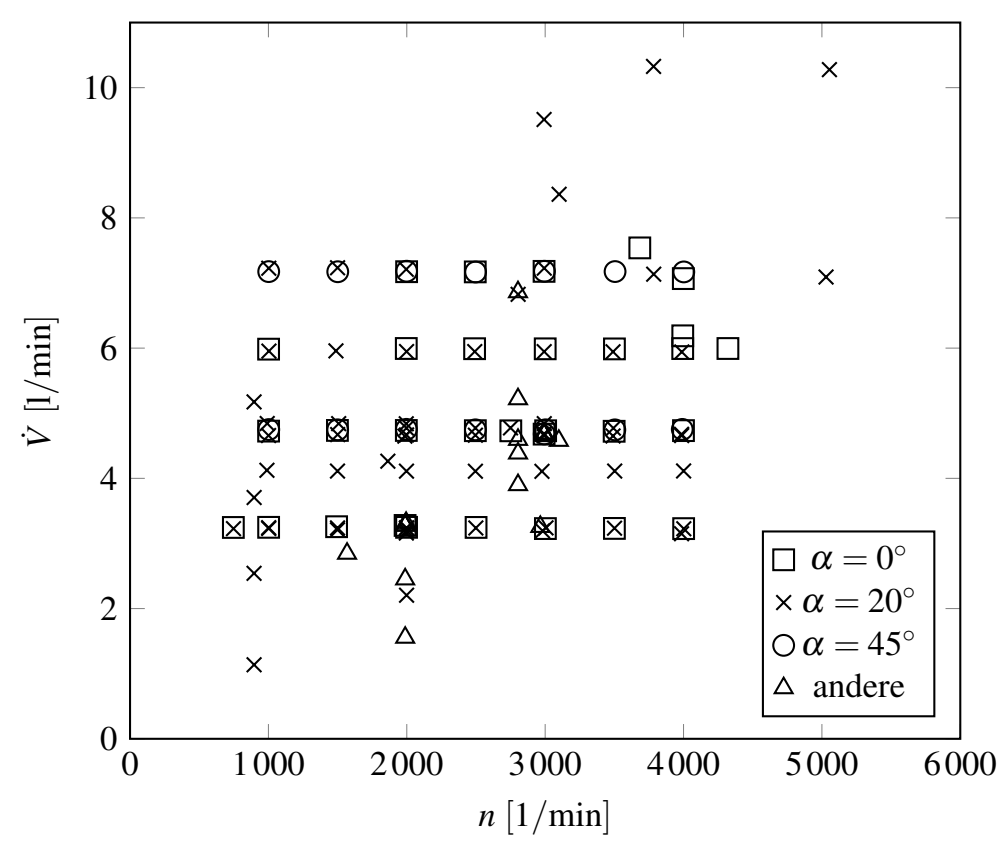

Abbildung 6.1: Verteilung der untersuchten Betriebspunkte im Parameterraum

Von insgesamt 129 Betriebspunkten entfallen 110 auf Düsenwinkel $\alpha$ von $0^{\circ}, 20^{\circ}$ und $45^{\circ}$. Für diese Düsenwinkel werden die Drehzahl $n$ in einem Bereich zwischen 1000/min und 4000/min 
und der Ölvolumenstrom $\dot{V}$ zwischen 3,21/min und 7,21/min systematisch variiert. Für $\alpha=20^{\circ}$ ist dieser Bereich am feinsten aufgelöst. Die Diskussion des Einflusses von Drehzahl und Ölvolumenstrom erfolgt für $\alpha=20^{\circ}$ in Abschnitt 6.2.1. Um den Einfluss des Düsenwinkels zu charakterisieren, werden in Abschnitt 6.2.2 die zusätzlichen Messungen bei $\alpha=45^{\circ}$ sowie Messungen bei weiteren Düsenwinkeln zwischen $-30^{\circ}$ und $45^{\circ}$ diskutiert. Eine vollständige Liste der Betriebspunkte und die ortsaufgelösten Darstellungen des Wärmeübergangs finden sich in Anhang A.1 dieser Arbeit.

\subsection{Messung und Auswertung des Wärmeübergangs}

Im Folgenden soll der Einfluss der variierten Parameter auf den Wärmeübergang beschrieben werden. Zunächst soll jedoch ein Betriebspunkt exemplarisch analysiert werden, um die Vorgehensweise zu verdeutlichen.

Alle gemessenen Größen werden während der Versuche aufgezeichnet, einige werden zur Regelung verwendet. Die Berechnung der Wärmeströme erfolgt auf Grundlage der so gewonnenen Daten.

Die benötigte Zeit, um das Öl, die Versorgungsanlage und den Prüfstand aufzuheizen und in einen stationären Zustand zu bringen, ist lang im Vergleich zu der Zeit, die zum Wechsel zwischen zwei Betriebspunkten benötigt wird. Da aus der Literatur nur wenige Informationen zur Beschreibung des Öl-Zahnrad-Wärmeübergangs zur Verfügung stehen, wird ein großer Bereich des Parameterraums durch Messungen abgedeckt, um alle wesentlichen Einflüsse aufzudecken (siehe Abbildung 6.1). Um die Erfassung der dafür benötigten großen Anzahl von Messpunkten effizient zu gestalten, werden mehrere Messungen in ähnlichen Parameterbereichen unmittelbar hintereinander durchgeführt. In Abbildung 6.2 sind die Verläufe der gemessenen Temperaturen, der Drehzahl und des Ölvolumenstroms über die Zeit für einen typischen Versuchsverlauf dargestellt. Die dargestellten Temperaturen im oberen Diagramm sind die des Öls in der Düse sowie ausgewählter Messpositionen an der Zahnradkontur und am Innenradius des Zahnrads.

Nach dem Beginn des Aufheizen des Öls bei $t \approx 0,3 \cdot 10^{3} \mathrm{~s}$ ist ein Ansteigen aller Temperaturen zu erkennen. Bei $t \approx 3 \cdot 10^{3}$ s erreicht die Öltemperatur den Sollwert von $80^{\circ} \mathrm{C}$. Zu diesem Zeitpunkt sind die Temperaturen des Zahnrads noch nicht stationär. Aufgrund der graduellen Änderung der sich einstellenden Temperaturen wird der Betriebspunkt gehalten, bis keine Änderungen der Temperaturen am Zahnrad und in der Öldüse mehr feststellbar sind, die über das Rauschen der Messwerte hinausgehen. Bei Erreichen des stationären Zustands wird dieser mindestens eine Minute gehalten, um eine ausreichende Datenbasis für die Mittelung zu erzeugen. Die letzten 30 Messwerte werden zur Mittelwertbildung herangezogen. Aufgrund der eingestellten Datenrate von $1 \mathrm{~Hz}$ entspricht dies einer Messung über einen Zeitraum von 30 Sekunden. Anschließend wird der nächste Parametersatz eingestellt und gehalten, bis erneut stationäre Zustände beobachtet werden. In Abbildung 6.2 ist dies durch den sequentiellen Wechsel der Kombinationen aus Drehzahl und Ölvolumenstrom und den sich jeweils einstellenden stationären Temperaturzuständen zu erkennen. Der Düsenwinkel bleibt in diesem Fall unverändert.

Am Verlauf der Öltemperatur in der Öldüse in Abbildung 6.2 ist erkennbar, dass die Öltemperatur 


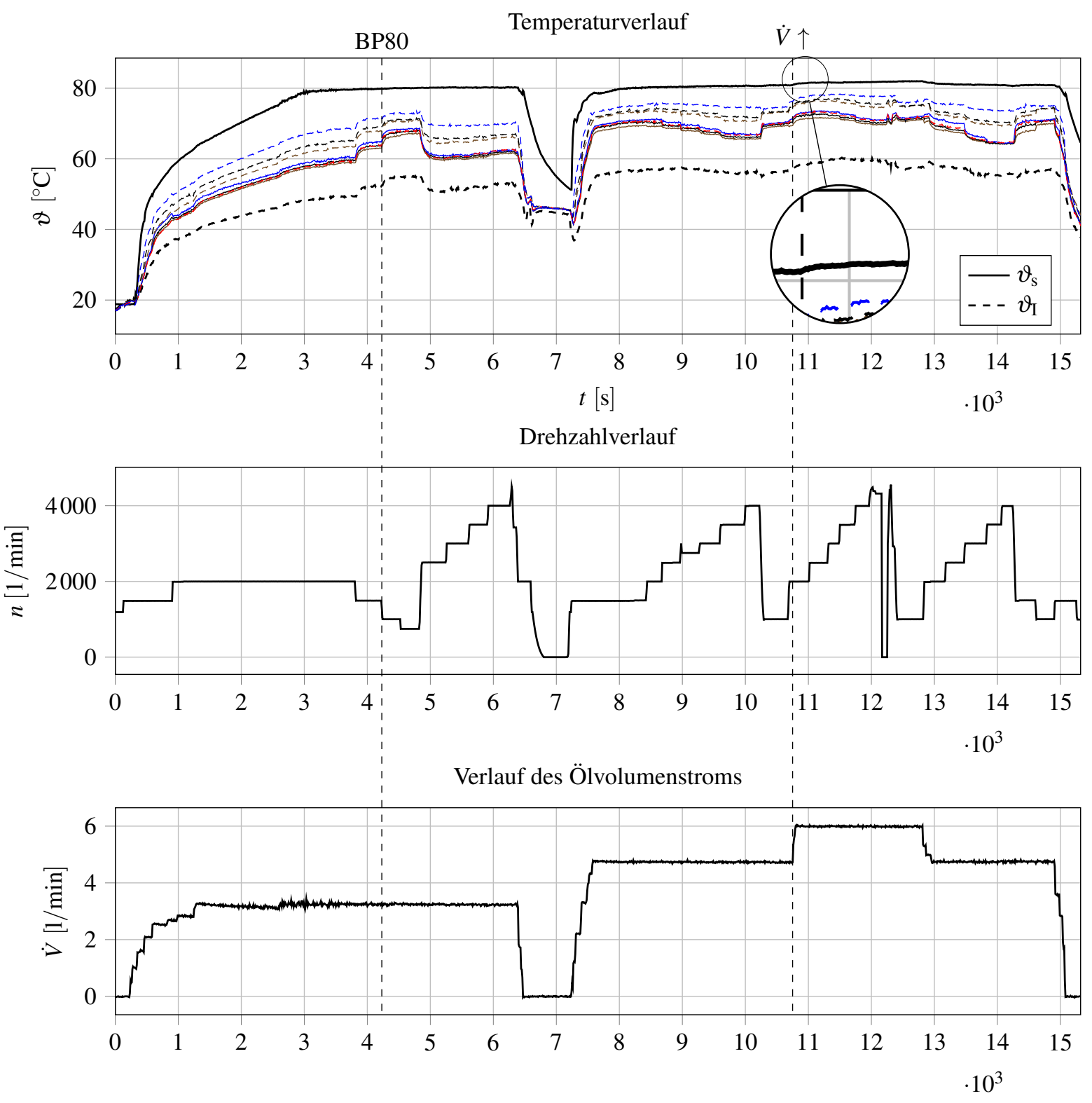

Abbildung 6.2: Exemplarischer Verlauf der Betriebsparameter und Temperaturen während einer Messung

in der Öldüse geringfügigen Schwankungen unterliegt. Diese Schwankungen treten vor allem bei einer Änderung des Ölvolumenstroms auf. Die Erhöhung des Ölvolumenstroms hat eine Erhöhung der Öltemperatur zur Folge, wie durch den vergrößerten Ausschnitt in Abbildung 6.2 verdeutlicht wird. Dies liegt daran, dass die Verweilzeit in der Zuleitung, die stellenweise nicht isoliert ist, bei höheren Ölvolumenströmen kürzer ist und somit das Öl zwischen Tank und Öldüse weniger abkühlt. Da die gemessene Öltemperatur in der Berechnung des Wärmeübergangs berücksichtigt wird und die Viskositätsänderung für die beobachteten Temperaturschwankungen sehr gering ist, kann dieser Effekt vernachlässigt werden, sofern die Öltemperatur stationäre Zustände erreicht.

Im Rahmen der exemplarischen Auswertung soll der Betriebspunkt 80 (BP80) betrachtet werden, 
dessen Messzeitpunkt in Abbildung 6.2 eingezeichnet und durch die in Tabelle 6.1 aufgelisteten Parameter bestimmt ist. Aufgrund des vergleichsweise langen Zeitraums der gesamten Messung im Vergleich zur Messung eines Betriebspunkts ist die Messung von BP80 zu einem Zeitpunkt dargestellt.

Tabelle 6.1: Parameter BP80

\begin{tabular}{lcr}
\hline Parameter & Sollwert & Istwert \\
\hline Volumenstrom & $3,21 / \mathrm{min}$ & $3,2571 / \mathrm{min}$ \\
Öltemperatur & $80^{\circ} \mathrm{C}$ & $79,87^{\circ} \mathrm{C}$ \\
Drehzahl & $1500 / \mathrm{min}$ & $1492 / \mathrm{min}$ \\
Düsenwinkel & $0^{\circ}$ & $-0,3^{\circ}$ \\
\hline
\end{tabular}
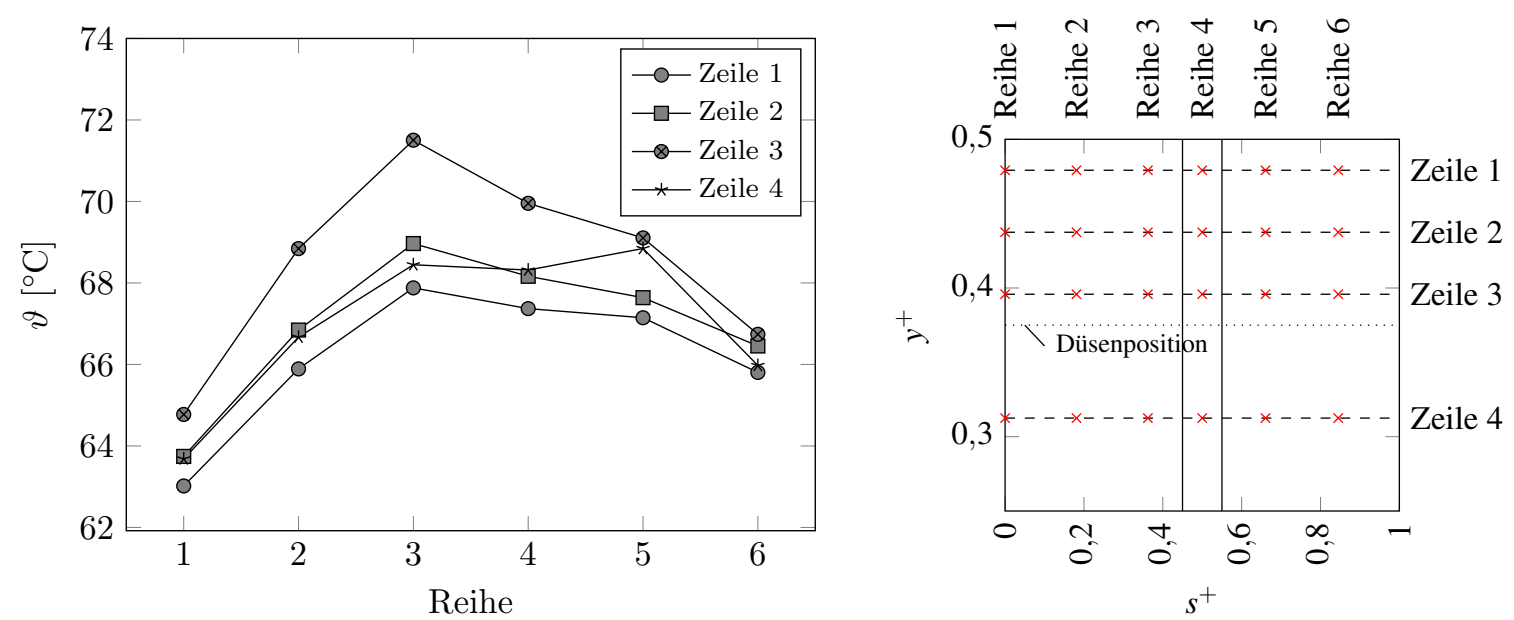

Abbildung 6.3: Gemessene Temperaturen auf der Zahnkontur für BP80

Für Betriebspunkt 80 wird die in Abbildung 6.3 dargestellte Temperaturverteilung gemessen, wobei die Temperaturen an Messpunkten gleicher axialer Position als Linien dargestellt sind. Der Temperaturverlauf entlang der Koordinate $s^{+}$zeigt für alle vier Messpunktzeilen einen qualitativ ähnlichen Verlauf. Die maximale Temperatur tritt für alle Zeilen an der Reihe 3, an der Position des größten Radius auf der strömungszugewandten Zahnflanke, auf. Eine Ausnahme bildet der Messpunkt 5 der Referenzmesszeile (Zeile 4), an dem geringfügig höhere Temperaturen gemessen werden als an Messpunkt 3. Das mittlere Temperaturniveau ist am höchsten für die Messreihe mit dem geringsten axialen Abstand zur Öldüse. Dies ist durch das dort aufprallende Öl und die damit verbundenen, hohen Wärmeübergänge zu begründen.

Zur Deutung der Abweichung des Messpunkt 5 der Referenzmesszeile kommen mehrere Ursachen in Frage. Eine Asymmetrie der Wärmeübergangsverteilung ist grundsätzlich denkbar. Allerdings wird eine deutliche Temperaturabweichung nur an diesem Messpunkt beobachtet. In Messungen bei anderen Betriebspunkten werden ebenfalls Temperaturabweichungen beobachtet, diese fallen jedoch meist geringer aus. Des weiteren besteht die Möglichkeit, dass das Thermoelement dieser Messstelle eine Abweichung in seiner Einbettungstiefe ausweist. Eine solche 
Abweichung ist jedoch allenfalls in einem geringen Maße denkbar, da die Thermoelementnut nur geringfügig größer ist als der Durchmesser des Thermoelements und ein Herausragen des Thermoelements deutlich sichtbar wäre. Dies lässt entweder eine herstellbedingte Abweichung im verwendeten Thermoelement oder in der Signalkette der Telemetrie als wahrscheinlichere Ursache erscheinen.

Um aus den gemittelten Temperaturdaten zunächst Wärmeströme im Testzahnrad und anschließend, mit bekannter Öltemperatur, Wärmeübergänge zu berechnen, wird das in Kapitel 4 beschriebene iterative Verfahren angewendet. Dabei werden die Messwerte auf einem Achtelzahn, der Hälfte eines periodischen Segments, zur Berechnung verwendet. Da die Interpretation eines ganzen Segments jedoch wesentlich anschaulicher ist, wird in allen folgenden Darstellungen die Wärmeübergangsverteilung entlang der Mitte eines Segments gespiegelt und zwischen $y^{+}=0,25$ und $y^{+}=0,5$ betrachtet. Die zweidimensionale Verteilung des Wärmeübergangs für BP80 ist in Abbildung 6.4 dargestellt.

Wie aus der Interpretation der Temperaturen bereits vermutet, konzentriert sich das Wärmeübergangsmaximum an der axialen Position der Düse und an der strömungszugewandten Flanke. Dort werden Werte im Bereich von $1000 \mathrm{~W} /\left(\mathrm{m}^{2} \mathrm{~K}\right)$ erreicht. Entlang der Symmetrielinie $y^{+}=0,375$ nimmt der Wärmeübergang zum Zahnfuß hin ab und erreicht dort Werte von ca. $600 \mathrm{~W} /\left(\mathrm{m}^{2} \mathrm{~K}\right)$. Auf der strömungsabgewandten Zahnflanke werden insgesamt geringere Werte des Wärmeübergangs gemessen, dessen Anteil am gesamten Wärmeübergang jedoch nicht zu vernachlässigen ist. Der Wärmeübergang in axialer Richtung ist gleichmäßiger verteilt als auf der strömungszugewandten Zahnflanke. Dies kann ein Hinweis auf das Abprallen des Strahls sein, das von Keller et al. (2017) beobachtet wurde.

Negative Werte für $h$ werden in flächenmäßig kleinen Bereichen in der Nähe des Zahnkopfes, außerhalb der Mitte beobachtet. Das Auftreten negativer Wärmeübergänge ist unphysikalisch, da diese einen Wärmestrom in Richtung eines positiven Temperaturgradienten erfordern und somit den zweiten Hauptsatz der Thermodynamik verletzen würden. Im Fall der vorliegenden Untersuchungen ist die Ursache dieser negativen Wärmeströme jedoch in der vergleichsweise geringen Ortsauflösung der Messpunkte zu suchen. Bereiche geringen Wärmestroms werden durch die Interpolation unter Umständen überschätzt. Aus Wärmebilanzgründen ergibt sich dadurch an anderen Stellen ein negativer Wärmeübergang. Darüber hinaus wird durch die verwendete Definition des Wärmeübergangs der Einfluss der im Prüfstand vorhandenen Luft nicht berücksichtigt. Durch Abschätzungen konnte gezeigt werden, dass dieser Einfluss im Vergleich zur Prallkühlung gering ist. Jedoch besteht die Möglichkeit, dass die Luft durch Abkühlung an den metallischen Wänden des Prüfstands eine geringere Temperatur aufweist als das Öl. Wenn das Öl die strömungsabgewandte Flanke nicht erreicht und somit keinen Wärmestrom in den Zahn hervorruft, kann der Wärmeübergang durch Luft, der zu einer Wärmeabfuhr führt, zur Berechnung von negativen Wärmeübergangskoeffizienten führen. 


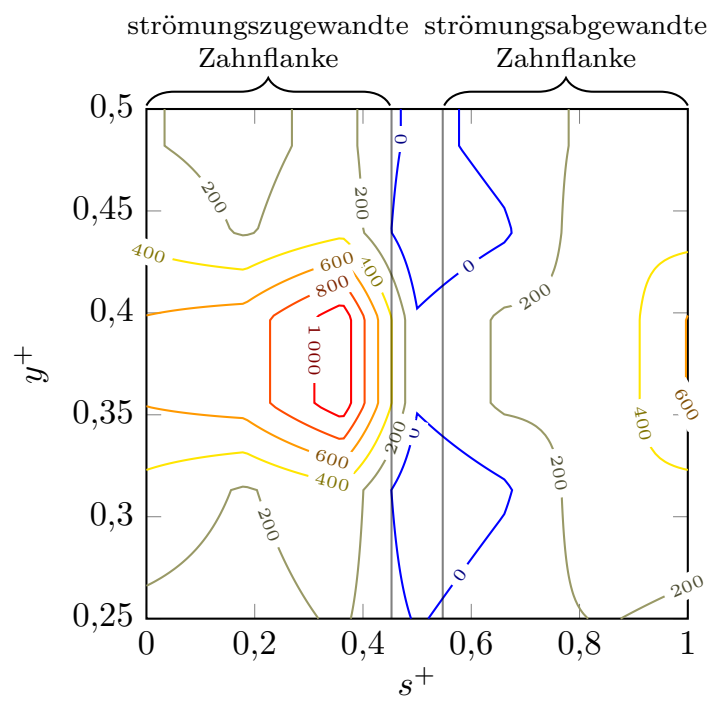

Abbildung 6.4: Wärmeübergangsverteilung für BP80 in $\mathrm{W} /\left(\mathrm{m}^{2} \mathrm{~K}\right)$

\subsection{Charakterisierung des Öl-Zahnrad-Wärmeübergangs}

Ziel der vorliegenden Arbeit ist es, den Wärmeübergang an einem einspritzgekühlten Zahnrad experimentell zu ermitteln und den Einfluss der Betriebsparameter auf den Wärmeübergang zu quantifizieren. Die im Vorfeld bekannten Zusammenhänge und das verfügbare Wissen zur Öl-Zahnrad-Interaktion sind äußerst begrenzt. Die häufig herangezogene Wärmeübergangskorrelation von DeWinter und Blok (1974) bildet einzig die Drehzahl als Einflussgröße auf den Wärmeübergang ab. Aus Arbeiten von Townsend und Akin (1981), Long et al. (2003) und weiteren ist jedoch bekannt, dass der Ölvolumenstrom einen erheblichen Einfluss auf den Wärmeübergang hat.

Um sämtliche Einflüsse der mit dem verwendeten Prüfstand variierbaren Parameter zu erfassen, wird eine Vielzahl von Messungen unterschiedlicher Betriebspunkte vorgenommen. Aus Gründen der Nachvollziehbarkeit sollen die jeweiligen Parameter nacheinander diskutiert werden. Dabei können nur einige Messungen detailliert betrachtet werden. Für eine eingehende Darstellung aller Einzelergebnisse sei daher auf den Anhang verwiesen.

Zunächst soll in Abschnitt 6.2.1 der Einfluss der Drehzahl und des Ölvolumenstroms auf den Wärmeübergang diskutiert werden. Anschließend wird in Abschnitt 6.2.2 die Abhängigkeit des Wärmeübergangs vom Düsenwinkel betrachtet. Die diskutierten Messungen in diesen beiden Abschnitten beziehen sich dabei stets auf Öltemperaturen von $80^{\circ} \mathrm{C}$. Da bei geänderten Temperaturen und somit geänderten Prandtlzahlen auch mit geänderten Strömungsvorgängen und Wärmeübergängen zu rechnen ist, wird der Einfluss der Prandtlzahl in Abschnitt 6.2.3 ebenfalls untersucht.

Um die charakteristischen Abhängigkeit des Wärmeübergangs von den Betriebsparametern in einer möglichst allgemeinen Form zu analysieren, werden die dimensionslosen Größen Geschwindigkeitsverhältnis $\Sigma$, Eindringwinkel $\beta$ und die Relativgeschwindigkeit $u_{\mathrm{r}}$ herangezogen. Aus der Analyse im Folgenden wird deutlich, dass die wesentlichen Wirkzusammenhänge durch 
diese Größen erfasst werden.

Die Beschreibung des charakteristischen Verhaltens des Wärmeübergangs alleine durch diese drei Größen greift jedoch zu kurz. Die Drehzahl und der Ölvolumenstrom wirken sich auf eine Art auf die gemessenen Wärmeströme aus, die über ihre Bedeutung beispielsweise für $\Sigma$ hinausgeht. Wie in Abschnitt 3.2 beschrieben, ist zum einen zwischen anliegender und abgelöster Strömung zu unterscheiden. Außerdem beeinflusst die Drehzahl das Strömungsfeld um das Zahnrad. Daher werden diese beiden Größen ebenfalls in der folgenden Analyse herangezogen. Der Ölvolumenstrom wird durch die Strahlreynoldszahl

$$
\operatorname{Re}_{d}=\frac{\rho u_{\mathrm{s}} d}{\mu}=\frac{u_{\mathrm{s}} d}{v}
$$

beschrieben. Die Drehzahl wird durch die Rotationsreynoldszahl

$$
\operatorname{Re}_{\omega}=\frac{\omega D^{2}}{v}
$$

ausgedrückt. Als charakteristischer Durchmesser $D$ wird dabei der Kopfkreisdurchmesser verwendet.

Die verwendete Methode zur Messung des Öl-Zahnrad-Wärmeübergangs liefert ortsaufgelöste Daten. Um den Einfluss einzelner Parameter auf den Wärmeübergang zu charakterisieren, ist es sinnvoll, auch den gemittelten Wärmeübergang zu betrachten, als

$$
\overline{\mathrm{Nu}}=\frac{1}{\mathrm{Nu}^{*}} \frac{1}{0,25} \int_{0}^{1} \int_{0,25}^{0,5} \mathrm{Nu}\left(s^{+}, y^{+}\right) d y^{+} d s^{+},
$$

mit dem Normierungsfaktor $\mathrm{Nu}^{*}$, der der höchsten im Rahmen dieser Arbeit gemessenen mittleren Nußeltzahl entspricht.

\subsubsection{Einfluss der Drehzahl und des Ölvolumenstroms}

Um den Wärmeübergang am Zahnrad zu charakterisieren, soll zunächst der in Messungen beobachtete Einfluss der Drehzahl $\left(\operatorname{Re}_{\omega}\right)$ und des Ölvolumenstroms $\left(\operatorname{Re}_{d}\right)$ auf die Nußeltzahlverteilung betrachtet werden. In Abbildung 6.5 ist der Verlauf von $\overline{\mathrm{Nu}} *$ bei $\operatorname{Re}_{d}=2639$ und $\alpha=20^{\circ}$ über einen Bereich von $\operatorname{Re}_{\omega}$ zwischen $48 \cdot 10^{4}$ und $190 \cdot 10^{4}$ dargestellt. Zusätzlich sind das Geschwindigkeitsverhältnis $\Sigma$ und der Aufprallwinkel $\beta$ über den betrachteten Bereich eingezeichnet. Durch die Beschreibung und Diskussion der vier markierten Betriebspunkte von niedrigen zu hohen Drehzahlen soll der Verlauf von $\overline{\mathrm{Nu}} *$ im Folgenden erklärt werden. Dazu werden ergänzend Einzelbilder der Gegenlichtaufnahmen in Abbildung 6.6 herangezogen.

In Abbildung 6.5 sind für zwei Betriebspunkte Wiederholmessungen verfügbar. Diese weisen

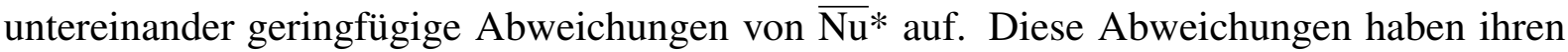
Ursprung in versuchsbedingten Einflüssen, wie beispielsweise leicht unterschiedliche Ölvorlauftemperaturen durch Messungen zu unterschiedlichen Zeitpunkten. Für eine besser lesbare Darstellung des Kurvenverlaufs wird der interpolierte Verlauf von $\overline{\mathrm{Nu}^{*}}$ als Linie dargestellt. An 


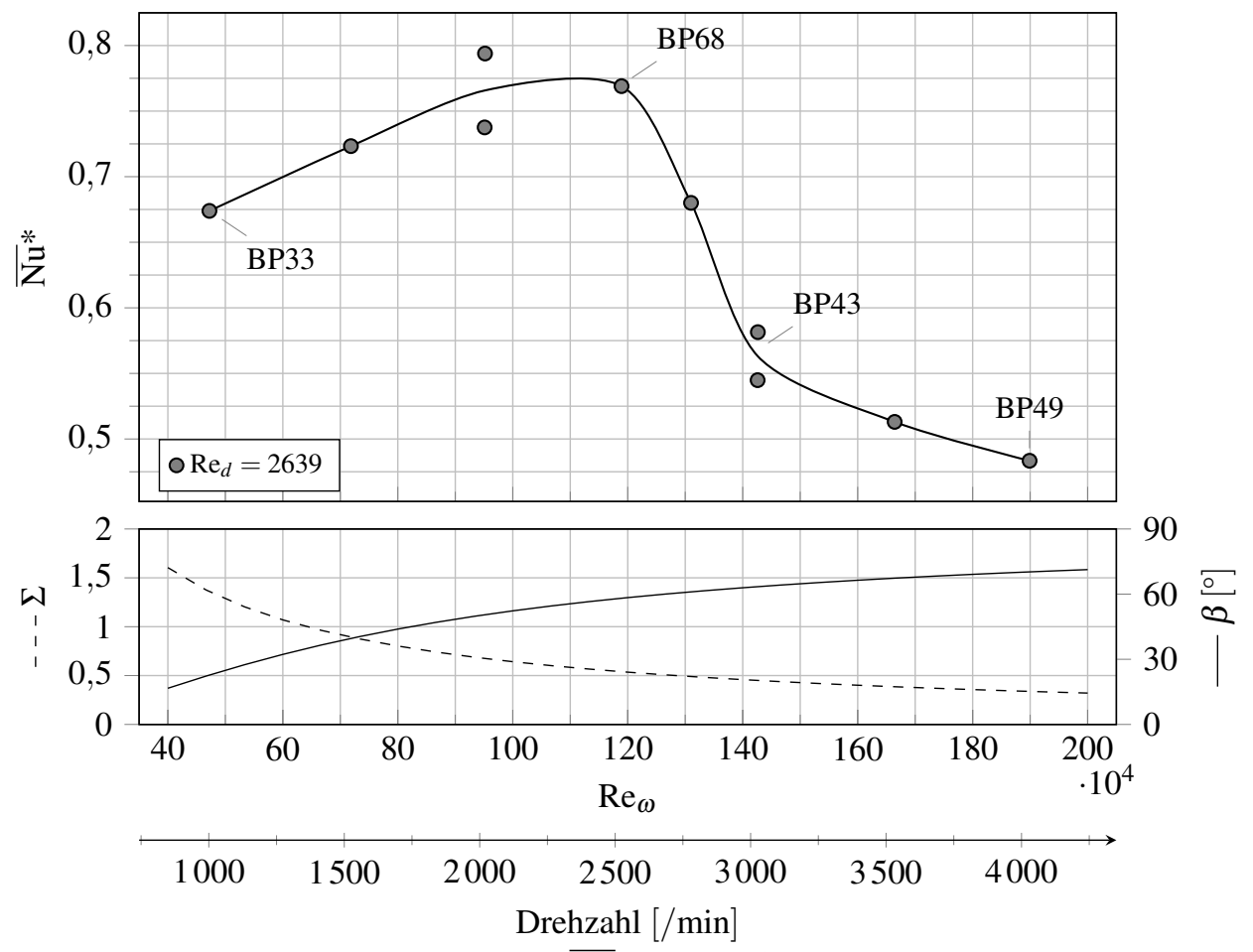

(a) Verlauf von $\overline{\mathrm{Nu}}^{*}, \Sigma$ und $\beta$ über $\operatorname{Re}_{\omega}$

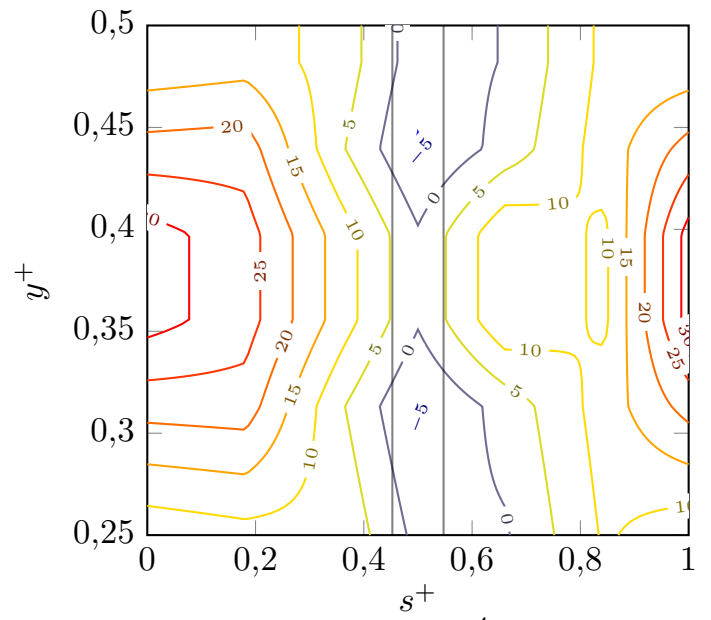

(b) $\mathrm{BP} 33, \operatorname{Re}_{\omega}=48 \cdot 10^{4}, \beta=22,49^{\circ}$

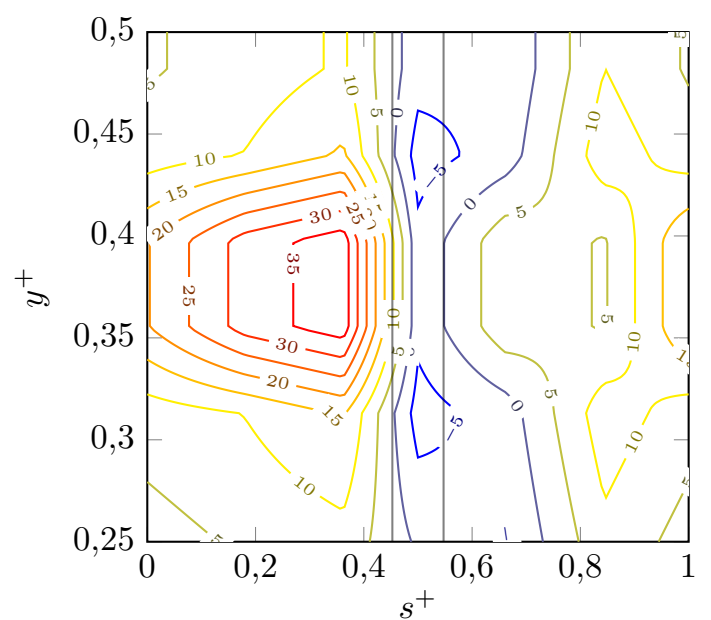

(d) BP43, $\operatorname{Re}_{\omega}=143 \cdot 10^{4}, \beta=63,8^{\circ}$

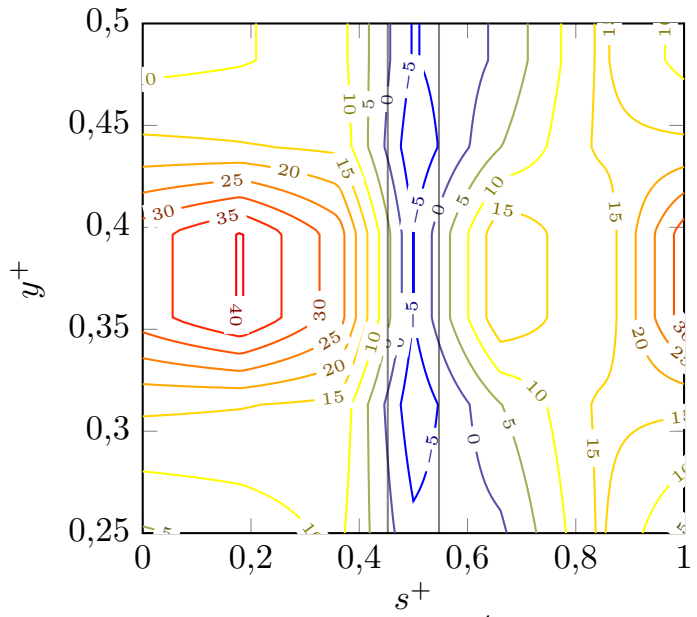

(c) $\mathrm{BP} 68, \mathrm{Re}_{\omega}=119 \cdot 10^{4}, \beta=57,95^{\circ}$

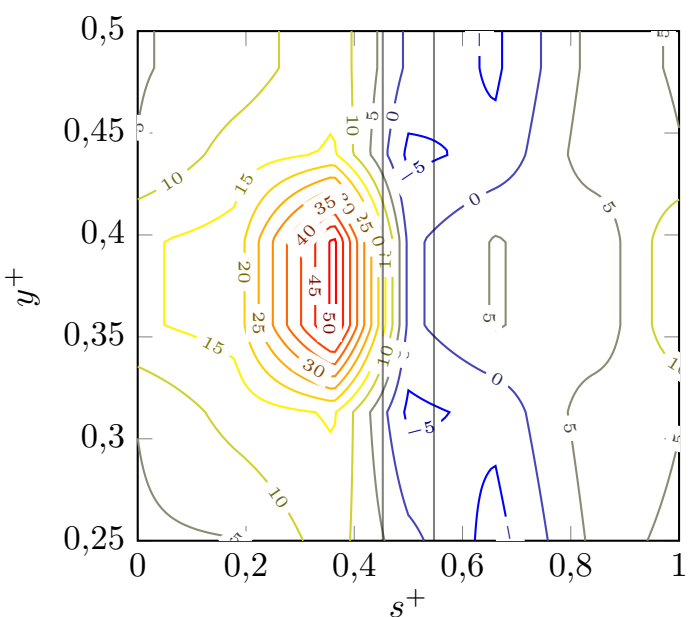

(e) $\mathrm{BP} 49, \operatorname{Re}_{\omega}=191 \cdot 10^{4}, \beta=70,55^{\circ}$

Abbildung 6.5: Einfluss der Drehzahl $\mathrm{Re}_{\omega}$ auf den flächengemittelten Wärmeübergang $\overline{\mathrm{Nu}}^{*}$ bei $\mathrm{Re}_{d}=2639$ und den ortsaufgelösten Wärmeübergang $\mathrm{Nu}$ 


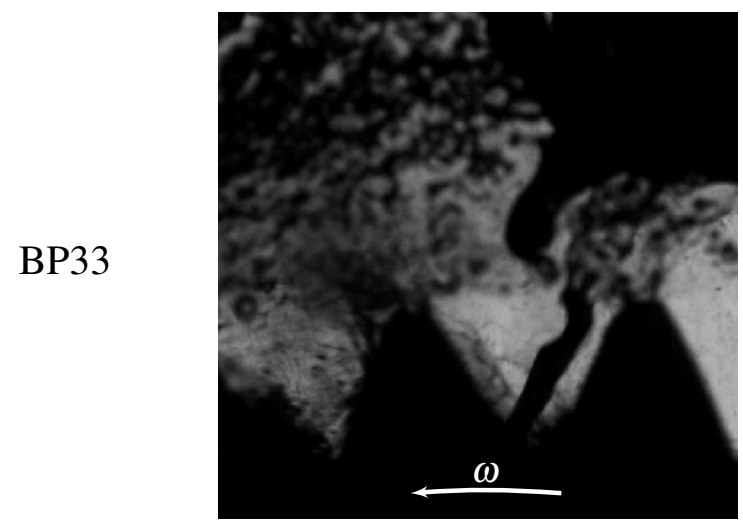

(a) $\operatorname{Re}_{\omega}=48 \cdot 10^{4}$

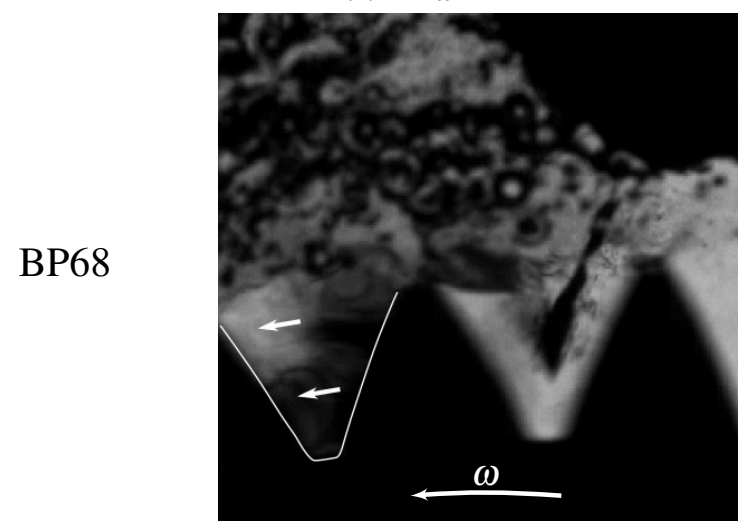

(c) $\operatorname{Re}_{\omega}=119 \cdot 10^{4}$

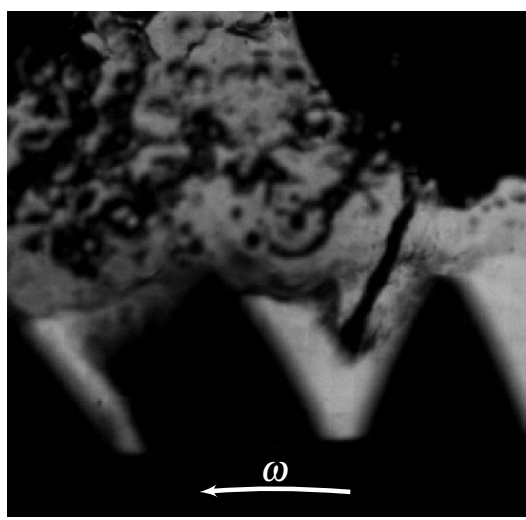

(e) $\operatorname{Re}_{\omega}=143 \cdot 10^{4}$

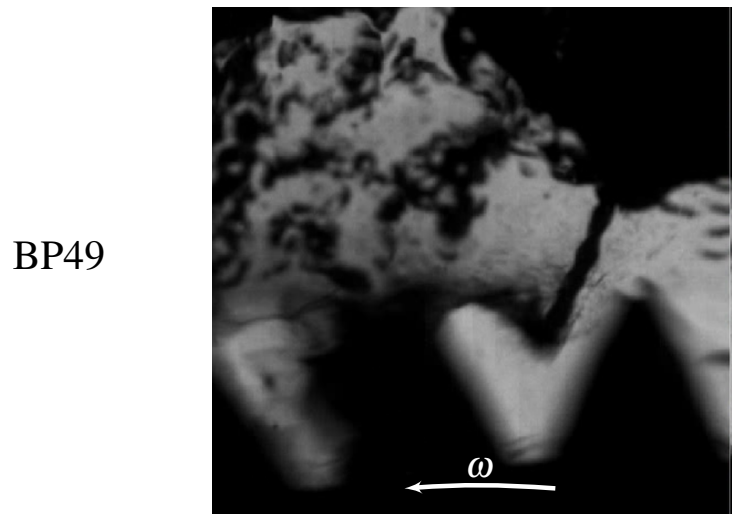

(g) $\operatorname{Re}_{\omega}=191 \cdot 10^{4}$

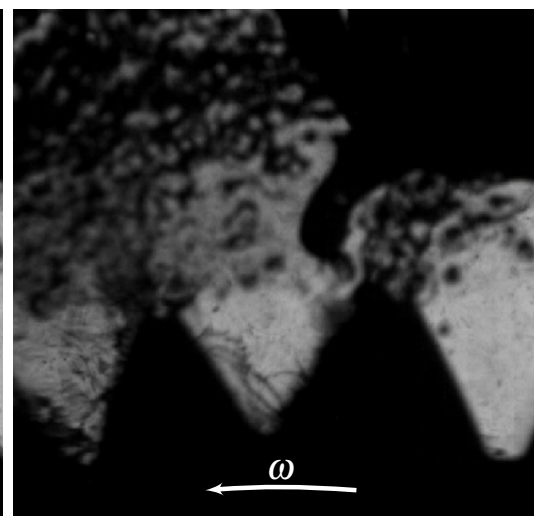

(b) $\operatorname{Re}_{\omega}=48 \cdot 10^{4}$

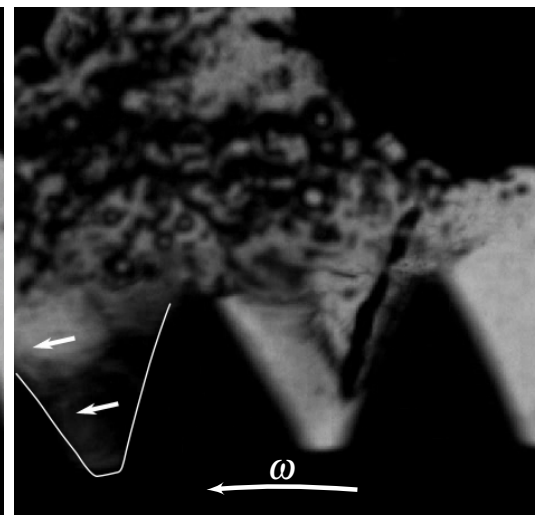

(d) $\operatorname{Re}_{\omega}=119 \cdot 10^{4}$

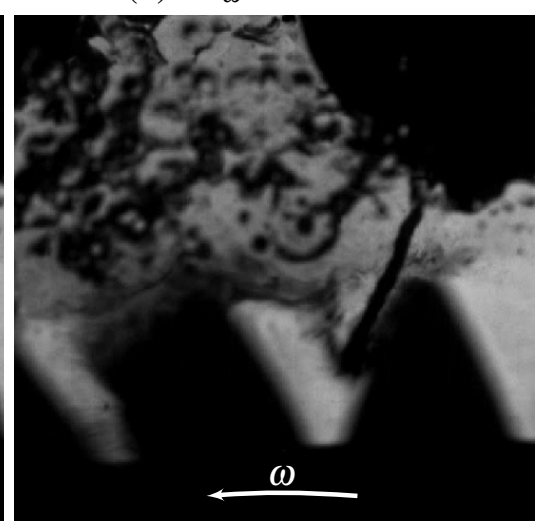

(f) $\operatorname{Re}_{\omega}=143 \cdot 10^{4}$

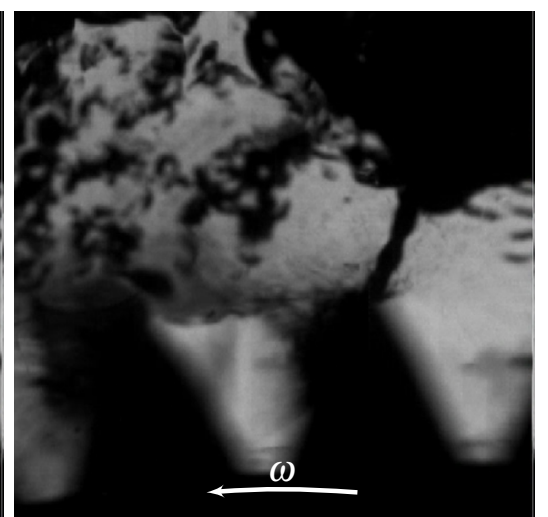

(h) $\operatorname{Re}_{\omega}=191 \cdot 10^{4}$

Abbildung 6.6: Gegenlichtaufnahmen bei $\operatorname{Re}_{d}=2639$ 
Betriebspunkten, an denen mehrere Messungen vorliegen, wird der Mittelwert aller verfügbarer Messungen als Grundlage für die Interpolation herangezogen.

Zwei Einzelbilder der Gegenlichtaufnahme bei $\operatorname{Re}_{\omega}=48 \cdot 10^{4}$ (BP33) sind in Abbildungen 6.6a und $6.6 \mathrm{~b}$ gezeigt. Bedingt durch das vergleichsweise hohe Geschwindigkeitsverhältnis mit $\Sigma=1,4$ und den positiven Düsenwinkel von $\alpha=20^{\circ}$ ergibt sich ein steiler Einfall des Ölstrahls mit einem Aufprallwinkel $\beta$ von 22,49 ${ }^{\circ}$. Dies ist daran zu erkennen, dass der Ölstrahl zu einem Zeitpunkt kurz vor dem Erreichen der strömungszugewandten Flanke bereits den Zahnfuß erreicht hat. Das untere Drittel der Zahnlücke ist zu diesem Zeitpunkt mit Öl gefüllt. Der ortsaufgelöste Wärmeübergang bei $\operatorname{Re}_{\omega}=48 \cdot 10^{4}$ (BP33) ist in Abbildung 6.5b dargestellt. Es wird eine Wärmeübergangsverteilung gemessen, die am Zahnfuß konzentriert ist und somit die Beobachtungen aus den Gegenlichtaufnahmen bestätigt. Dort werden lokale Nußeltzahlen von etwa 30 beobachtet. Dies kann auf das steile Eindringen des Ölstrahls und das dadurch hervorgerufene Auftreffen des Öls am Zahnfuß begründet werden. In lateraler Richtung werden in der Nähe der Position der Düse, $y^{+}=0,375$, höhere Wärmeübergangskoeffizienten als an den Rändern des periodischen Elements gemessen. Auf der strömungszugewandten Zahnflanke sind höhere Wärmeübergangskoeffizienten zu beobachten als auf der gegenüberliegenden, strömungsabgewandten Flanke. Die Ursache für diesen Unterschied kann in der höheren Menge Öl gesehen werden, die aufgrund der kinematischen Verhältnisse auf der strömungszugewandten Zahnflanke auftrifft. Im Bereich des Zahnkopfes wird das geringste Niveau des Wärmeübergangs beobachtet. Stellenweise treten hier leicht negative Werte auf.

Bei Erhöhung der Drehzahl (siehe Verlauf in Abbildung 6.5a) nimmt einerseits das Verhältnis aus Strahl- und Tangentialgeschwindigkeit $\Sigma$ stetig ab, während der Aufprallwinkel $\beta$ zunimmt (siehe Abbildung 3.5). Dies ist gleichbedeutend mit einem flacheren Auftreffen des Ölstrahls auf die Zahnoberfläche und einer geringeren radialen Eindringtiefe. Andererseits ist durch den erhöhten spezifischen Impuls des auftreffenden Öls mit erhöhtem Wärmeübergang zu rechnen. Ausgehend von $\operatorname{Re}_{\omega}=48 \cdot 10^{4}$ (BP33) ist bei Erhöhung der Drehzahl zunächst ein Anstieg von $\overline{\mathrm{Nu}}^{*} \mathrm{zu}$ beobachten. In diesem Bereich überwiegt demnach der Effekt des erhöhten Impulses. Bei $\operatorname{Re}_{\omega}=118 \cdot 10^{4}$ (BP68, Abbildung 6.5c) ist das örtliche Maximum des Wärmeübergangs durch die Änderung der kinematischen Verhältnisse in Richtung des Zahnkopfes und in positive $s^{+}$-Richtung verschoben und mit etwa $\mathrm{Nu}=40$ deutlich höher. Aus den Gegenlichtaufnahmen in Abbildungen 6.6c und 6.6d ist ersichtlich, dass der Ölstrahl an diesem Betriebspunkt flacher in die Zahnlücke eindringt und den Zahnfuß nicht erreicht. In lateraler Richtung ist, ausgehend vom Bereich des Maximums auf der strömungszugewandten Seite, ein starker Abfall des Wärmeübergangs zu den Rändern des Bereichs zu beobachten. Auf der unbelasteten Zahnflanke ist jedoch ein Bereich bei etwa $s^{+}=0,8$ zu erkennen, an dem entlang des gesamten $y^{+}$-Bereichs nahezu konstante Nußeltzahlen gemessen werden. Dies ist möglicherweise auf das Abprallen des Ölstrahls an der Aufprallstelle, anschließendes Auffächern und erneutes Auftreffen auf der strömungsabgewandten Seite zurückzuführen. In den Abbildungen 6.6c und 6.6d ist zur Veranschaulichung dieses Zusammenhangs die Zahnkontur der linken benachbarten Zahnlücke hervorgehoben. Hier ist zu erkennen, dass das Öl etwa die untere Hälfte der Zahnlücke füllt (Abb. 6.6c) und sich auch in der oberen Hälfte auf die strömungsabgewandte Zahnflanke zubewegt (Abb. 6.6c), wie durch die beiden Pfeile angedeutet. 


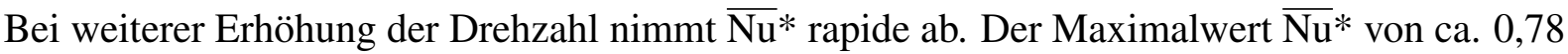
des mittleren Wärmeübergangs für $\operatorname{Re}_{d}=2639$ und $\alpha=20^{\circ}$ bei $\operatorname{Re}_{\omega} \approx 118 \cdot 10^{4}$ (BP68) sinkt auf etwa 0,57 bei $\operatorname{Re}_{\omega}=143 \cdot 10^{4}$ (BP43). In Abbildung 6.5d ist zu erkennen, dass das Maximum des Wärmeübergangs weiter in Richtung des Zahnkopfes verschoben ist. Sowohl das Niveau als auch die örtliche Ausdehnung des Bereichs hohen Wärmeübergangs auf der strömungszugewandten Seite sind vergleichbar mit der Verteilung des Wärmeübergangs für BP68 in Abbildung 6.5c. Auf der strömungsabgewandten Zahflanke wird jedoch ein deutlicher Unterschied der Verteilungen gemessen. In der Nähe des Zahnkopfes ist der Wärmeübergang auf etwa ein Drittel des Wertes von BP68 reduziert, in weiten Bereichen des Zahnfußes ist eine Reduktion auf etwa die Hälfte zu beobachten. Als mögliche Erklärung können Abbildungen 6.6e und 6.6f herangezogen werden. Dort ist ein ölfreier Spalt an der strömungsabgewandten Zahnflanke zu erkennen, während bei BP68 beobachtet wurde, dass Öl die strömungsabgewandte Zahnflanke erreicht (vgl. Abb. 6.6c und 6.6d). Durch das Abreißen der Ölzufuhr an die strömungsabgewandte Zahnflanke kann hier auch keine Kühlwirkung hervorgerufen werden.

Bei Erhöhung der Drehzahl auf $\operatorname{Re}_{\omega}=191 \cdot 10^{4}$ (BP49) setzt sich die Verschiebung des Bereichs hohen Wärmeübergangs in Richtung des Zahnkopfes fort. Dieser ist nun stark in der Nähe des Zahnkopfes auf der strömungszugewandten Seite konzentriert (siehe Abbildung 6.5e). Durch die Erhöhung des Impulses ist auch das lokale Maximum des Wärmeübergangs erhöht. Während dort Maximalwerte von etwa $\mathrm{Nu}=50$ gemessen werden, ist auf der strömungsabgewandten Seite der Wärmeübergang zum Erliegen gekommen. Diese Beobachtungen stellen eine Fortsetzung der zuvor beobachteten Effekte dar. Aus Abbildungen 6.6g und 6.6h ist ersichtlich, dass der Ölstrahl bei BP49 noch weniger tief in die Zahnlücke eindringt und dass die strömungsabgewandte Zahnflanke nahezu frei von Öl bleibt.

Analog zur vorangegangenen Betrachtung und Abbildung 6.5 soll im Folgenden der drehzahl-

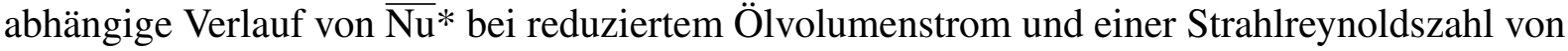
$\operatorname{Re}_{d}=1757$ diskutiert werden. Dazu werden der Verlauf des mittleren Wärmeübergangs und die Wärmeübergangsverteilungen an vier Betriebspunkten für $\mathrm{Re}_{d}=1757$ in Abbildung 6.7 betrachtet. Insgesamt liegt der Wärmeübergang auf einem geringeren Niveau. Während bei $\operatorname{Re}_{d}=2639$ und $\operatorname{Re}_{\omega}=118 \cdot 10^{4}$ maximale Werte von $\overline{\mathrm{Nu}}^{*} \approx 0,78$ erreicht werden, werden bei $\operatorname{Re}_{d}=1757$ lediglich mittlere Nußeltzahlen von ca. 0,6 bei $\operatorname{Re}_{\omega} \approx 40 \cdot 10^{4}$ gemessen.

Durch die geringere Strahlgeschwindigkeit bei $\operatorname{Re}_{d}=1757$ ist der Aufprallwinkel $\beta$ bei gleichen Werten für $\operatorname{Re}_{\omega}$ stets höher als in der vorangegangen Betrachtung bei $\operatorname{Re}_{d}=2639$. Dies äußert sich in einem Abfall von $\overline{\mathrm{Nu}}{ }^{*}$ bei geringeren Drehzahlen, welcher zwischen $\operatorname{Re}_{\omega}=48 \cdot 10^{4}$ und $\operatorname{Re}_{\omega}=95 \cdot 10^{4}$ beobachtet wird.

In Abbildung 6.7b ist der ortsaufgelöste Wärmeübergang $\operatorname{Re}_{\omega}=48 \cdot 10^{4}$ (BP53) dargestellt. Der Aufprallwinkel an diesem Betriebspunkt ist mit $\beta=38,44^{\circ} \mathrm{klein}$, es ist mit einem steilen Einfall des Ölstrahls zu rechnen. Dies führt zu einer Konzentration des Wärmeübergangs am Zahnfuß, wie sie auch bei $\operatorname{Re}_{d}=2639$ und kleinen Aufprallwinkeln beobachtet wird.

Bei Erhöhung der Drehzahl auf $\operatorname{Re}_{\omega}=71 \cdot 10^{4}$ (BP29, siehe Abb. 6.7a) und einem daraus resultierenden Aufprallwinkel von $\beta=54,32^{\circ}$ wird die in Abbildung 6.7c dargestellte Wärmeübergangsverteilung gemessen. Entlang der Linie der axialen Düsenposition, $y^{+}=0,375$, 

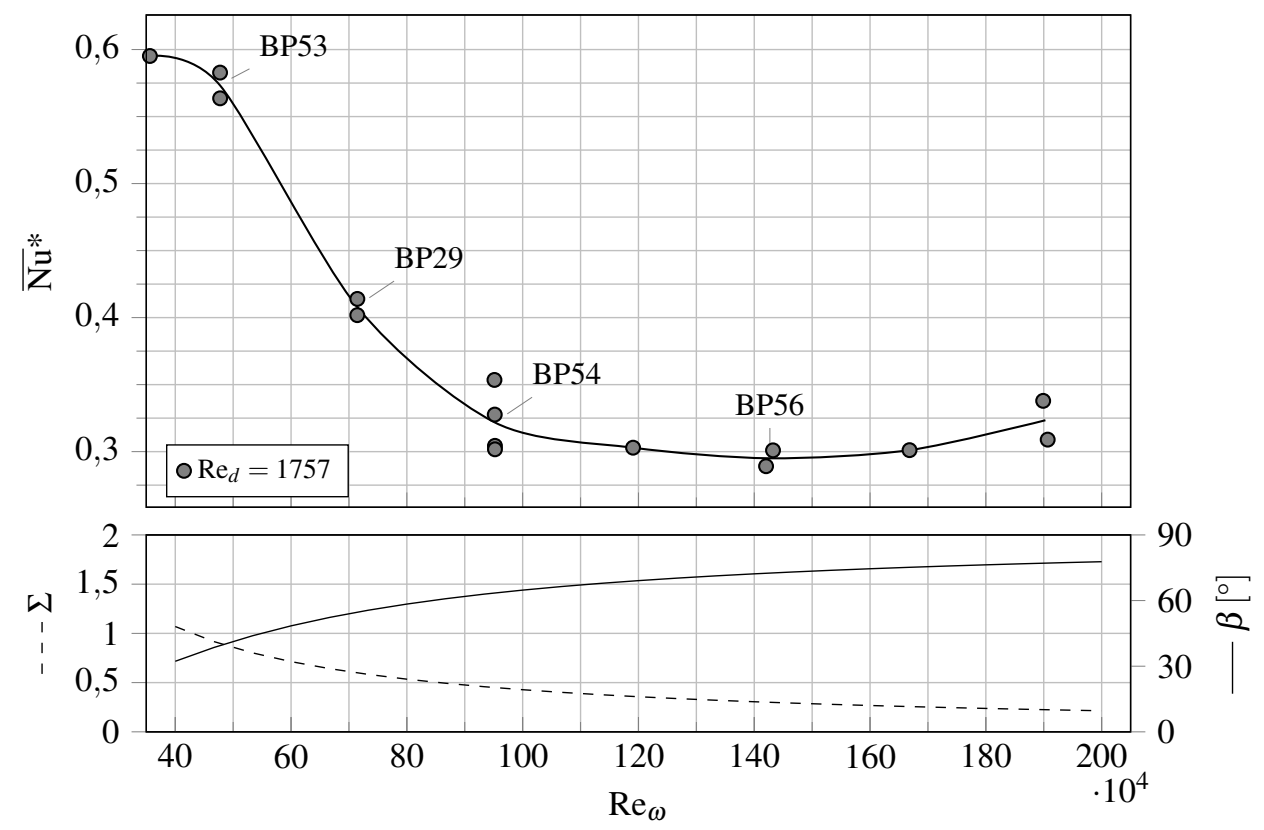

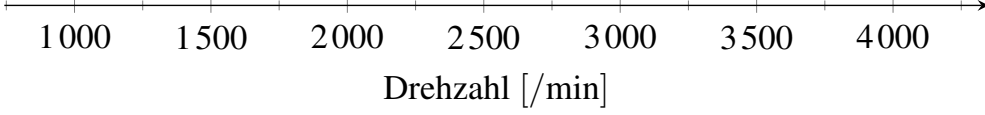

(a) Verlauf von $\overline{\mathrm{Nu}}^{*}, \Sigma$ und $\beta$ über $\operatorname{Re}_{\omega}$

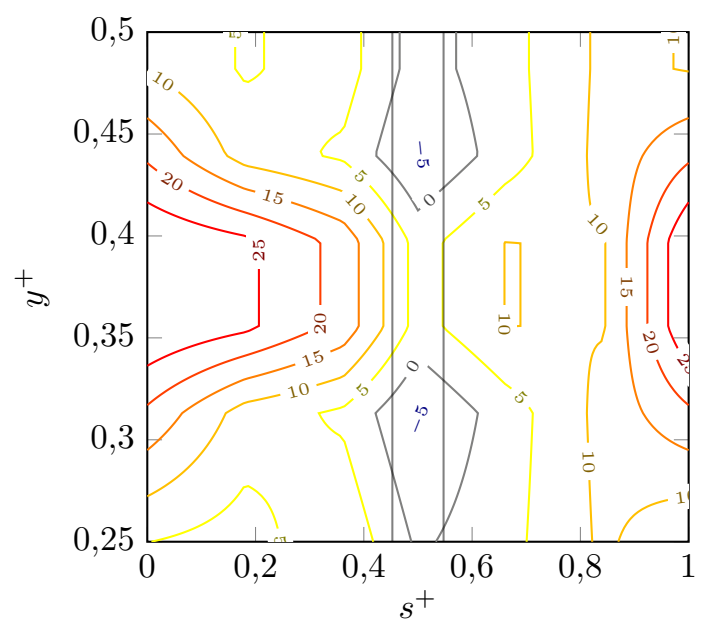

(b) BP53, $\operatorname{Re}_{\omega}=48 \cdot 10^{4}, \beta=38,44^{\circ}$

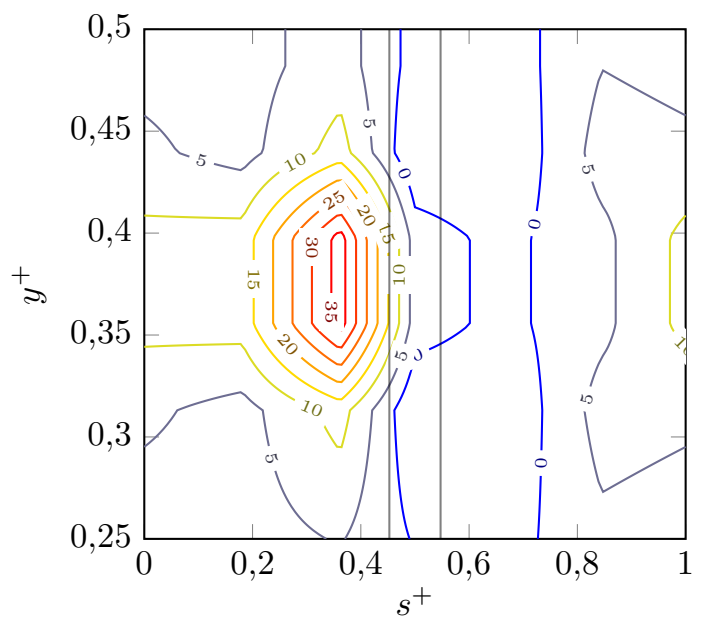

(d) BP54, $\operatorname{Re}_{\omega}=95 \cdot 10^{4}, \beta=62,89^{\circ}$

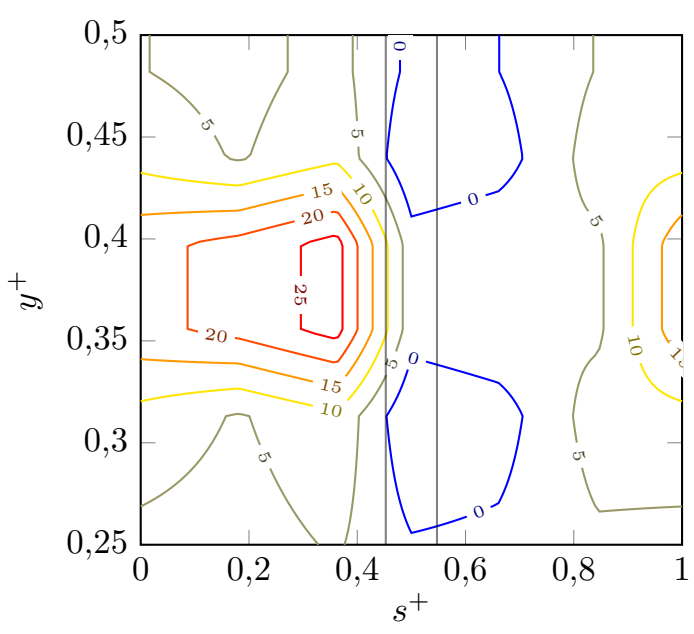

(c) $\mathrm{BP} 29, \operatorname{Re}_{\omega}=71 \cdot 10^{4}, \beta=54,32^{\circ}$

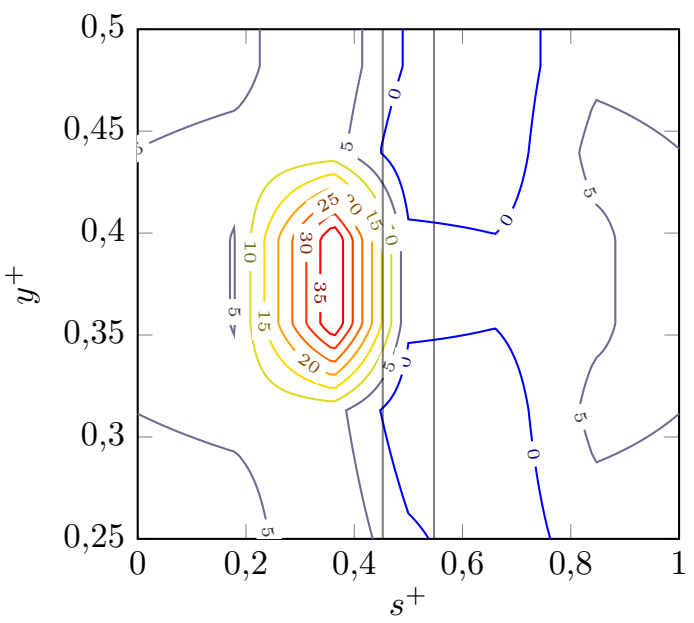

(e) $\mathrm{BP} 56, \operatorname{Re}_{\omega}=143 \cdot 10^{4}, \beta=72,21^{\circ}$

Abbildung 6.7: Einfluss der Drehzahl $\operatorname{Re}_{\omega}$ auf den flächengemittelten Wärmeübergang $\overline{\mathrm{Nu}}^{*}$ bei $\operatorname{Re}_{d}=1757$ und den ortsaufgelösten Wärmeübergang $\mathrm{Nu}$ 
werden auf der strömungszugewandten Flanke Wärmeübergangskoeffizienten von $\mathrm{Nu} \approx 20$ gemessen. In Richtung des Zahnkopfes ist eine leichte Erhöhung des Wärmeübergangs im Vergleich zum Zahnfuß festzustellen. Diese Tendenz unterscheidet sich qualitativ von der Wärmeübergangsverteilung für BP68 (siehe Abbildung 6.5c), an dem mit $\beta=57,95^{\circ}$ ein nahezu gleicher Aufprallwinkel vorliegt. Dort wird eine deutliche Konzentration des Wärmeübergangs in der Zahnmitte beobachtet.

Eine mögliche Ursache dieses Unterschieds liegt in der Strömungsform des Ölstrahls, der bei $\operatorname{Re}_{d}=1757$ eine glaskörperartige, gleichmäßige Struktur aufweist, die charakteristisch für laminare, anliegende Düsenströmungen ist. Bei $\mathrm{Re}_{d}=2639$ wird eine deutlich welligere, diffusere Struktur beobachtet, die auf einen abgelösten Strahl hinweist. In diesem Fall liegt durch die Einschnürung der Strömung eine höhere Strahlgeschwindigkeit vor, als sie die Berechnung anhand

$$
u_{\mathrm{s}}=\frac{\dot{V}}{A_{d}}
$$

erwarten lässt. Folglich liegt bei abgelöster Strömung tatsächlich ein höherer Aufprallwinkel als der berechnete vor.

Der Wärmeübergang auf der strömungsabgewandten Zahnflanke bei $\operatorname{Re}_{\omega}=71 \cdot 10^{4}(\mathrm{BP} 29$, s. Abb. 6.7c) ist im Vergleich zu $\operatorname{Re}_{\omega}=48 \cdot 10^{4}$ bereits deutlich reduziert. Bei weiterer Erhöhung auf $\operatorname{Re}_{\omega}=95 \cdot 10^{4}$ (BP54, s. Abb. 6.7d) kommt es zum beinahe vollständigen Erliegen des Wärmeübergangs auf der strömungsabgewandten Flanke. Im Bereich des Zahnkopfes auf der strömungsabgewandten Seite werden geringfügige, negative Wärmeübergangskoeffizienten gemessen.

Bei $\operatorname{Re}_{\omega}=95 \cdot 10^{4}$ und $\beta=62,89^{\circ}$ findet der Wärmeübergang nahezu ausschließlich am Zahnkopf der strömungszugewandten Flanke statt. Im Bereich des Zahnfußes sind nur noch geringe Nußeltzahlen von ca. 10 zu beobachten. Bei weiterer Erhöhung der Drehzahl auf $\operatorname{Re}_{\omega}=143 \cdot 10^{4}$ (BP56, s. Abb. 6.7e) ist ausschließlich am Zahnkopf signifikanter Wärmeübergang zu beobachten. Dies ist auf das sehr geringe Eindringen des Ölstrahls mit einem Aufprallwinkel von $\beta=72,21^{\circ}$ zurückzuführen, bei dem ausschließlich die strömungszugewandte Zahnflanke in der Nähe des Zahnkopfes vom Öl erreicht wird.

Die vorangegangene Betrachtung des Wärmeübergangs bei $\operatorname{Re}_{d}=2639$ und $\operatorname{Re}_{d}=1757$ soll im Folgenden in knapper Form bei erhöhtem Ölvolumenstrom mit $\mathrm{Re}_{d}=3954$ angestellt werden. In Abbildung 6.8a ist der Verlauf des mittleren Wärmeübergangs bei $\operatorname{Re}_{d}=3954$ in einem Drehzahlbereich von $\operatorname{Re}_{\omega}=48 \cdot 10^{4}$ bis $240 \cdot 10^{4}$ dargestellt. Erneut ist ein Bereich geringfügig ansteigender Werte von $\overline{\mathrm{Nu}^{*}}$ zu erkennen, der sich von $\operatorname{Re}_{\omega}=48 \cdot 10^{4}$ bis $145 \cdot 10^{4}$ bzw. $\beta=$ $50,14^{\circ}$ erstreckt. Bei höheren Drehzahlen fällt der mittlere Wärmeübergang ab. Insgesamt ist der mittlere Wärmeübergang auf einem höheren Niveau als für $\operatorname{Re}_{d}=2639$.

Bei $\operatorname{Re}_{\omega}=71 \cdot 10^{4}$ (BP38, s. Abb 6.7b) und $\beta=23,05^{\circ}$ ist der Wärmeübergang im Bereich des Zahnfußes konzentriert. Entlang der gesamten Linie $s^{+}=0,2$ nimmt der Wärmeübergang hohe Werte von bis zu Nu $\approx 40$ an. Auch auf der strömungsabgewandten Seite ist ein breiter Bereich hohen Wärmeübergangs zu beobachten. Dies entspricht den beobachteten Verteilungen für kleine Aufprallwinkel für $\operatorname{Re}_{d}=1757$ und $\operatorname{Re}_{d}=2639$. 
Mit Erhöhung der Drehzahl auf $\operatorname{Re}_{\omega}=143 \cdot 10^{4}$ (BP36, s. Abb. 6.8c) beträgt der kinematische Aufprallwinkel $\beta=50,14^{\circ}$. Wie auch bei den anderen betrachteten Ölvolumenströmen bzw. Werten für $\operatorname{Re}_{d}$ führen diese kinematischen Bedingungen zu einem Maximum des Wärmeübergangs im unteren Bereich der strömungszugewandten Flanke. Das Niveau des Wärmeübergangs ist im Vergleich zu Betriebspunkten bei niedrigeren Werten von $\operatorname{Re}_{d}$ und $\operatorname{Re}_{\omega}$, die einen ähnlichen Aufprallwinkel aufweisen, erhöht (siehe Abbildung 6.8c).

Bei $\operatorname{Re}_{\omega}=180 \cdot 10^{4}$ (BP18, s. Abb. 6.8d) ist bereits ein geringfügiger Rückgang von $\overline{\mathrm{Nu}} * \mathrm{im}$ Vergleich zu BP38 zu beobachten, der durch den Rückgang der örtlichen Wärmeübergangskoeffizienten auf der strömungsabgewandten Seite und auch in den äußeren Bereichen des Zahnfußes entsteht. Bei Erhöhung von $\operatorname{Re}_{\omega}$ (BP19, s. Abb. 6.8d) auf $240 \cdot 10^{4}$ und $\beta=66,48^{\circ}$ setzt sich diese Tendenz fort und der Wärmeübergang auf der strömungsabgewandten Seite trägt nur noch marginal zum gesamten Wärmeübergang bei. Bei dieser Drehzahl ist der maximale Wärmeübergang, wie bei anderen Betriebspunkten mit ähnlichem Aufprallwinkel, im Bereich des strömungszugewandten Zahnkopfes konzentriert. Es ist anzunehmen, dass diese Tendenz erneut durch das flachere Eindringen des Ölstrahls und die geringere Ölmenge, die bei großen Aufprallwinkeln die strömungsabgewandte Zahnflanke erreicht, hervorgerufen wird. 

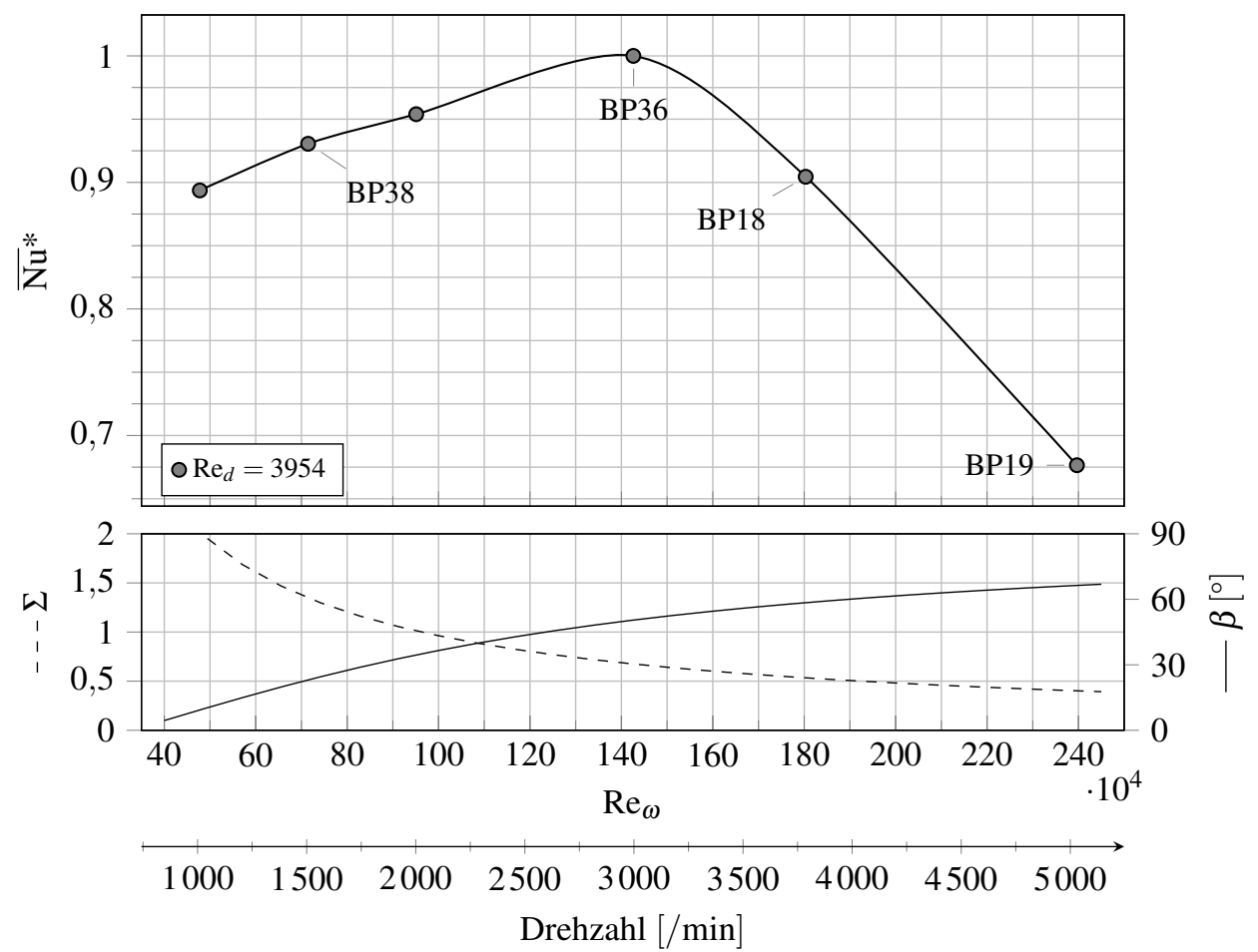

(a) Verlauf von $\overline{\mathrm{Nu}}^{*}, \Sigma$ und $\beta$ über $\operatorname{Re}_{\omega}$

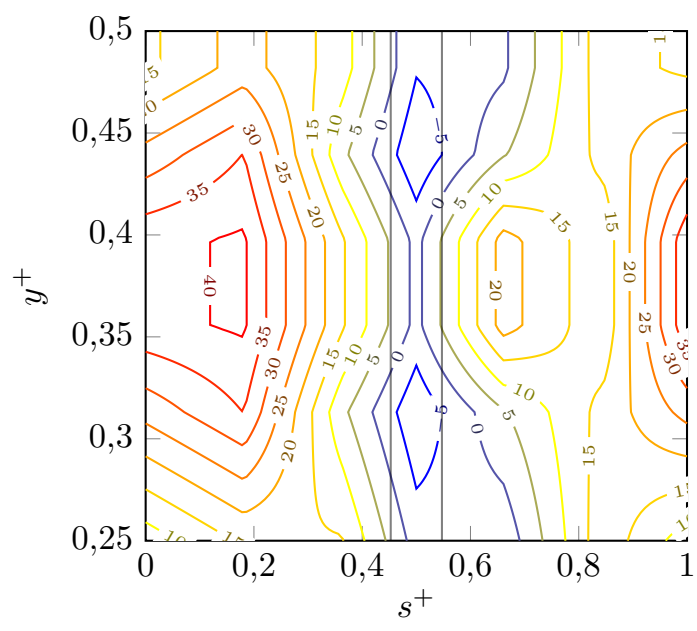

(b) $\mathrm{BP} 38, \operatorname{Re}_{\omega}=71 \cdot 10^{4}, \beta=23,05^{\circ}$

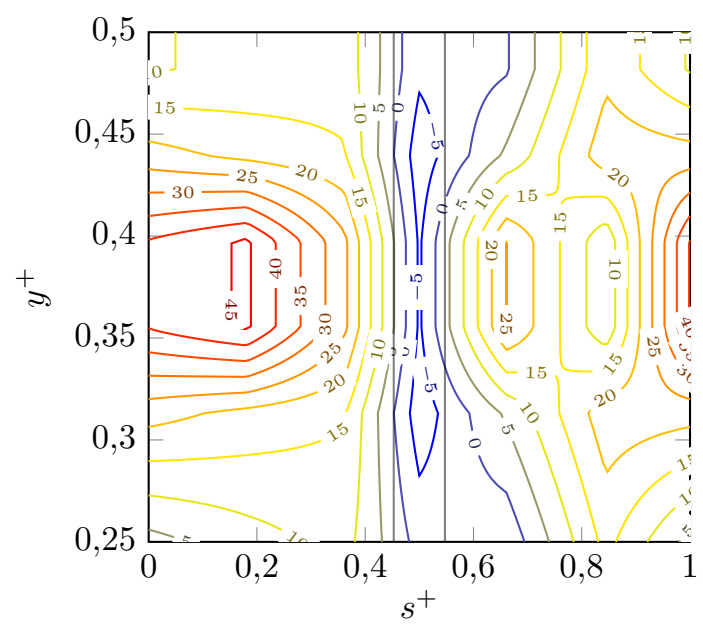

(d) $\mathrm{BP} 18, \operatorname{Re}_{\omega}=180 \cdot 10^{4}, \beta=58,36^{\circ}$

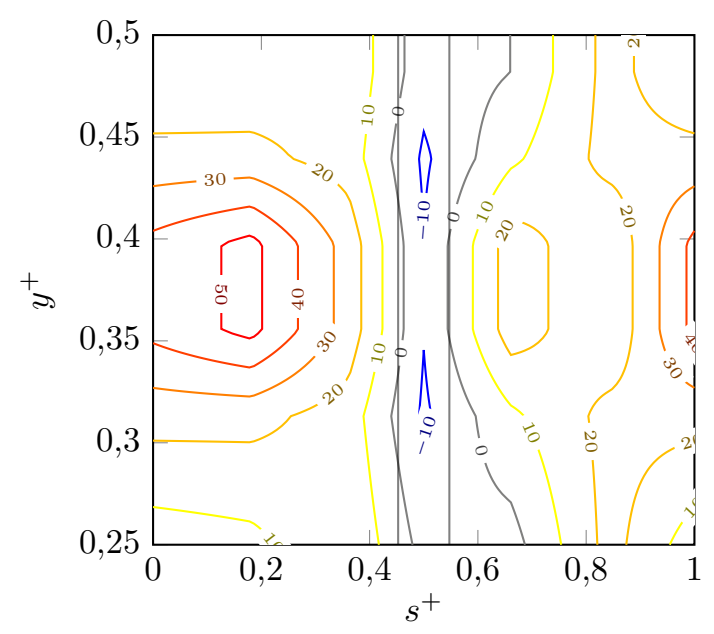

(c) $\mathrm{BP} 36, \operatorname{Re}_{\omega}=143 \cdot 10^{4}, \beta=50,14^{\circ}$

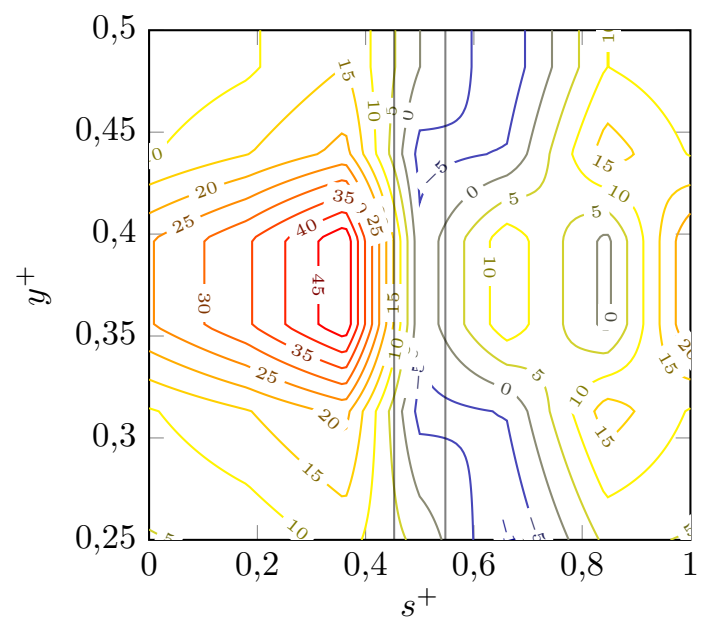

(e) $\mathrm{BP} 19, \operatorname{Re}_{\omega}=240 \cdot 10^{4}, \beta=66,48^{\circ}$

Abbildung 6.8: Einfluss der Drehzahl $\operatorname{Re}_{\omega}$ auf den flächengemittelten Wärmeübergang $\overline{\mathrm{Nu}}^{*}$ bei $\mathrm{Re}_{d}=3954$ und den ortsaufgelösten Wärmeübergang $\mathrm{Nu}$ 
Die im Vorangegangenen diskutierten Abhängigkeiten des Öl-Zahnrad-Wärmeübergangs zeigen eindeutige charakteristische Abhängigkeiten von der Strahlreynoldszahl $\operatorname{Re}_{d}$ und der Drehzahl $\operatorname{Re}_{\omega}$. In Abbildung 6.9 sind die Verläufe des mittleren Wärmeübergangs $\overline{\mathrm{Nu}} *$ für die diskutierten Ölvolumenströme mit $\operatorname{Re}_{d}=1757,2639$ und 3954 sowie Ergebnisse der dazwischen liegenden Werte von $\operatorname{Re}_{d}=2251$ und 3295 zusammengefasst dargestellt. Der Düsenwinkel beträgt stets $\alpha=20^{\circ}$. Es ist ersichtlich, dass der Verlauf von $\overline{\mathrm{Nu}}^{*}$ über $\operatorname{Re}_{\omega}$ bei $\operatorname{Re}_{d}=2251$ und 3295 qualitativ mit den bereits diskutierten Verläufen übereinstimmt. Die ortsaufgelösten Messungen des Wärmeübergangs, die hier nicht detailliert betrachtet werden sollen, zeigen die gleichen charakteristischen Muster und Vorgänge mit steigenden Drehzahlen, die zunächst zu einem Anstieg und anschließend zu einem Abfall des mittleren Wärmeübergangs führen. Steigende

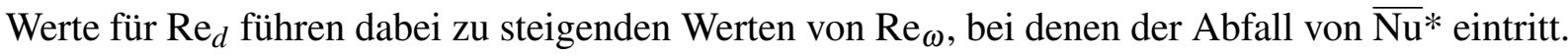

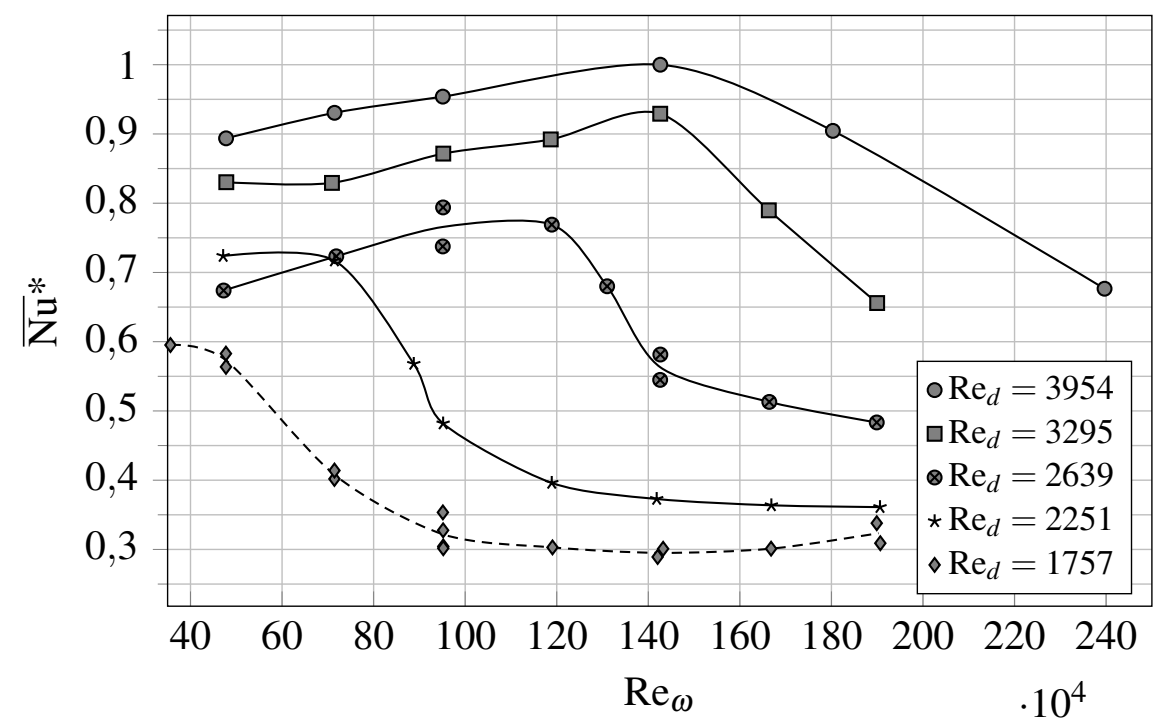

Abbildung 6.9: Verlauf von $\overline{\mathrm{Nu}^{*}}$ in Abhängigkeit von $\operatorname{Re}_{\omega}$ und $\operatorname{Re}_{d}$ und für $\alpha=20^{\circ}$

\section{Fazit}

In der vorangegangenen Analyse der Ergebnisse wurde erstmalig der Wärmeübergang an einem einspritzgekühlten Zahnrad isoliert von anderen Wärmeströmen und ortsaufgelöst beschrieben. Im betrachteten Betriebsbereich sind der eingespritzte Ölvolumenstrom, beschrieben durch $\operatorname{Re}_{d}$, und der mittlere Wärmeübergang $\overline{\mathrm{Nu}}^{*}$ stets positiv korreliert (siehe Abbildung 6.9). Zwischen der Drehzahl und dem Wärmeübergang besteht eine komplexe, nicht-monotone Abhängigkeit, die von einem deutlichen Rückgang von $\overline{\mathrm{Nu}}^{*}$ ab einer kritischen Drehzahl und der Verschiebung des Maximums des Wärmeübergangs vom Zahnfuß zum Zahnkopf gekennzeichnet ist. Durch die vereinfachte Betrachtung des Vorgangs als kinematisches Modell kann diese Verschiebung erklärt werden. Bei geringen Drehzahlen ist der Aufprallwinkel, unter dem der Ölstrahl in die Zahnlücke eindringt, gering. Dadurch erreicht das Öl den Zahnfuß und bewirkt hier ein Maximum des Wärmeübergangs. Mit steigenden Drehzahlen wird der Aufprallwinkel größer. Der Zahnfuß wird ab einem gewissen Aufprallwinkel nicht mehr erreicht und das Maximum des Wärmeübergangs verschiebt sich in Richtung des Zahnkopfes. Die Deutung durch den Aufprallwinkel wird durch die Gegenlichtaufnahmen bei $\operatorname{Re}_{d}=2639$ bestätigt. Der Rückgang von $\overline{\mathrm{Nu}} *$ kann 
durch die Gegenlichtaufnahmen ansatzweise erklärt werden. Bei Aufprallwinkeln von bis zu $\beta \approx 55^{\circ}$ prallt Öl an der strömungszugewandten Zahnflanke ab, trifft auf die gegenüberliegende, strömungsabgewandte Zahnflanke und führt dort zu signifikantem Wärmeübergang. Bei Aufprallwinkeln von über $\beta \approx 65^{\circ}$ erreicht das abprallende Öl die strömungsabgewandte Zahnflanke nicht mehr, wodurch dort der Wärmeübergang zum Erliegen kommt. Bei den drei diskutierten Ölvolumenströmen tritt der Rückgang bei Werten zwischen $\beta=50^{\circ}$ und $60^{\circ}$ auf. Durch die Änderung der Strömungsform in der Düse für verschiedene $\operatorname{Re}_{d}$ ist der kritische Winkel $\beta_{\text {cr }}$, bei dem der Rückgang von $\overline{\mathrm{Nu}}^{*}$ eintritt, in Fälle anliegender und abgelöster Strömung zu unterteilen. Diese Fallunterscheidung wird in Abschnitt 6.3 genauer erläutert.

\subsubsection{Einfluss des Düsenwinkels}

Untersuchungen von Keller et al. (2017) zeigen, dass der Düsenwinkel einen deutlichen Einfluss auf den Aufprallvorgang hat. Die Messungen des Wärmeübergangs weisen eine deutliche Abhängigkeit vom Düsenwinkel auf, wie im Folgenden gezeigt werden soll.

Zunächst soll der Verlauf des Wärmeübergangs für diskrete Düsenwinkel von $\alpha=20^{\circ}$, wie in Abschnitt 6.2.1, und $\alpha=45^{\circ}$ bei $\operatorname{Re}_{d}=2639$ und in einem Drehzahlbereich zwischen $\operatorname{Re}_{\omega}=48 \cdot 10^{4}$ und $190 \cdot 10^{4}$ diskutiert werden. Anschließend wird der Einfluss der Variation des Düsenwinkels bei ansonsten gleichbleibenden Bedingungen diskutiert.

In Abbildung 6.10 ist der Verlauf des mittleren Wärmeübergangs bei $\operatorname{Re}_{d}=2639$ dargestellt. Insgesamt liegt der mittlere Wärmeübergang für beide Düsenwinkel auf einem ähnlichen Niveau. Im Gegensatz zu den bisher betrachteten mittleren Wärmeübergangsverläufen bei $\alpha=20^{\circ}$ wird bei $\alpha=45^{\circ}$ eine monotone Abnahme mit zunehmender Drehzahl beobachtet. Mit steigender Drehzahl nimmt $\overline{\mathrm{Nu}} *$ stetig ab.

Für $\operatorname{Re}_{\omega} \geq 119 \cdot 10^{4}$ sind die Verläufe von $\overline{\mathrm{Nu}} *$ für beide Düsenwinkel etwa parallel, der mittlere Wärmeübergang ist für $\alpha=45^{\circ}$ stets geringer als für $\alpha=20^{\circ}$. In diesem Bereich liegen mit $\Sigma<0,6$ geringe Geschwindigkeitsverhältnisse vor. Dadurch ist der Einfluss des Düsenwinkels auf den Aufprallwinkel $\beta$ gering (siehe Abbildung 3.7 in Abschnitt 3.3).

Zur Erklärung des unterschiedlichen Wärmeübergangsniveaus kann die Darstellung des ortsaufgelösten Wärmeübergangs in Abbildung 6.11 herangezogen werden. Die Wärmeübergangsverteilungen für $\operatorname{Re}_{\omega}=119 \cdot 10^{4}$ bei $\alpha=20^{\circ}$ (BP68) und $\alpha=45^{\circ}$ (BP103) sind in den Abbildungen $6.11 \mathrm{a}$ und $6.11 \mathrm{~b}$ dargestellt. In beiden Fällen sind qualitativ ähnliche Verteilungen auf der strömungszugewandten Flanke zu erkennen. Das Maximum des Wärmeübergangs wird im Bereich der axialen Mitte der strömungszugewandten Zahnflanke bei etwa $s^{+}=0,2$ gemessen. Bei $\alpha=45^{\circ}$ ist der Bereich hohen Wärmeübergangs stärker in der Mitte konzentriert, während er bei $\alpha=20^{\circ}$ etwas gleichmäßiger verteilt ist. Auf der strömungsabgewandten Zahnflanke werden ebenfalls qualitativ ähnliche Wärmeübergangsverteilungen gemessen. Jedoch ist das Niveau bei $\alpha=45^{\circ}$ deutlich geringer als bei $\alpha=20^{\circ}$. Dieses geringere Niveau ist ursächlich für den geringeren mittleren Wärmeübergang.

Eine mögliche Ursache für diesen Niveauunterschied kann im unterschiedlichen Aufprall des Ölstrahls auf die Zahnflanke gesehen werden. Mit steigenden Düsenwinkeln sinkt die Neigung 


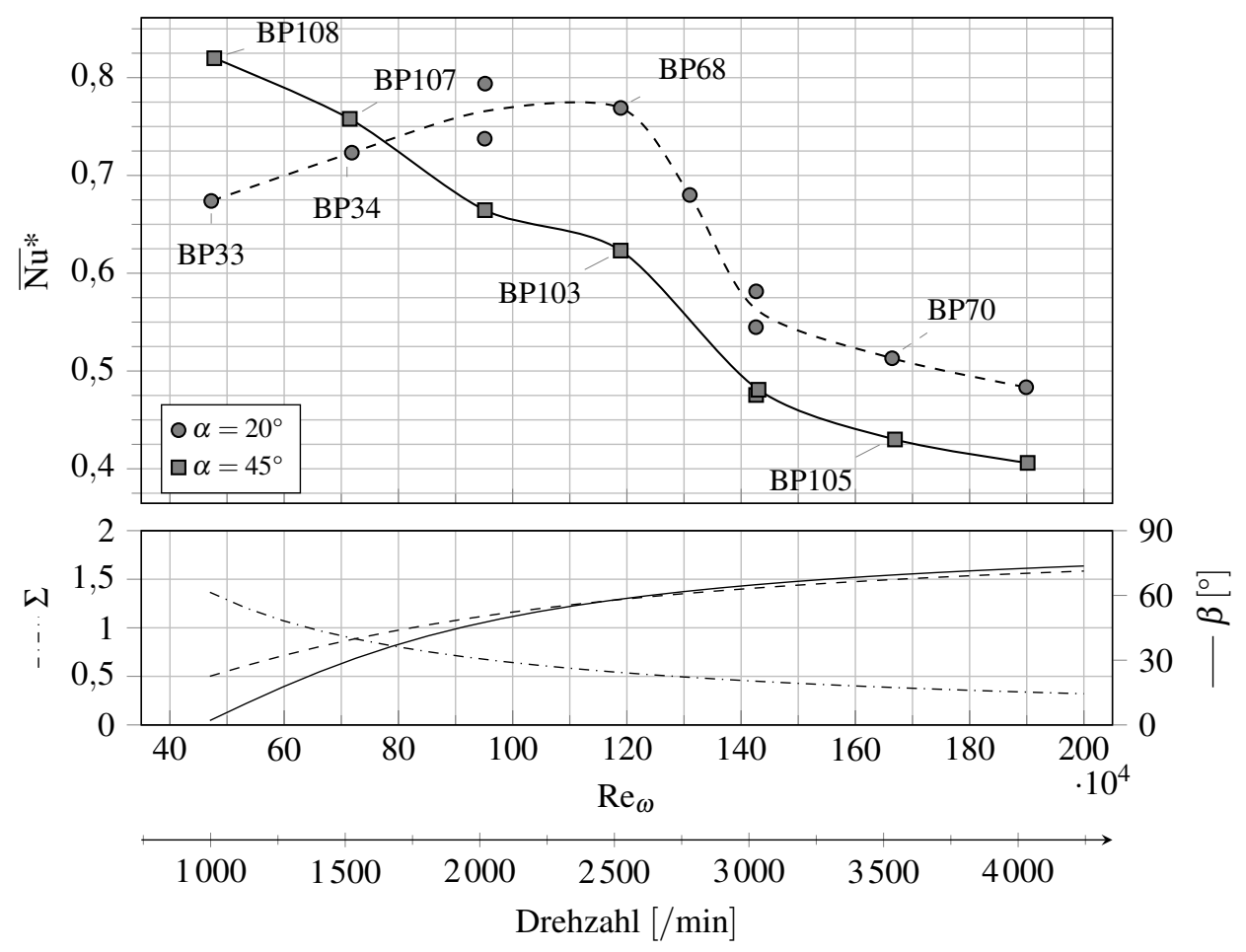

Abbildung 6.10: Einfluss des Düsenwinkels $\alpha$ auf den flächengemittelten Wärmeübergang $\overline{\mathrm{Nu}}$ * bei $\operatorname{Re}_{d}=2639$

des Ölstrahls, in Richtung der strömungsabgewandten Zahnflanke abzuprallen. Somit ist die Kühlwirkung an der strömungsabgewandten Zahnflanke bei zunehmendem $\alpha$ geringer. Dies führte bereits bei der Betrachtung des drehzahlabhängigen Wärmeübergangs bei hohen Drehzahlen zu einem Rückgang von $\overline{\mathrm{Nu}}^{*}$.

Bei einer auf $\operatorname{Re}_{\omega}=167 \cdot 10^{4}$ gesteigerten Drehzahl wird ein ähnlicher Effekt beobachtet. Die Wärmeübergangsverteilungen bei dieser Drehzahl sind in den Abbildungen 6.11c und 6.11d dargestellt. Bedingt durch den hohen Aufprallwinkel von etwa $\beta=68^{\circ}$ ist der Wärmeübergang wie in anderen betrachteten Fällen um den Zahnkopf konzentriert. Erneut ist die Verteilung von $\mathrm{Nu}$ bei $\alpha=20^{\circ}$ stark auf der strömungszugewandten Zahnflanke konzentriert und dort etwas gleichmäßiger verteilt. Der Bereich hohen Wärmeübergangs erstreckt sich bis zum Zahnfuß, während er bei $\alpha=45^{\circ}$ dort sehr gering ist. Bei $\alpha=20^{\circ}$ wird auf der strömungsabgewandten Zahnflanke noch ein geringfügiger Wärmeübergang gemessen, während er bei $\alpha=45^{\circ}$ dort nahezu zum Erliegen kommt. Wie bei $\operatorname{Re}_{d}=119 \cdot 10^{4}$ kann das geänderte Abprallverhalten des Öls für die verschiedenen Düsenwinkel als Ursache für diesen Unterschied gesehen werden. Das Öl erreicht bei $\alpha=45^{\circ}$ die strömungsabgewandte Zahnflanke nicht.

Im niedrigeren Bereich von $\operatorname{Re}_{\omega}$ zwischen $48 \cdot 10^{4}$ und $94 \cdot 10^{4}$ werden große qualitative Unterschiede des mittleren Wärmeübergangs für $\alpha=45^{\circ}$ und $\alpha=20^{\circ}$ gemessen, wie aus Abbildung 6.10 ersichtlich ist. Während bei $\alpha=20^{\circ}$ steigende Drehzahlen gleichbedeutend mit steigenden Werten von $\overline{\mathrm{Nu}}^{*}$ sind, sinkt der Wärmeübergang bei $\alpha=45^{\circ}$ in diesem Bereich kontinuierlich.

Die Verteilungen des Wärmeübergangs bei $\alpha=20^{\circ}$ und $\alpha=45^{\circ}$ unterscheiden sich sowohl 


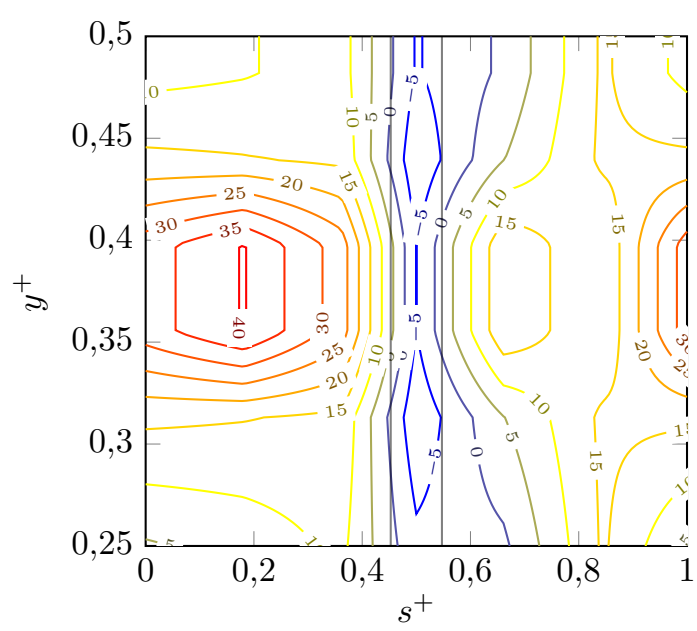

(a) $\mathrm{BP} 68, \operatorname{Re}_{\omega}=119 \cdot 10^{4}, \alpha=20^{\circ}, \beta=57,95^{\circ}$

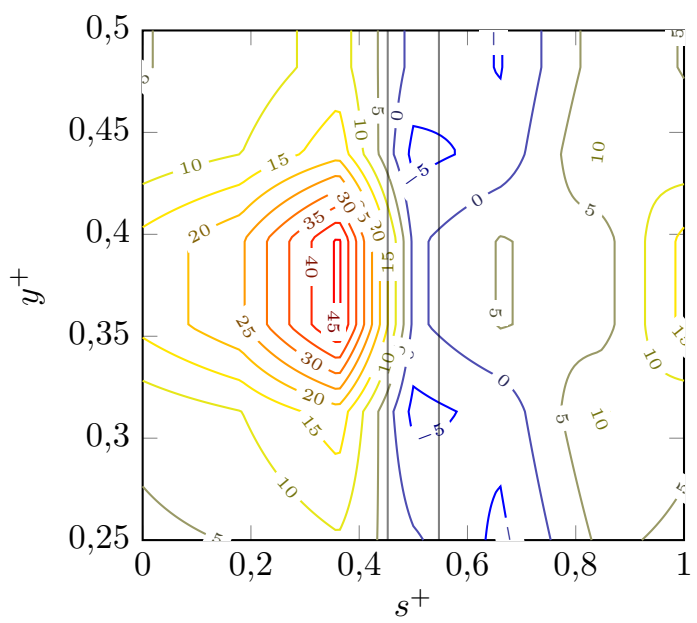

(c) $\mathrm{BP70}, \operatorname{Re}_{\omega}=167 \cdot 10^{4}, \alpha=20^{\circ}, \beta=67,22^{\circ}$

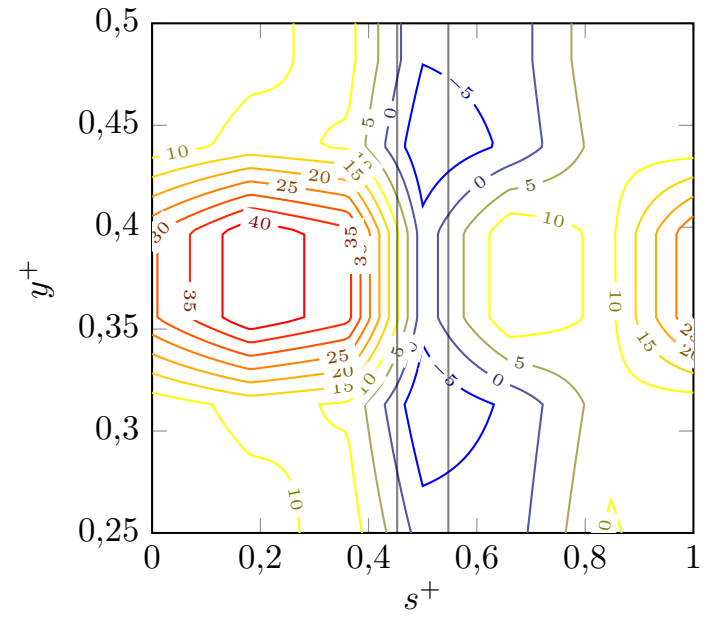

(b) $\mathrm{BP} 103, \operatorname{Re}_{\omega}=119 \cdot 10^{4}, \alpha=45^{\circ}, \beta=58,33^{\circ}$

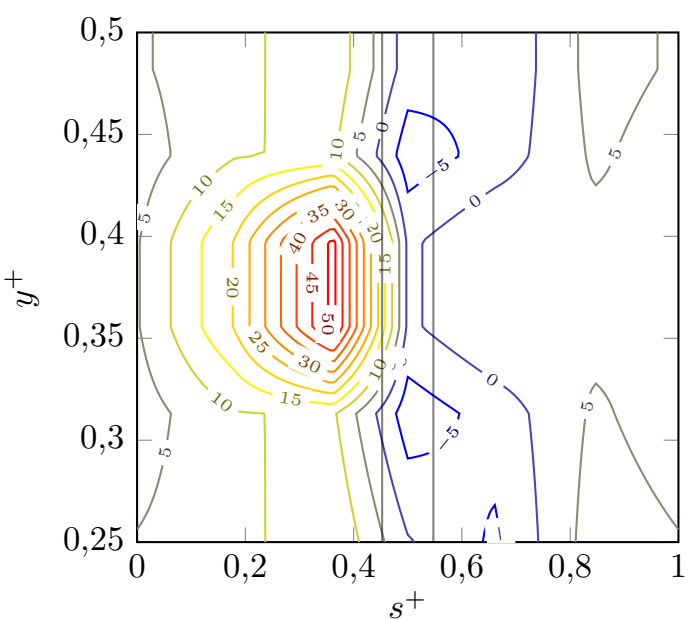

(d) $\mathrm{BP} 105, \operatorname{Re}_{\omega}=167 \cdot 10^{4}, \alpha=45^{\circ}, \beta=69,52^{\circ}$

Abbildung 6.11: Ortsaufgelöste Darstellung des Wärmeübergangs $\mathrm{Nu}$ bei $\operatorname{Re}_{d}=2639$ und hohen Drehzahlen

für $\operatorname{Re}_{\omega}=48 \cdot 10^{4}$ als auch für $\operatorname{Re}_{\omega}=71 \cdot 10^{4}$ stark, wie in Abbildung 6.12 deutlich wird. Bei $\alpha=45^{\circ}$ ist bei beiden Drehzahlen ein breiter Bereich in axialer Richtung in der Nähe des Zahnfußes auf der strömungszugewandten Flanke zu erkennen, der hohe Wärmeübergangskoeffizienten aufweist. Die Konzentration des Maximums in der Nähe des Zahnfußes entspricht den Erkenntnissen des vorangegangen Abschnitts für kleine Aufprallwinkel. Das Maximum in diesem Bereich ist jeweils geringfügig höher als das bei $\alpha=20^{\circ}$. Auch der Wärmeübergang auf der strömungsabgewandten Flanke ist erhöht, stellenweise um den Faktor zwei. Das geringfügig höhere Wärmeübergangsniveau wird durch den Aufprallwinkel erklärt, der für $\alpha=45^{\circ}$ bei gleicher Drehzahl $\operatorname{Re}_{\omega}$ stets kleiner ist als für $\alpha=20^{\circ}$. Im Fall von BP108 in Abbildung 6.12b mit $\operatorname{Re}_{\omega}=48 \cdot 10^{4}$ und $\alpha=45^{\circ}$ beträgt der Aufprallwinkel $\beta=3,06^{\circ}$, was einem nahezu senkrechten Eindringen des Ölstrahls in die Zahnlücke entspricht. Dabei wird, bei rein kinematischer Betrachtung des Vorgangs, auch die strömungsabgewandte Flanke direkt durch den Strahl getroffen. Dies kann als mögliche Ursache des erhöhten Wärmeübergangs an der strömungsabgewandten 
Flanke gesehen werden. Die große axiale Ausdehnung des Bereichs hohen Wärmeübergangs kann ein Hinweis auf ein axiales Ausbreiten des Öls am Zahnfuß gewertet werden, das durch das senkrechte Eindringen des Öls hervorgerufen wird. Bei $\alpha=20^{\circ}$ und ansonsten gleichbleibenden Bedingungen (BP, s. Abb. 6.12a) beträgt der Aufprallwinkel $\beta=22,49^{\circ}$ und ist somit deutlich flacher. In diesem Fall ist der Wärmeübergang an der strömungsabgewandten Flanke und seine axiale Ausbreitung am Zahnfuß geringer.

Bei einer auf $\operatorname{Re}_{\omega}=71 \cdot 10^{4}$ erhöhten Drehzahl wird für $\alpha=20^{\circ}$ (BP34) die in Abbildung 6.12c dargestellte Wärmeübergangsverteilung gemessen. Hier ist im Vergleich $\mathrm{zu} \operatorname{Re}_{\omega}=48 \cdot 10^{4}$ (BP33) eine Verschiebung des Wärmeübergangs in Richtung des Zahnkopfes erkennbar, was erneut durch den erhöhten Aufprallwinkel von $\beta=39,08^{\circ}$ erklärt wird. Bei $\operatorname{Re}_{\omega}=71 \cdot 10^{4}$ und $\alpha=45^{\circ}$ (BP107, s. Abb. 6.12d) beträgt der Aufprallwinkel $\beta=29,97^{\circ}$, was zu einem vorwiegend am Zahnfuß konzentrierten Wärmeübergang führt. Das hohe lokale Maximum von $\mathrm{Nu} \approx 25$ auf der strömungsabgewandten Flanke, das auch bei $\operatorname{Re}_{\omega}=48 \cdot 10^{4}$ gemessen wird, kann jedoch nicht durch den Aufprallwinkel erklärt werden. Möglicherweise ist die tatsächliche Strahlgeschwindigkeit $u_{\mathrm{s}}$ aufgrund eines abgelösten Ölstrahls höher als die berechnete, wodurch die strömungsabgewandte Zahnflanke auch bei $\operatorname{Re}_{\omega}=71 \cdot 10^{4}$ und $\alpha=45^{\circ}$ vom Öl erreicht wird.

Die Verteilungen des Wärmeübergangs bei $\alpha=45^{\circ}$ und $\operatorname{Re}_{\omega}=48 \cdot 10^{4}$ und $71 \cdot 10^{4}$ in Abbildung $6.12 \mathrm{~b}$ und $6.12 \mathrm{~d}$ weisen auf der strömungsabgewandten Flanke ein lokales Minimum bei $y^{+}=0,375$ und etwa $s^{+}=0,83$ auf. Auf Grundlage der verfügbaren Hochgeschwindigkeitsaufnahmen und aus Simulationen geht nicht hervor, wodurch dieser Effekt entsteht. Die Linie $s^{+}=0,83$ liegt jedoch im konkaven Bereich des Zahnfußes und wird möglicherweise nicht von an der strömungsabgewandten Zahnflanke abprallendem Öl getroffen. Für eine Überprüfung dieser Beobachtung und dem unerwartet hohen Wärmeübergang auf der strömungsabgewandten Zahnflanke bei $\operatorname{Re}_{\omega}=71 \cdot 10^{4}$ und $\alpha=45^{\circ}$ sind zusätzliche Informationen aus Experimenten oder numerischen Simulationen notwendig. 


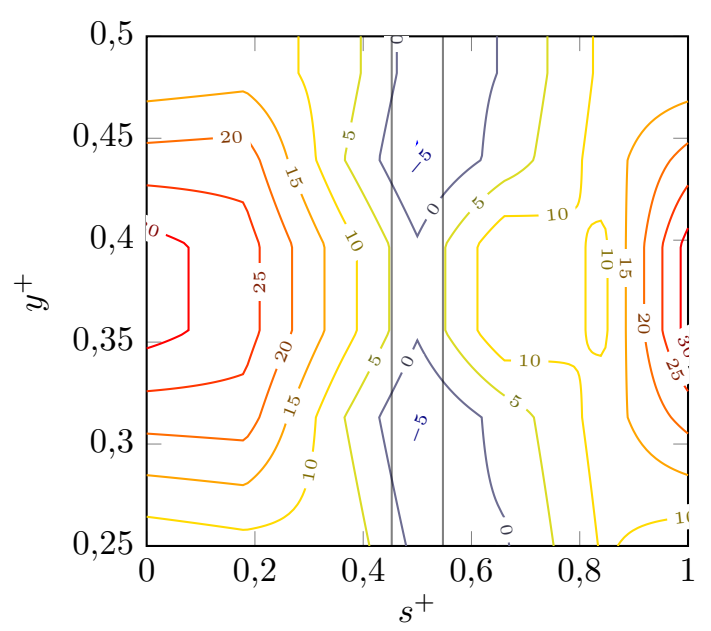

(a) $\mathrm{BP} 33, \operatorname{Re}_{\omega}=48 \cdot 10^{4}, \alpha=20^{\circ}, \beta=22,49^{\circ}$

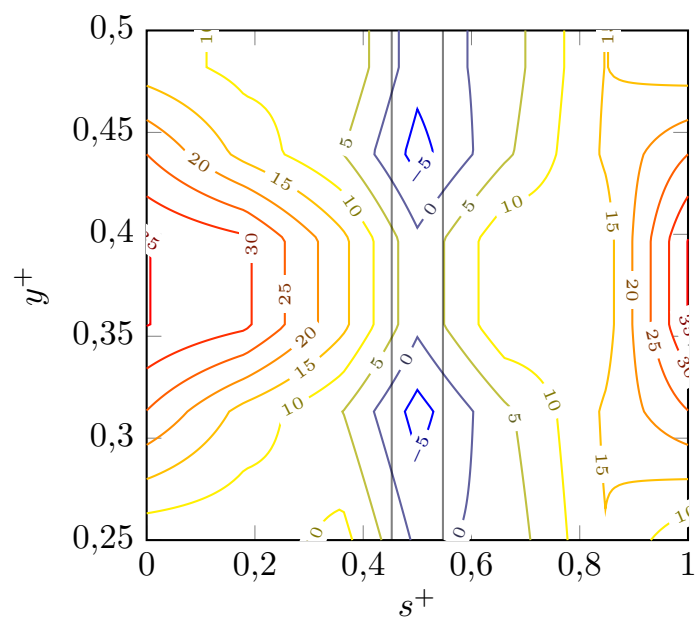

(c) $\mathrm{BP} 34, \operatorname{Re}_{\omega}=71 \cdot 10^{4}, \alpha=20^{\circ}, \beta=39,08^{\circ}$

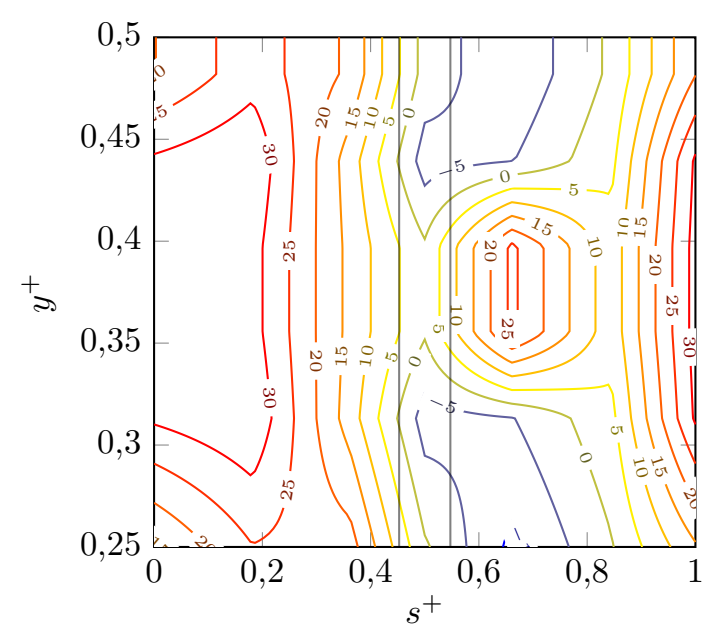

(b) $\mathrm{BP} 108, \operatorname{Re}_{\omega}=48 \cdot 10^{4}, \alpha=45^{\circ}, \beta=3,06^{\circ}$

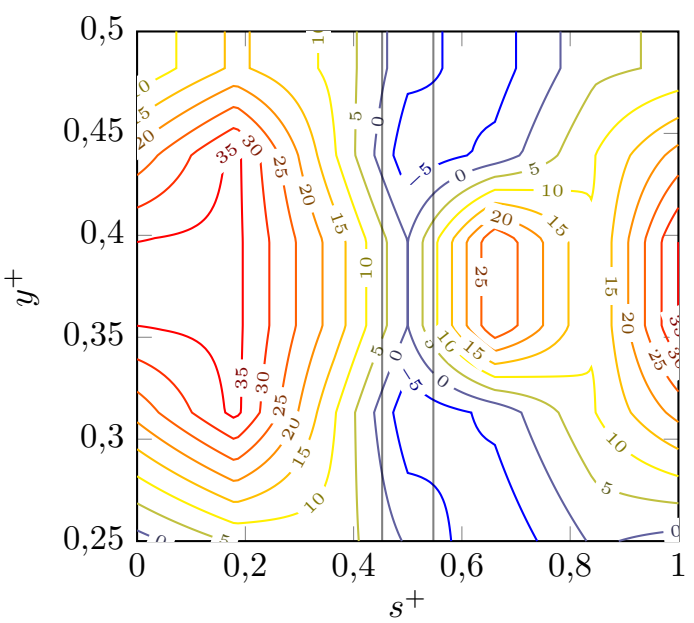

(d) $\mathrm{BP} 107, \operatorname{Re}_{\omega}=71 \cdot 10^{4}, \alpha=45^{\circ}, \beta=29,97^{\circ}$

Abbildung 6.12: Ortsaufgelöste Darstellung des Wärmeübergangs $\mathrm{Nu}$ bei $\mathrm{Re}_{d}=2639$ und niedrigen Drehzahlen

Um den Einfluss des Düsenwinkels eingehender zu betrachten, ist der Verlauf von $\overline{\mathrm{Nu}}$ bei $\operatorname{Re}_{d}=2639$ und $\operatorname{Re}_{\omega}=143 \cdot 10^{4}$ im Bereich von $\alpha$ von $-30^{\circ}$ bis $45^{\circ}$ in Abbildung 6.13 dargestellt. $\mathrm{Da} \operatorname{Re}_{d}, \operatorname{Re}_{\omega}$ und somit $\Sigma$ für die betrachteten Betriebspunkte konstant sind, ist der Aufprallwinkel $\beta$ eine Funktion von $\alpha$ mit einem Minimum bei $\alpha \approx 25^{\circ}$. Im Bereich zwischen $\alpha=-30^{\circ}$ und $20^{\circ}$ ist der mittlere Wärmeübergang nahezu konstant. Bei einer Vergrößerung des Düsenwinkels darüber hinaus in Richtung positiver Werte ist ein Absinken des mittleren Wärmeübergangs zu beobachten.

Durch die Analyse des ortsaufgelösten Wärmeübergangs kann auf die Ursachen des Verlaufs von $\overline{\mathrm{Nu}}^{*}$ in Abhängigkeit von $\alpha$ geschlossen werden. In Abbildung 6.14 sind die Wärmeübergangsverteilungen der Betriebspunkte 47, 44, 43 und 104 dargestellt. Bei einem Düsenwinkel von $\alpha=-30^{\circ}$ und $\beta=72,51^{\circ}$ für BP47 in Abbildung 6.14a wird eine Wärmeübergangsverteilung gemessen, deren Maximum im Bereich des Zahnkopfes liegt. Dies entspricht der Erwartung aus den vorangegangenen Betrachtungen für ähnlich flache Aufprallwinkel. Bei einer Erhöhung des 


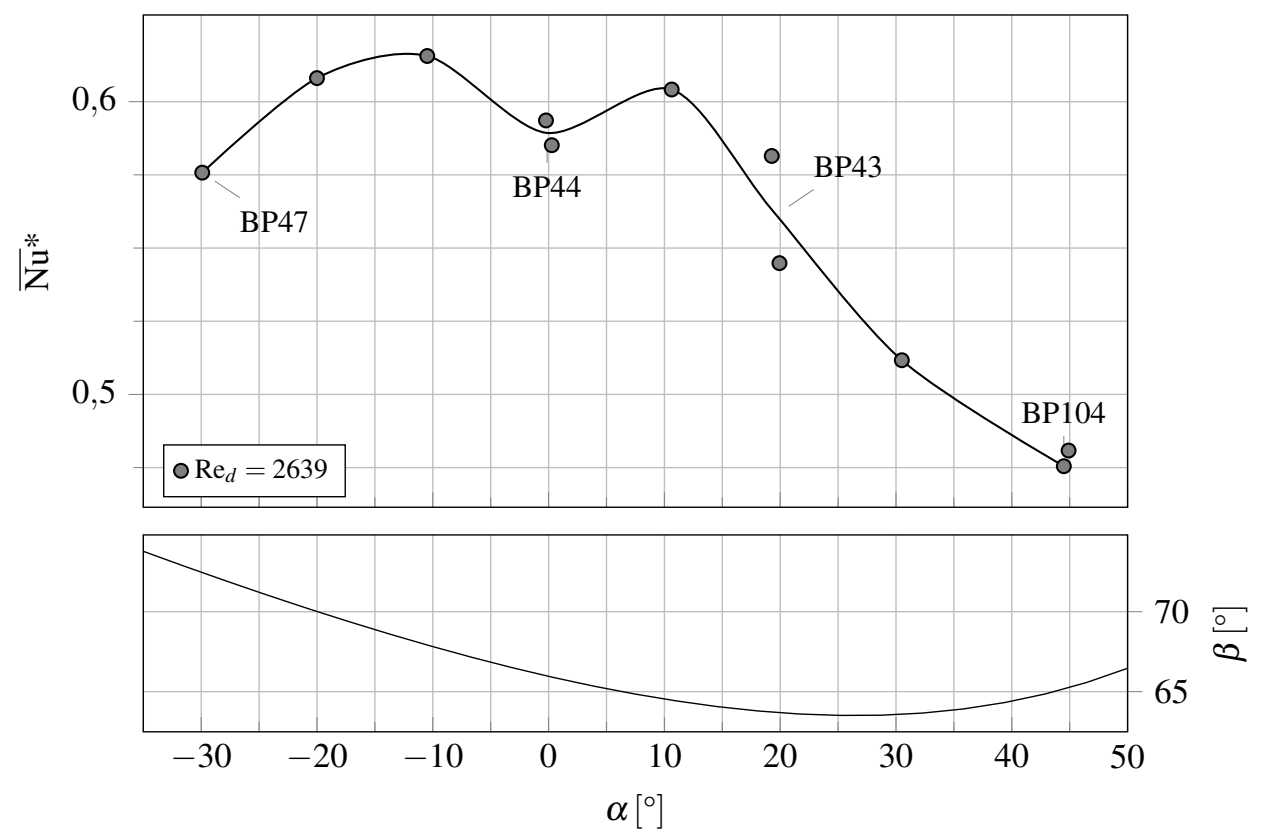

Abbildung 6.13: Einfluss des Düsenwinkels $\alpha$ auf den flächengemittelten Wärmeübergang $\overline{\mathrm{Nu}}$ * bei $\operatorname{Re}_{d}=2639$ und $\operatorname{Re}_{\omega}=143 \cdot 10^{4}$

Düsenwinkels auf $\alpha=0^{\circ}$ (BP44) ist nur eine geringfügige Erhöhung des mittleren Wärmeübergangs zu beobachten. Die Verteilung des Wärmeübergangs zeigt jedoch deutliche Unterschiede (siehe Abbildung 6.14b). Der Bereich hohen Wärmeübergangs erstreckt sich über einen größeren Bereich der strömungszugewandten Zahnflanke, wodurch der mittlere Wärmeübergang erhöht wird. Diese Verschiebung ist auf den steileren Aufprallwinkel und das damit verbundene tiefere Eindringen des Öls zurückzuführen. Auf der strömungsabgewandten Zahnflanke ist jedoch ein geringfügiger Rückgang des Wärmeübergangs zu verzeichnen, was einen gegenläufigen Effekt darstellt. Dieser Rückgang kann als Hinweis auf geändertes Abprallen des Ölstrahls und reduziertes Auftreffen auf der strömungsabgewandten Flanke gewertet werden. Die beiden gegenläufigen Effekte gleichen sich im Bereich von $\alpha=-30^{\circ}$ bis $10^{\circ}$ gegenseitig aus, wodurch $\overline{\mathrm{Nu}}^{*}$ etwa konstant bleibt.

Der Effekt der Umverteilung des Wärmeübergangs setzt sich für größere Düsenwinkel fort. Aus der in Abbildung 6.14c dargestellten Wärmeübergangsverteilung für $\alpha=20^{\circ}$ (BP43) ist eine wesentlich gleichmäßigere Verteilung auf der strömungszugewandten Zahnflanke erkennbar. Der Rückgang des Wärmeübergangs an der strömungsabgewandten Zahnflanke überwiegt, es kommt

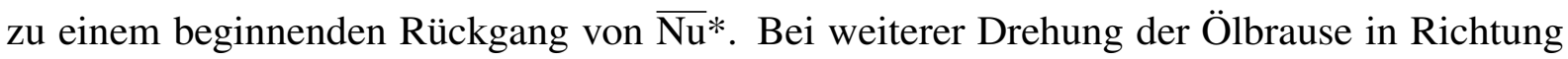
der Zahnradrotation zu $\alpha=45^{\circ}$ (BP104) wird eine Wärmeübergangsverteilung gemessen, die auf der gesamten Höhe der strömungszugewandten Zahnflanke hohe Werte aufweist und etwa die Form eines Rechtecks annimmt. Der Wärmeübergang auf der strömungsabgewandten Seite ist im Vergleich zum Wärmeübergang bei kleineren Düsenwinkeln deutlich reduziert. Der Rückgang des dortigen Wärmeübergangs verursacht maßgeblich die Reduktion des mittleren Wärmeübergangs $\overline{\mathrm{Nu}}^{*}$. 


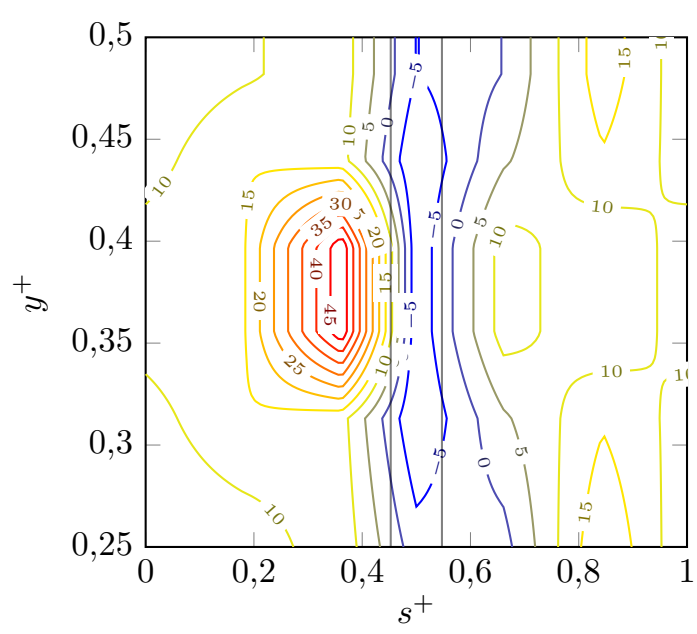

(a) BP47, $\alpha=-30^{\circ}, \beta=72,52^{\circ}$

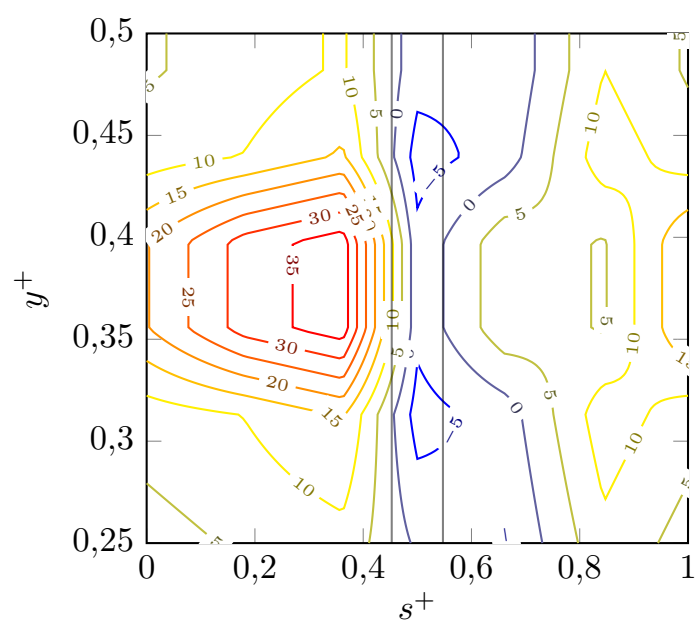

(c) $\mathrm{BP} 43, \alpha=20^{\circ}, \beta=63,80^{\circ}$

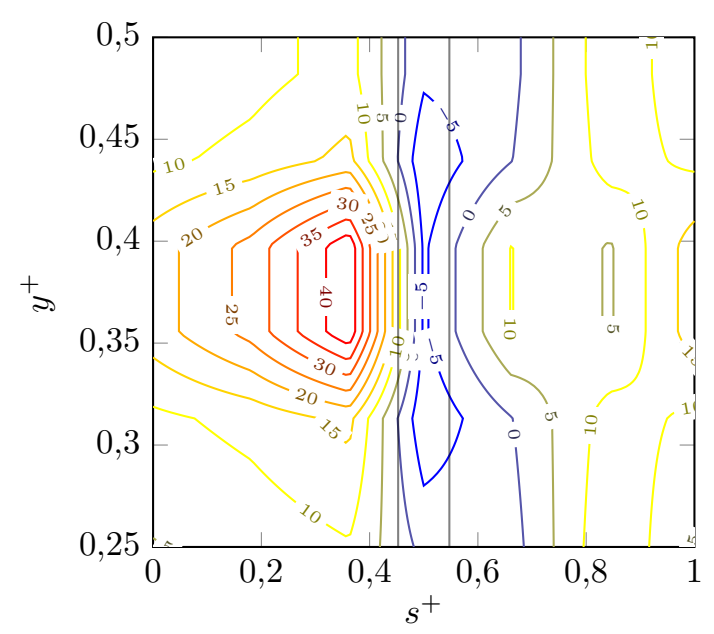

(b) BP44, $\alpha=0^{\circ}, \beta=66,03^{\circ}$

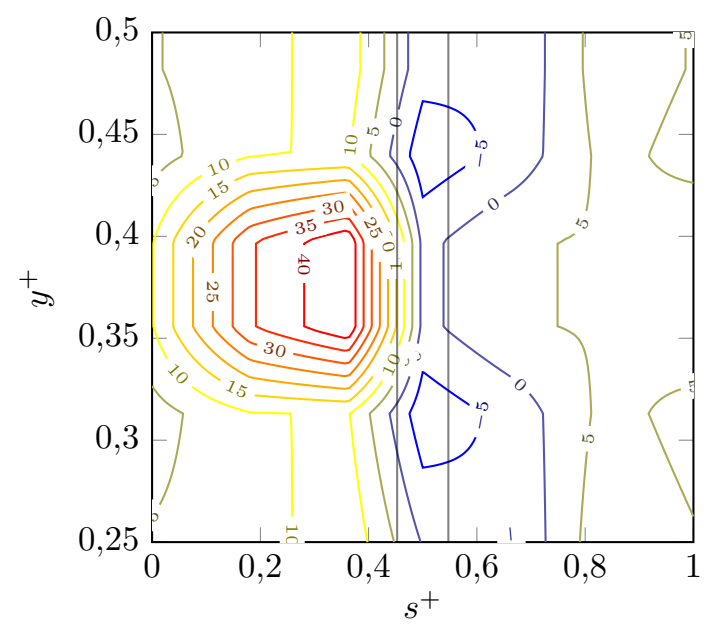

(d) $\mathrm{BP} 104, \alpha=45^{\circ}, \beta=65,07^{\circ}$

Abbildung 6.14: Einfluss des Düsenwinkels $\alpha$ auf die Wärmeübergangsverteilung Nu bei $\operatorname{Re}_{d}=2639$ und $\operatorname{Re}_{\omega}=143 \cdot 10^{4}$

\section{Fazit}

Die vorangegangene Betrachtung liefert die Erkenntnis, dass der Düsenwinkel sowohl den mittleren Wärmeübergang als auch die Verteilung des Wärmeübergangs beeinflusst. Größere Düsenwinkel führen dabei zu einer gleichmäßigeren Verteilung des Wärmeübergangs auf der strömungszugewandten Zahnflanke und tendenziell höheren Wärmeübergängen in diesem Bereich. Auf der gegenüberliegenden, strömungsabgewandten Zahnflanke werden abnehmende Wärmeübergänge beobachtet. Im betrachteten Fall überwiegt dieser Rückgang ab einem Düsenwinkel von $\alpha=20^{\circ}$, was zu geringeren Werten von $\overline{\mathrm{Nu}}^{*}$ führt. Die Vor- und Nachteile eines auf die strömungszugewandte Zahnflanke konzentrierten Wärmeübergangs sind vom Anwendungsfall abhängig und können im Rahmen dieser Arbeit nicht bewertet werden. Die diskutierten Messungen zeigen jedoch, dass die Wärmeübergangsverteilung durch den Düsenwinkel gezielt beeinflusst werden kann. Insgesamt ist die Abhängigkeit des mittleren Wärmeübergangs vom 
Düsenwinkel wesentlich geringer als seine Abhängigkeit von der Drehzahl und dem Ölvolumenstrom.

\subsubsection{Einfluss der Temperatur}

Um den Einfluss der Öltemperatur auf den Wärmeübergang zu quantifizieren, wird im Folgenden der Verlauf von $\overline{\mathrm{Nu}}^{*}$ in Abhängigkeit der Drehzahl bei $\alpha=20^{\circ}$ und einem Ölvolumenstrom von $\dot{V}=4,81 / \mathrm{min}$ bei $\vartheta_{\mathrm{s}}=60^{\circ} \mathrm{C}$ mit dem Verlauf von $\overline{\mathrm{Nu}}^{*}$ bei $\vartheta_{\mathrm{s}}=80^{\circ} \mathrm{C}$ und ansonsten identischen Parametern verglichen. Die Messungen bei $\vartheta_{\mathrm{s}}=80^{\circ} \mathrm{C}$ wurden im vorangegangenen Abschnitt diskutiert. In diesem Vergleich sind $\Sigma$ und $\beta$ für beide Temperaturen gleich, da der Ölvolumenstrom in beiden Fällen gleich ist. Die Strahlreynoldszahlen sind aufgrund der temperaturabhängigen Viskosität des verwendeten Öls verschieden und betragen $\operatorname{Re}_{d}=1619$

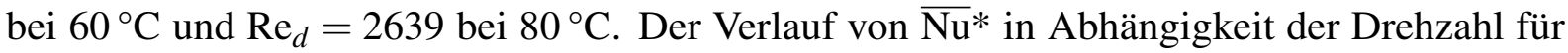
$\vartheta_{\mathrm{s}}=60^{\circ} \mathrm{C}$ ist in Abbildung 6.15a dargestellt.

Über den betrachteten Drehzahlbereich ist ein kontinuierlicher Rückgang des Wärmeübergangs mit Erhöhung der Drehzahl bis auf $\operatorname{Re}_{\omega}=84 \cdot 10^{4}$ zu beobachten. Während bei $\operatorname{Re}_{\omega}=28 \cdot 10^{4}$ mittlere Nußeltzahlen von etwa 0,8 gemessen werden, sinkt dieser Wert auf ein Minimum von etwa 0,46 bei $\operatorname{Re}_{\omega}=84 \cdot 10^{4}$. Aus den Darstellungen des ortsaufgelösten Wärmeübergangs in Abbildungen 6.15b bis 6.15e wird deutlich, dass wie in allen bisher beobachteten Fällen das Maximum des Wärmeübergangs mit steigender Drehzahl vom Zahnfuß zum Zahnkopf verschoben wird. Der Wärmeübergang an der strömungsabgewandten Zahnflanke nimmt, wie auch bei den Messungen bei $\vartheta_{\mathrm{s}}=80^{\circ} \mathrm{C}$, kontinuierlich ab und führt zu einer Abnahme des mittleren Wärmeübergangs bei erhöhten Drehzahlen.

Die Verschiebung des Wärmeübergangsmaximums auf der strömungszugewandten Zahnflanke zum Zahnkopf beginnt im Vergleich zu den Messungen bei $\vartheta_{\mathrm{s}}=80^{\circ} \mathrm{C}$ und $\operatorname{Re}_{d}=2639$ (Abb. 6.5) bei wesentlich niedrigeren Drehzahlen bzw. Aufprallwinkeln. Bereits bei $\operatorname{Re}_{\omega}=70 \cdot 10^{4}$ und $\beta=58,73^{\circ}$ (BP123 Abbildung 6.15d) wird im Bereich des Zahnkopfes ein erhöhter Wärmeübergang gemessen. Diese Verschiebung wurde bei $\vartheta_{\mathrm{s}}=80^{\circ} \mathrm{C}$ erst bei $\beta \approx 67^{\circ}$ beobachtet. Eine mögliche Ursache ist in der geringeren Strahlreynoldszahl zu sehen, bei der noch keine Ablösung des Strahls zu erwarten ist. Hierdurch sind die Strahlgeschwindigkeit $u_{\mathrm{s}}$ und der tatsächliche Aufprallwinkel stets geringer als für abgelöste Strahlen, was zu einer geringeren Eindringtiefe führt.

Der mittlere Wärmeübergang ist für BP123 im Vergleich zu niedrigeren Drehzahlen deutlich reduziert. Der Wärmeübergang an der strömungsabgewandten Zahnflanke ist bereits erheblich zurückgegangen (siehe Abb. 6.15d). In Betriebspunkten bei $80^{\circ} \mathrm{C}$ mit ähnlichen Aufprallwinkeln, bei denen beginnender kopflastiger Wärmeübergang gemessen wird, ist der Wärmeübergang auf der strömungsabgewandten Flanke noch wesentlich stärker ausgeprägt (vgl. BP29, Abb. 6.7c und BP43, Abb. 6.5d). Dies kann mit der durch geringere Temperaturen erhöhten Viskosität des Öls zusammenhängen, die das Abprallen des Öls durch Dissipationsvorgänge hemmt. Für eine Überprüfung dieses Effektes sind jedoch numerische Strömungsuntersuchungen erforderlich. 

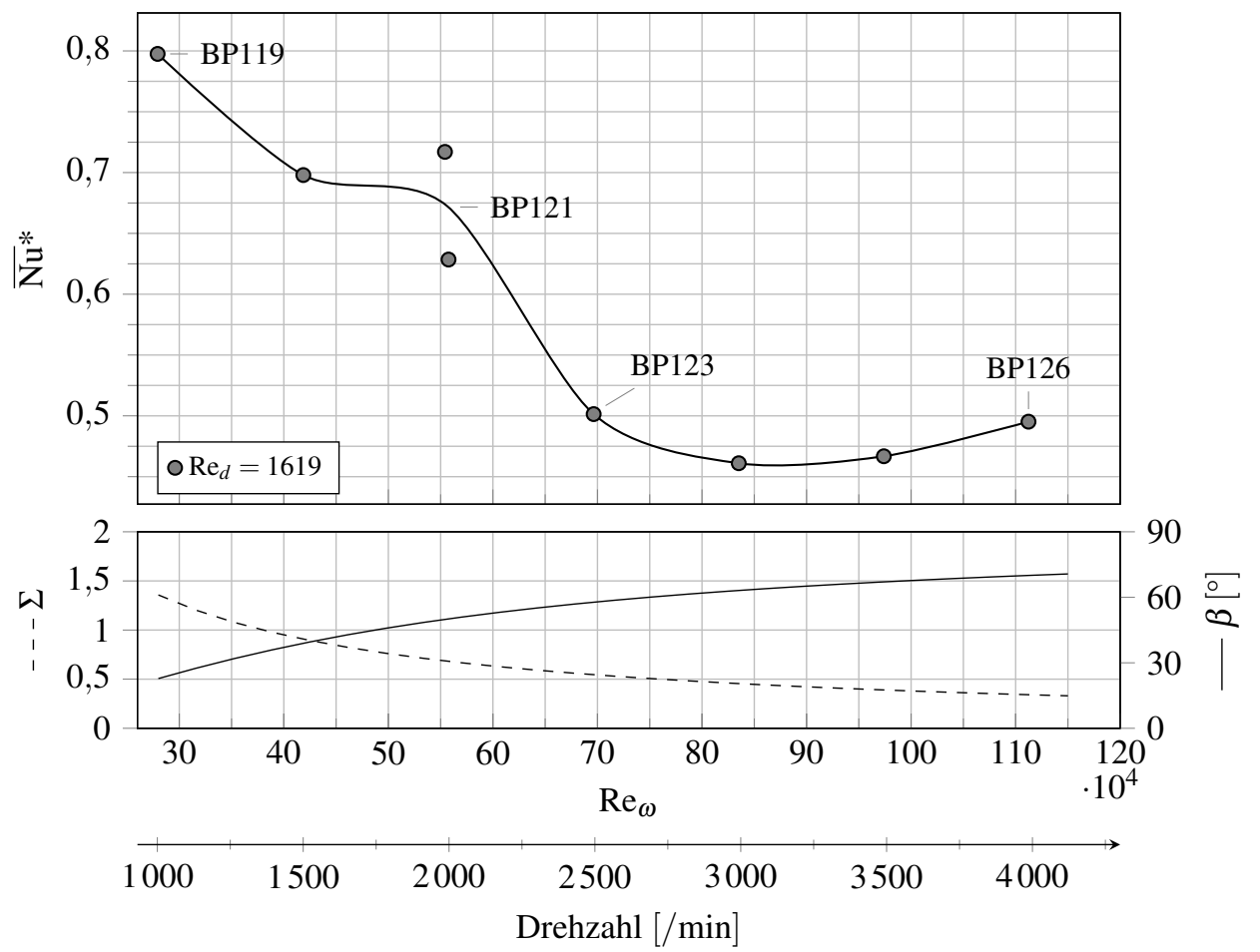

(a) Verlauf von $\overline{\mathrm{Nu}}^{*}, \Sigma$ und $\beta$ über $\operatorname{Re}_{\omega}$

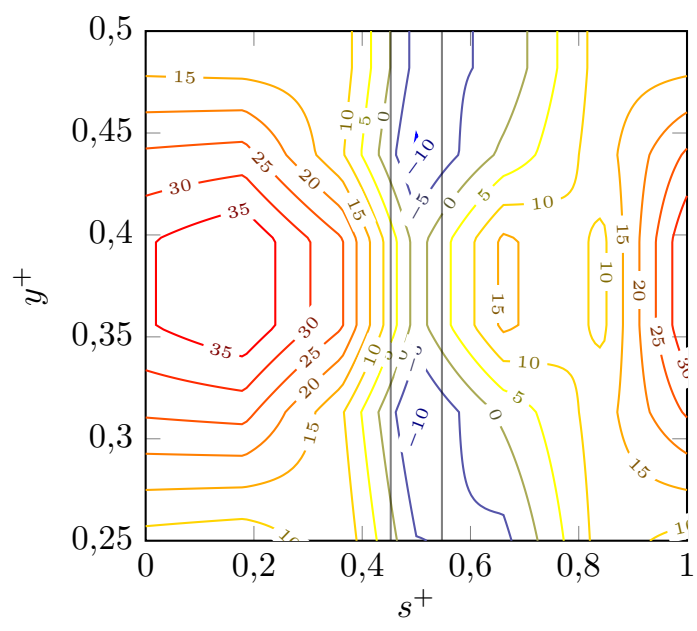

(b) $\mathrm{BP} 119, \operatorname{Re}_{\omega}=28 \cdot 10^{4}, \beta=24,15^{\circ}$

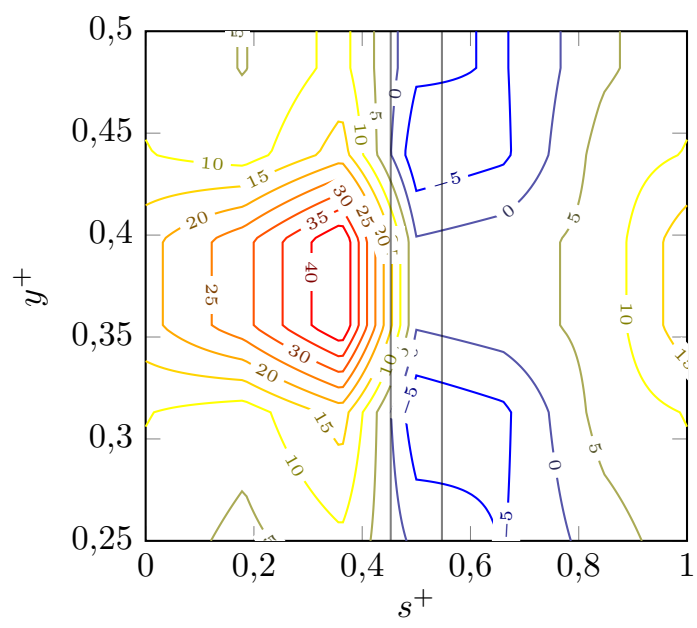

(d) $\mathrm{BP} 123, \operatorname{Re}_{\omega}=70 \cdot 10^{4}, \beta=58,73^{\circ}$

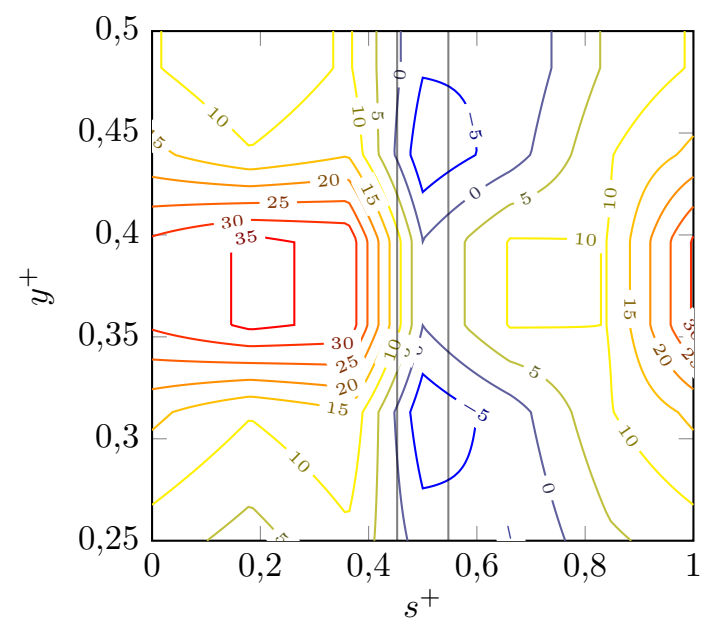

(c) $\mathrm{BP} 121, \operatorname{Re}_{\omega}=56 \cdot 10^{4}, \beta=51,25^{\circ}$

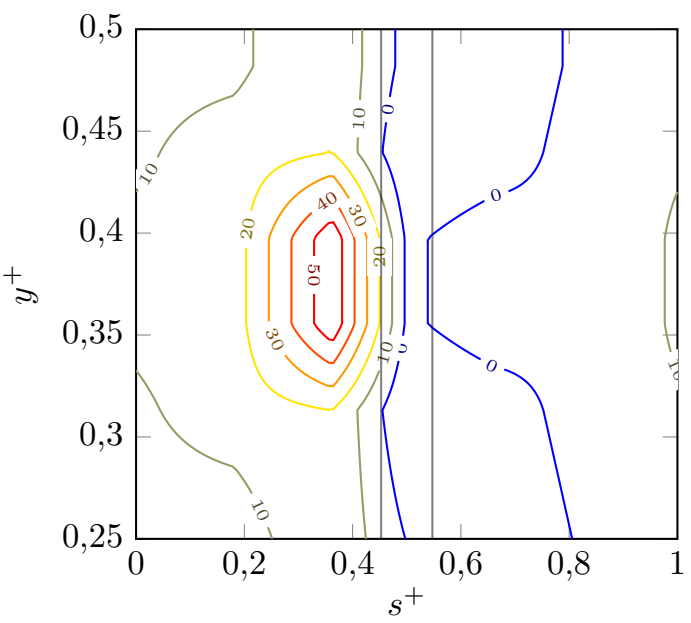

(e) $\mathrm{BP} 126, \operatorname{Re}_{\omega}=112 \cdot 10^{4}, \beta=70,64^{\circ}$

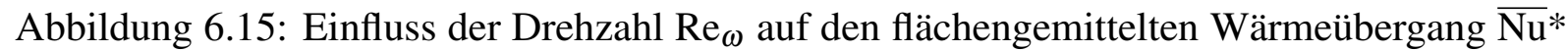
bei $\operatorname{Re}_{d}=1619$ und den ortsaufgelösten Wärmeübergang $\mathrm{Nu}$ bei reduzierter Öltemperatur $\vartheta_{\mathrm{s}}=60^{\circ} \mathrm{C}$ 


\subsection{Ableitung einer Korrelation aus den gewonnenen Daten}

Aus der Diskussion der Ergebnisse in Abschnitt 6.2 wird deutlich, dass alle der untersuchten Parameter

- Drehzahl $n$

- Ölvolumenstrom $\dot{V}$

- Düsenwinkel $\alpha$

- Öltemperatur $\vartheta_{\mathrm{s}}$

den Wärmeübergang am Zahnrad beeinflussen. Jedoch zeigen die Verläufe des mittleren Wärmeübergangs $\overline{\mathrm{Nu}}$ ein ähnliches Verhalten, wenn sie in Abhängigkeit des Aufprallwinkels $\beta$ betrachtet werden, der aus $\dot{V}, n$ und $\alpha$ berechnet wird. Für die Entwicklung von Höchstleistungsgetrieben ist es von Interesse, die im Rahmen dieser Arbeit gewonnenen Daten in vereinfachter Form abzubilden. Dies legt es nahe, die gemessenen mittleren Wärmeübergangskoeffizienten durch eine Korrelation auszudrücken, die die beobachteten charakteristischen Einflüsse berücksichtigt. Eine solche Korrelation soll im Folgenden beschrieben werden.

Die Messung des ortsaufgelösten Wärmeübergangs ergibt qualitativ ähnliche Verteilungen bei gleichen Werten für $\beta$. Außerdem wird durch die Analyse des Stands der Wissenschaft zur Prallkühlung durch Flüssigkeitsstrahlen deutlich, dass der Wärmeübergang an unbewegten ebenen Oberflächen stets eine Proportionalität zu Re ${ }^{0,5}$ und somit zur Wurzel des Impulses des Strahls besitzt. Wird im Fall der Zahnradkühlung die Relativgeschwindigkeit $u_{\mathrm{r}}$ herangezogen, kann der Ansatz

$$
\bar{h}=u_{\mathrm{r}}^{0,5} \cdot f(\beta)
$$

motiviert werden. Der Wärmeübergang wird nach Gleichung 6.5 als Produkt aus $u_{\mathrm{r}}^{0,5}$ und einer Funktion $f$, die nur vom Aufprallwinkel $\beta$ abhängt, beschrieben.

Der Verlauf von $\bar{h} /\left(u_{\mathrm{r}}\right)^{0,5}$ in Abhängigkeit von $\beta$ ist in Abbildung 6.16 für alle gemessenen Betriebspunkte bei $\vartheta_{\mathrm{s}}=80^{\circ} \mathrm{C}$ dargestellt. Der bezogene Wärmeübergang weist eine starke Abhängigkeit von $\beta$ auf. Dabei wird zwischen anliegenden und abgelösten Düsenströmungen unterschieden. Als anliegende Strömungen werden solche mit $\operatorname{Re}_{d} \leq 2251$ angesehen, bei denen in den Messungen keine abgelöste Düsenströmung beobachtet wird. Bei diesen anliegenden Strömungen konnte festgestellt werden, dass der Aufprallwinkel, ab dem der Rückgang des Wärmeübergangs einsetzt, kleiner als der bei abgelösten Strömungen ist. Der Aufprallwinkel, ab dem dieser Rückgang auftritt, wird im Folgenden als kritischer Aufprallwinkel, $\beta_{\mathrm{cr}}$, bezeichnet.

Sowohl für anliegende als auch für abgelöste Düsenströmungen wird ein ansatzweise linearer Verlauf von $\bar{h} /\left(u_{\mathrm{r}}\right)^{0,5}$ bis zu $\beta_{\mathrm{cr}}$ beobachtet. Bei Überschreiten dieses Wertes ist eine stärkere Abnahme von $\bar{h} /\left(u_{\mathrm{r}}\right)^{0,5}$ festzustellen, die durch ein Polynom zweiten Grades beschrieben werden kann. Dieser charakteristische Verlauf ist auf die in Kapitel 6.2.1 beschriebenen Vorgänge zurückzuführen. Die kritischen Aufprallwinkel für anliegende und abgelöste Düsenströmungen, $\beta_{\mathrm{cr}, 1}$ und $\beta_{\mathrm{cr}, \mathrm{h}}$, unterscheiden sich. 


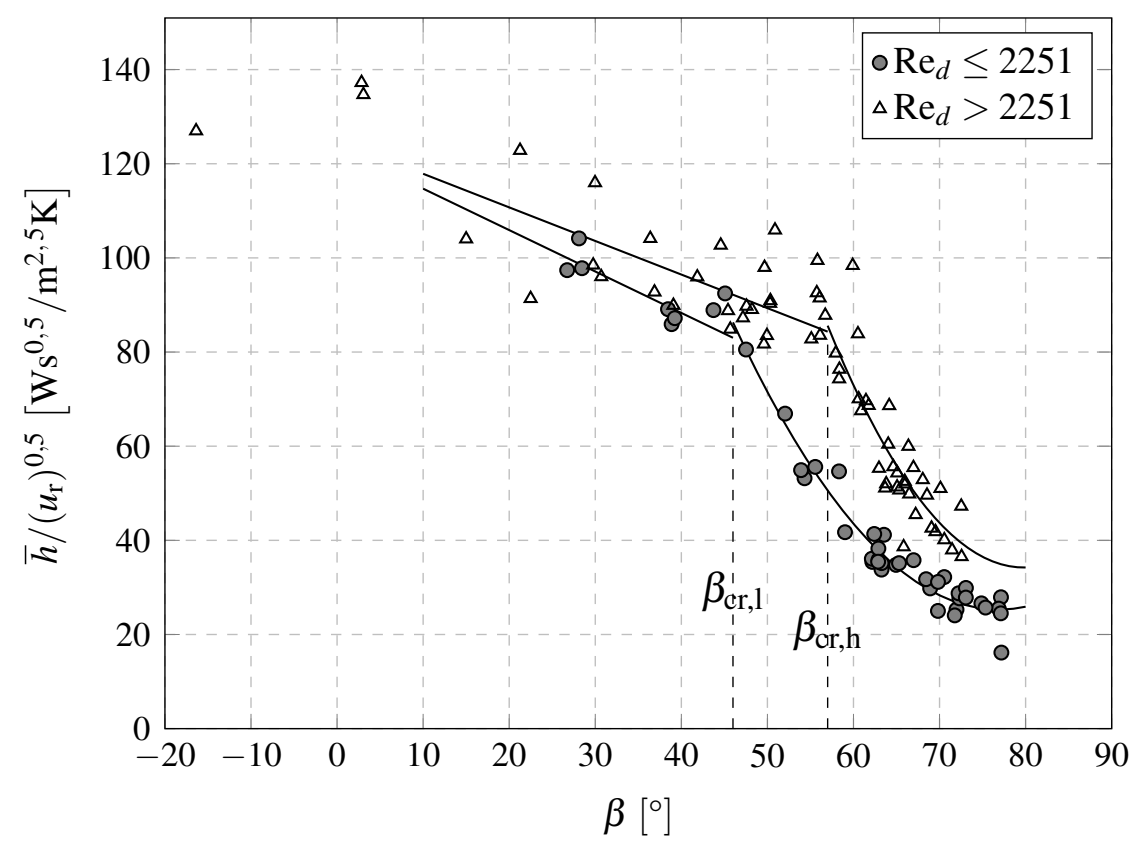

Abbildung 6.16: Werte des Ausdrucks $\bar{h} /\left(u_{\mathrm{r}}\right)^{0,5}$ in Abhängigkeit von $\beta$ für alle gemessenen Betriebspunkte bei $\vartheta_{\mathrm{s}}=80^{\circ} \mathrm{C}$

Unter Berücksichtigung der Form der Düsenströmung und Unterteilung in unter- und überkritische Aufprallwinkel können alle Datenpunkte durch den Ansatz aus Gleichung 6.5 gut wiedergegeben werden. Dazu wird für beide Formen der Düsenströmung jeweils ein unter- und überkritischer Bereich definiert, deren Grenzen durch die jeweiligen kritischen Aufprallwinkel definiert sind, mit

$$
\beta_{\mathrm{cr}, 1}=46^{\circ}
$$

für anliegende Düsenströmungen und

$$
\beta_{\mathrm{cr}, \mathrm{h}}=57^{\circ}
$$

für abgelöste Düsenströmungen. Der unterkritische Bereich $\beta<\beta_{\mathrm{cr}}$ wird dabei durch ein Polynom ersten Grades, der überkritische Bereich durch ein Polynom zweiten Grades abgebildet:

$$
\bar{h}=u_{\mathrm{r}}^{0,5} \cdot\left(p_{1} \beta^{2}+p_{2} \beta+p_{3}\right) .
$$

Durch eine Fehlerquadratminimierung können die Polynomkoeffizienten zu Gleichung 6.6 gefunden werden, die in Tabelle 6.2 aufgeführt sind.

Aus dem Vergleich der berechneten Wärmeübergangskoeffizienten $\bar{h}_{\mathrm{b}}$ mit den gemessenen Werten $\bar{h}$ in Abbildung 6.17 geht hervor, dass die Beschreibung des mittleren Wärmeübergangs durch Gleichung 6.6 und die Koeffizienten aus Tabelle 6.2 im Allgemeinen eine sehr gute Übereinstimmung mit den gemessenen Werten aufweist. Die Standardabweichung des Fehlers beträgt dabei $\sigma=10,7 \%$.

Der durch Gleichung 6.6 beschriebene Zusammenhang unterliegt der Einschränkung, dass er nur für die untersuchte Düsenkonfiguration gültig ist. Eine Übertragung des Wärmeübergangs 
Tabelle 6.2: Polynomkoeffizienten des stückweisen Polynomansatzes nach Gleichung 6.6

\begin{tabular}{lll}
\hline Düsenströmung & $\beta \leq \beta_{\mathrm{cr}}$ & $\beta>\beta_{\mathrm{cr}}$ \\
\hline \multirow{3}{*}{ anliegend } & $p_{1}=0$ & $p_{1}=0,06391$ \\
& $p_{2}=-0,8798$ & $p_{2}=-9,829$ \\
& $p_{3}=123,5$ & $p_{3}=403,2$ \\
\hline \multirow{3}{*}{ abgelöst } & $p_{1}=0$ & $p_{1}=0,09888$ \\
& $p_{2}=-0,7135$ & $p_{2}=-15,78$ \\
& $p_{3}=125$ & $p_{3}=663,8$ \\
\hline
\end{tabular}

im Bereich der Stauströmung für geänderte Düsendurchmesser ist denkbar. Die Auswirkungen von Veränderungen des Düsendurchmessers oder der Abstände zwischen den Öldüsen werden jedoch nicht durch die im Stand der Wissenschaft wiedergegebenen Korrelationen erfasst. Diese Einschränkung kann aufgehoben werden, sobald experimentelle oder auch numerische Daten mit entsprechend geänderten Düsenkonfigurationen verfügbar sind.

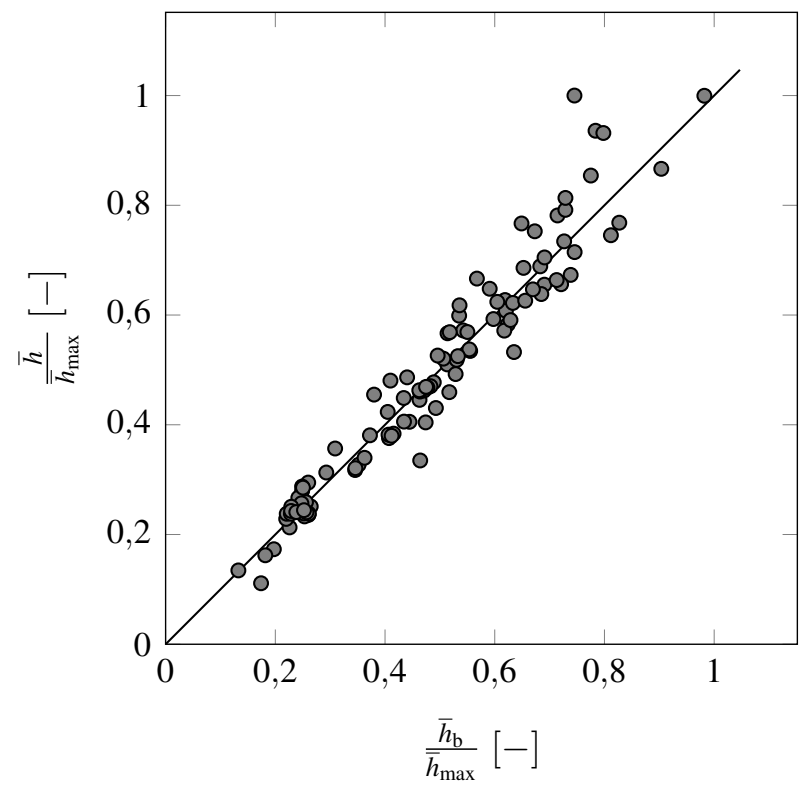

Abbildung 6.17: Vergleich der gemessenen und der durch die Korrelation berechneten mittleren Wärmeübergangskoeffizienten, jeweils bezogen auf den maximalen gemessenen mittleren Wärmeübergangskoeffizienten $\bar{h}_{\text {max }}$

Die getroffene Aufteilung der Düsenströmung in anliegende und abgelöste Bereiche ist notwendig, da die Berechnung von $\Sigma$ und $\beta$ bei abgelösten Strömungen durch die geänderte Strahlgeschwindigkeit fehlerhaft ist. Die tatsächliche Strahlgeschwindigkeit wird im Rahmen dieser Arbeit nicht gemessen. Unter Verwendung gemessener Strahlgeschwindigkeiten und dadurch korrigierter Berechnungen besteht die Möglichkeit, dass die Verläufe beider Bereiche zusammenfallen und somit nur eine Aufteilung in Bereiche unter- und überkritischer Aufprallwinkel getroffen werden muss. Eine Berechnung der Strömungsgeschwindigkeit ausschließlich 
anhand der vorliegenden Daten zur Düse und der Strömung ist nicht möglich. 


\section{Zusammenfassung}

Die Verbesserung von Triebwerken hinsichtlich ihres Treibstoffverbrauchs ist durch jahrzehntelange Forschung weit vorangeschritten. Klassische Optimierungspotentiale sind dadurch weitestgehend ausgeschöpft. Weitere, inkrementelle Wirkungsgradsteigerungen in diesen Bereichen, etwa durch verbesserte Materialien oder Kühlmethoden, sind daher enorm kostenintensiv. Das Konzept des Getriebefantriebwerks hat das Potential, einen disruptiven Entwicklungsfortschritt auszulösen. Jedoch stellt das benötigte Höchstleistungsgetriebe eine neue und hochkomplexe Komponente dar, deren Entwicklung eine technologische Herausforderung ist. Das zur Umsetzung des Konzepts notwendige Getriebe muss hohen und sehr gegensätzlichen Anforderungen genügen. Die Leistungsdichte muss durch die Forderung nach geringem Gewicht und kleinem Bauraum extrem hoch sein. Als Konsequenz ist die Wärmestromdichte, die durch Reibungsvorgänge im Zahnradeingriff entsteht, hoch. Eine wesentliche Herausforderung besteht in der Kühlung der hochbelasteten Zahnräder.

Das Ziel dieser Arbeit war, ein tiefgreifendes Verständnis der Zahnradkühlung durch Öleinspritzung zu gewinnen und so einen Beitrag zur Entwicklung von Höchstleistungsgetrieben zu leisten. Dazu wurde der Einfluss der Betriebsparameter Drehzahl, Ölvolumenstrom, Düsenwinkel und Öltemperatur auf den ortsaufgelösten Wärmeübergang am Zahnrad analysiert. Einerseits wurden dadurch die relevanten Zusammenhänge aufgedeckt, die den Wärmeübergang beeinflussen und durch deren Kenntnis eine effiziente Auslegung des Kühlsystems erst möglich wird. Andererseits wurde so eine umfangreiche Datenbasis mit exakt definierten Randbedingungen geschaffen, durch die in Zukunft numerische Modelle validiert werden können. Erst durch diese Datenbasis sind in Zukunft kostensparende CFD-Simulationen des Öl-Zahnrad-Wärmeübergangs möglich.

Bisherige Arbeiten und Prüfstände liefern keine ortsaufgelösten Messdaten des Wärmeübergangs. Zusätzlich ist durch das gemeinsame Auftreten von Reibung und Kühlung die Bestimmung von Wärmeübergangskoeffizienten in diesen Arbeiten nur eingeschränkt möglich. Daher wurde in dieser Arbeit eine neue Methode entwickelt und vorgestellt, mit der der Wärmeübergang an einem Zahnrad erstmals getrennt von Reibwärmeeinflüssen und ortsaufgelöst gemessen werden konnte. Durch die Analyse der Methode durch Monte-Carlo-Simulationen konnte deren hohe Messgenauigkeit nachgewiesen werden. Durch die Daten der Monte-Carlo-Simulationen konnte zusätzlich die Robustheit der ortsaufgelösten Messung bewiesen werden.

Mit einem neu entwickelten und am Institut für Thermische Strömungsmaschinen gefertigten Prüfstand wurde der Wärmeübergang am Zahnrad für eine Konfiguration der Einspritzkühlung und eine Vielzahl von Betriebspunkten untersucht. Dabei wurde ein komplexer Zusammenhang zwischen den Betriebsparametern und dem Wärmeübergang beobachtet, der durch bisherige Arbeiten nicht abgebildet wird. Es zeigte sich die Existenz einer kritischen Drehzahl, bei deren Überschreitung ein deutlicher Rückgang des Wärmeübergangs zu verzeichnen ist. Durch Verwendung eines kinematischen Modells des Ölaufpralls konnten die beobachteten Zusammenhänge erklärt werden. Der Aufprallwinkel, unter dem der Ölstrahl auf die Zahnflanke auftrifft, hat dabei eine herausragende Bedeutung für den zu erwartenden mittleren Wärmeübergang. Durch die identifizierten Zusammenhänge wurde eine Korrelation abgeleitet, die den mittleren Wär- 
meübergang im bisher erfassten Parameterbereich der Einspritzkühlung durch einen einfachen mathematischen Ansatz in sehr guter Näherung abbildet.

Durch die Thermalmodellierung des Zahnrads unter Berücksichtigung der Reibung kann gezeigt werden, dass nicht nur der mittlere Wärmeübergang, sondern auch seine örtliche Verteilung zur Einhaltung von Temperaturgrenzen von Bedeutung ist (Bauer (2018)). Daten örtlich aufgelöster Messungen des Wärmeübergangs sind durch die vorliegende Arbeit erstmalig und für einen weiten Parameterbereich verfügbar. Dies versetzt den Entwickler in die Lage, eine ausreichende Kühlung der Zahnflanken sicherzustellen, ohne dabei Wirkungsgradverluste durch zu hohe eingesetzte Ölvolumenströme zu riskieren. Dadurch, dass somit die Versorgungs- und Übertragungssysteme im Ölkreislauf des Triebwerks kleiner dimensioniert werden können, stellt dies einen wertvollen Baustein für die Entwicklung eines Höchstleistungsgetriebes für das Getriebefantriebwerk dar.

Um eine Basis für eine allgemeingültige Korrelation für den Wärmeübergang zu schaffen, muss der Wärmeübergang bei geänderten Geometrien untersucht werden. Durch die in dieser Arbeit entstandene Methode sind die dafür notwendigen Grundlagen geschaffen worden. Schrägverzahnung, die Zähnezahl und der Modul sind Einflussgrößen, deren Wirkung bisher nicht bekannt ist und in zukünftigen Untersuchungen bestimmt werden muss.

Das grundlagenorientierte Wissen zur instationären Prallkühlung bewegter Oberflächen ist sehr begrenzt. Die Beschreibung des Öl-Zahnrad-Wärmeübergangs als Modell aus Teilprozessen ist bisher nicht möglich, da diese Teilprozesse in der Literatur nur unvollständig beschrieben sind. Wenn durch zukünftige Arbeiten das Grundlagenwissen erweitert wird, kann die Modellbeschreibung des Öl-Zahnrad-Wärmeübergangs möglich werden. Durch die umfangreiche Datenbasis, die mit dieser Arbeit vorliegt, können solche Modelle validiert werden. Die Übertragung der Ergebnisse dieser Arbeit auf Zahnräder mit anderer Geometrie, aber auch weitere technische Fragestellungen im Kontext der Prallkühlung, kann dadurch ermöglicht werden. 


\section{Anhang}

\section{A.1 Gemessene Betriebspunkte und Wärmeübergangsverteilungen}

\section{A.1.1 Tabellarische Darstellung der Parameter}

Tabelle A.1: Parameter und $\overline{\mathrm{Nu}}^{*}$ der gemessenen Betriebspunkte

\begin{tabular}{|c|c|c|c|c|c|}
\hline BP & $\begin{array}{l}n \\
1 / \min \end{array}$ & $\begin{array}{l}\alpha \\
\circ\end{array}$ & $\begin{array}{l}\vartheta_{\mathrm{s}} \\
{ }^{\circ} \mathrm{C}\end{array}$ & $\begin{array}{l}\dot{V} \\
1 / \min \end{array}$ & $\begin{array}{l}\overline{\mathbf{N u}}^{*} \\
-\end{array}$ \\
\hline BP1 & 1988 & 10,78 & 76,46 & 1,56 & 0,140 \\
\hline BP2 & 1988 & 10,76 & 78,04 & 2,45 & 0,219 \\
\hline BP3 & 1988 & 10,77 & 79,29 & 3,28 & 0,300 \\
\hline BP4 & 1988 & 23,49 & 79,57 & 3,28 & 0,298 \\
\hline BP5 & 1988 & 30,06 & 79,71 & 3,28 & 0,295 \\
\hline BP6 & 1988 & $-0,07$ & 79,85 & 3,28 & 0,321 \\
\hline BP7 & 2965 & 11,27 & 80,53 & 3,26 & 0,269 \\
\hline BP8 & 2804 & 12,72 & 68,82 & 3,9 & 0,286 \\
\hline BP9 & 2804 & 11,74 & 71,02 & 4,38 & 0,330 \\
\hline BP10 & 2804 & 11,79 & 71,92 & 4,59 & 0,441 \\
\hline BP11 & 2804 & 11,77 & 72,6 & 5,22 & 0,620 \\
\hline BP12 & 2804 & 11,8 & 73,34 & 6,86 & 0,823 \\
\hline BP13 & 3099 & 11,77 & 75,12 & 4,58 & 0,423 \\
\hline BP14 & 1995 & 11,79 & 76,27 & 3,32 & 0,318 \\
\hline BP15 & 1998 & 20,33 & 77,51 & 2,21 & 0,205 \\
\hline BP16 & 2805 & 20,21 & 78,2 & 6,83 & 0,852 \\
\hline BP17 & 3101 & 20,21 & 79,08 & 8,36 & 0,943 \\
\hline BP18 & 3785 & 20,29 & 80,05 & 7,14 & 0,904 \\
\hline BP19 & 5030 & 19,53 & 81,43 & 7,09 & 0,677 \\
\hline BP20 & 896 & 19,93 & 83,02 & 5,17 & 0,732 \\
\hline BP21 & 897 & 20,12 & 82,47 & 3,71 & 0,646 \\
\hline BP22 & 896 & 20,17 & 80,98 & 2,54 & 0,536 \\
\hline BP23 & 896 & 19,97 & 78,46 & 1,14 & 0,170 \\
\hline BP24 & 1568 & 30,98 & 78,4 & 2,85 & 0,301 \\
\hline BP25 & 3783 & 19,57 & 79,55 & 10,33 & 1,096 \\
\hline BP26 & 5054 & 19,29 & 80,74 & 10,28 & 1,265 \\
\hline BP27 & 1863 & 19,22 & 82,14 & 4,26 & 0,568 \\
\hline BP28 & 1998 & 19,51 & 80,05 & 3,19 & 0,302 \\
\hline BP29 & 1499 & 19,48 & 80,31 & 3,21 & 0,402 \\
\hline BP30 & 1002 & 19,49 & 80,47 & 3,23 & 0,564 \\
\hline BP31 & 2981 & 19,39 & 80,67 & 3,19 & 0,289 \\
\hline BP32 & 1996 & 19,4 & 81,9 & 4,84 & 0,738 \\
\hline BP33 & 991 & 19,32 & 82,13 & 4,84 & 0,674 \\
\hline BP34 & 1508 & 19,38 & 82,29 & 4,84 & 0,724 \\
\hline BP35 & 2994 & 19,27 & 82,23 & 4,84 & 0,582 \\
\hline BP36 & 2993 & 19,17 & 83,87 & 7,23 & 1,001 \\
\hline BP37 & 1996 & 19,02 & 84,21 & 7,21 & 0,955 \\
\hline BP38 & 1500 & 19,2 & 83,67 & 7,23 & 0,931 \\
\hline BP39 & 1003 & 19,21 & 84,04 & 7,23 & 0,895 \\
\hline
\end{tabular}




\begin{tabular}{|c|c|c|c|c|c|}
\hline BP & $\begin{array}{l}n \\
1 / \min \end{array}$ & $\begin{array}{l}\alpha \\
\circ\end{array}$ & $\begin{array}{l}\vartheta_{\mathrm{s}} \\
{ }^{\circ} \mathrm{C}\end{array}$ & $\begin{array}{l}\dot{V} \\
1 / \min \end{array}$ & $\begin{array}{l}\overline{\mathbf{N u}}^{*} \\
-\end{array}$ \\
\hline BP40 & 1997 & 19,24 & 81,42 & 3,16 & 0,354 \\
\hline BP41 & 2993 & 30,5 & 81,51 & 4,69 & 0,512 \\
\hline BP42 & 2993 & 44,49 & 81,87 & 4,68 & 0,476 \\
\hline BP43 & 2993 & 19,94 & 81,98 & 4,68 & 0,545 \\
\hline BP44 & 2993 & 0,27 & 82,15 & 4,68 & 0,585 \\
\hline BP45 & 3000 & $-10,48$ & 82,27 & 4,68 & 0,616 \\
\hline BP46 & 2992 & -20 & 82,34 & 4,68 & 0,609 \\
\hline BP47 & 2992 & $-29,91$ & 82,35 & 4,68 & 0,576 \\
\hline BP48 & 2993 & 10,63 & 82,59 & 4,67 & 0,605 \\
\hline BP49 & 3985 & 20 & 82,62 & 4,68 & 0,484 \\
\hline BP50 & 3986 & 19,68 & 82,02 & 3,15 & 0,338 \\
\hline BP51 & 2992 & 19,43 & 85,51 & 9,51 & 1,225 \\
\hline BP52 & 1500 & 20,07 & 79,96 & 3,24 & 0,414 \\
\hline BP53 & 1002 & 20,08 & 80,48 & 3,24 & 0,583 \\
\hline BP54 & 1998 & 20,05 & 80,68 & 3,23 & 0,328 \\
\hline BP55 & 2499 & 20,07 & 80,83 & 3,24 & 0,303 \\
\hline BP56 & 3006 & 20,05 & 80,19 & 3,24 & 0,301 \\
\hline BP57 & 3501 & 20,07 & 80,01 & 3,23 & 0,301 \\
\hline BP58 & 4002 & 20,05 & 80,28 & 3,23 & 0,309 \\
\hline BP59 & 748 & 20,08 & 80,62 & 3,23 & 0,595 \\
\hline BP60 & 1998 & 20,05 & 81,01 & 4,11 & 0,482 \\
\hline BP61 & 2496 & 20,02 & 81,1 & 4,11 & 0,396 \\
\hline BP62 & 2977 & 20,05 & 81,11 & 4,11 & 0,373 \\
\hline ВР63 & 1499 & 20,06 & 81,08 & 4,11 & 0,717 \\
\hline BP64 & 989 & 20,11 & 81,14 & 4,12 & 0,724 \\
\hline BP65 & 3503 & 20,03 & 81,07 & 4,11 & 0,364 \\
\hline BP66 & 4001 & 20,03 & 80,93 & 4,11 & 0,361 \\
\hline BP67 & 1997 & 20,07 & 81,45 & 4,77 & 0,794 \\
\hline BP68 & 2496 & 20,05 & 81,43 & 4,77 & 0,769 \\
\hline BP69 & 2750 & 20,04 & 81,5 & 4,77 & 0,680 \\
\hline BP70 & 3493 & 20,06 & 81,53 & 4,77 & 0,513 \\
\hline BP71 & 1488 & 20,07 & 80,94 & 5,96 & 0,829 \\
\hline BP72 & 1004 & 20,02 & 81,14 & 5,95 & 0,831 \\
\hline BP73 & 1998 & 20,04 & 81,33 & 5,95 & 0,872 \\
\hline BP74 & 2491 & 20,06 & 81,35 & 5,95 & 0,892 \\
\hline BP75 & 2993 & 20,03 & 81,32 & 5,95 & 0,930 \\
\hline BP76 & 3491 & 20,09 & 81,48 & 5,94 & 0,790 \\
\hline BP77 & 3988 & 20,03 & 81,59 & 5,94 & 0,656 \\
\hline BP78 & 1998 & 19,59 & 79,34 & 3,24 & 0,304 \\
\hline BP79 & 1998 & $-0,28$ & 79,69 & 3,25 & 0,325 \\
\hline BP80 & 1493 & $-0,31$ & 79,87 & 3,26 & 0,451 \\
\hline BP81 & 1002 & $-0,29$ & 80,02 & 3,25 & 0,586 \\
\hline BP82 & 747 & $-0,34$ & 80,1 & 3,25 & 0,592 \\
\hline BP83 & 2501 & $-0,35$ & 80,21 & 3,25 & 0,317 \\
\hline BP84 & 3002 & $-0,3$ & 80,22 & 3,23 & 0,307 \\
\hline BP85 & 3500 & $-0,3$ & 80,24 & 3,23 & 0,305 \\
\hline BP86 & 4001 & $-0,27$ & 80,25 & 3,23 & 0,309 \\
\hline BP87 & 1499 & $-0,19$ & 80,13 & 4,74 & 0,787 \\
\hline BP88 & 1997 & $-0,18$ & 80,29 & 4,74 & 0,808 \\
\hline BP89 & 2495 & $-0,22$ & 80,37 & 4,73 & 0,720 \\
\hline
\end{tabular}




\begin{tabular}{l|l|l|l|l|l} 
BP & $n$ & $\alpha$ & $\vartheta_{\mathbf{s}}$ & $\dot{V}$ & $\mathbf{N u}^{*}$ \\
& $1 /$ min & $\circ$ & ${ }^{\circ} \mathrm{C}$ & $1 /$ min & - \\
\hline BP90 & 2752 & $-0,21$ & 80,52 & 4,73 & 0,659 \\
BP91 & 3003 & $-0,23$ & 80,67 & 4,73 & 0,594 \\
BP92 & 3499 & $-0,22$ & 80,66 & 4,72 & 0,514 \\
BP93 & 4000 & $-0,18$ & 80,63 & 4,73 & 0,486 \\
BP94 & 1001 & $-0,2$ & 80,84 & 4,73 & 0,748 \\
BP95 & 1996 & $-0,23$ & 81,52 & 5,99 & 0,989 \\
BP96 & 2490 & $-0,19$ & 81,59 & 5,99 & 1,002 \\
BP97 & 3000 & $-0,2$ & 81,54 & 5,99 & 0,971 \\
BP98 & 3501 & $-0,22$ & 81,65 & 5,99 & 0,844 \\
BP99 & 3994 & $-0,2$ & 81,72 & 5,99 & 0,720 \\
BP100 & 4321 & $-0,22$ & 81,82 & 5,99 & 0,666 \\
BP101 & 1001 & $-0,22$ & 81,96 & 5,98 & 0,840 \\
BP102 & 1997 & 44,96 & 81,23 & 4,75 & 0,665 \\
BP103 & 2496 & 44,98 & 81,05 & 4,75 & 0,623 \\
BP104 & 3002 & 44,91 & 80,94 & 4,75 & 0,481 \\
BP105 & 3503 & 44,94 & 80,84 & 4,74 & 0,430 \\
BP106 & 3991 & 44,93 & 80,7 & 4,75 & 0,406 \\
BP107 & 1500 & 44,87 & 80,91 & 4,75 & 0,758 \\
BP108 & 1002 & 44,91 & 80,85 & 4,75 & 0,820 \\
BP109 & 1999 & 44,83 & 80,46 & 7,18 & 0,953 \\
BP110 & 2497 & 44,82 & 80,64 & 7,17 & 0,868 \\
BP111 & 2995 & 44,78 & 80,86 & 7,18 & 0,792 \\
BP112 & 3505 & 44,87 & 80,87 & 7,18 & 0,819 \\
BP113 & 4002 & 44,5 & 81,07 & 7,17 & 0,750 \\
BP114 & 1501 & 44,6 & 81,1 & 7,17 & 1,030 \\
BP115 & 1002 & 44,56 & 81,1 & 7,18 & 0,973 \\
BP116 & 1999 & $-0,2$ & 81,13 & 7,17 & 1,081 \\
BP117 & 2495 & $-0,2$ & 81,14 & 7,17 & 1,185 \\
BP118 & 2992 & $-0,19$ & 80,99 & 7,18 & 1,179 \\
BP119 & 1001 & 19,56 & 61,34 & 4,66 & 0,798 \\
BP120 & 1500 & 19,55 & 60,19 & 4,67 & 0,698 \\
BP121 & 1998 & 19,58 & 60,59 & 4,67 & 0,628 \\
BP122 & 1986 & 19,56 & 61,4 & 4,65 & 0,717 \\
BP123 & 2496 & 19,61 & 60,89 & 4,66 & 0,501 \\
BP124 & 2993 & 19,57 & 61,12 & 4,66 & 0,461 \\
BP125 & 3491 & 19,62 & 61,16 & 4,65 & 0,467 \\
BP126 & 3986 & 19,54 & 61,13 & 4,66 & 0,495 \\
BP127 & 3994 & $-0,7$ & 78,38 & 6,19 & 0,781 \\
BP128 & 3684 & $-0,21$ & 79,63 & 7,54 & 1,265 \\
BP129 & 3998 & 0,24 & 79,86 & 7,07 & 0,980
\end{tabular}

\section{A.1.2 Ortsaufgelöste Wärmeübergangskoeffizienten}




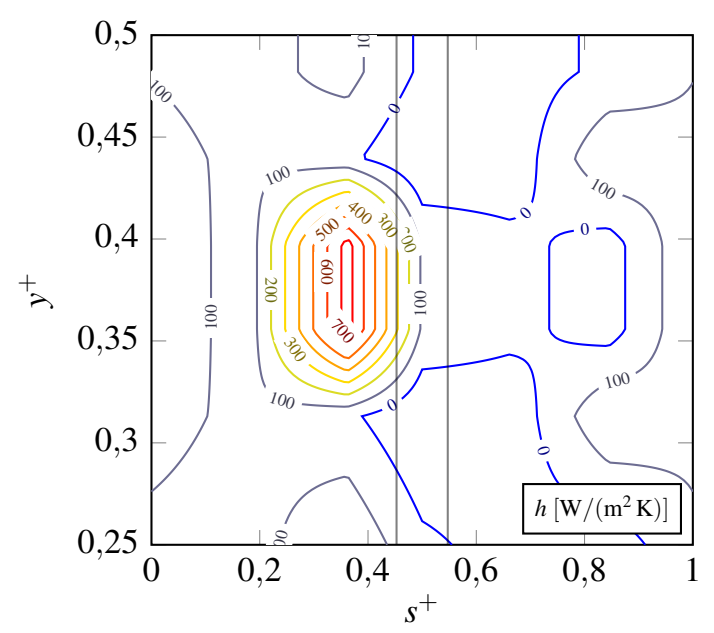

(a) BP1

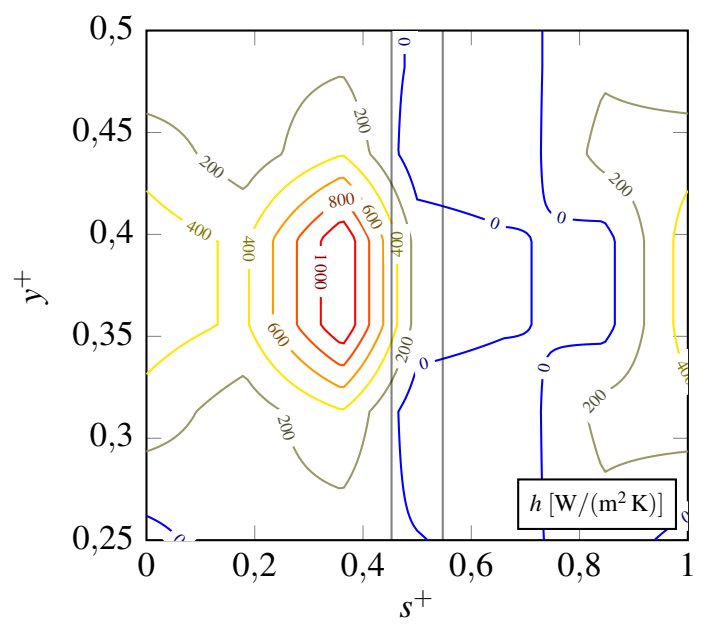

(c) BP3

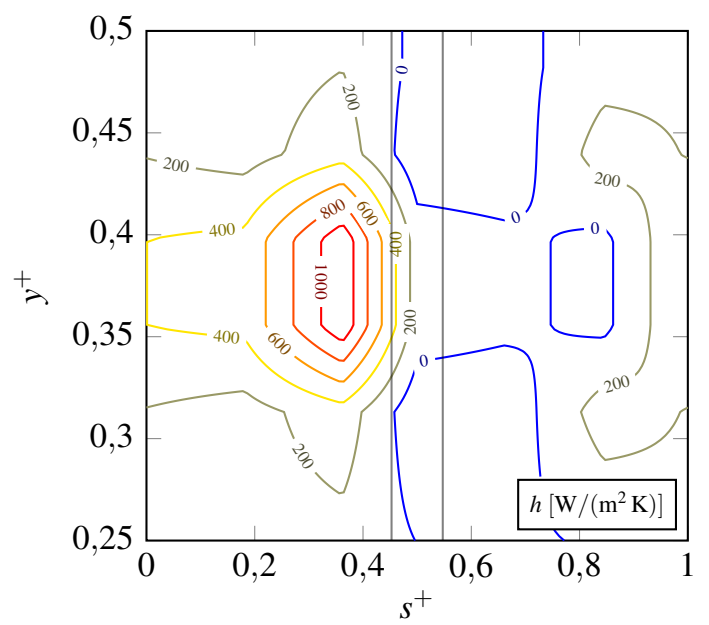

(e) BP5

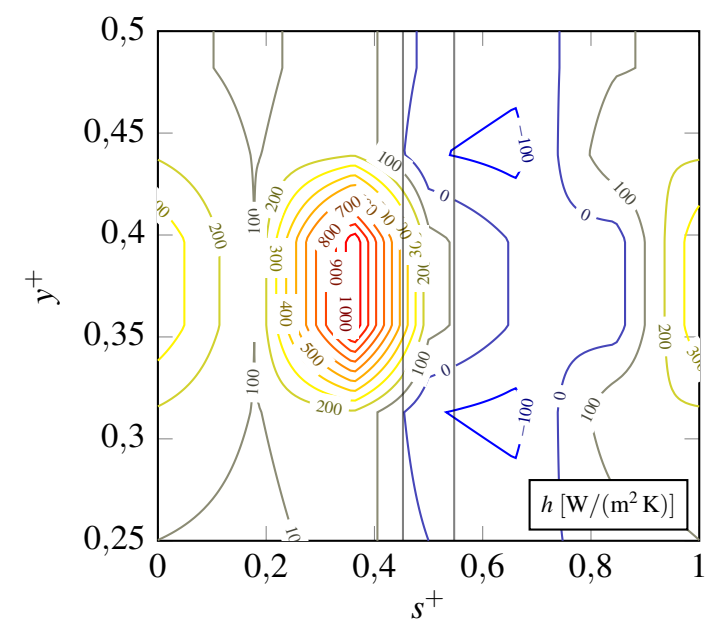

(b) BP2

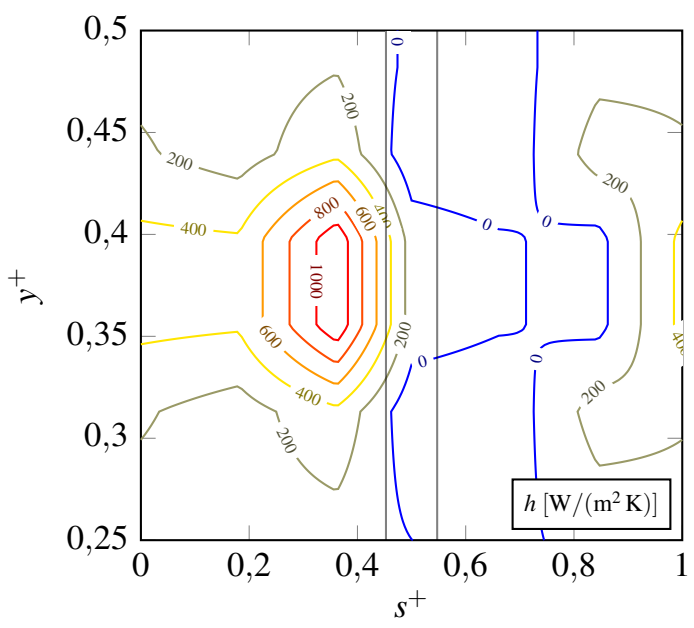

(d) BP4

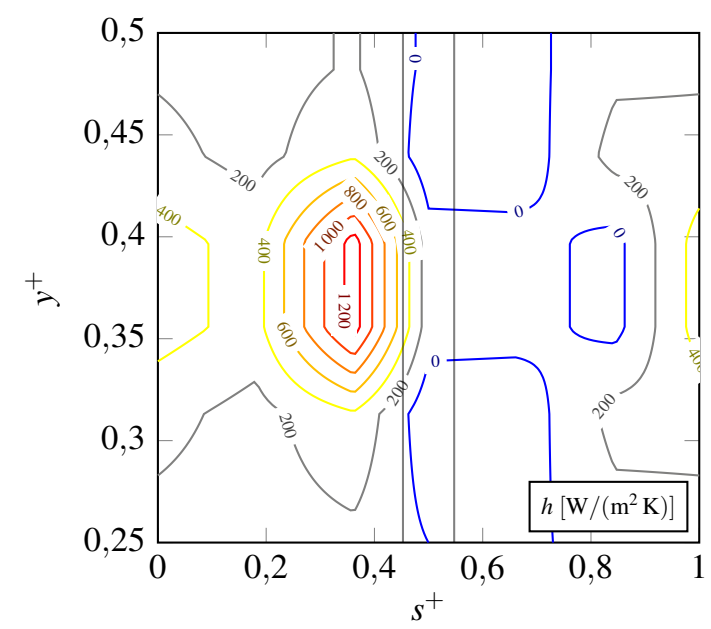

(f) BP6

Abbildung A.1: Ortsaufgelöste Wärmeübergangsmessungen für BP1 bis BP6 


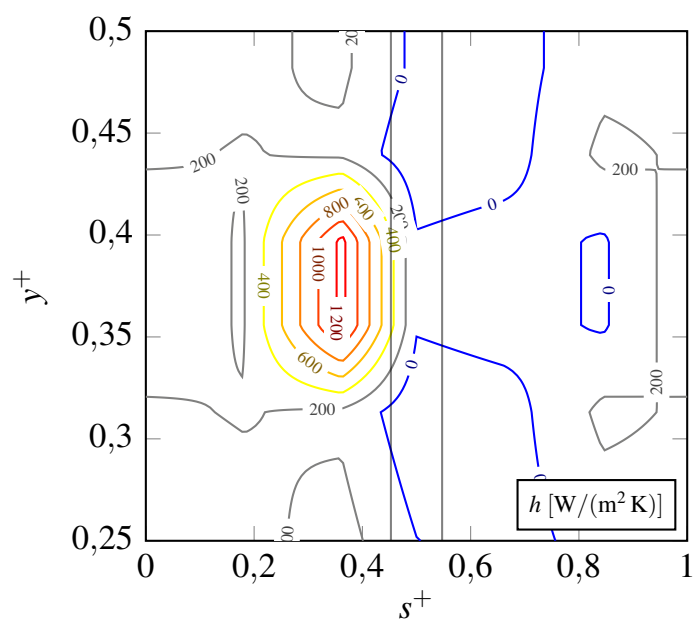

(a) BP7

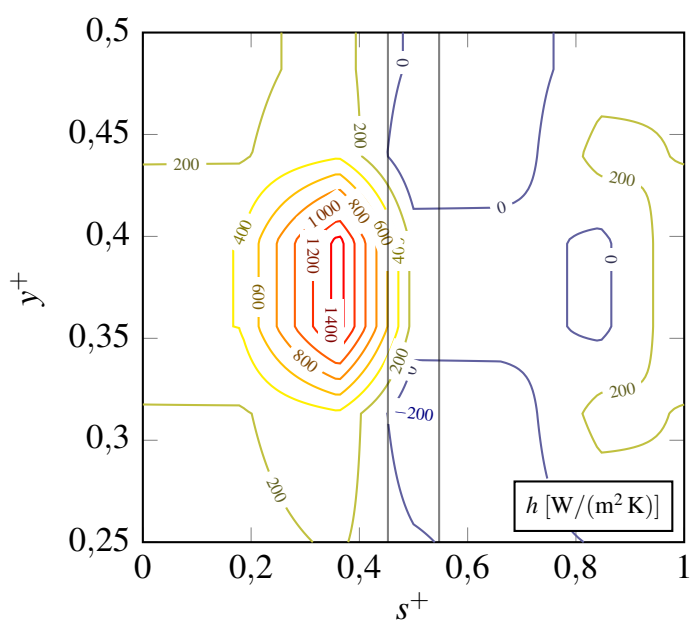

(c) BP9

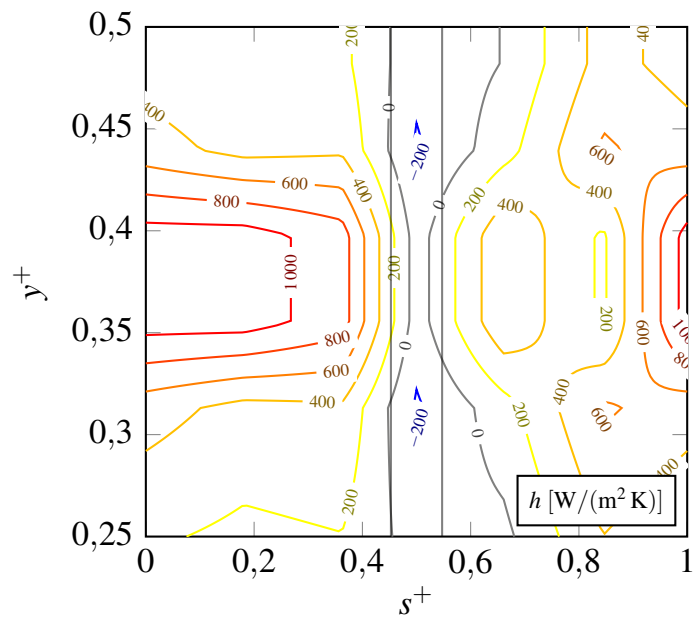

(e) BP11

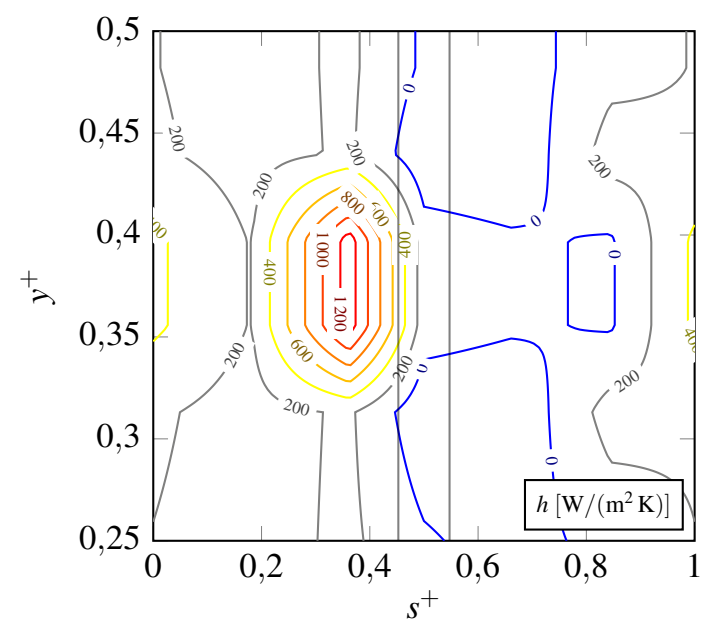

(b) BP8

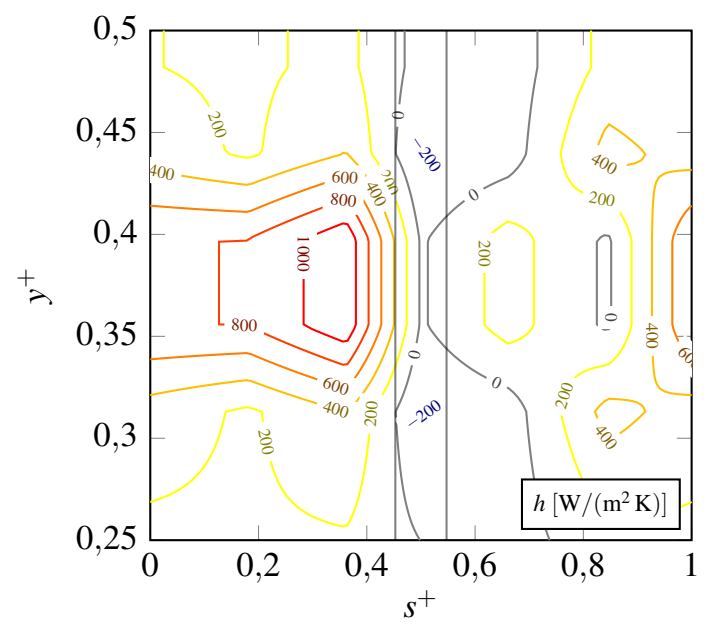

(d) BP10

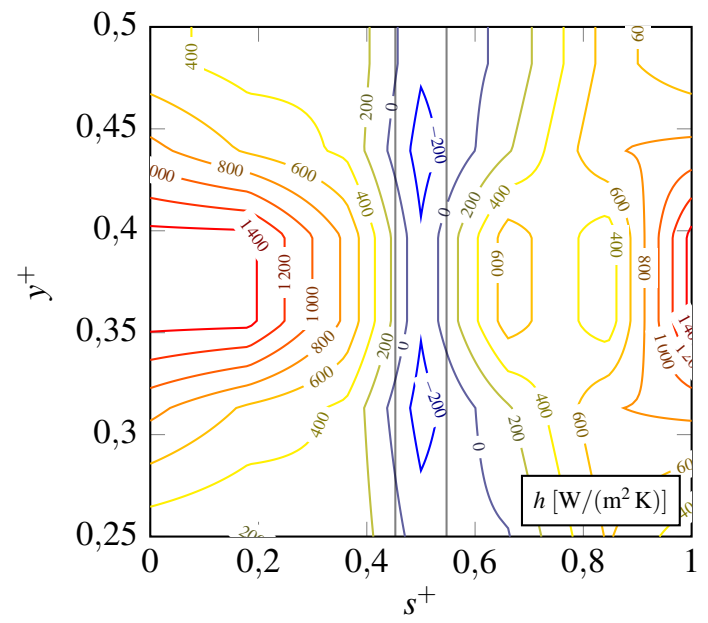

(f) BP12

Abbildung A.2: Ortsaufgelöste Wärmeübergangsmessungen für BP7 bis BP12 


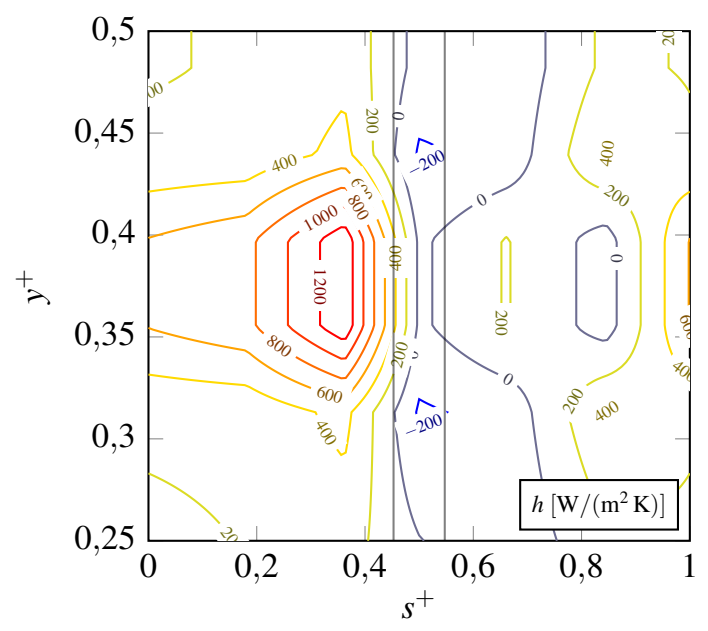

(a) BP13

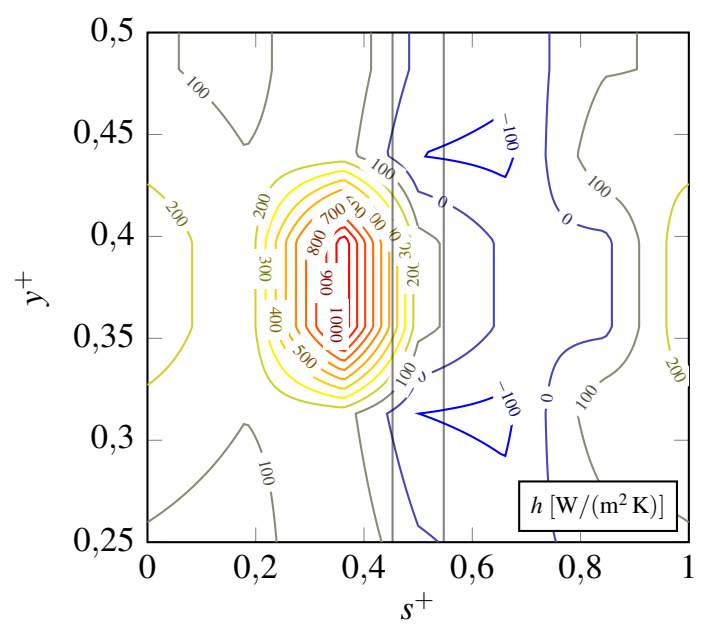

(c) BP15

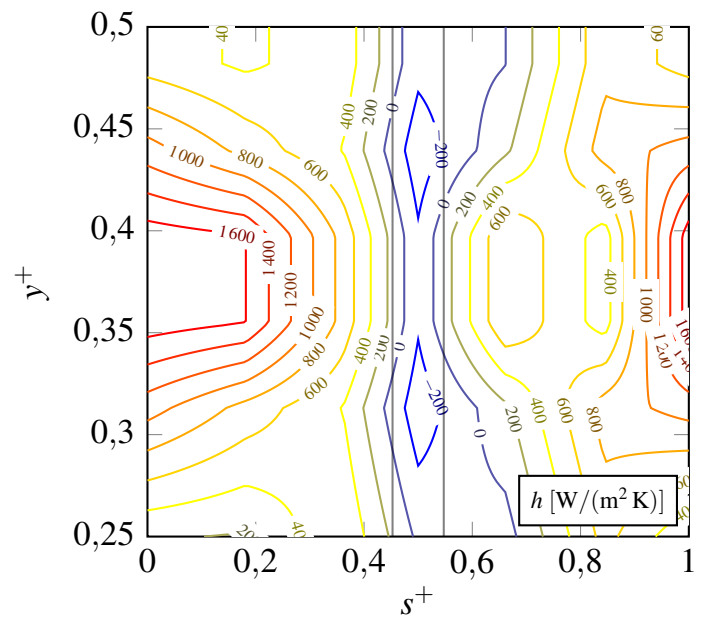

(e) BP17

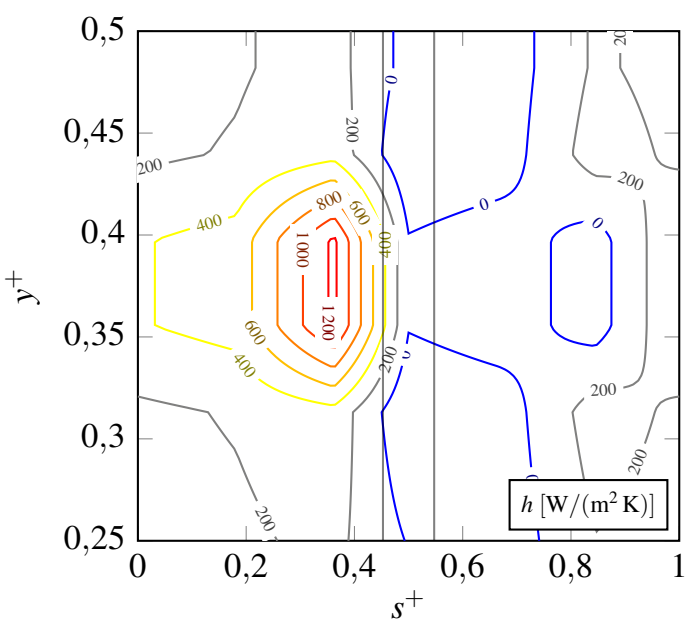

(b) BP14

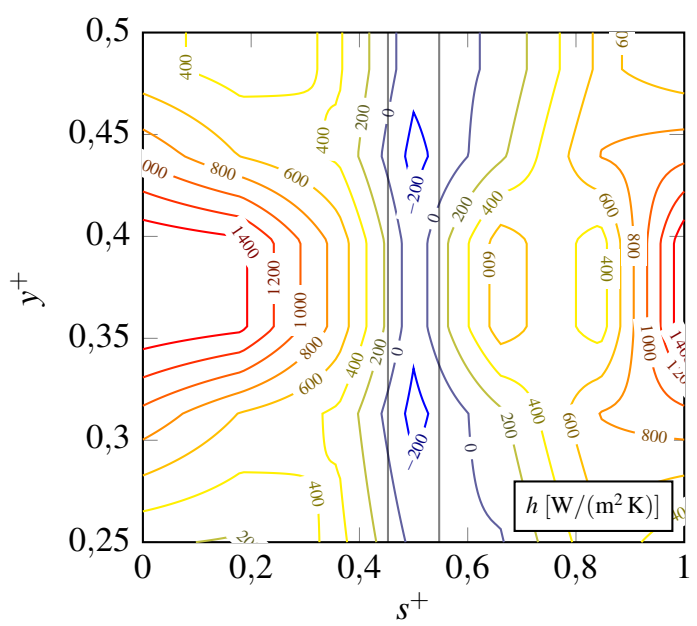

(d) BP16

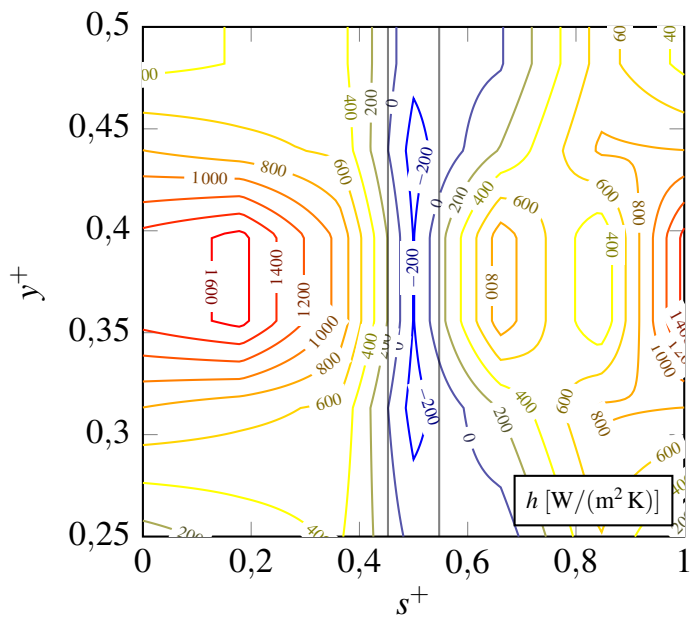

(f) BP18

Abbildung A.3: Ortsaufgelöste Wärmeübergangsmessungen für BP13 bis BP18 


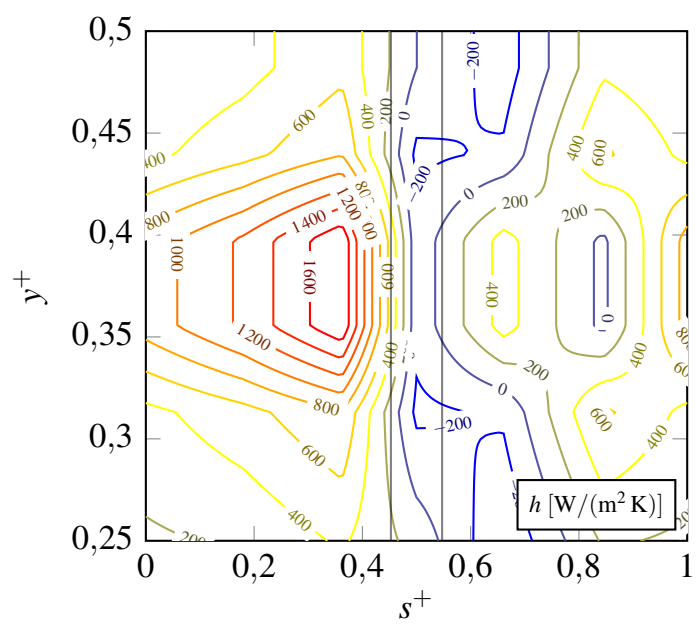

(a) BP19

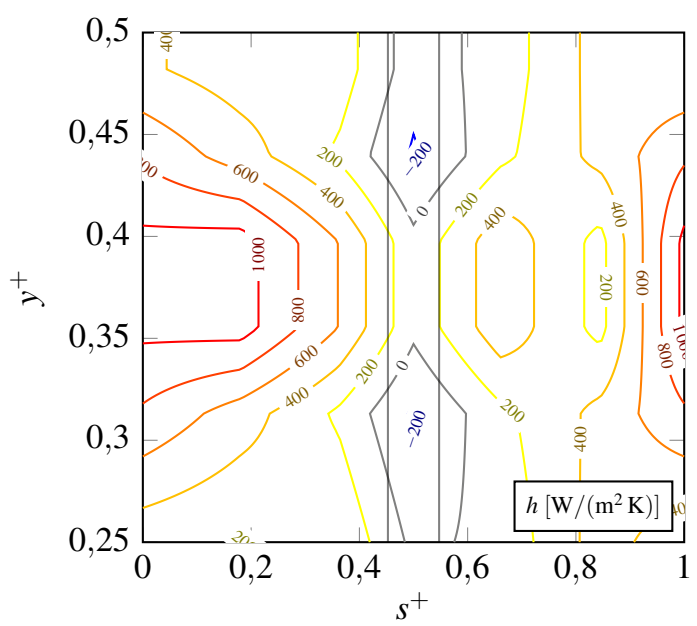

(c) BP21

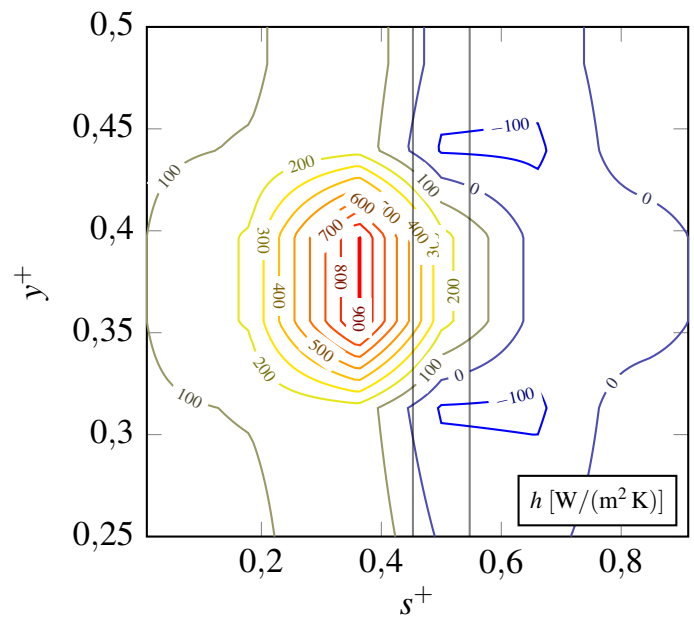

(e) BP23

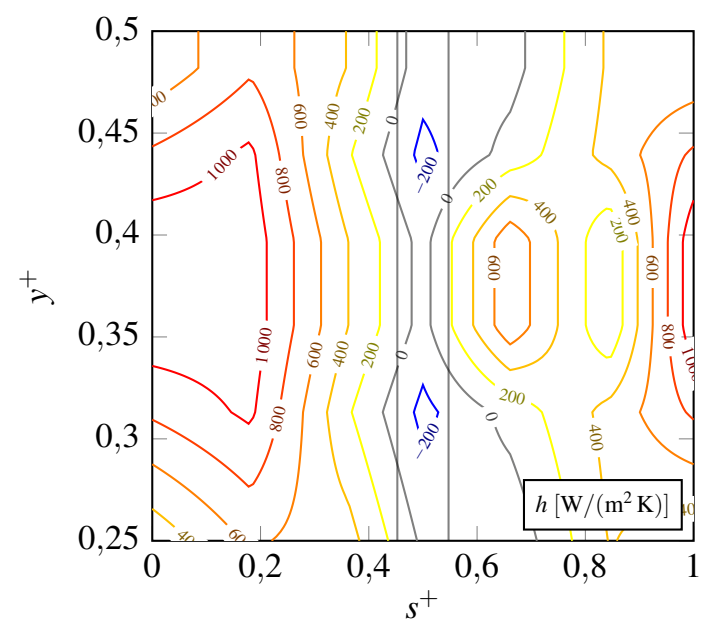

(b) BP20

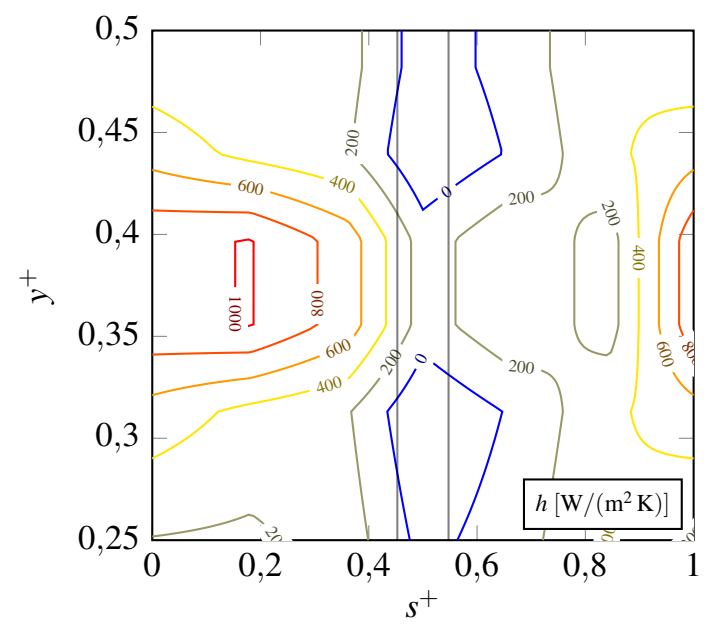

(d) BP22

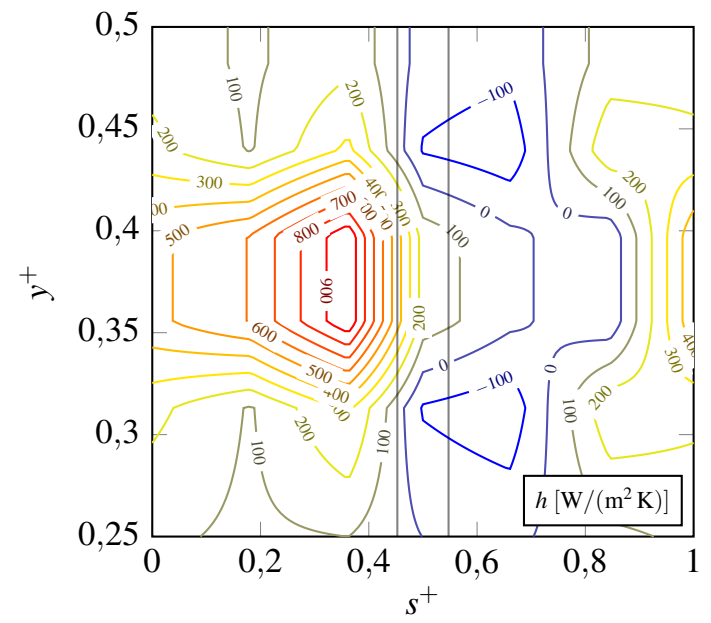

(f) BP24

Abbildung A.4: Ortsaufgelöste Wärmeübergangsmessungen für BP19 bis BP24 


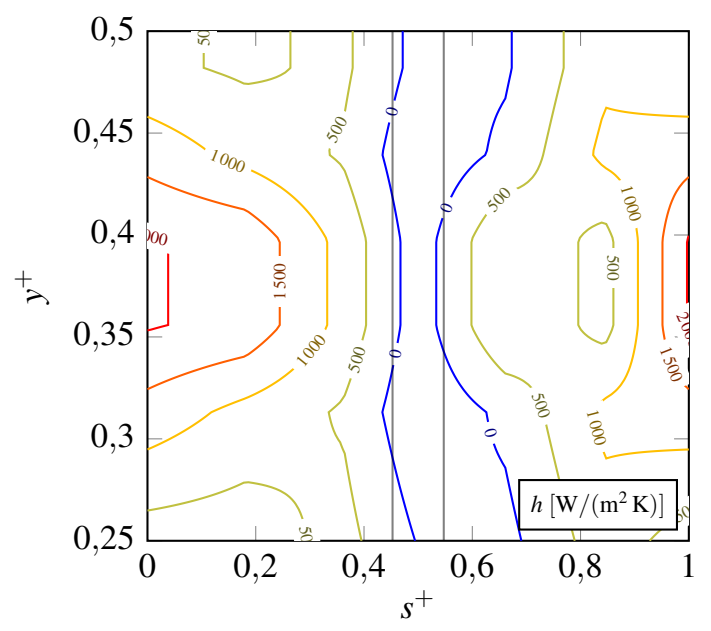

(a) BP25

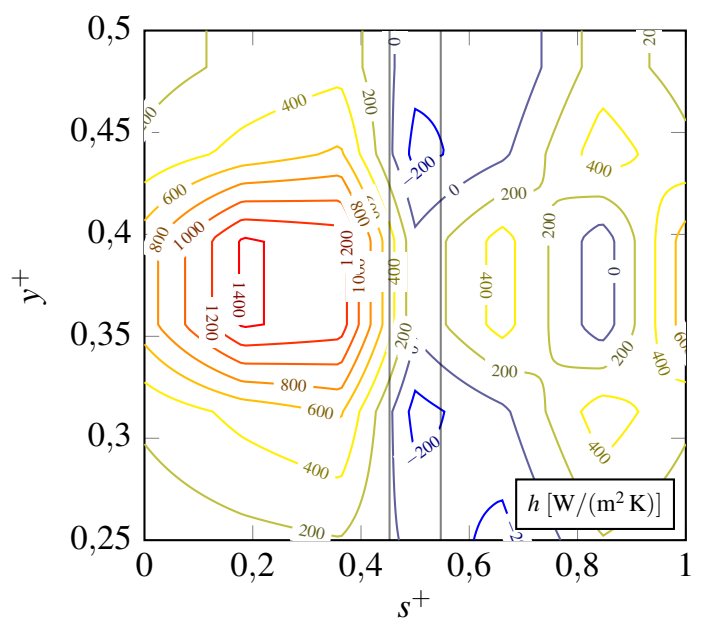

(c) BP27

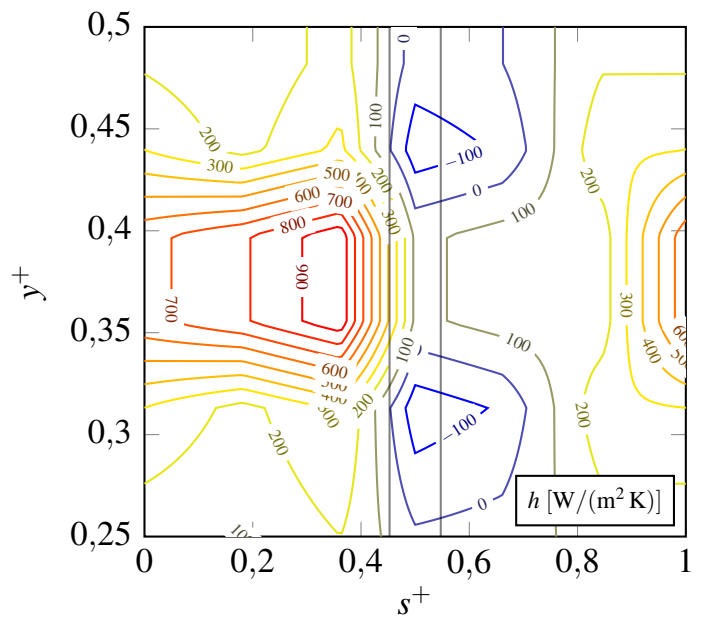

(e) BP29

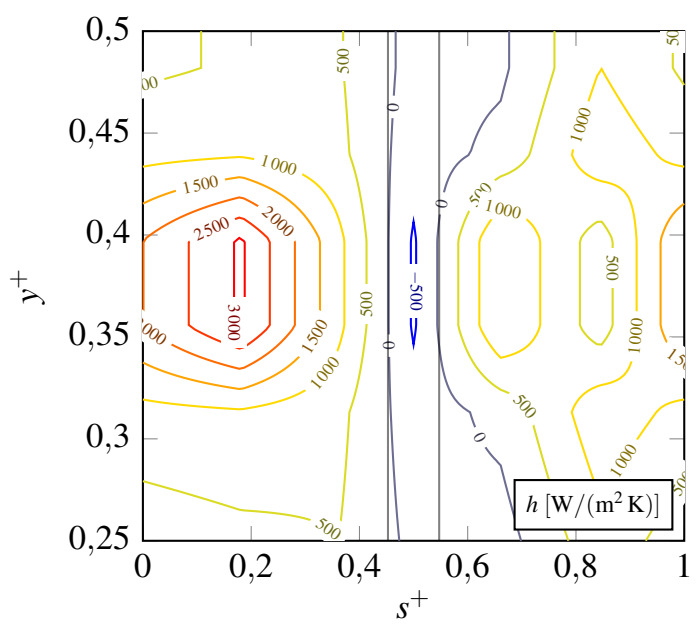

(b) BP26

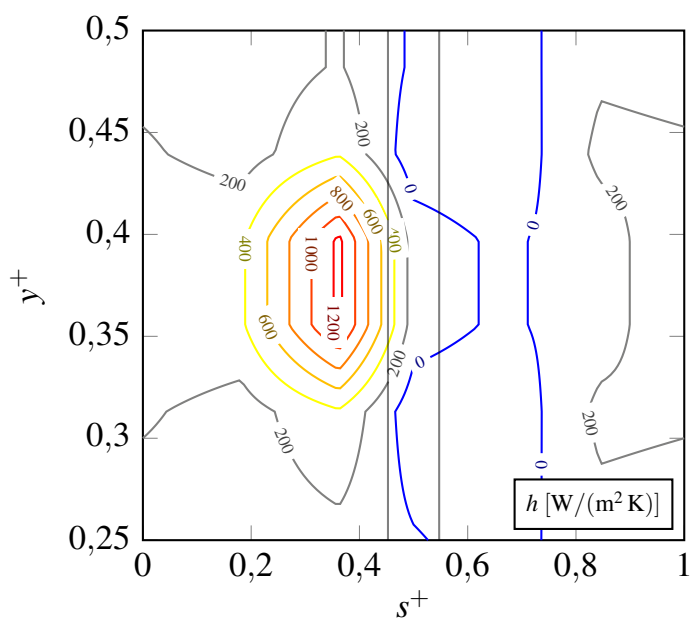

(d) BP28

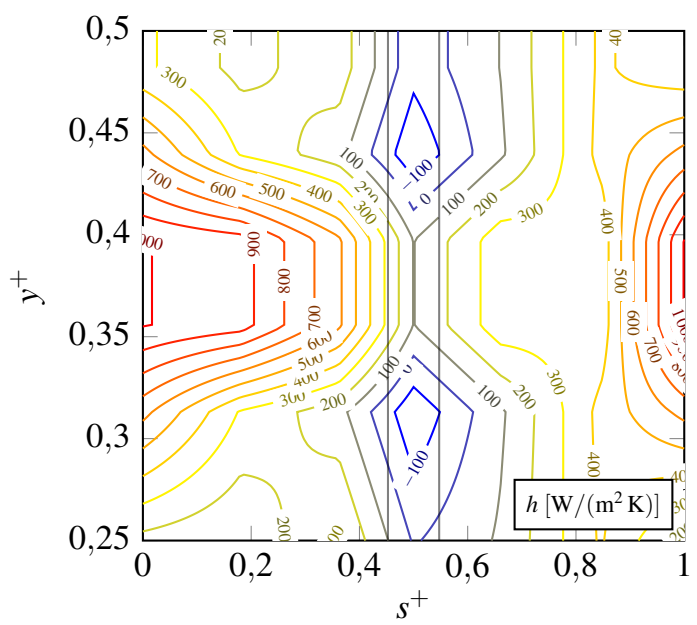

(f) BP30

Abbildung A.5: Ortsaufgelöste Wärmeübergangsmessungen für BP25 bis BP30 


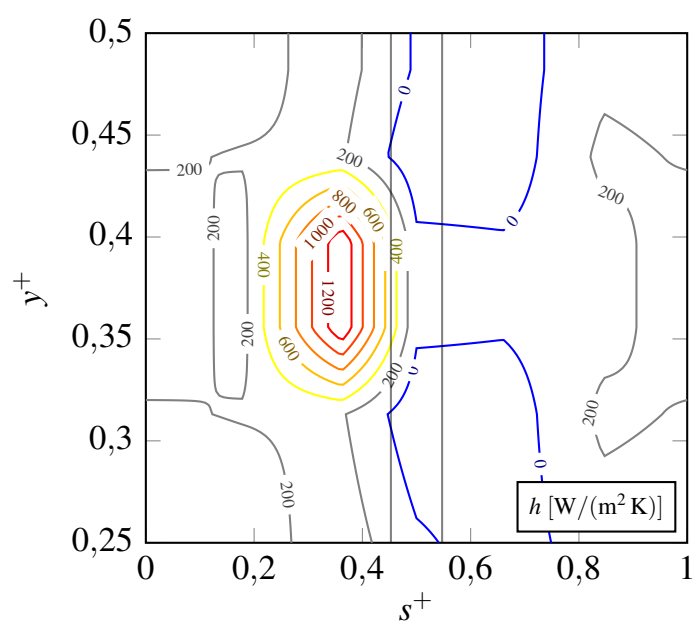

(a) BP31

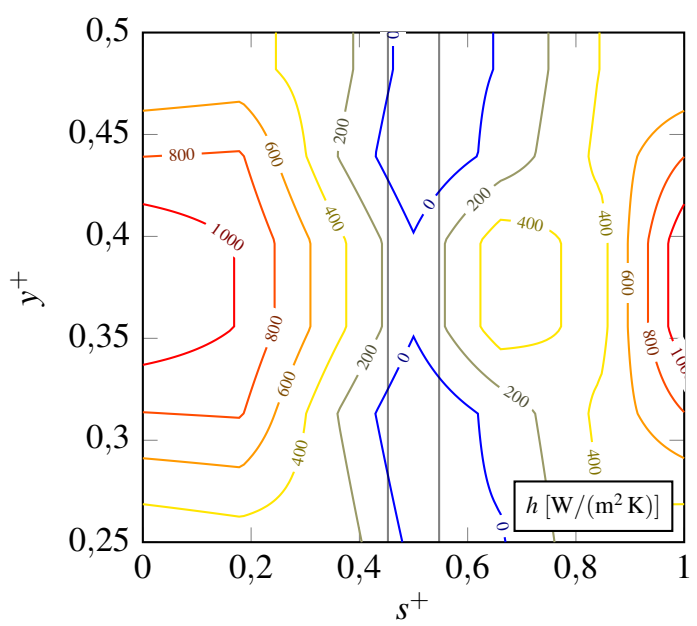

(c) BP33

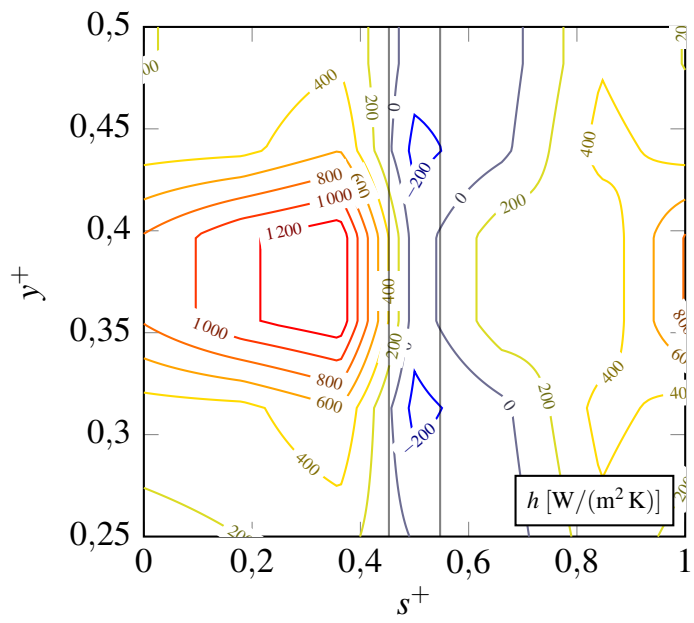

(e) BP35

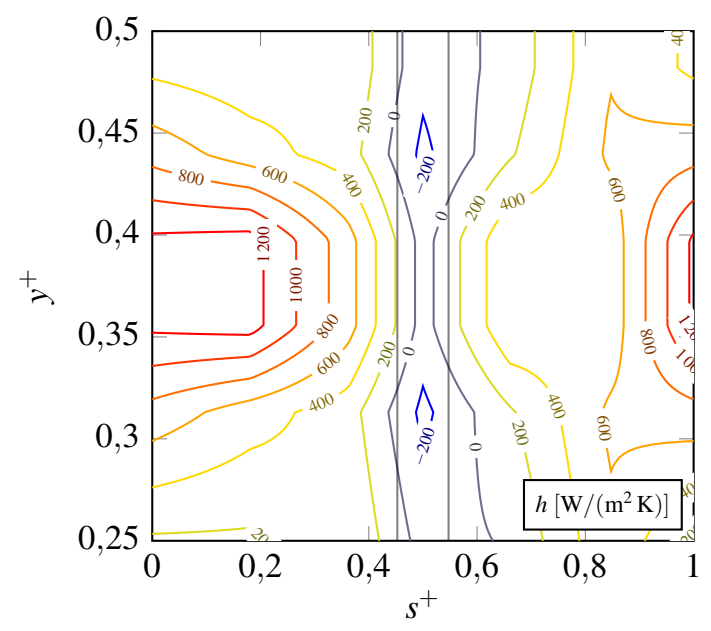

(b) BP32

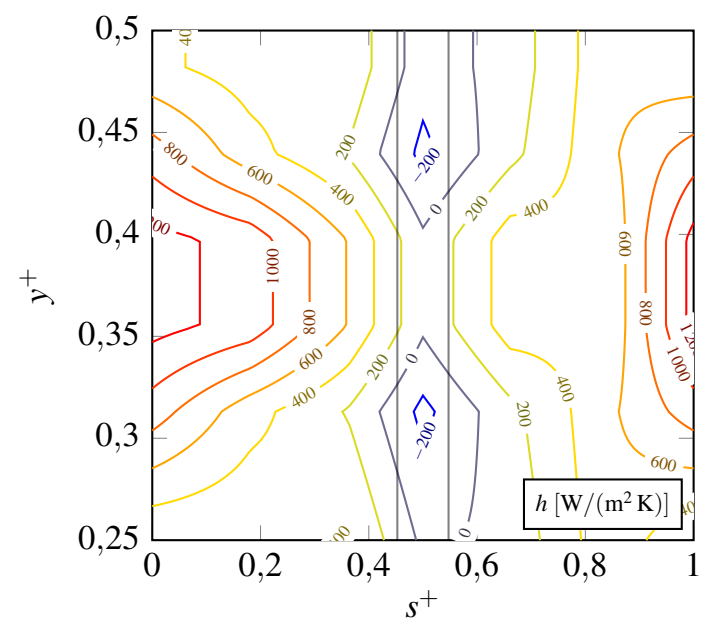

(d) BP34

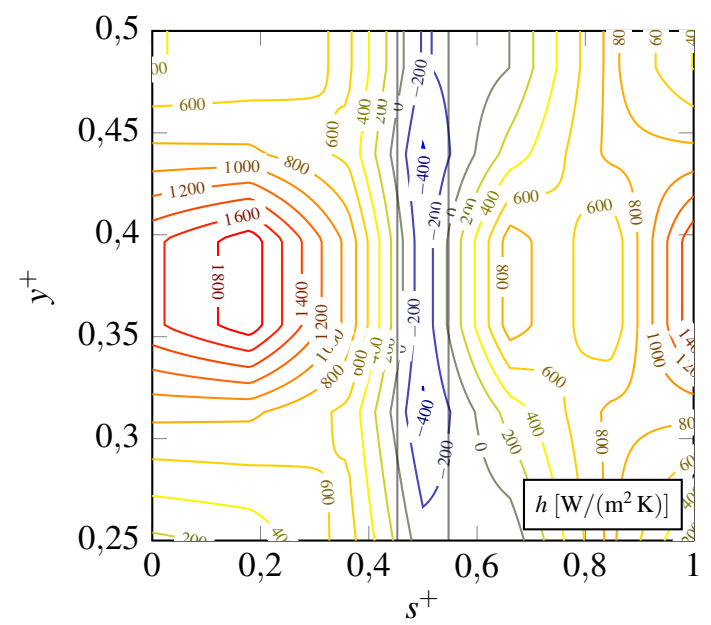

(f) BP36

Abbildung A.6: Ortsaufgelöste Wärmeübergangsmessungen für BP31 bis BP36 


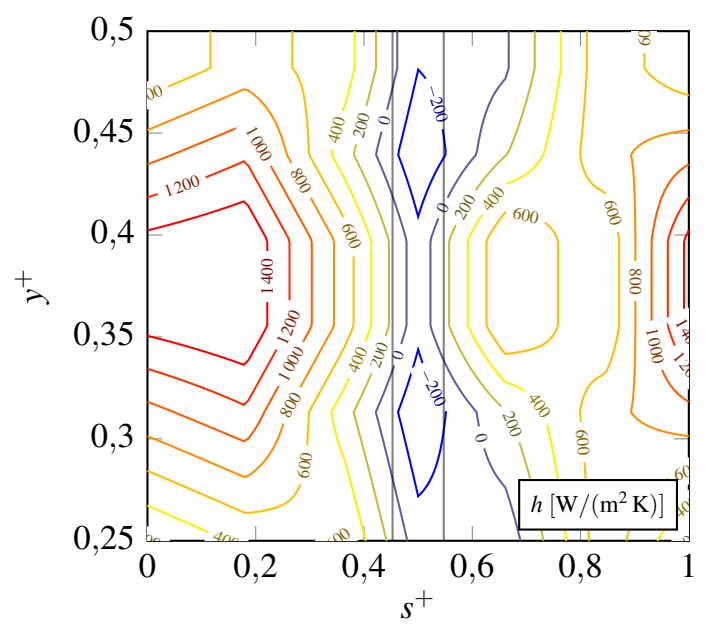

(a) BP37

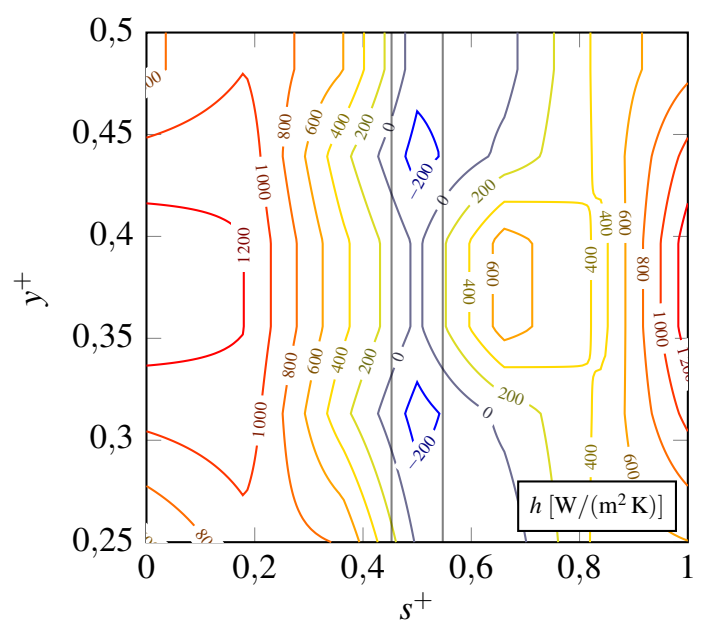

(c) BP39

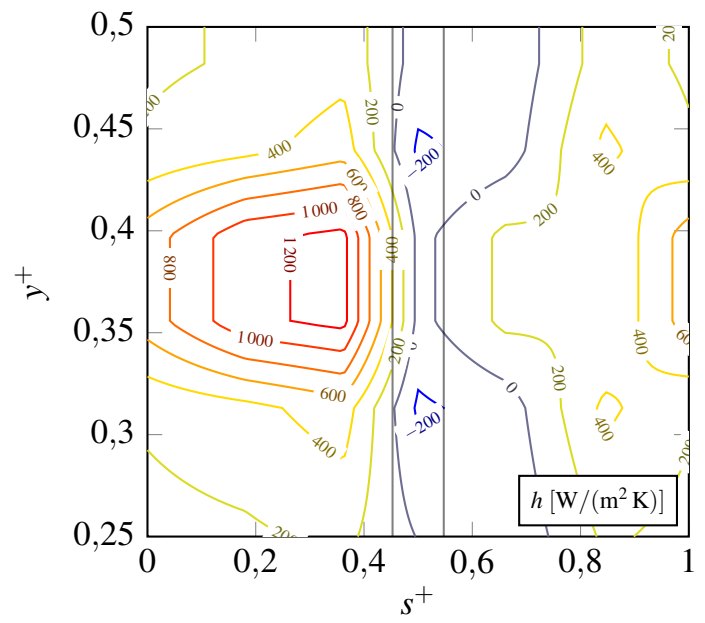

(e) BP41

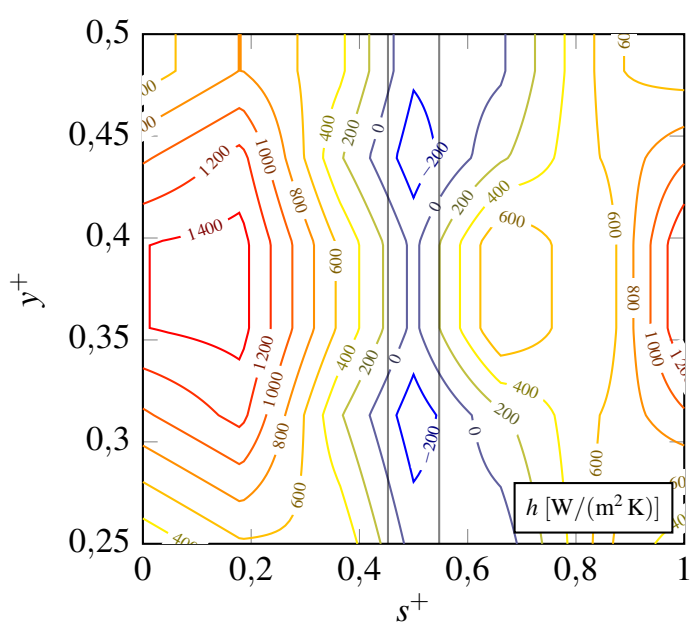

(b) BP38

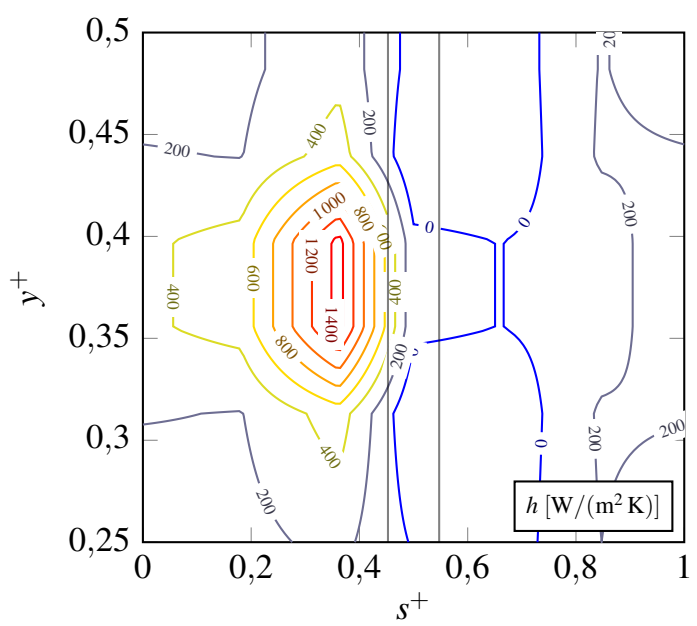

(d) BP40

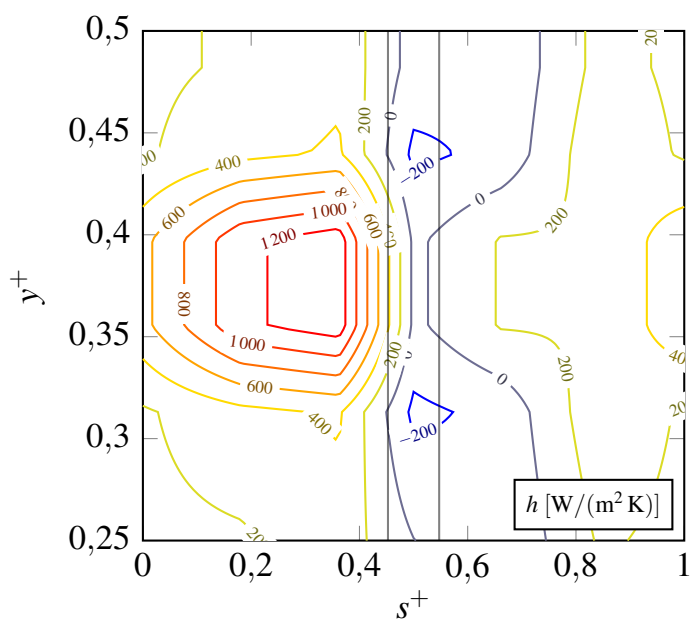

(f) BP42

Abbildung A.7: Ortsaufgelöste Wärmeübergangsmessungen für BP37 bis BP42 


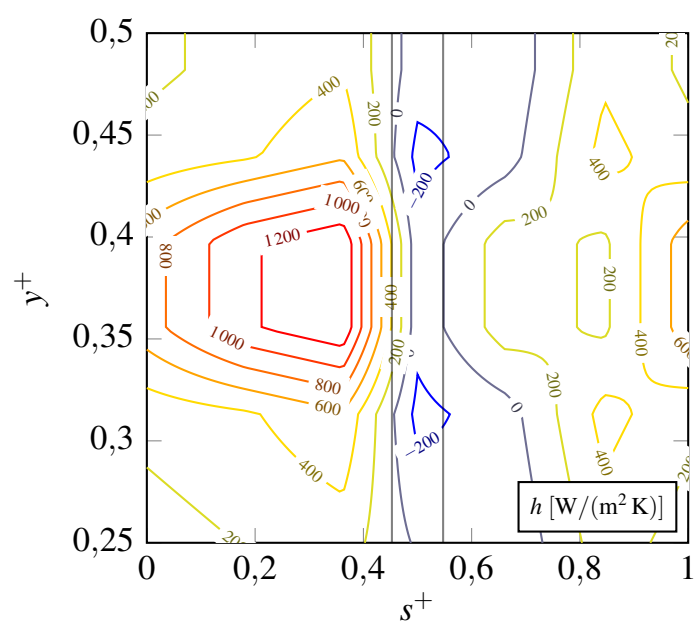

(a) BP43

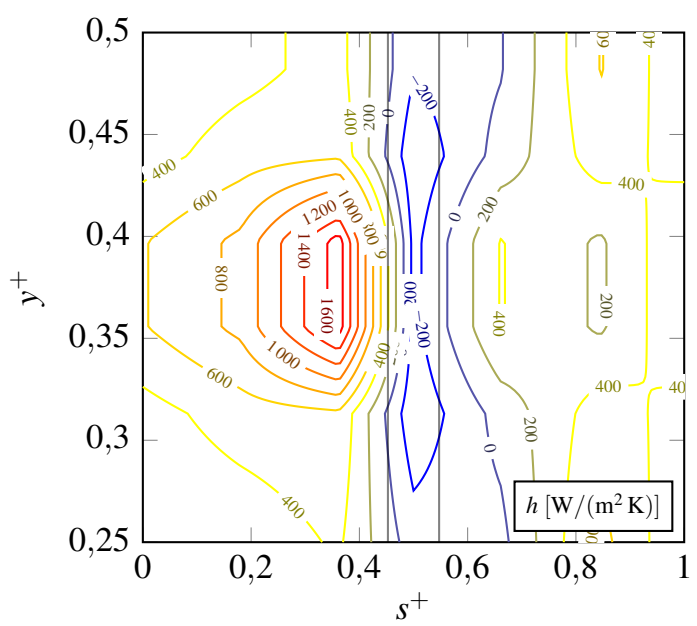

(c) BP45

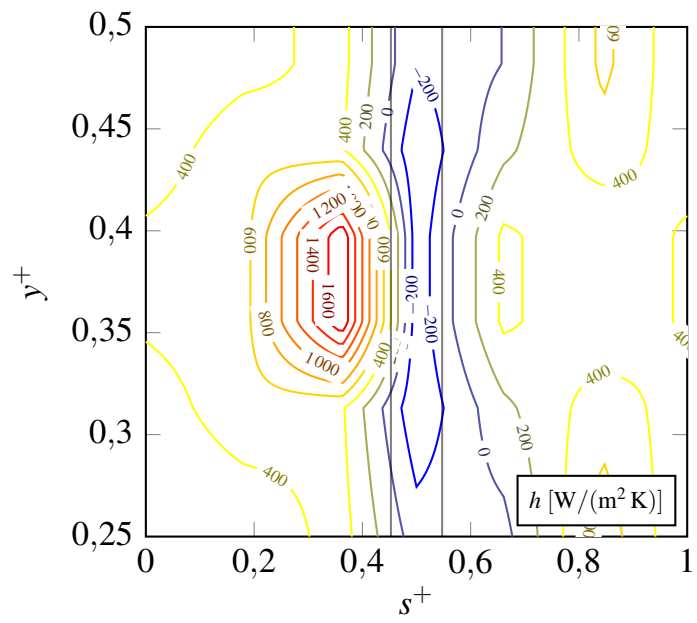

(e) BP47

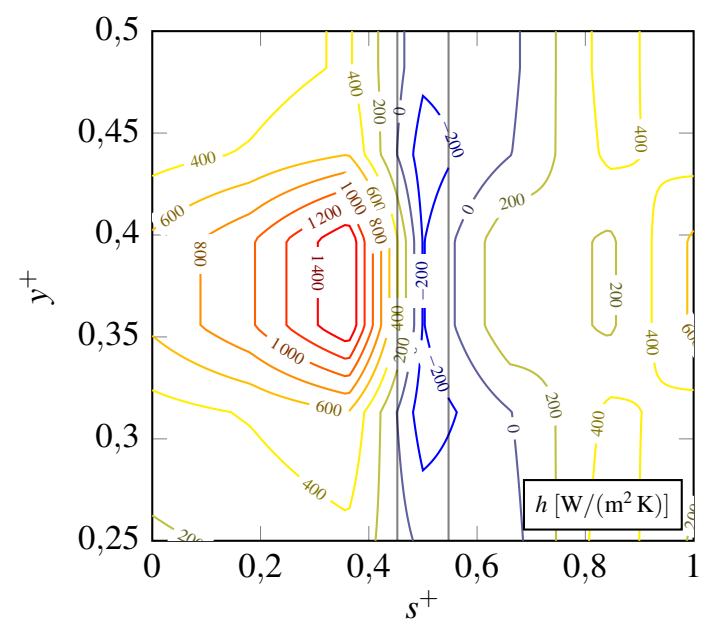

(b) BP44

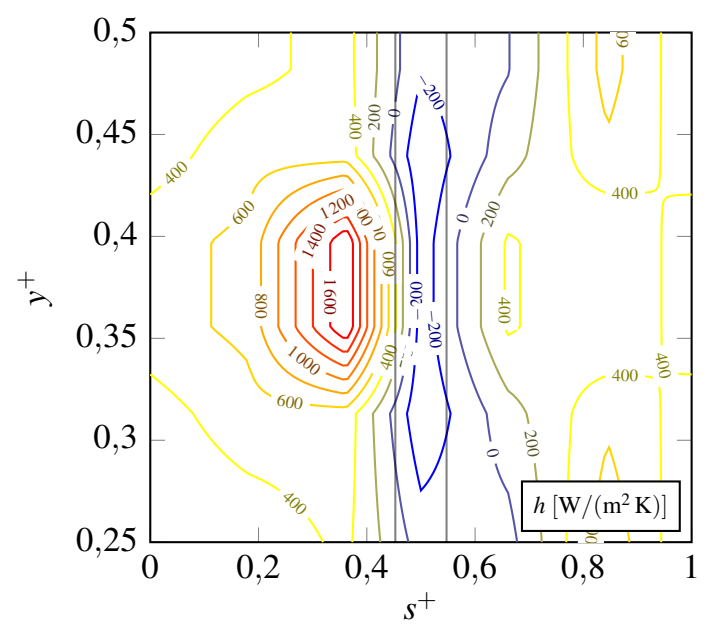

(d) BP46

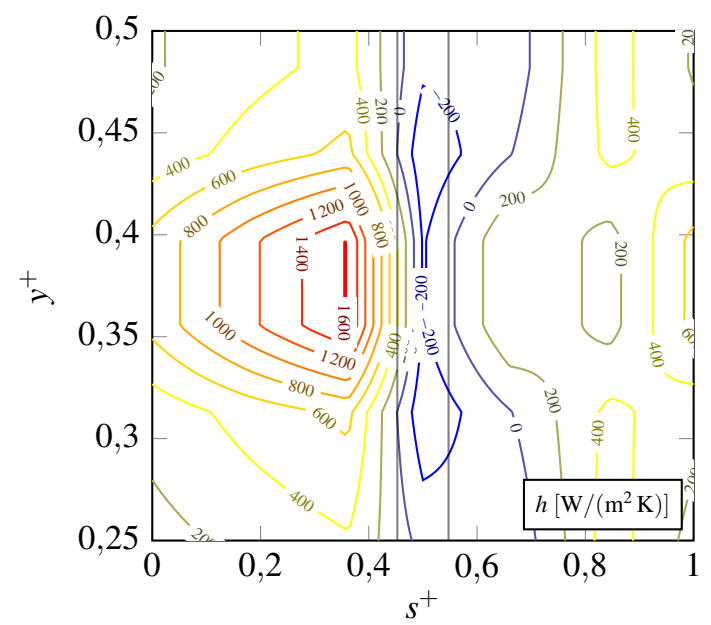

(f) BP48

Abbildung A.8: Ortsaufgelöste Wärmeübergangsmessungen für BP43 bis BP48 


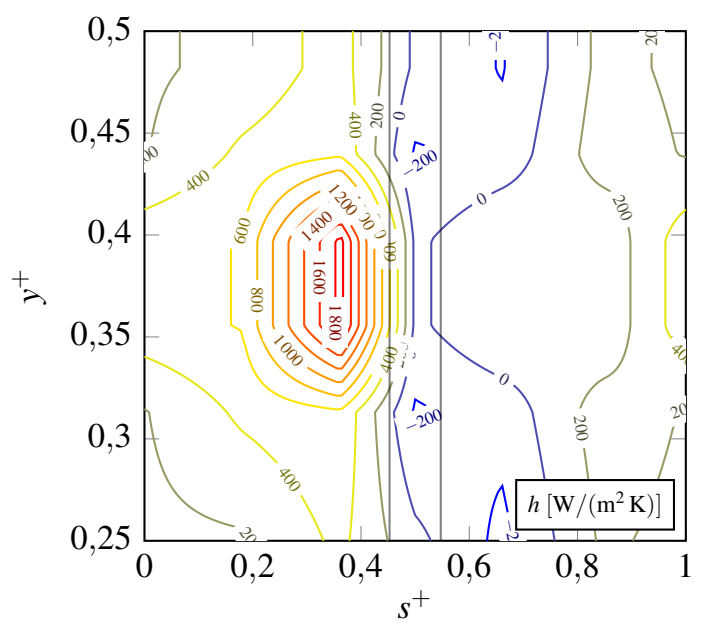

(a) BP49

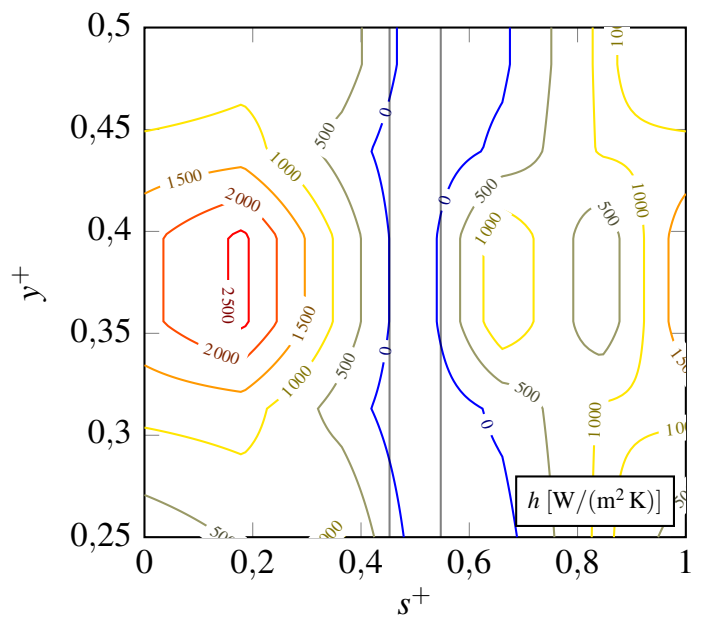

(c) BP51

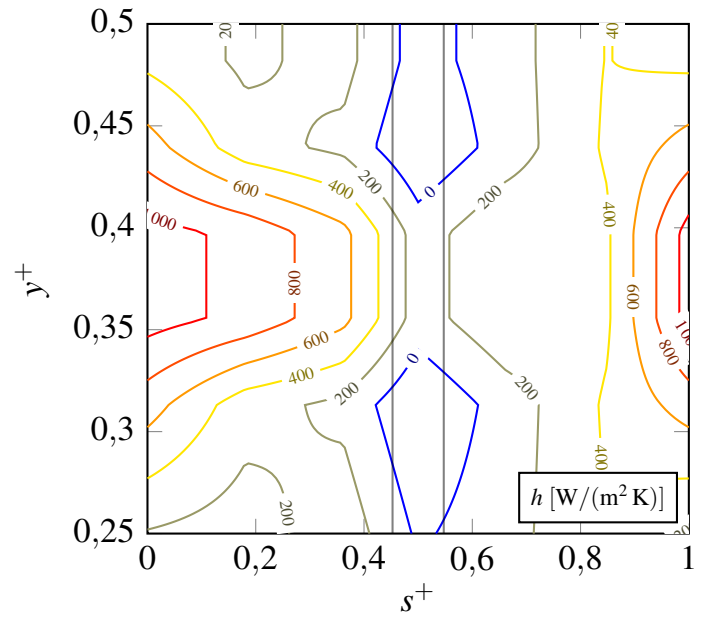

(e) BP53

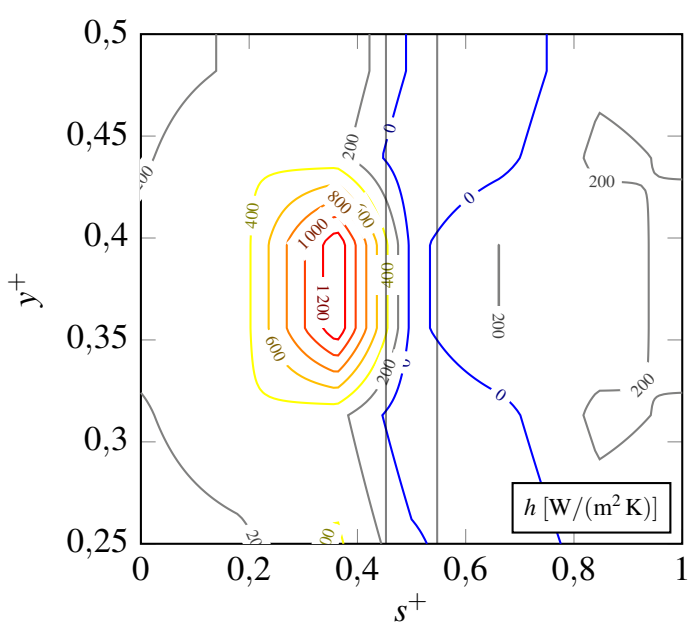

(b) BP50

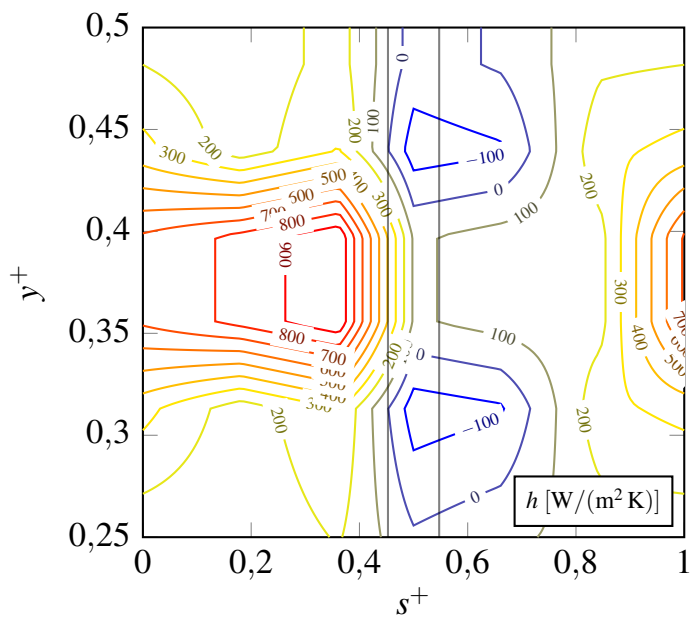

(d) BP52

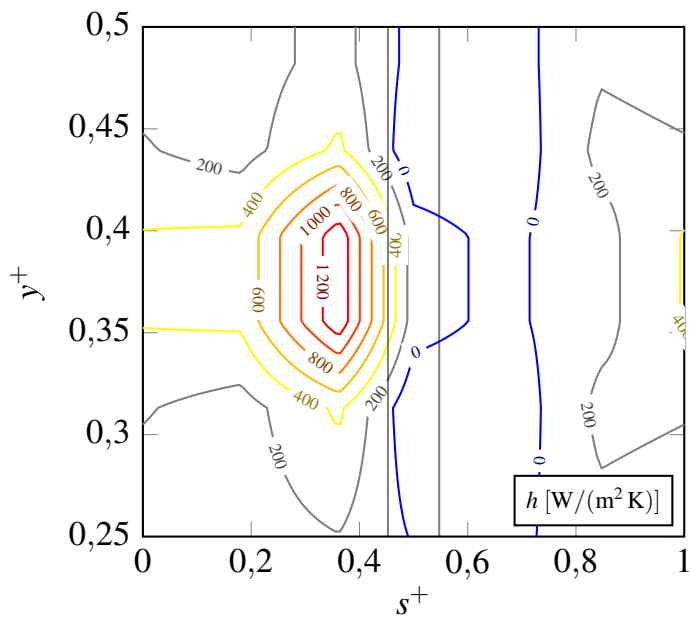

(f) BP54

Abbildung A.9: Ortsaufgelöste Wärmeübergangsmessungen für BP49 bis BP54 


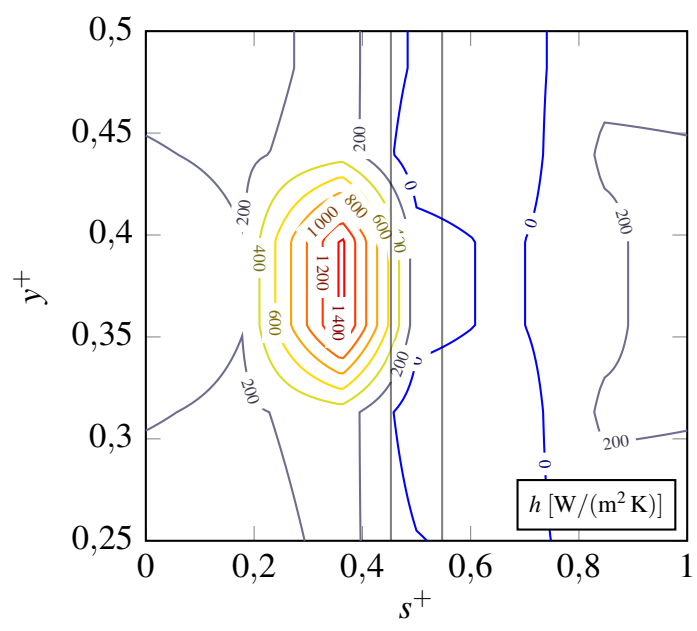

(a) BP55

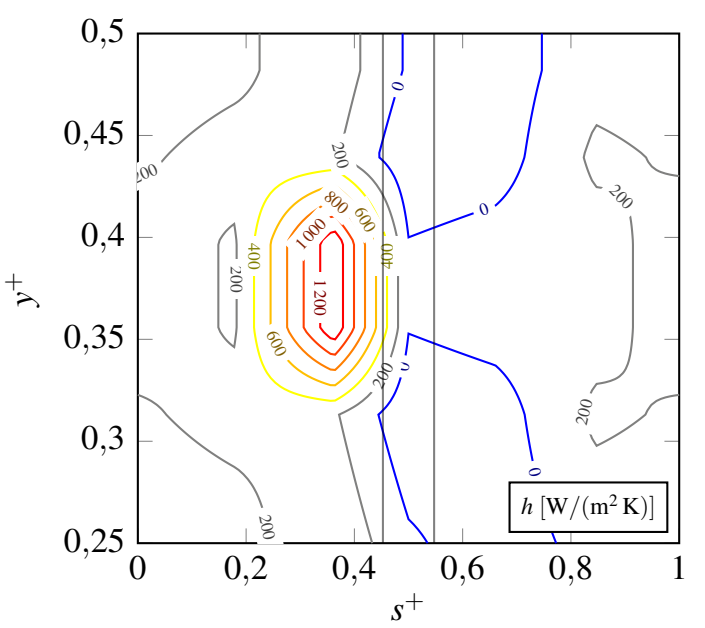

(c) BP57

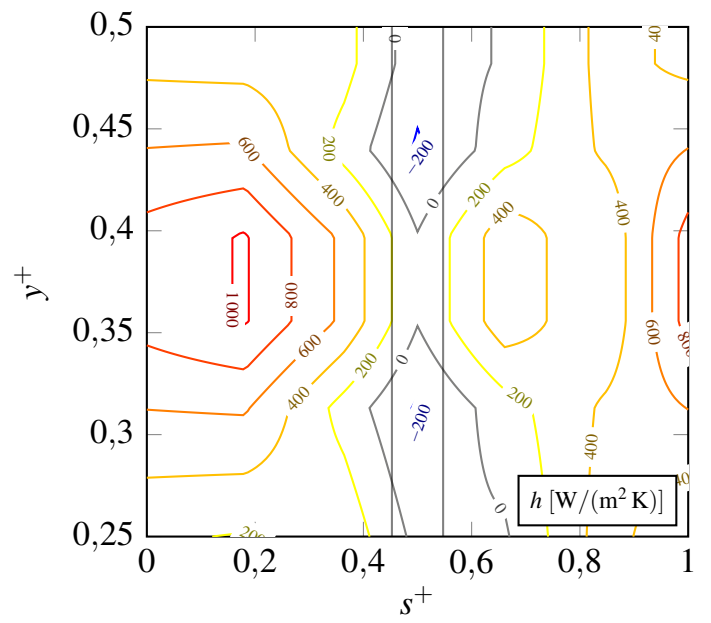

(e) BP59

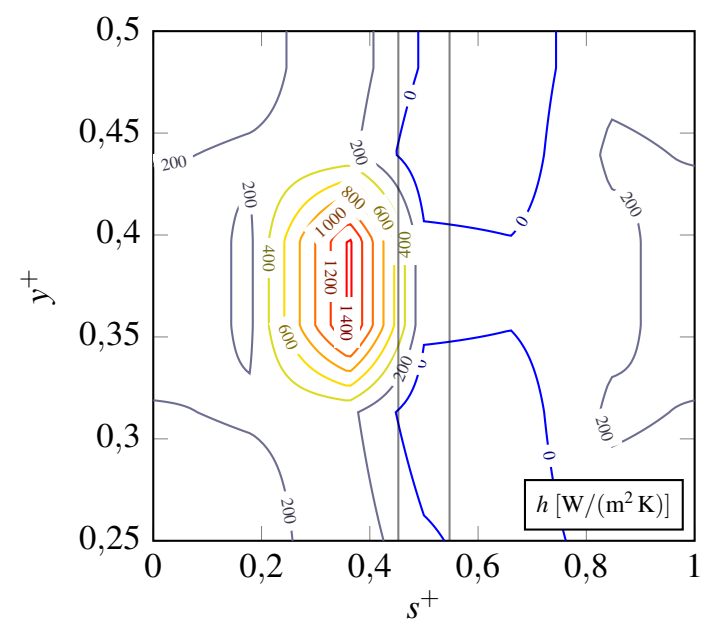

(b) BP56

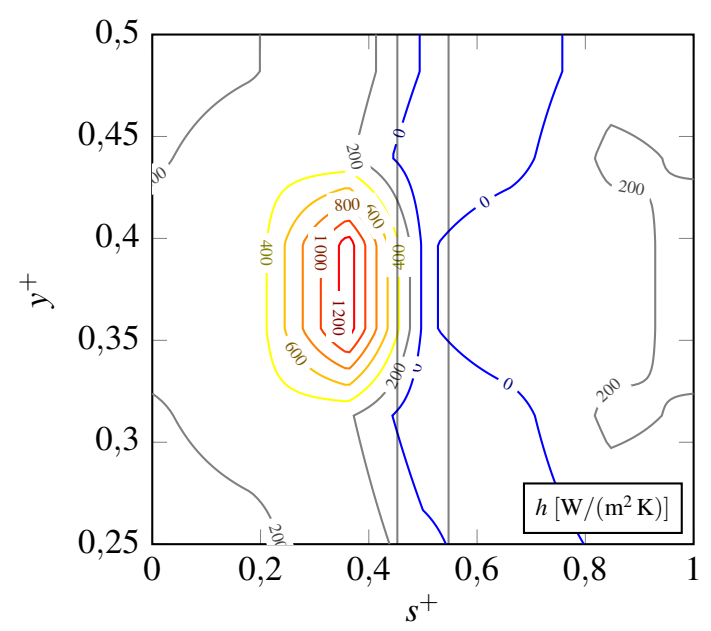

(d) BP58

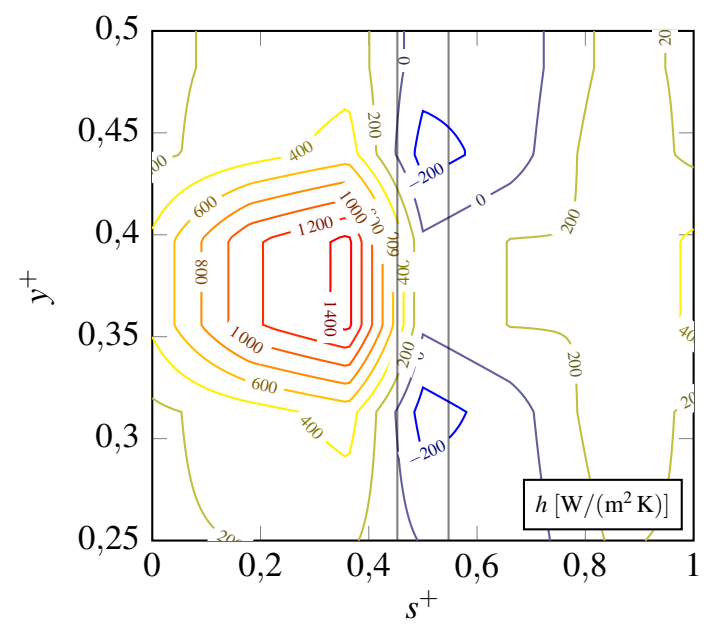

(f) BP60

Abbildung A.10: Ortsaufgelöste Wärmeübergangsmessungen für BP55 bis BP60 


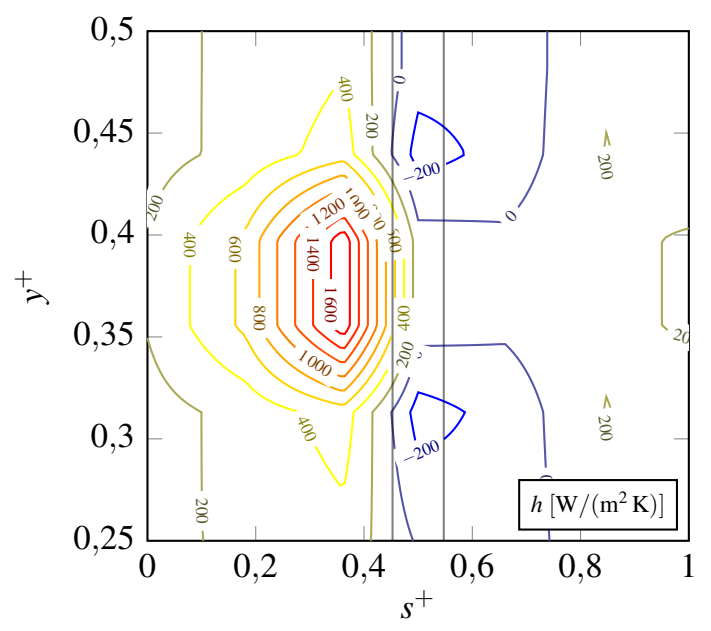

(a) BP61

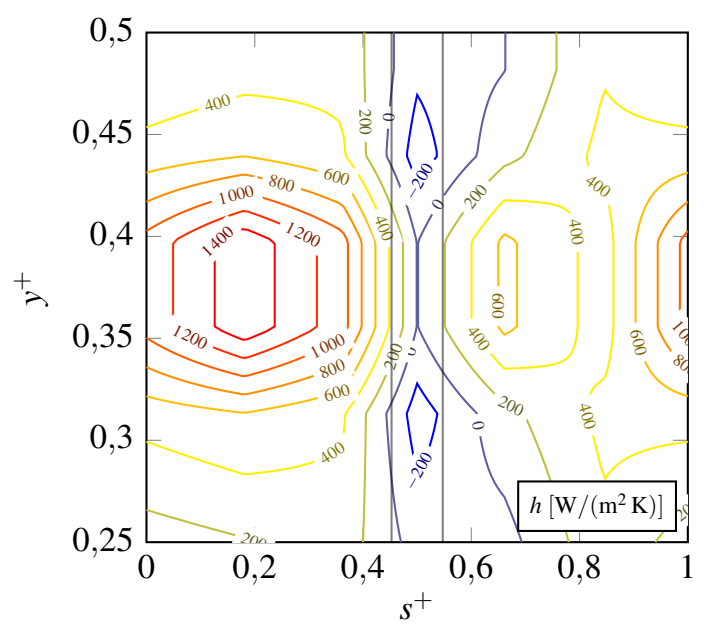

(c) BP63

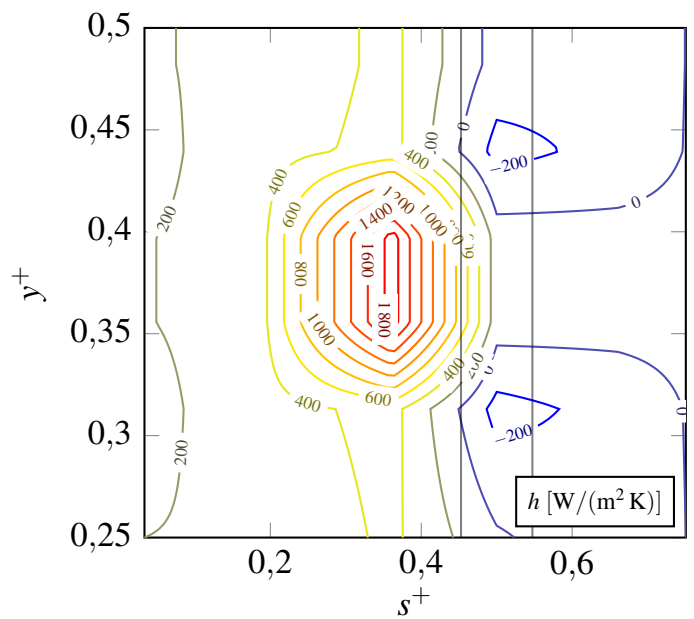

(e) BP65

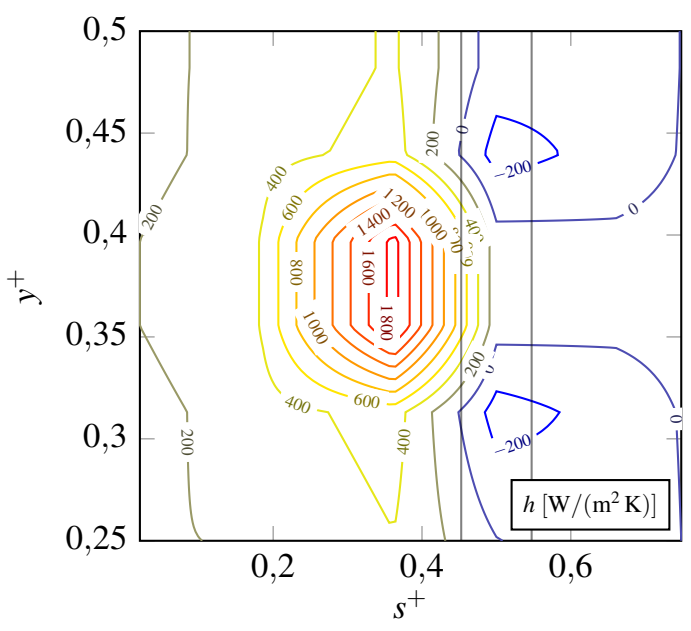

(b) BP62

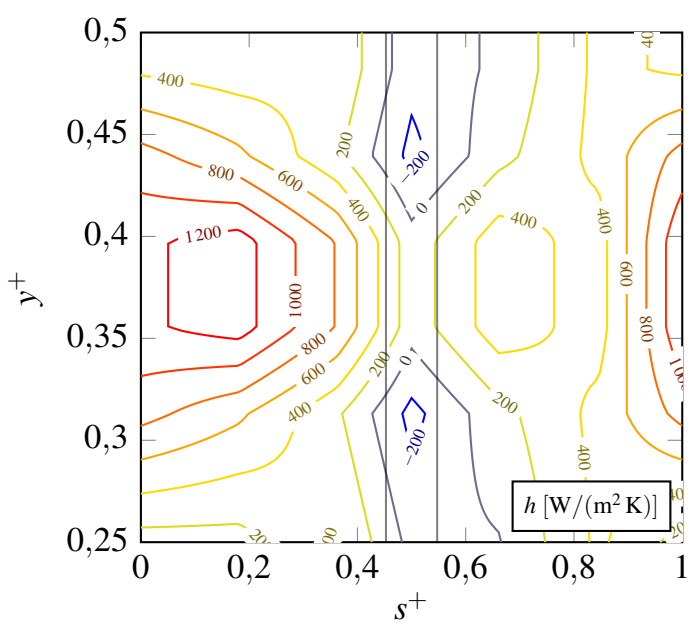

(d) BP64

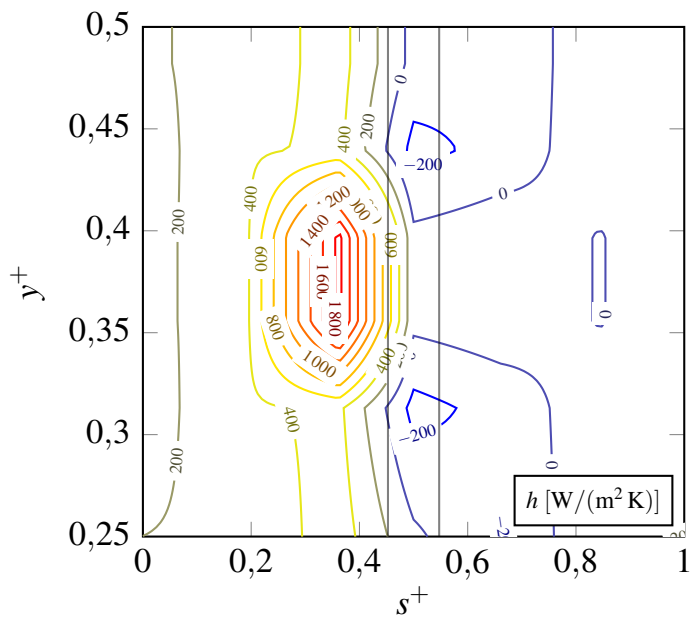

(f) BP66

Abbildung A.11: Ortsaufgelöste Wärmeübergangsmessungen für BP61 bis BP66 


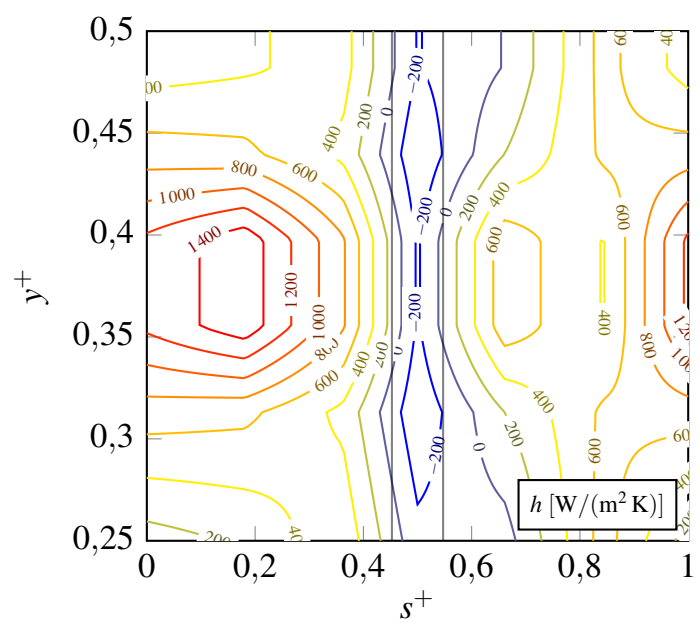

(a) BP67

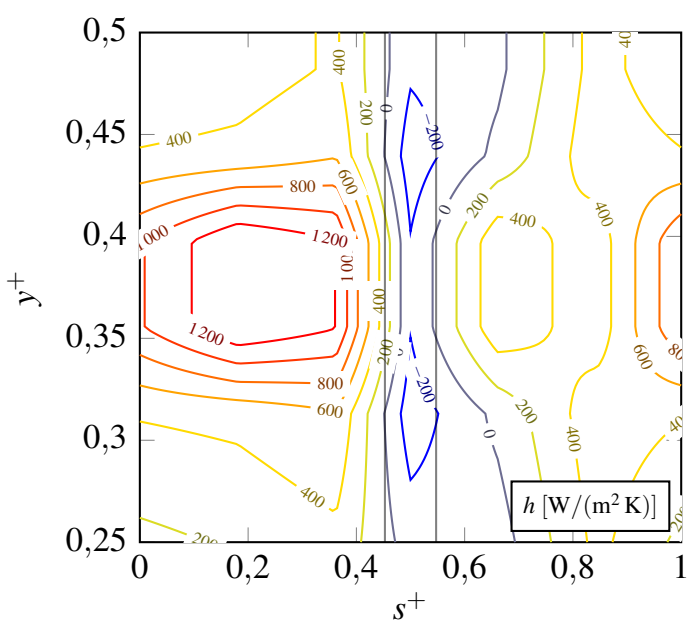

(c) BP69

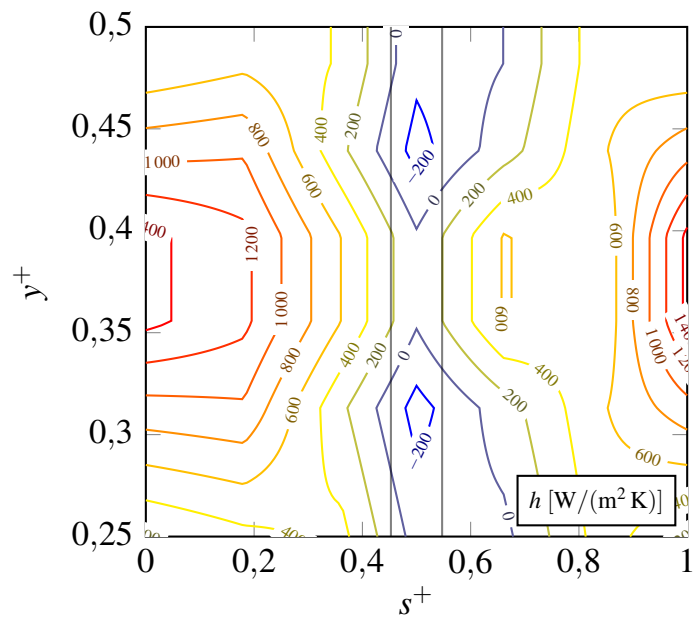

(e) BP71

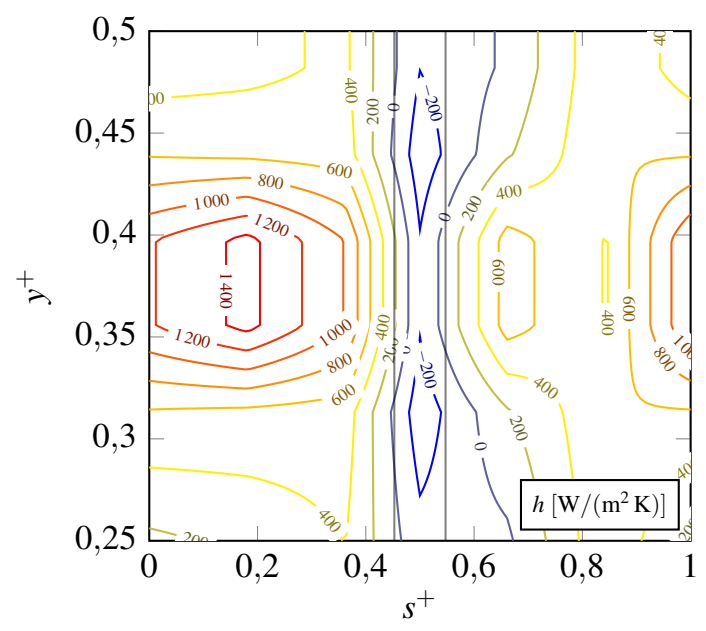

(b) BP68

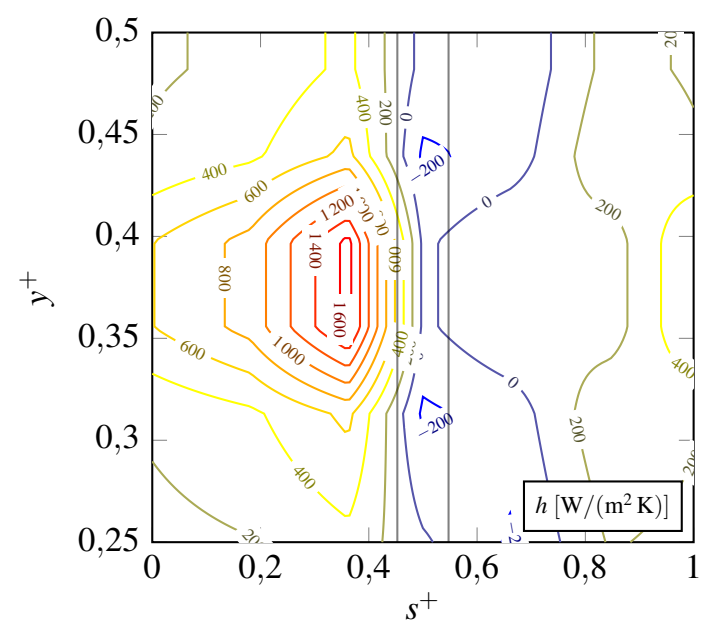

(d) BP70

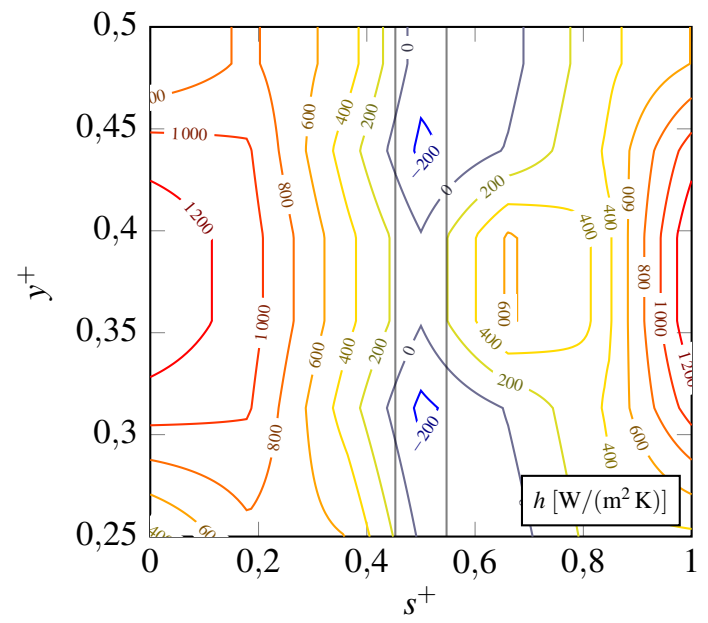

(f) BP72

Abbildung A.12: Ortsaufgelöste Wärmeübergangsmessungen für BP67 bis BP72 


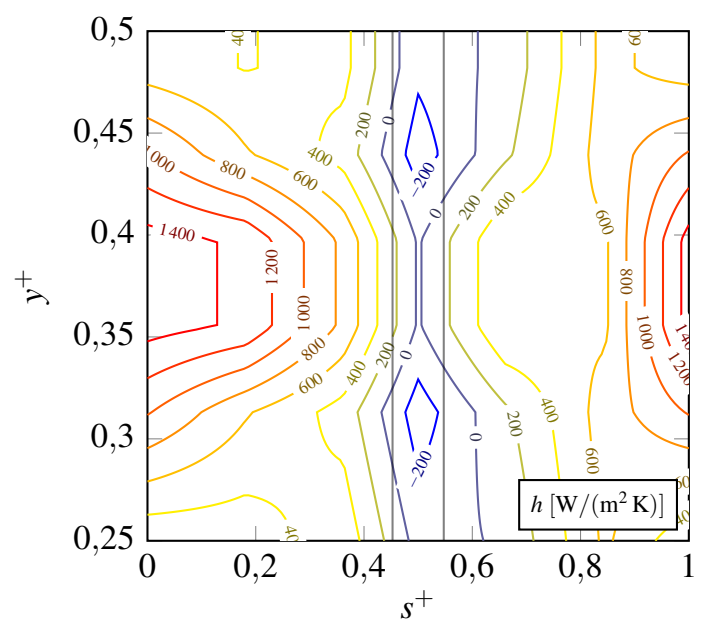

(a) BP73

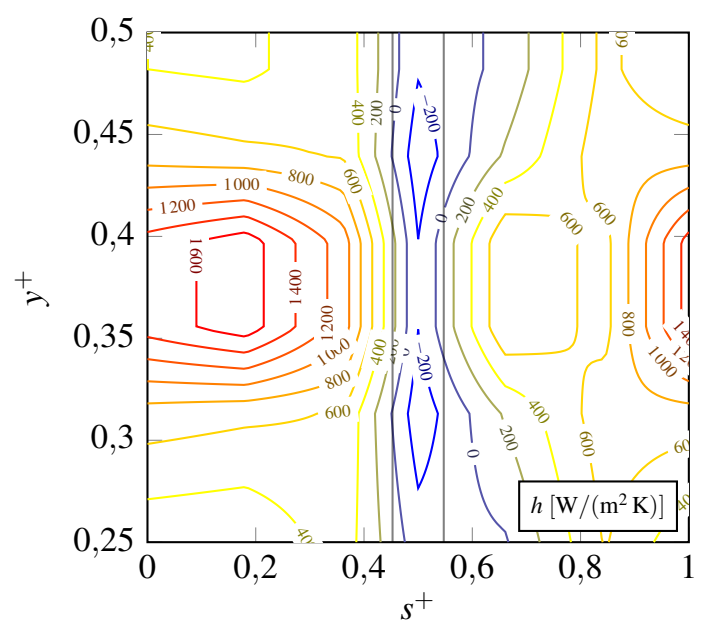

(c) BP75

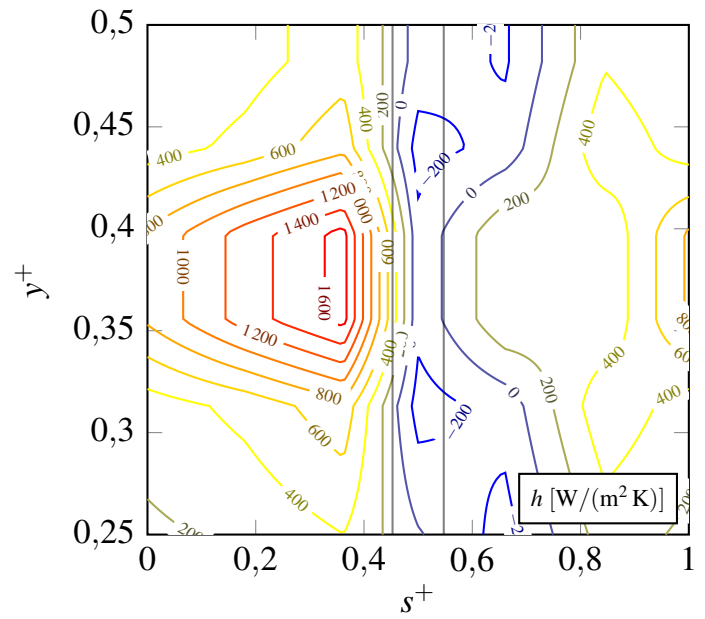

(e) BP77

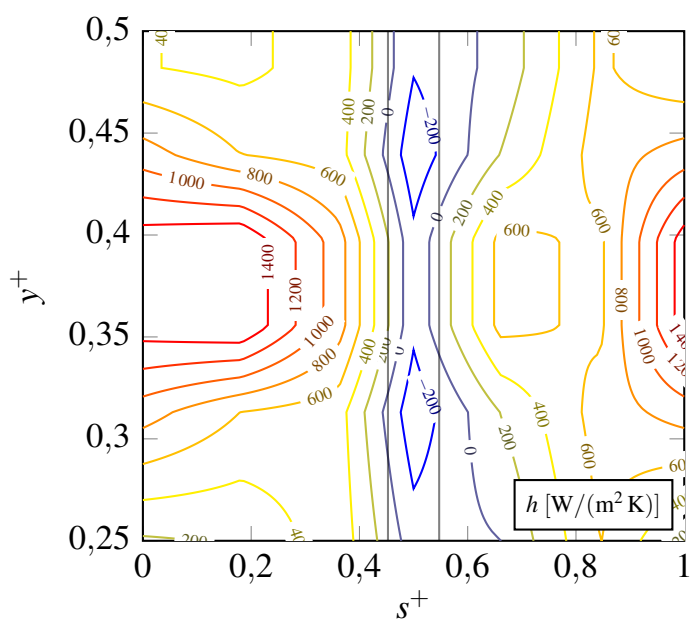

(b) BP74

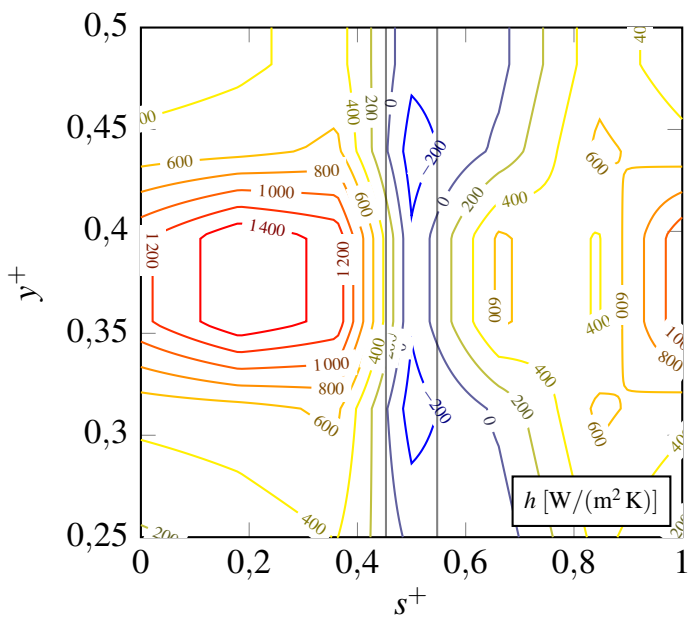

(d) BP76

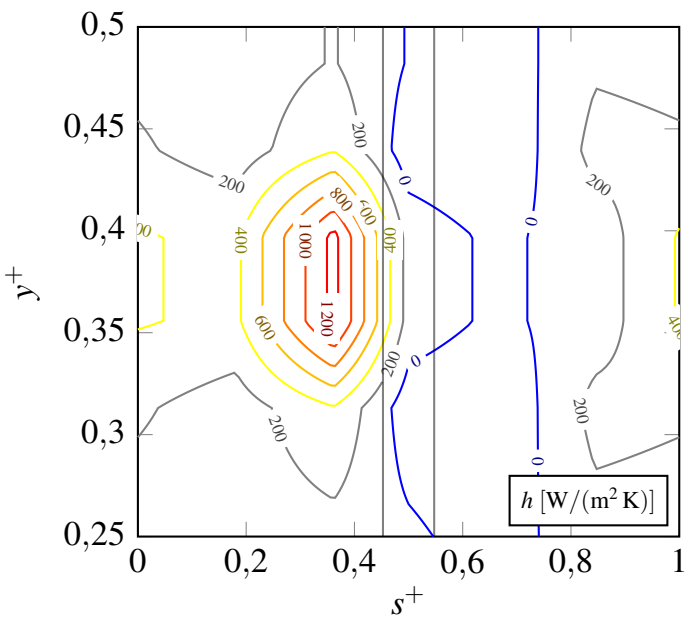

(f) BP78

Abbildung A.13: Ortsaufgelöste Wärmeübergangsmessungen für BP73 bis BP78 


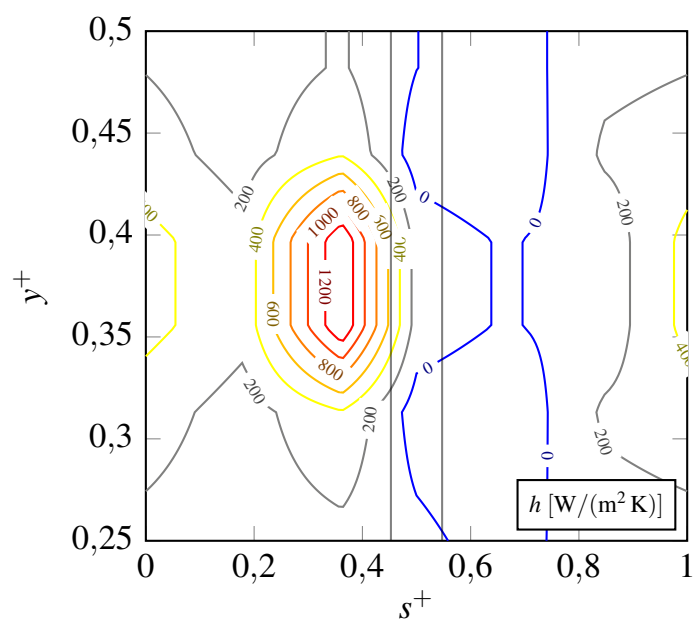

(a) BP79

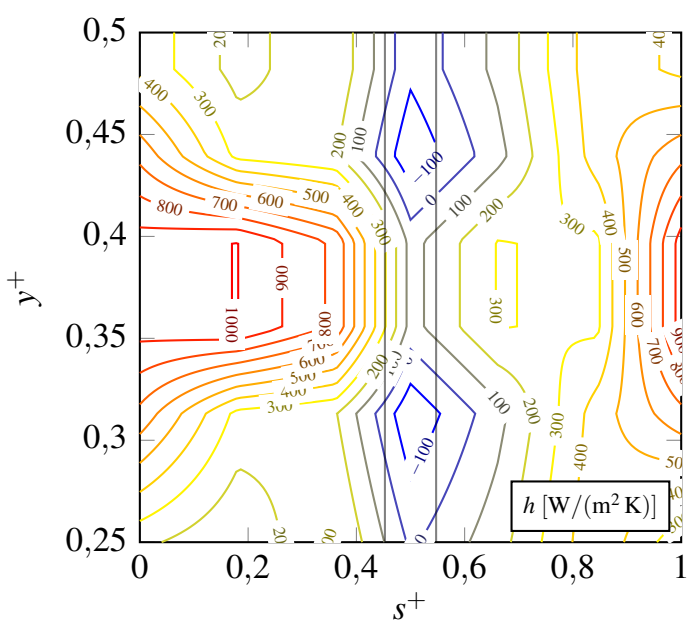

(c) BP81

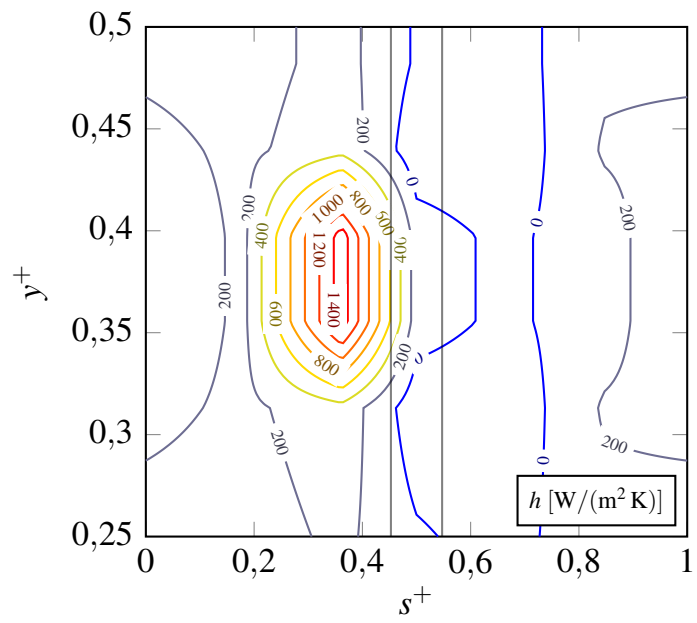

(e) BP83

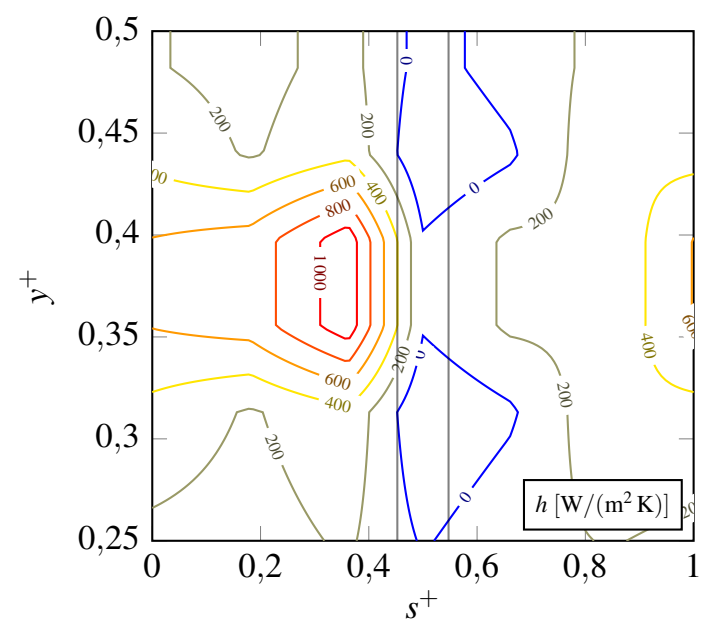

(b) BP80

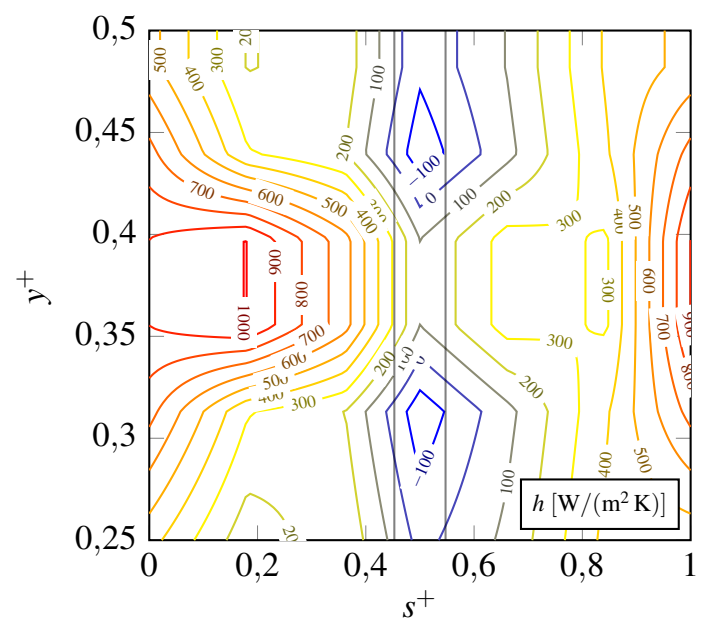

(d) BP82

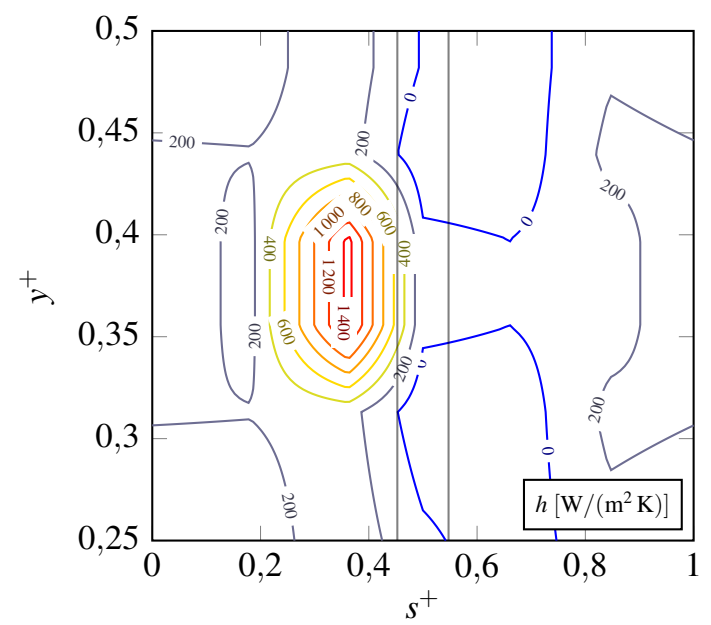

(f) BP84

Abbildung A.14: Ortsaufgelöste Wärmeübergangsmessungen für BP79 bis BP84 


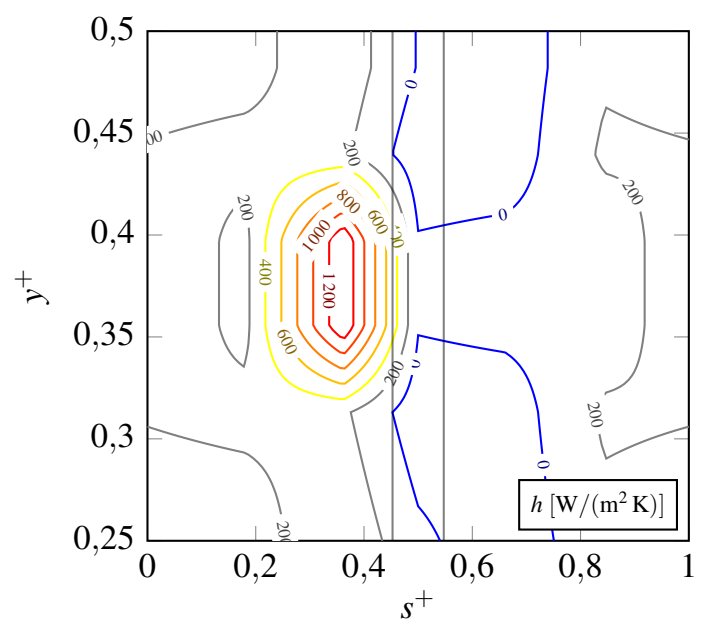

(a) BP85

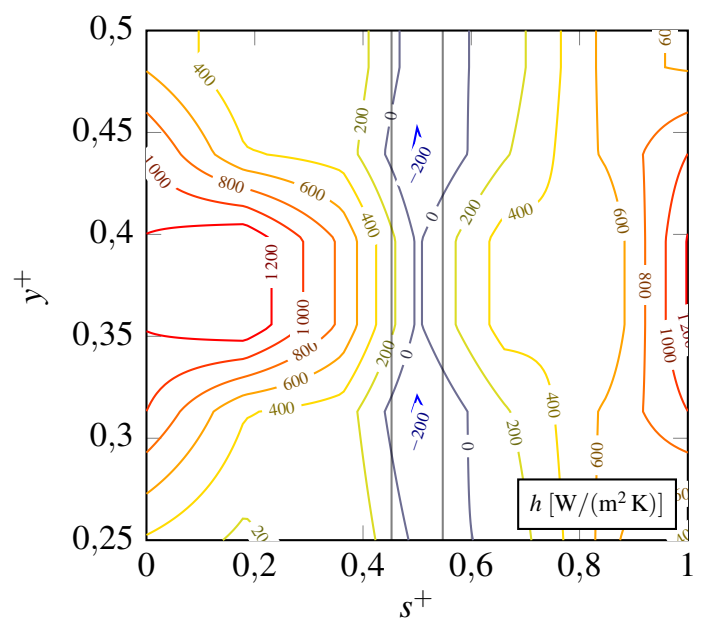

(c) BP87

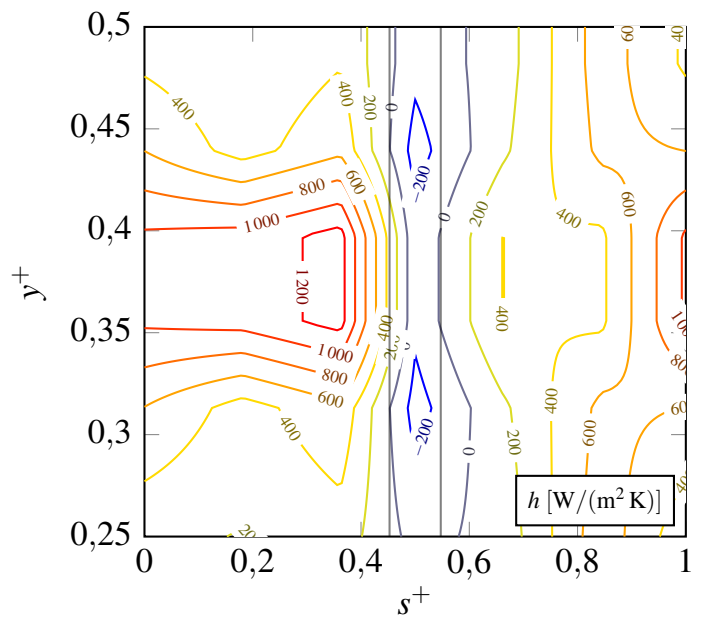

(e) BP89

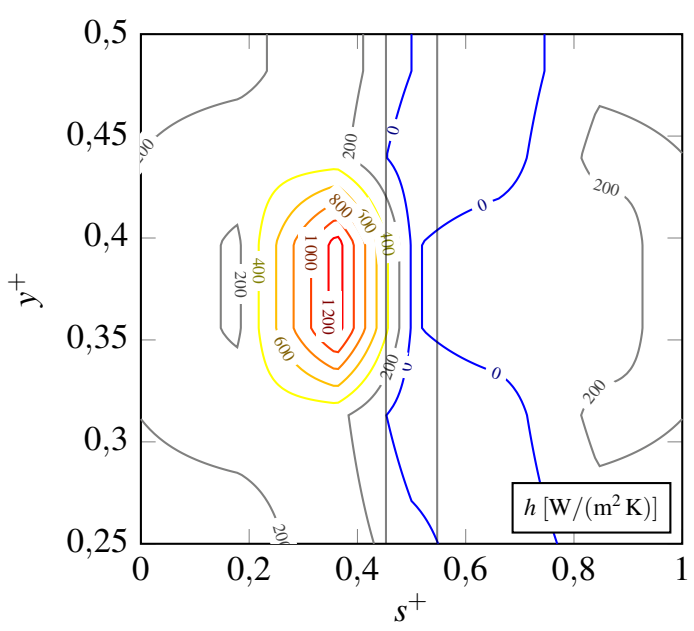

(b) BP86

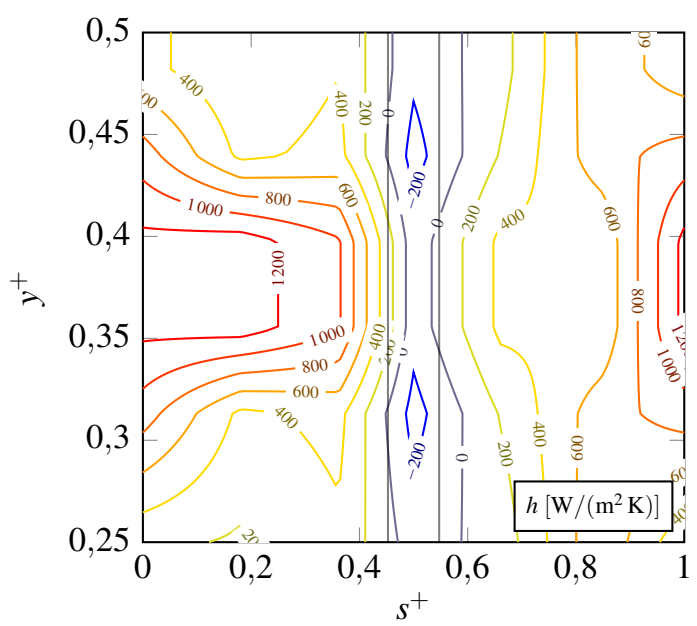

(d) BP88

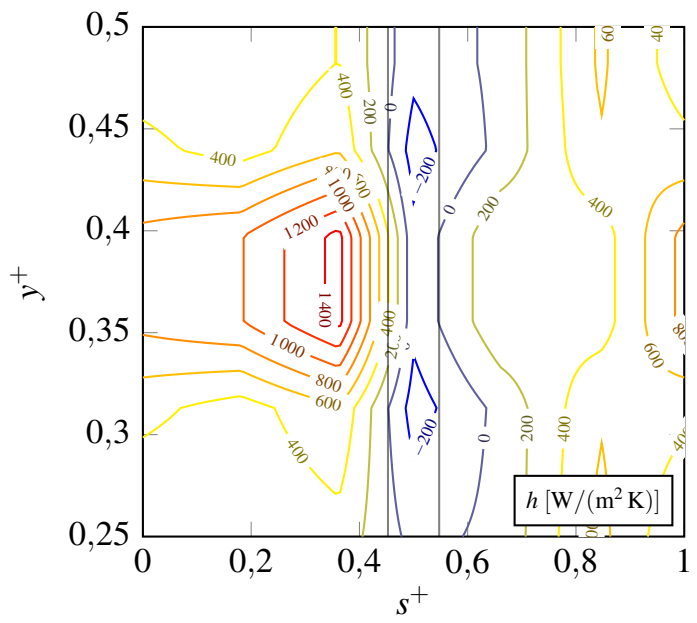

(f) BP90

Abbildung A.15: Ortsaufgelöste Wärmeübergangsmessungen für BP85 bis BP90 


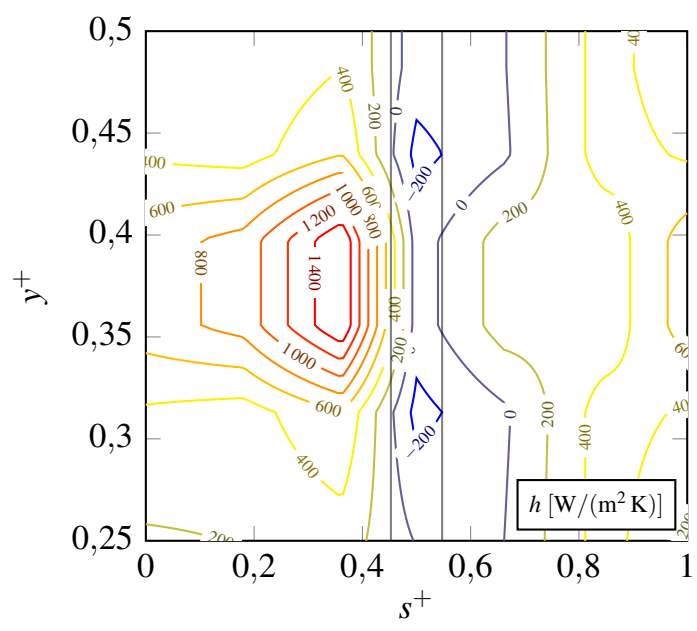

(a) BP91

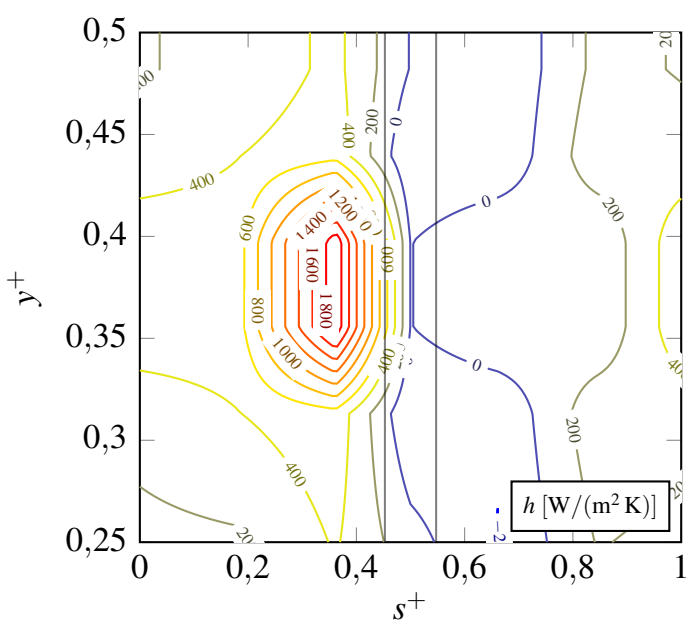

(c) BP93

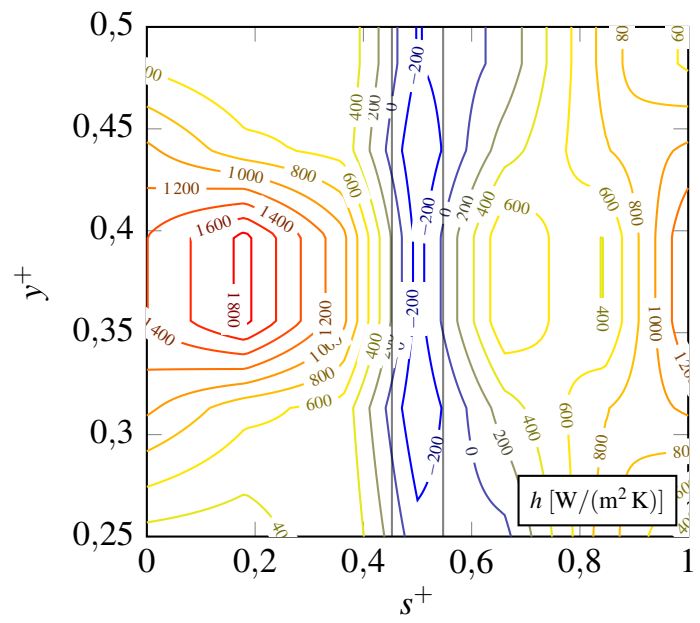

(e) BP95

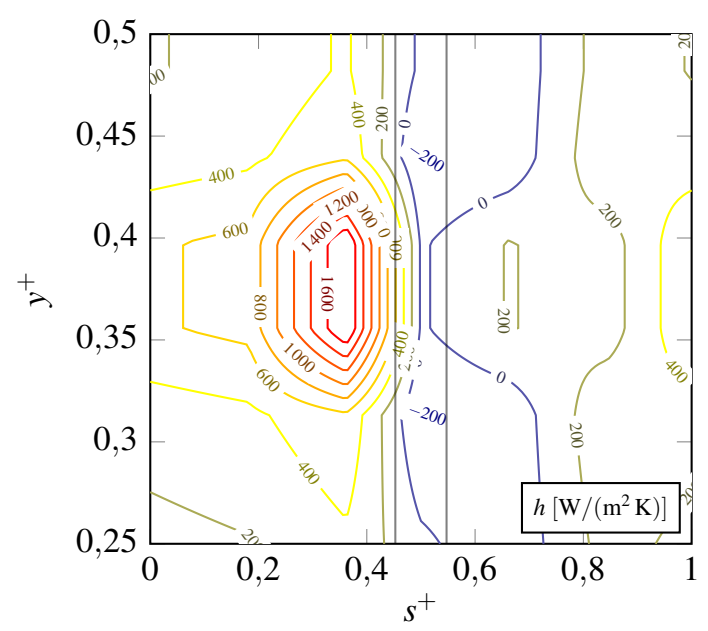

(b) BP92

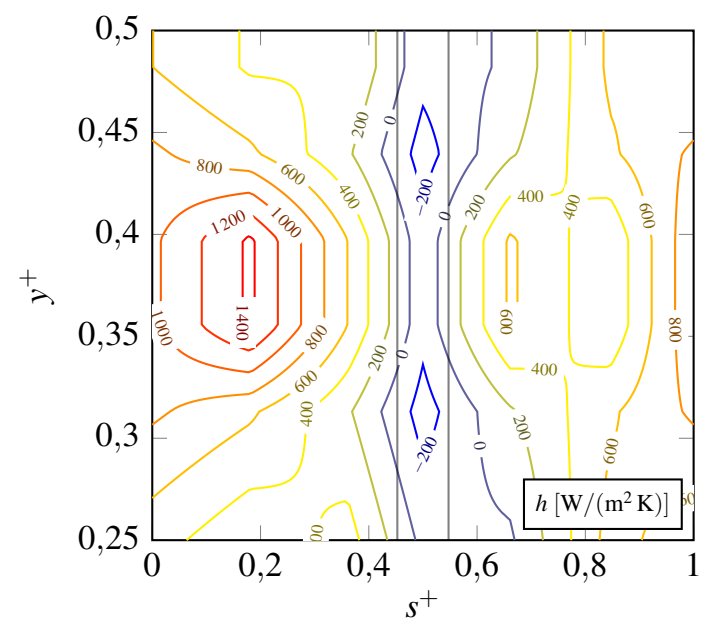

(d) BP94

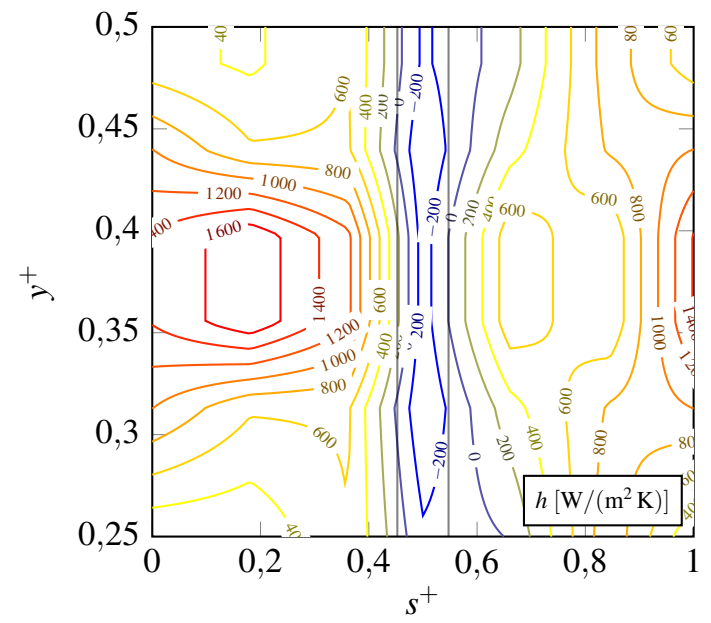

(f) BP96

Abbildung A.16: Ortsaufgelöste Wärmeübergangsmessungen für BP91 bis BP96 


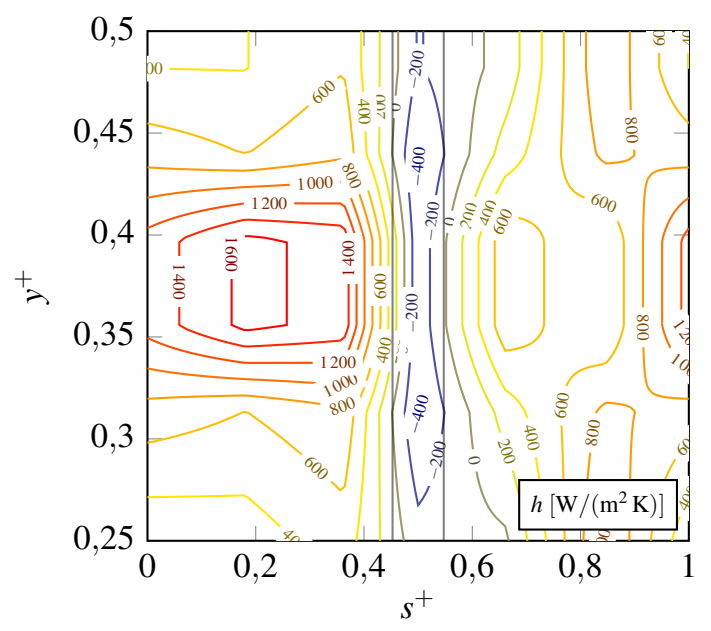

(a) BP97

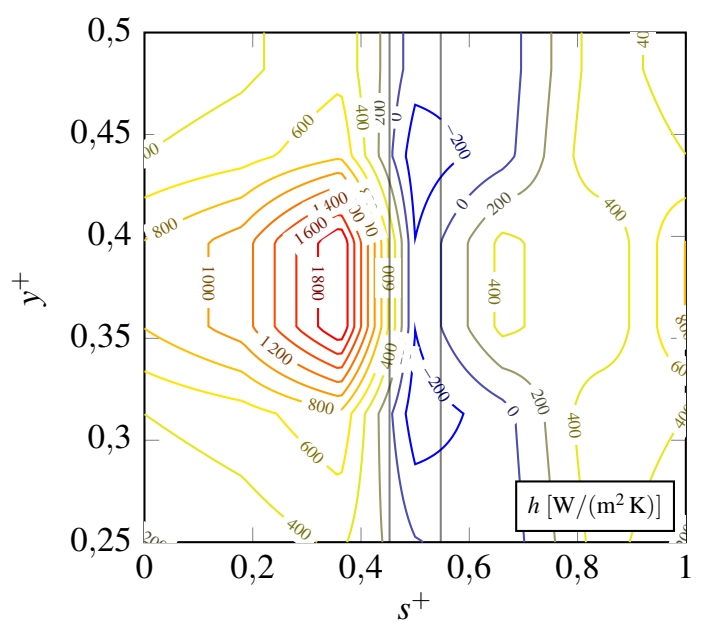

(c) BP99

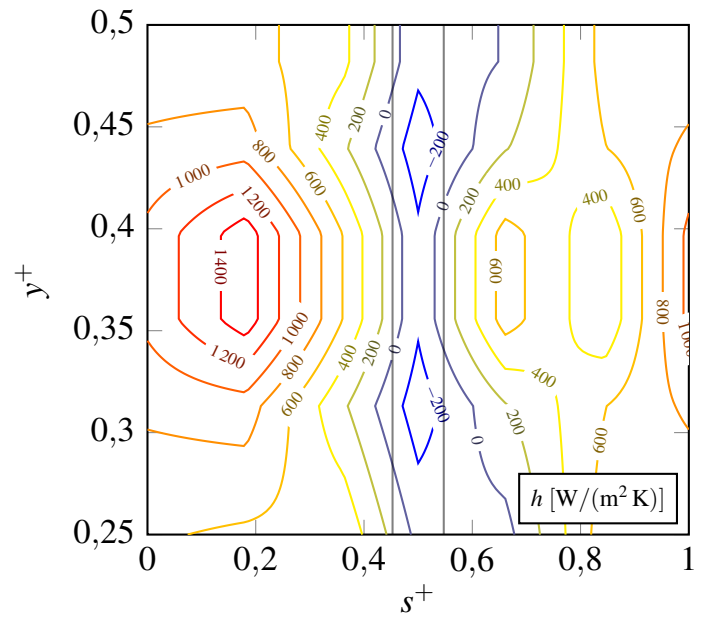

(e) BP101

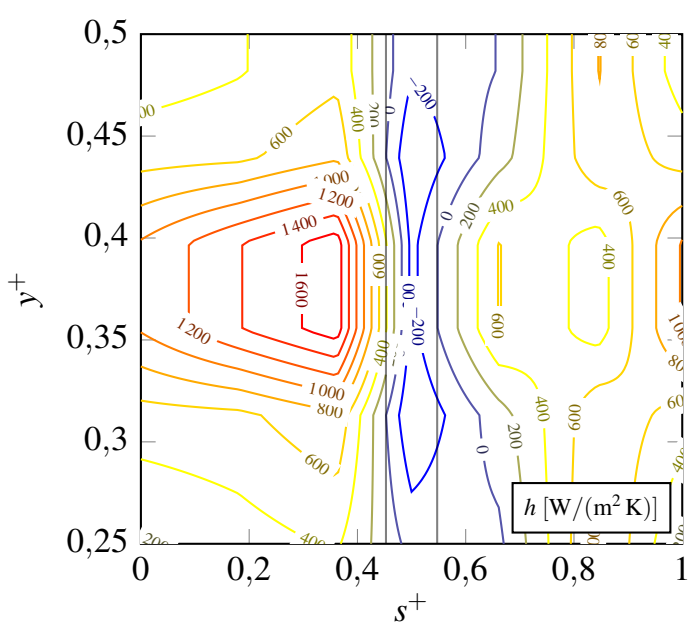

(b) BP98

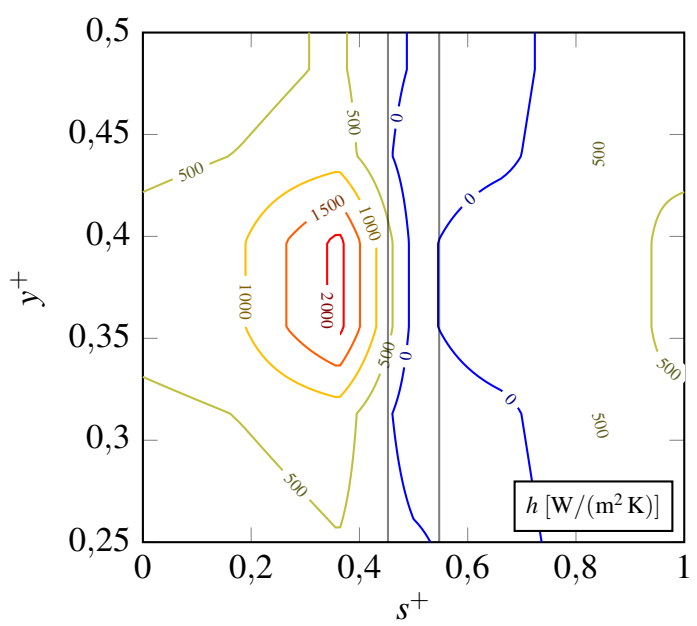

(d) BP100

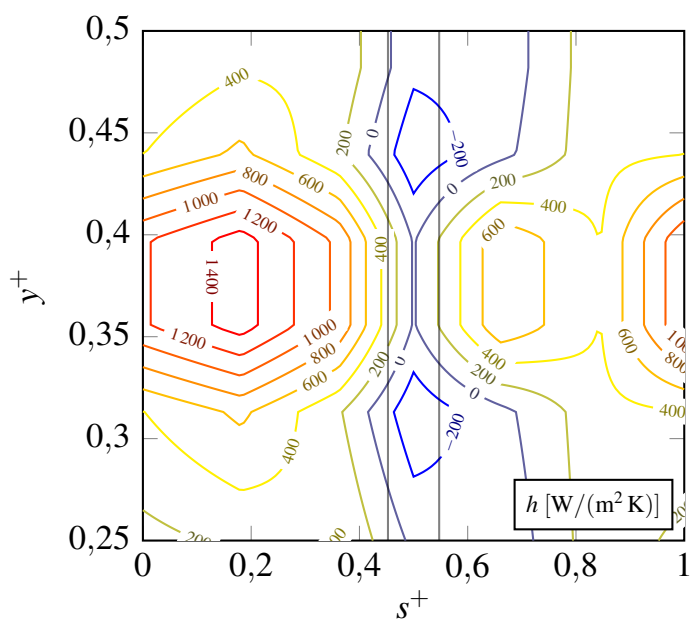

(f) BP102

Abbildung A.17: Ortsaufgelöste Wärmeübergangsmessungen für BP97 bis BP102 


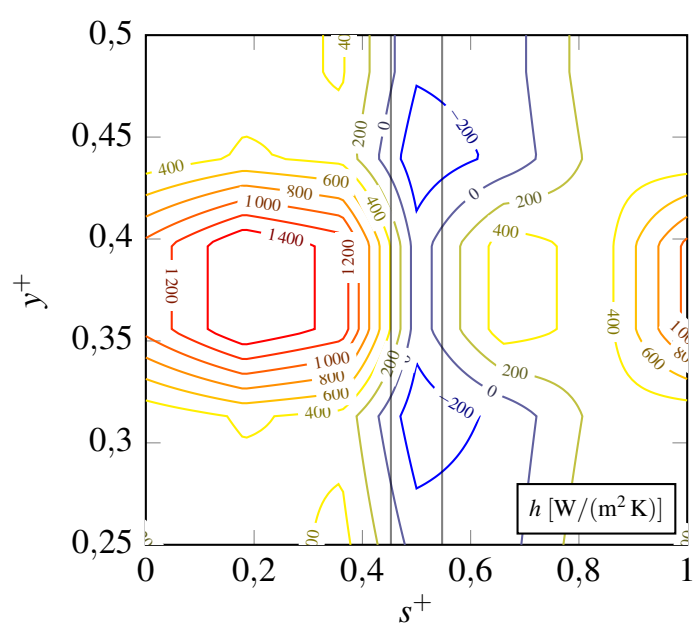

(a) BP103

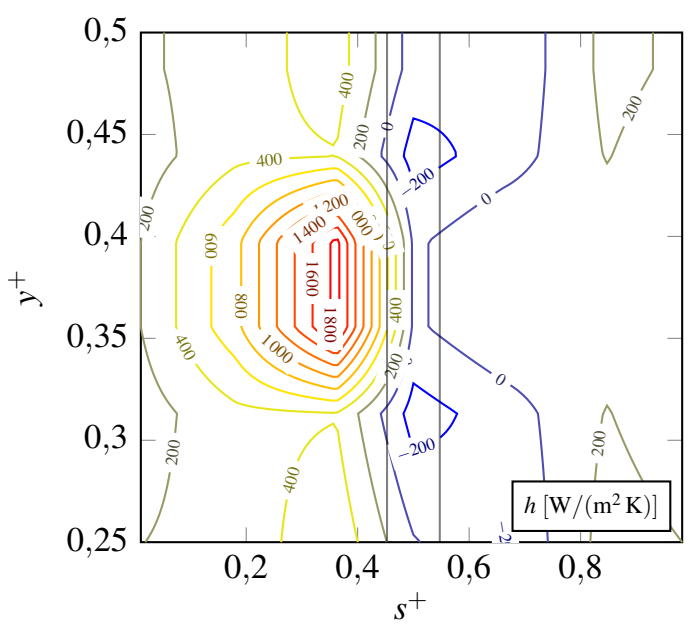

(c) BP105

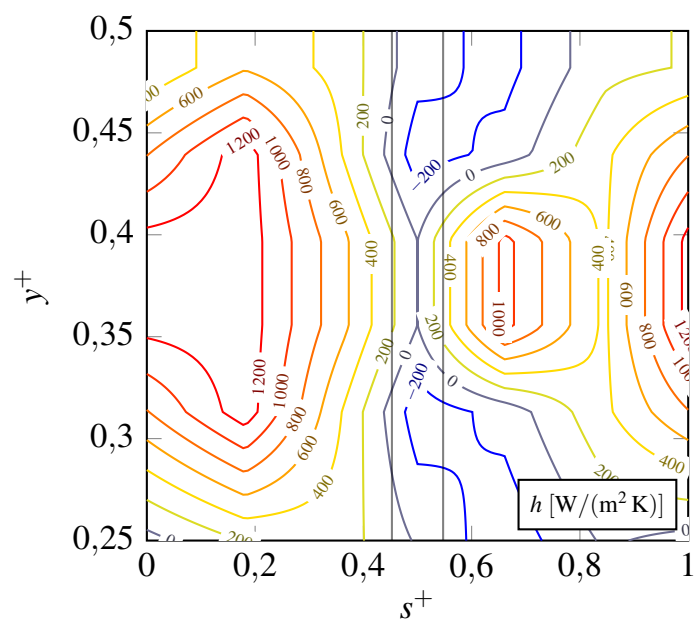

(e) BP107

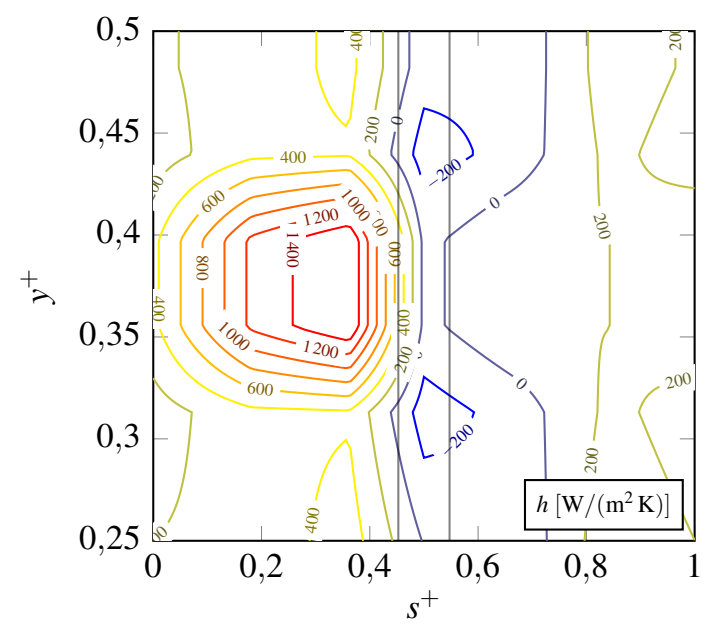

(b) BP104

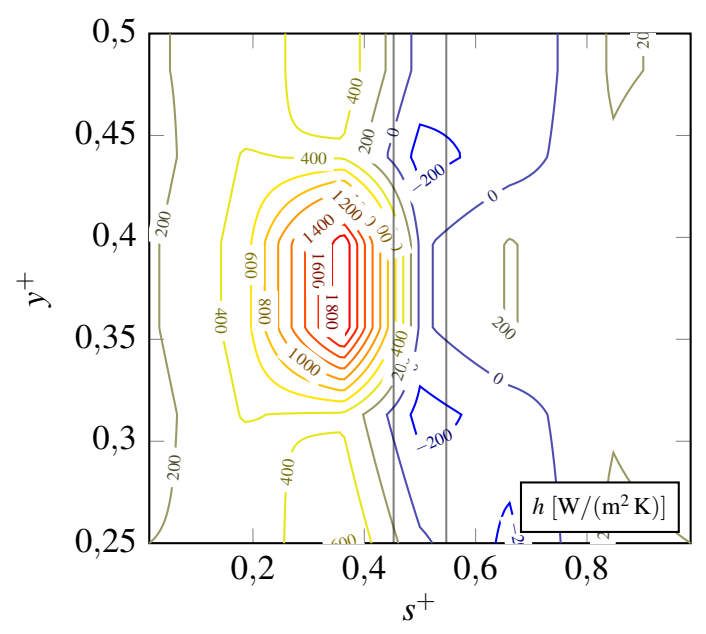

(d) BP106

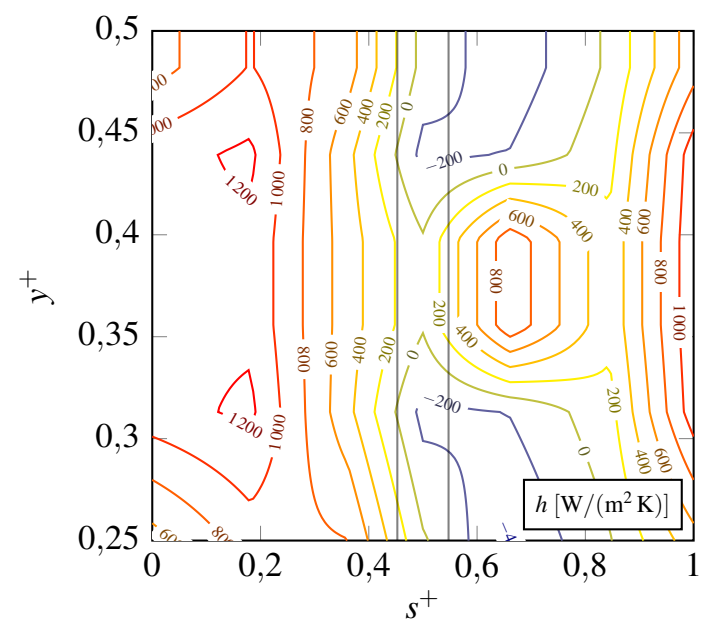

(f) BP108

Abbildung A.18: Ortsaufgelöste Wärmeübergangsmessungen für BP103 bis BP108 


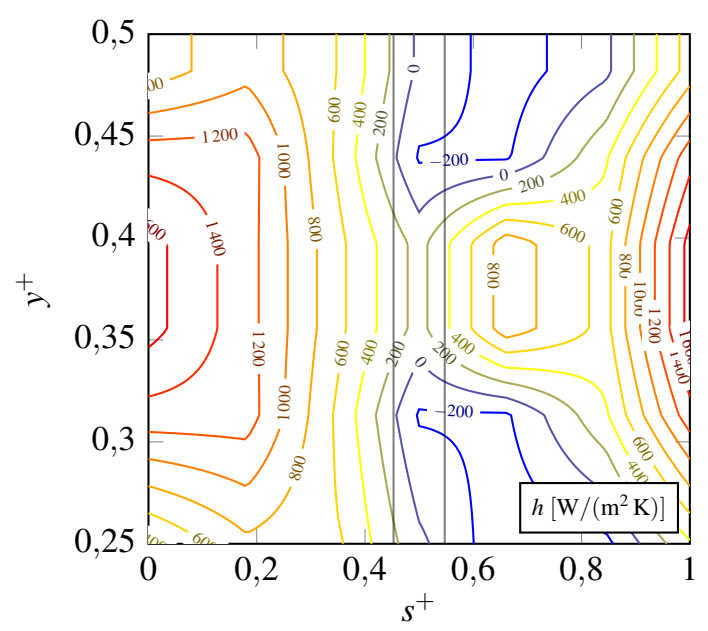

(a) BP109

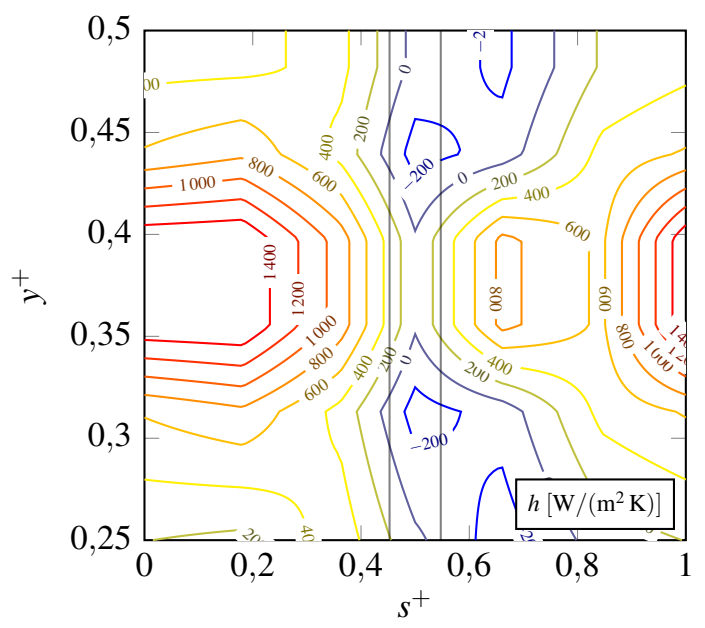

(c) BP111

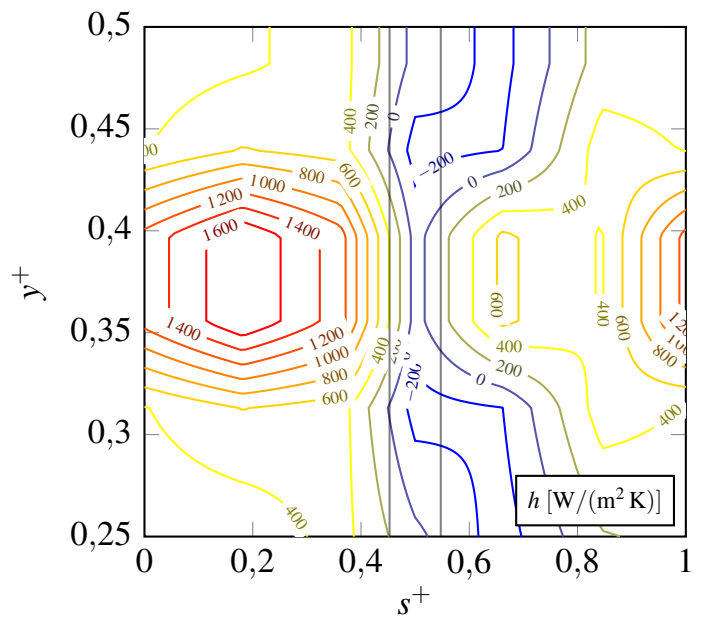

(e) BP113

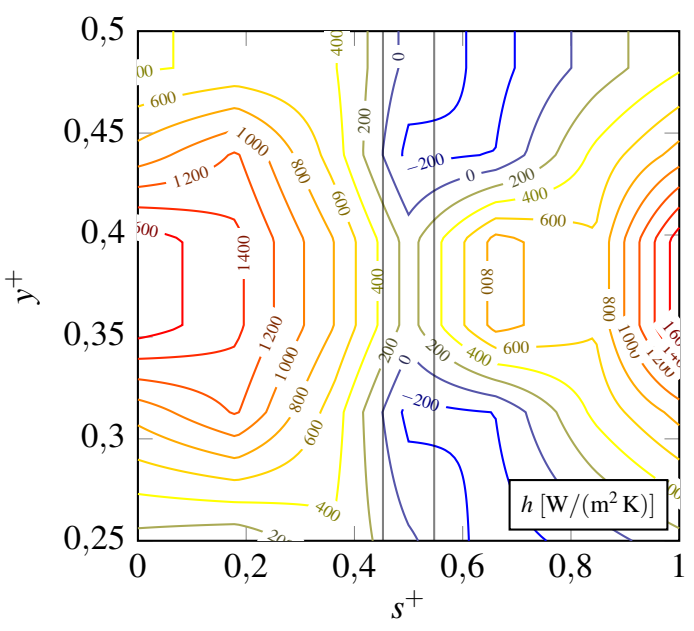

(b) BP110

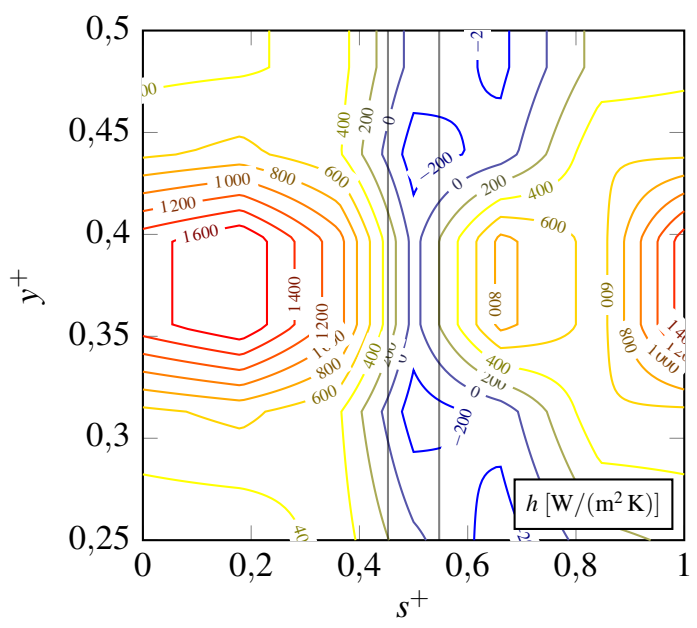

(d) BP112

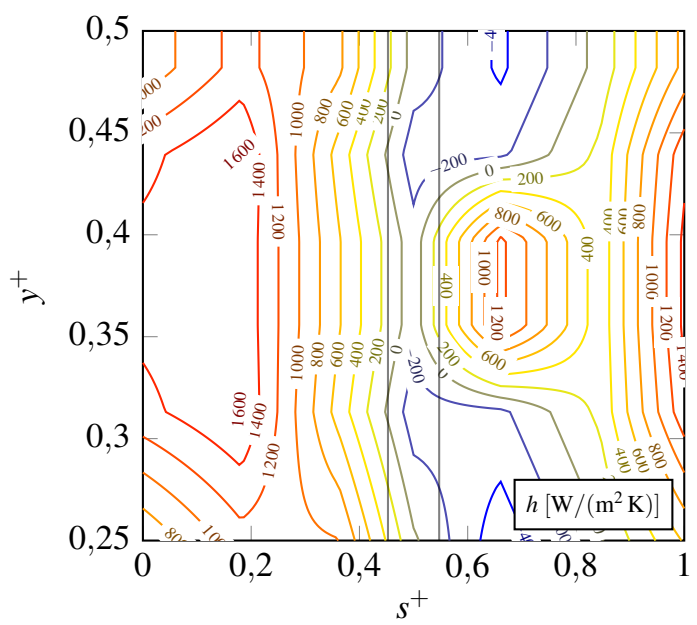

(f) BP114

Abbildung A.19: Ortsaufgelöste Wärmeübergangsmessungen für BP109 bis BP114 


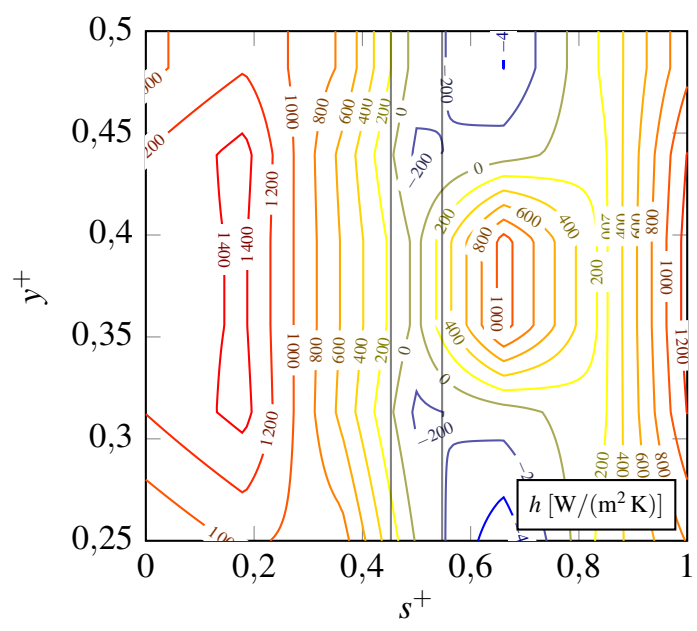

(a) BP115

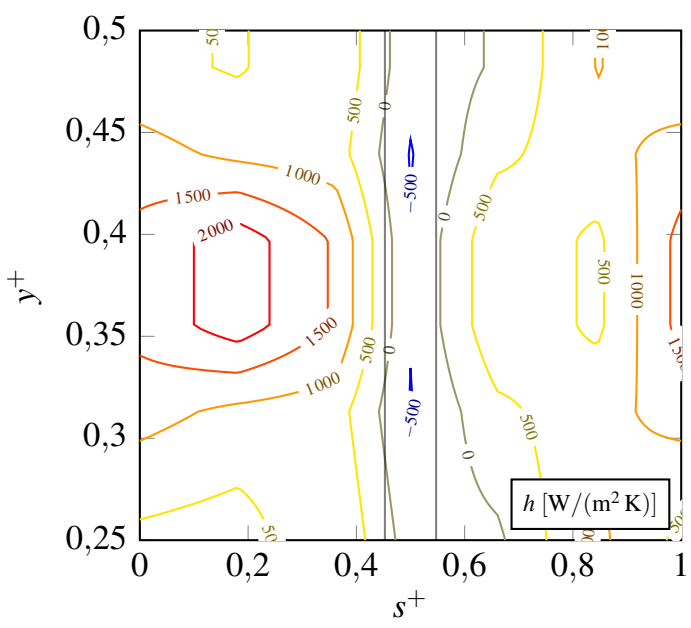

(c) BP117

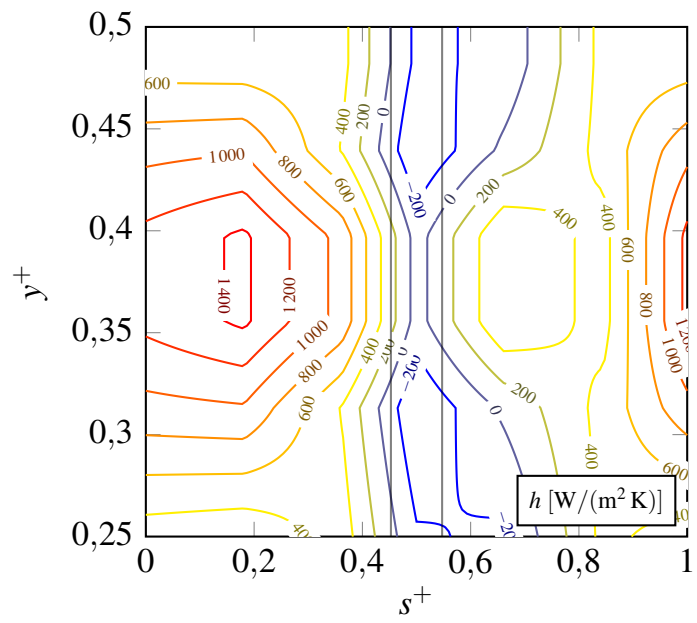

(e) BP119

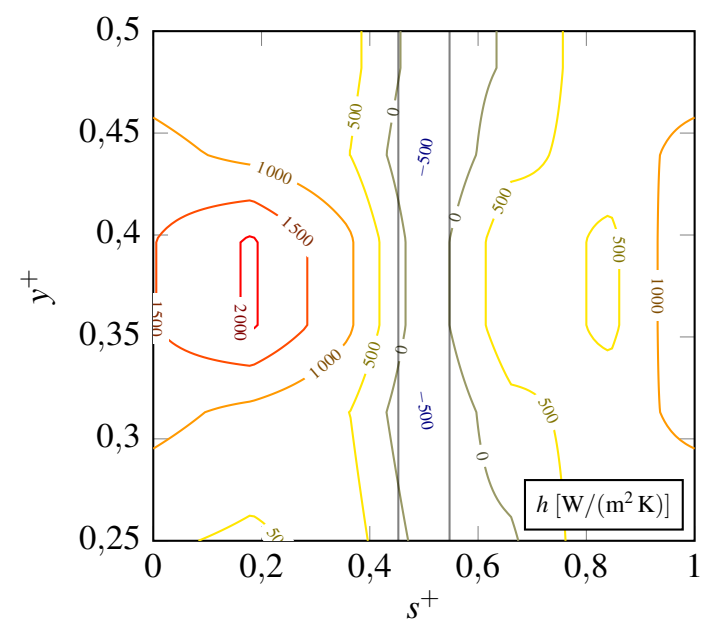

(b) BP116

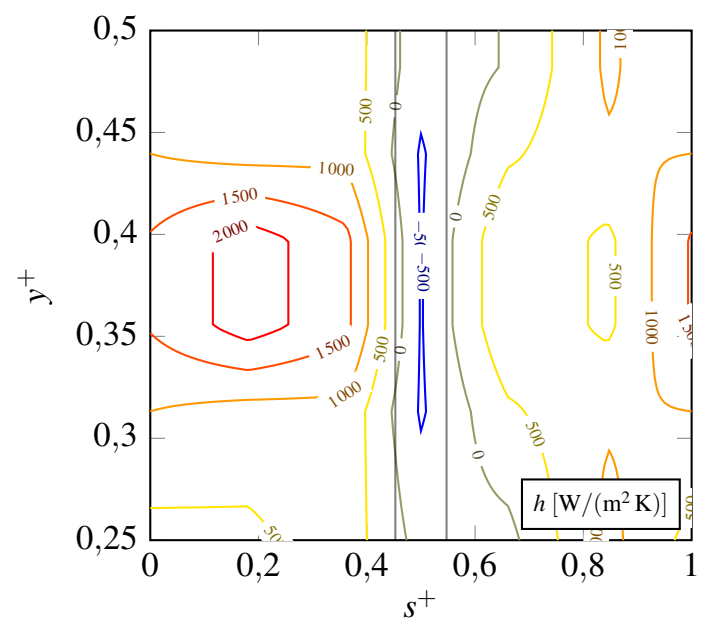

(d) BP118

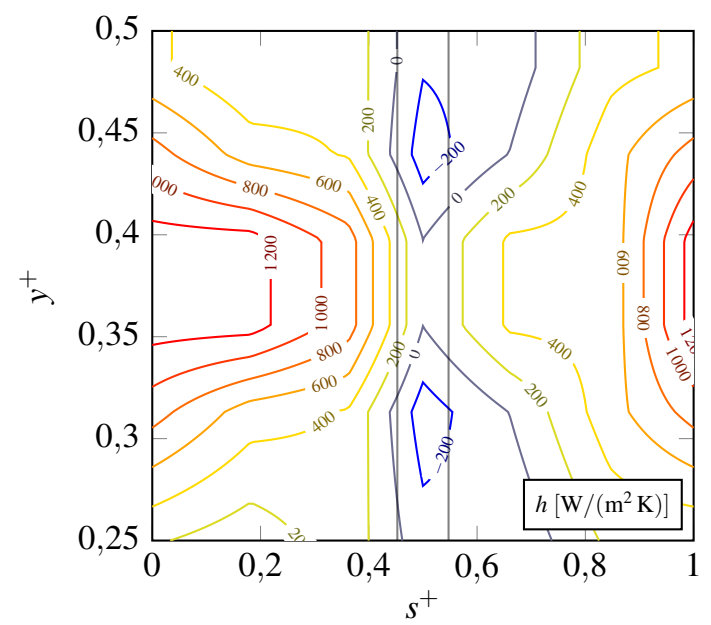

(f) BP120

Abbildung A.20: Ortsaufgelöste Wärmeübergangsmessungen für BP115 bis BP120 


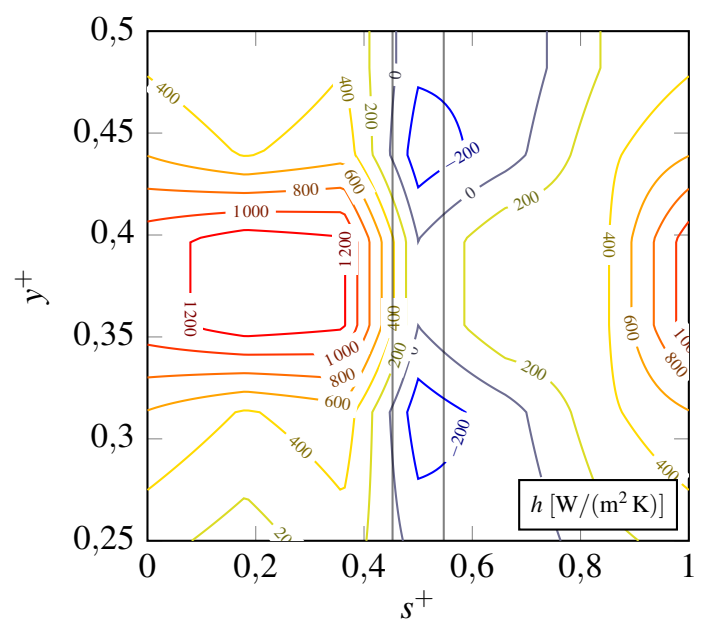

(a) BP121

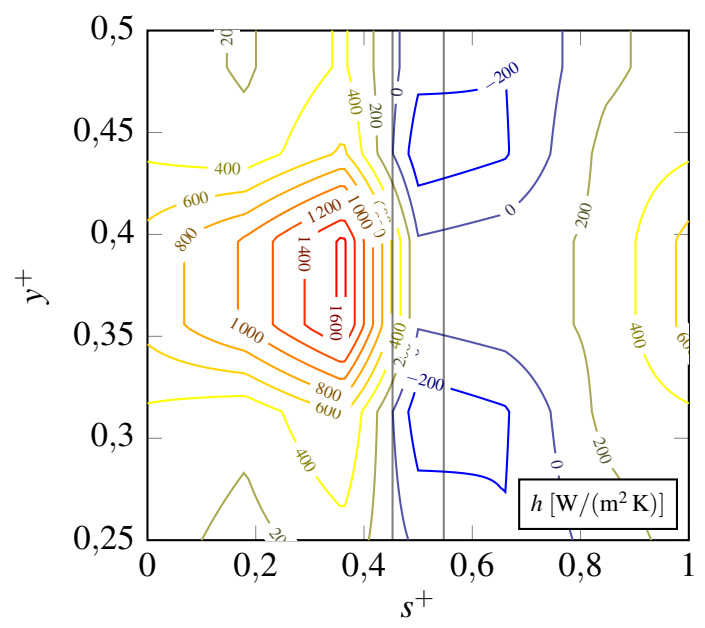

(c) BP123

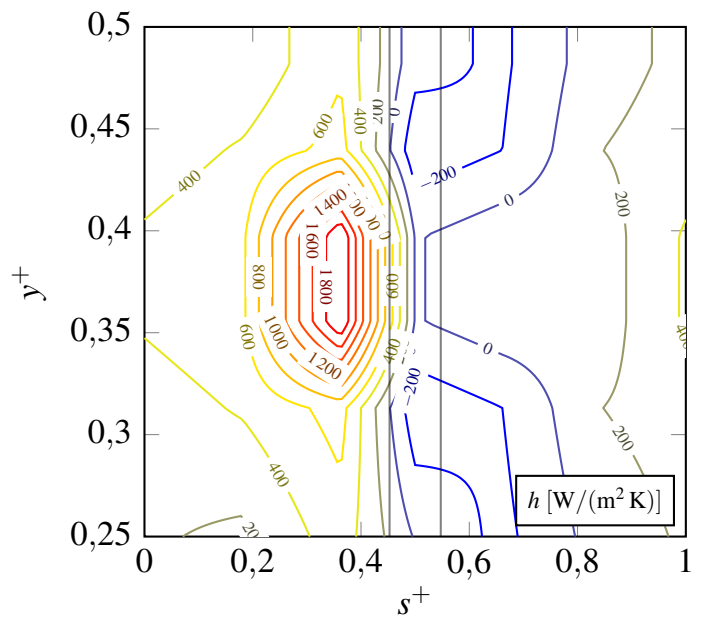

(e) BP125

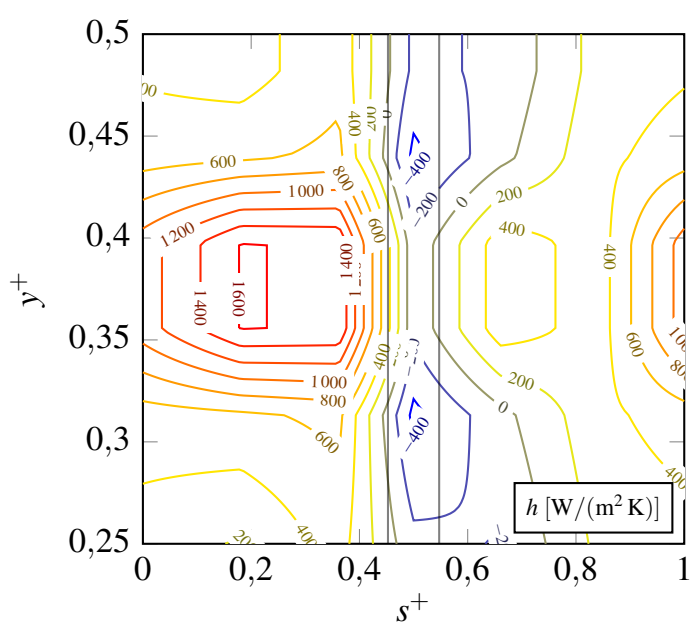

(b) BP122

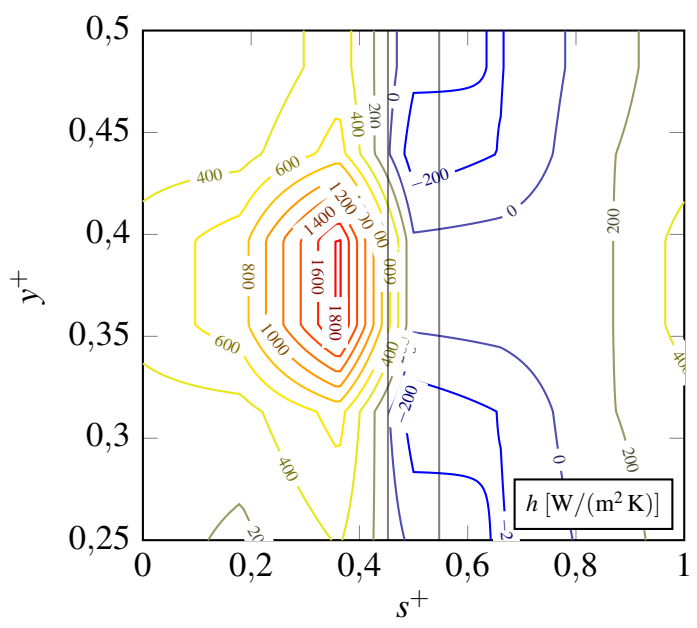

(d) BP124

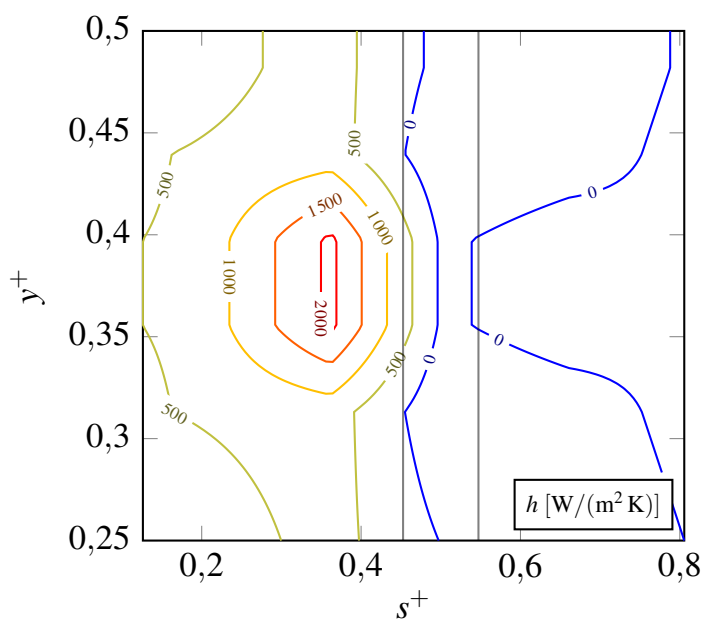

(f) BP126

Abbildung A.21: Ortsaufgelöste Wärmeübergangsmessungen für BP121 bis BP126 


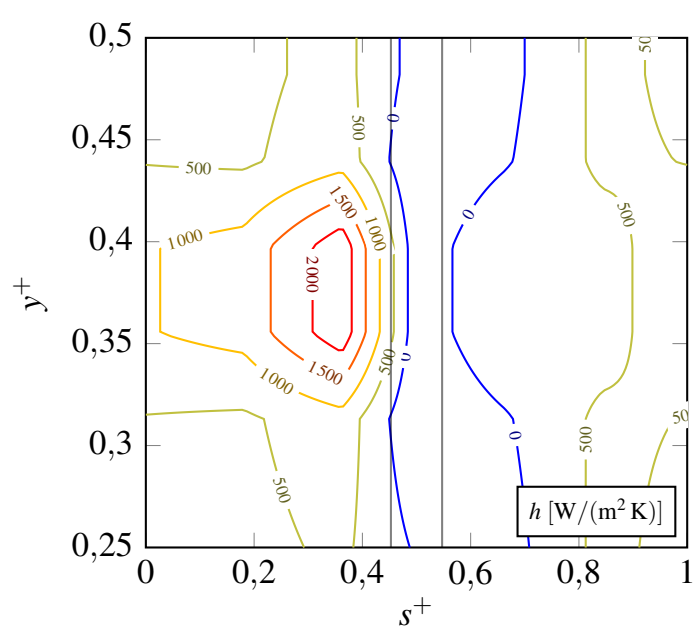

(a) BP127

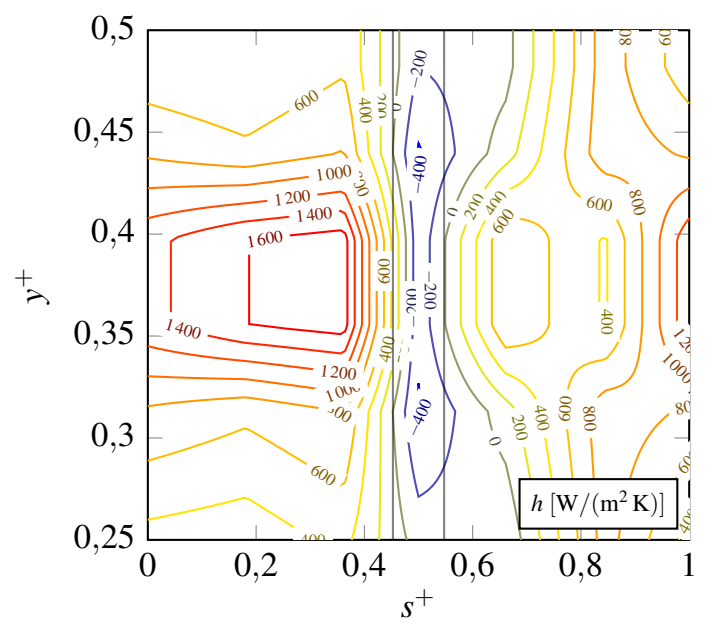

(c) BP129

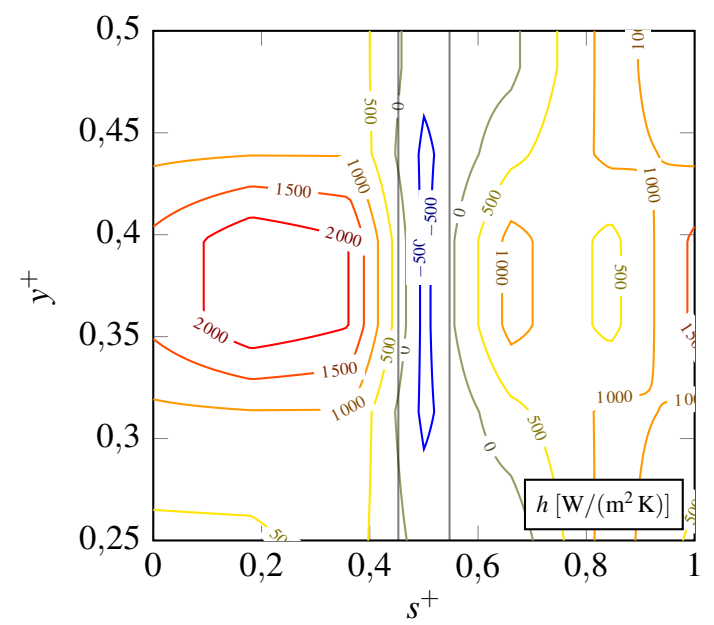

(b) BP128

Abbildung A.22: Ortsaufgelöste Wärmeübergangsmessungen für BP127 bis BP129 


\section{A.2 Stoffwertkorrelationen des verwendeten Öls}

Im Rahmen dieser Arbeit werden die von Glahn (1995) angegebenen Stoffwertkorelationen für Mobil Jet Oil II verwendet. In den angegebenen Zahlenwertgleichungen ist $\vartheta_{\text {s }}$ in K einzusetzen.

Kinematische Viskosität $v$ in $10^{-6} \mathrm{~m}^{2} / \mathrm{s}$ :

$$
\log \log (v+0,8)=8,962182-3,527033 \log \vartheta_{\mathrm{s}}
$$

Dichte $\rho$ in $\mathrm{kg} / \mathrm{m}^{3}$ :

$$
\rho=1329,44-1,16471 \vartheta_{\mathrm{s}}
$$

Wärmeleitfähigkeit $\lambda$ in $\mathrm{W} /(\mathrm{mK})$ :

$$
\lambda=0,15799-0,375 \cdot 10^{-4} \vartheta_{\mathrm{s}}
$$

Spezifische Wärme $c_{p}$ in $\mathrm{J} /(\mathrm{kg} \mathrm{K})$ :

$$
c_{p}=1054,86-2,88029 \vartheta_{\mathrm{s}}
$$

\section{A.3 Herleitung des spezifischen Relativimpulses}

Die Relativgeschwindigkeit $u_{\mathrm{r}}$, mit der das Öl auf die Zahnflanke auftrifft, kann durch ihren radialen Anteil $u_{\mathrm{r}, \mathrm{r}}$ und tangentialen Anteil $u_{\mathrm{r}, \mathrm{t}}$ berechnet werden:

$$
u_{\mathrm{r}}=\sqrt{u_{\mathrm{r}, \mathrm{r}}^{2}+u_{\mathrm{r}, \mathrm{t}}^{2}}
$$

Die Anteile sind jeweils die Summe der radialen und tangentialen Geschwindigkeitsanteile des Ölstrahls und des Zahns. Die Eigengeschwindigkeit des Zahns $u_{z}$ besitzt keine radiale Komponente. Somit wird Gleichung A.5 zu:

$$
u_{\mathrm{r}}=\sqrt{u_{\mathrm{s}, \mathrm{r}}^{2}+\left(u_{\mathrm{z}}-u_{\mathrm{s}, \mathrm{t}}\right)^{2}}
$$

Die radialen und tangentialen Anteile von $u_{\mathrm{s}}$ können durch den Düsenwinkel $\alpha$ ausgedrückt werden:

$$
u_{\mathrm{r}}=\sqrt{u_{\mathrm{s}}^{2} \cdot \cos ^{2} \alpha+\left(u_{\mathrm{z}}-u_{\mathrm{s}} \cdot \sin \alpha\right)^{2}} .
$$

Durch Umformen ergibt sich:

$$
\begin{aligned}
u_{\mathrm{r}} & =\sqrt{u_{\mathrm{z}}^{2}-2 u_{\mathrm{z}} u_{\mathrm{s}} \sin \alpha+\underbrace{u_{\mathrm{s}} \sin ^{2} \alpha+u_{\mathrm{s}}^{2} \cos ^{2} \alpha}_{\mathrm{s}}}=u_{\mathrm{s}}^{2} \\
& =\sqrt{u_{\mathrm{z}}^{2}-2 u_{\mathrm{z}} u_{\mathrm{s}}+u_{\mathrm{s}}^{2}+2 u_{\mathrm{z}} u_{\mathrm{s}}(1-\sin \alpha)} \\
& =\sqrt{\left(u_{\mathrm{z}}-u_{\mathrm{s}}\right)^{2}+2 u_{\mathrm{z}} u_{\mathrm{s}}(1-\sin \alpha)} .
\end{aligned}
$$


Durch Ersetzen durch $u_{\mathrm{s}}=\Sigma u_{\mathrm{z}}$ und Umformen wird Gleichung A.8 zu:

$$
\begin{aligned}
u_{\mathrm{r}} & =\sqrt{u_{\mathrm{z}}^{2}(1-\Sigma)^{2}+2 \Sigma u_{\mathrm{z}}^{2}(1-\sin \alpha)} \\
& =u_{\mathrm{z}} \sqrt{(1-\Sigma)^{2}+2 \Sigma-2 \Sigma(1-\sin \alpha)} \\
& =u_{\mathrm{z}} \sqrt{1+\Sigma^{2}-2 \Sigma(1-\sin \alpha)}
\end{aligned}
$$

und somit:

$$
\frac{u_{\mathrm{r}}}{u_{\mathrm{z}}}=\sqrt{1+\Sigma^{2}-2 \Sigma(1-\sin \alpha)}
$$




\section{Literaturverzeichnis}

Akin, L. S. und Townsend, D. P. (1985): Lubricant Jet Flow Phenomena in Spur and Helical Gears With Modified Center Distances and/or Addendums-For Out-of-Mesh Conditions. Journal of Mechanisms, Transmissions, and Automation in Design, Bd. 107, S. 24-30.

Akin, L. S., Mross, J. J. und Townsend, D. P. (1975): Study of Lubricant Jet Flow Phenomena in Spur Gears. Journal of Lubrication Technology, Bd. 97, S. 283. ISSN 00222305.

Andersson, M., Sosa, M. und Olofsson, U. (2017): Efficiency and temperature of spur gears using spray lubrication compared to dip lubrication. Proceedings of the Institution of Mechanical Engineers, Part J: Journal of Engineering Tribology, Bd. 231, S. 1390-1396. ISSN 1350-6501.

Arisawa, H., Nishimura, M., Imai, H. und Goi, T. (2014): Computational Fluid Dynamics Simulations and Experiments for Reduction of Oil Churning Loss and Windage Loss in Aeroengine Transmission Gears. Journal of Engineering for Gas Turbines and Power, Bd. 136, S. 092604. ISSN 0742-4795.

Barwell, F. T. (1958): Wear of Metals. Wear, Bd. 1, S. 317-332.

Benedict, G. H. und Kelley, B. W. (1961): Instantaneous Coefficients of Gear Tooth Friction. ASLE Transactions, Bd. 4, S. 59-70.

Birouk, M. und Lekic, N. (2009): Liquid Jet Breakup in Quiescent Atmosphere: a Review. Atomization and Sprays, Bd. 19, S. 501-528. ISSN 1046-5111.

Britton, R. D., Elcoate, C. D., Alanou, M. P., Evans, H. P. und Snidle, R. W. (2000): Effect of Surface Finish on Gear Tooth Friction. Journal of Tribology, Bd. 122, S. 354. ISSN 0742-4787.

Carper, H. J., Saavedra, J. J. und Suwanprateep, T. (1986): Liquid Jet Impingement Cooling of a Rotating Disk. Journal of Heat Transfer, Bd. 108, S. 540. ISSN 00221481.

Castro, J. und Seabra, J. (1998): Scuffing and lubricant film breakdown in FZG gears Part I. Analytical and experimental approach. Wear, Bd. 215, S. 104-113.

Chen, S.-J. und Tseng, A. A. (1992): Spray and jet cooling in steel rolling. International Journal of Heat and Fluid Flow, Bd. 13, S. 358-369.

DeWinter, A. und Blok, H. (1974): Fling-Off Cooling of Gear Teeth. Journal of Engineering for Industry, Bd. 96, S. 60. ISSN 00220817.

Diab, Y., Ville, F. und Velex, P. (2006a): Investigations on power losses in high-speed gears. In: Proceedings of the Institution of Mechanical Engineers, Bd. 220, S. 191-198.

Diab, Y., Ville, F. und Velex, P. (2006b): Prediction of Power Losses Due to Tooth Friction in Gears. Tribology Transactions, Bd. 49, S. 260-270. ISSN 1040-2004. 
Dorfman, L. A. (1963): Hydrodynamic resistance and the heat loss of rotating solids: Gidrodinamičeskoe soprotivlenie i teplootdača vraščajuščichsja tel. Oliver \& Boyd, Edinburgh.

Downs, S. J. und James, E. H. (1987): Jet impingement heat transfer - a literature survey. ASME, AIChE, and ANS, 24th National Heat Transfer Conference and Exhibition, Pittsburgh, PA, Aug. 9-12, 1987.

Dowson, D., Higginson, G. R. und Hopkins, D. W. (1977): Elasto-Hydrodynamic Lubrication: International Series on Materials Science and Technology, Bd. v.23 von International Series on Materials Science and Technology. Elsevier Science, Burlington, 2. Aufl. ISBN 978-0-08021302-6.

Durand de Gevigney, J., Changenet, C., Ville, F. und Velex, P. (2012): Thermal modelling of a back-to-back gearbox test machine: Application to the FZG test rig. Proceedings of the Institution of Mechanical Engineers, Part J: Journal of Engineering Tribology, Bd. 226, S. 501-515. ISSN 1350-6501.

Eastwick, C. N. und Johnson, G. (2008): Gear Windage: A Review. Journal of Mechanical Design, Bd. 130, S. 034001-1-034001-6. ISSN 10500472.

Ebadian, M. A. und Lin, C. X. (2011): A Review of High-Heat-Flux Heat Removal Technologies. Journal of Heat Transfer, Bd. 133, S. 110801. ISSN 00221481.

Faeth, G., Hsiang, L.-P. und Wu, P.-K. (1995): Structure and breakup properties of sprays. International Journal of Multiphase Flow, Bd. 21, S. 99-127. ISSN 03019322.

Fondelli, T., Andreini, A., Da Soghe, R., Facchini, B. und Cipolla, L. (2015): Numerical Simulation of Oil Jet Lubrication for High Speed Gears. International Journal of Aerospace Engineering, Bd. 2015, S. 1-13. ISSN 1687-5966.

Giffen, E. und Muraszew, A. (1953): The Atomisation of Liquid Fuels. Chapman \& Hall, London.

Glahn, A., Busam, S. und Wittig, S. (1997): Local and Mean Heat Transfer Coefficients Along the Internal Housing Walls of Aero Engine Bearing Chambers. ASME International Gas Turbine and Aeroengine Congress and Exhibition, June 2-5, 1997, Orlando, Florida, USA, S. V003T09A050.

Glahn, J. A. (1995): Zweiphasenströmungen in Triebwerkslagerkammern: Charakterisierung der Ölfilmströmung und des Wärmeübergangs. Dissertation, Universität Karlsruhe.

Gorse, P., Willenborg, K., Busam, S., Ebner, J., Dullenkopf, K. und Wittig, S. (2003): 3D-LDA Measurements in an Aero-Engine Bearing Chamber. In: ASME Turbo Expo, June 16-19, 2003, Atlanta, Georgia, USA, S. 257-265. ASME. ISBN 0-7918-3671-1.

Grubin, A. N. und Vinogradova, I. E. (1949): Investigation of the Contact of Machine Components. Central Scientific Research Institute for Technology and Mechanical Engineering (TsNIITMASh), Moskau. 
Guegan, J., Kadiric, A., Gabelli, A. und Spikes, H. (2016): The Relationship Between Friction and Film Thickness in EHD Point Contacts in the Presence of Longitudinal Roughness. Tribology Letters, Bd. 64, S. 248. ISSN 1023-8883.

Han, B. und Goldstein, R. J. (2001): Jet-impingement heat transfer in gas turbine systems. Annals of the New York Academy of Sciences, Bd. 934, S. 147-161.

Han, J.-C., Dutta, S. und Ekkad, S. (2013): Gas turbine heat transfer and cooling technology. CRC Press, Boca Raton, Fla., 2. Aufl. ISBN 9781439855683.

Handschuh, R. F. (1992): Effect of Lubricant Jet Location on Spiral Bevel Gear Operating Temperatures. International Power Transmission and Gearing Conference, September 13-16, 1992, Phoenix, AZ, USA, S. 275-282.

Handschuh, R. F. und Kicher, T. P. (1994): A Method for Thermal Analysis of Spiral Bevel Gears. Proceedings of the International Gearing Conference, September 7-9, 1994, Newcastle Upon Tyne, England, UK.

Hartnett, J. P. und Deland, E. C. (1961): The Influence of Prandtl Number on the Heat Transfer From Rotating Nonisothermal Disks and Cones. Journal of Heat Transfer, Bd. 83, S. 95. ISSN 00221481.

Hertz, H. (1881): Ueber die Berührung fester elastischer Körper. Journal für die reine und angewandte Mathematik, Bd. 92, S. 156-171.

Herwig, H. und Middelberg, G. (2008): The physics of unsteady jet impingement and its heat transfer performance. Acta Mechanica, Bd. 201, S. 171-184. ISSN 0001-5970.

Herwig, H., Mocikat, H., Gürtler, T. und Göppert, S. (2004): Heat transfer due to unsteadily impinging jets. International Journal of Thermal Sciences, Bd. 43, S. 733-741. ISSN 12900729.

Höhn, B.-R. und Michaelis, K. (2004): Influence of oil temperature on gear failures. Tribology International, Bd. 37, S. 103-109. ISSN 0301679X.

Höhn, B.-R., Michaelis, K. und Otto, H.-P. (2008): Influence of immersion depth of dip lubricated gears on power loss, bulk temperature and scuffing load carrying capacity. International Journal of Mechanics and Materials in Design, Bd. 4, S. 145-156. ISSN 1569-1713.

ISO (2014): ISO TR 15144:2014: Calculation of micropitting load capacity of cylindrical spur and helical gears - Part 1: Introduction and basic principles.

Jacobsen, K. (1987): Experimentelle Untersuchungen zum Durchfluss und Wärmeübergang in Durchblick- und Stufenlabyrinthdichtungen. Dissertation, Universität Karlsruhe.

Jane's Information Group (Hrsg.) (2005): Jane's all the world's aircraft. Jane Pub., London, 96th Aufl. ISBN 978-0710626844. 
Jie, P., Shaojun, L. und Xiaozhou, H. (2013): The Bulk Temperature Analysis of the Involute Spur Gear Based on Parameterized Modeling of APDL. Fifth International Conference on Measuring Technology and Mechatronics Automation, January 16-17, 2013, Hong Kong, China, S. 1146-1149.

Kanzow, M. E. (2017): Numerische Untersuchung der Strömung in einer Öleinspritzbrause unter Zentrifugaleinfluss. Bachelorarbeit, Karlsruher Institut für Technologie - Institut für Thermische Strömungsmaschinen, Karlsruhe.

Keller, M. C., Braun, S., Wieth, L., Chaussonnet, G., Dauch, T. F., Koch, R., Schwitzke, C. und Bauer, Hans-Jörg (2017): Smoothed Particle Hydrodynamics Simulation of OilJet Gear Interaction. Transactions of the American Society of Mechanical Engineers, S. V02BT41A019.

Kelley, B. W. und Lemanski, A. J. (1967): Lubrication of Involute Gearing. Proceedings of the Institution of Mechanical Engineers, Conference Proceedings, Bd. 182, S. 173-184. ISSN 0367-8849.

Klocke, F. und Brecher, C. (2017): Zahnrad- und Getriebetechnik: Auslegung - Herstellung Untersuchung - Simulation. Carl Hanser Verlag, München. ISBN 978-3-446-43140-9.

Kneer, J. (2017): Zur Interaktion Von Filmkühlung und Heißgasströmung Auf Konturierten Turbinenseitenwänden. Dissertation, Karlsruher Institut für Technologie.

Koffel, G., Ville, F., Changenet, C. und Velex, P. (2009): Investigations on the power losses and thermal effects in gear transmissions. Proceedings of the Institution of Mechanical Engineers, Part J: Journal of Engineering Tribology, Bd. 223, S. 469-479. ISSN 1350-6501.

Krantz, T. L. (2014): On the Correlation of Specific Film Thickness and Gear Pitting Life. American Gear Manufacturers Association, Alexandria, VA, USA. ISBN 978-1-61481-113-8.

Krug, M. (2018): Untersuchung der Ölversorgung in einer triebwerkstypischen Lagerkammer mittels experimenteller und numerischer Methoden. Dissertation, Karlsruher Institut für Technologie.

Krug, M. B., Peduto, D., Kurz, W. und Bauer, Hans-Jörg (2014): Experimental Investigation Into the Efficiency of an Aero Engine Oil Jet Supply System. In: Proceedings of the ASME Turbo Expo 2014, S. V01AT01A026. ASME. ISBN 978-0-7918-4557-8.

Kurz, W. (2018): VORLÄUFIG: Zweiphasenströmungen in Triebwerkslagerkammern und deren Absaugleitungen. Dissertation, Karlsruher Institut für Technologie.

Kurz, W. und Bauer, H.-J. (2014): An Approach for Predicting the Flow Regime in an Aero Engine Bearing Chamber. In: Proceedings of the ASME Turbo Expo 2014, S. V05CT16A037. ASME. ISBN 978-0-7918-4557-8. 
Lavalle, J. C., Rahman, M. M. und Kumar, A. (2007): Numerical analysis of heat transfer on a rotating disk surface under confined liquid jet impingement. International Journal of Heat and Fluid Flow, Bd. 28, S. 720-734.

Leoni, P. (1991): Hochleistungsgetriebe mit getrennter Schmierung und Kühlung: Zugl.: Stuttgart, Univ., Diss., 1991, Bd. 286 von Berichte des Institutes für Maschinenkonstruktion und Getriebebau. Inst. für Maschinenkonstruktion u. Getriebebau, Stuttgart. ISBN 3922823181.

Li, W. und Tian, J. (2017): Unsteady-state temperature field and sensitivity analysis of gear transmission. Tribology International, Bd. 116, S. 229-243. ISSN 0301679X.

Lienhard, J. H. V. (2006): Heat Transfer by Impingement of Circular Free-Surface Liquid Jets. 18th National \& 7th ISHMT-ASME Heat and Mass Transfer Conference, January 4-6, 2006, Guwahati, India.

Lin, S. P. und Reitz, R. D. (1998): Drop and Spray Formation from a Liquid Jet. Annual Review of Fluid Mechanics, Bd. 30, S. 85-105. ISSN 0066-4189.

Liu, X., Lienhard, J. H. V. und Lombara, J. S. (1991): Convective Heat Transfer by Impingement of Circular Liquid Jets. Journal of Heat Transfer, Bd. 113, S. 571-582.

Long, H., Lord, A. A., Gethin, D. T. und Roylance, B. J. (2003): Operating temperatures of oil-lubricated medium-speed gears: Numerical models and experimental results. Proceedings of the Institution of Mechanical Engineers, Part G: Journal of Aerospace Engineering, Bd. 217 , S. 87-106. ISSN 0954-4100.

Ma, C. F., Zhao, Y. H., Masuoka, T. und Gomi, T. (1996): Analytical study on impingement heat transfer with single-phase free-surface circular liquid jets. Journal of Thermal Science, Bd. 5, S. 271-277. ISSN 1003-2169.

Manin, L. und Play, D. (1999): Thermal Behavior of Power Gearing Transmission, Numerical Prediction, and Influence of Design Parameters. Journal of Tribology, Bd. 121, S. 693. ISSN 0742-4787.

Massini, D., Fondelli, T., Facchini, B., Tarchi, L. und Leonardi, F. (2016): High Speed Visualizations of oil Jet Lubrication for Aero-engine Gearboxes. Energy Procedia, Bd. 101, S. 1248-1255. ISSN 18766102.

Massini, D., Fondelli, T., Facchini, B., Tarchi, L. und Leonardi, F. (2017): Experimental Investigation on Power Losses due to Oil Jet Lubrication in High Speed Gearing Systems. Transactions of the American Society of Mechanical Engineers, S. V05BT15A030.

Michaelis, K., Höhn, B.-R. und Hinterstoißer, M. (2011): Influence factors on gearbox power loss. Industrial Lubrication and Tribology, Bd. 63, S. 46-55. ISSN 0036-8792.

Mizutani, H., Isikawa, Y. und Townsend, D. P. (1989): Effects of Lubrication on the Performance of High Speed Spur Gears. International Power Transmission and Gearing Conference, April 24-27, 1989, Chicago, Illinois, USA. 
Niemann, G. und Winter, H. (2003): Getriebe allgemein, Zahnradgetriebe - Grundlagen, Stirnradgetriebe, Bd. 2 von Maschinenelemente. Springer, Berlin, 2. Aufl. ISBN 3-54011149-2.

Ochs, M. (2011): Filmkühlung Transsonischer Turbinen: Infrarotthermographisches Messverfahren Zur Charakterisierung des Wärmeübergangs. Dissertation, Karlsruher Institut für Technologie.

Olver, A. V. (2002): Gear lubrication - a review. Proceedings of the Institution of Mechanical Engineers, Part J: Journal of Engineering Tribology, Bd. 216, S. 255-267. ISSN 1350-6501.

Patir, N. und Cheng, H. S. (1979): Prediction of the Bulk Temperature in Spur Gears Based on Finite Element Temperature Analysis. ASLE Transactions, Bd. 22, S. 25-36. ISSN 0569-8197.

Pinkus, O. (1990): Thermal Aspects of fluid film tribology. ASME Press, New York, 1. Aufl. ISBN 0-7918-0011-3.

Popiel, C. O. und Boguslawski, L. (1975): Local Heat-Transfer Coefficients on the Rotating Disk in Still Air. Int. J. Heat Mass Transfer, S. 167-171.

Pratt \& Whitney (2018): PW1100G-JM Product Card. https://www.pw.utc.com/products-andservices/products/commercial-engines/Pratt-and-Whitney-GTF-Engine/.

Pütz, F. (2018): Einfluss rauer Oberflächen auf den Wärmeübergang an einer TurbinenSeitenwand - experimentelle Untersuchung mit thermosensitiver Farbe. Dissertation, Karlsruher Institut für Technologie.

Reynolds, O. (1886): On the Theory of Lubrication and Its Application to Mr. Beauchamp Tower's Experiments, Including an Experimental Determination of the Viscosity of Olive Oil.. Proc. R. Soc. Lond., Bd. 40, S. 191-203.

Rotta, J. (1956): Experimenteller Beitrag zur Entstehung turbulenter Strömung im Rohr. Ingenieur-Archiv, Bd. 24, S. 258-281. ISSN 0020-1154.

Schober, H. (1983a): Einspritzschmierung bei Zahnradgetrieben. Techn. ber., Forschungsvereinigung Antriebstechnik, Frankfurt am Main.

Schober, H. (1983b): Untersuchungen zur Einspritzschmierung der Stirnradgetriebe: Zugl.: Stuttgart, Univ., Diss., 1983, Bd. 72 von Berichte des Institutes für Maschinenkonstruktion und Getriebebau. Inst. für Maschinenkonstruktion u. Getriebebau Univ, Stuttgart. ISBN 3922823041.

Schweitzer, P. H. (1937): Mechanism of Disintegration of Liquid Jets. Journal of Applied Physics, Bd. 8, S. 513-521. ISSN 0021-8979.

Seetharaman, S., Kahraman, A., Moorhead, M. D. und Petry-Johnson, T. T. (2009): Oil Churning Power Losses of a Gear Pair: Experiments and Model Validation. Journal of Tribology, Bd. 131, S. 022202. ISSN 0742-4787. 
Sheriff, H. S. und Zumbrunnen, D. A. (1994): Effect of Flow Pulsations on the Cooling Effectiveness of an Impinging Jet. Journal of Heat Transfer, Bd. 116, S. 886. ISSN 00221481.

Spikes, H. A. (1997): Mixed lubrication - an overview. Lubrication Science, Bd. 9, S. 221-253. ISSN 09540075.

Stevens, J. und Webb, B. W. (1991): Local Heat Transfer Coefficients Under an Axisymmetric, Single-Phase Liquid Jet. Journal of Heat Transfer, Bd. 113, S. 71-78.

Stripf, M. (2007): Einfluss der Oberflächenrauigkeit auf die transitionale Grenzschicht an Gasturbinenschaufeln: Experimentelle Untersuchungen und Entwicklung eines Berechnungsverfahrens. Dissertation, Universität Karlsruhe (TH).

Theißen, J. (2003): Graufleckenbildung an Zahnrädern: Ursachen, Prüfverfahren, Berechnung, Praxiserfahrungen. Techn. ber. Http://www.ib24.de/images/ibgregorius_PDFs/Vortraege/Vortragsmanuskript.pdf.

Tower, B. (1883): First Report on Friction Experiments. Proceedings of the Institution of Mechanical Engineers, Bd. 34, S. 632-659. ISSN 0020-3483.

Townsend, D. P. und Akin, L. S. (1981): Analytical and Experimental Spur Gear Tooth Temperature as Affected by Operating Variables. Journal of Mechanical Design, Bd. 103, S. 219-226.

Townsend, D. P. und Akin, L. S. (1983): Gear Lubrication and Cooling Experiment and Analysis. Journal of Mechanical Design, Bd. 103, S. 219-226.

VDI (2013): VDI-Wärmeatlas: Mit 320 Tabellen. VDI-Buch. Springer Vieweg, Berlin, 11th Aufl. ISBN 9783642199813.

von Kuensberg Sarre, C., Kong, S.-C. und Reitz, R. D. (1999): Modeling the Effects of Injector Nozzle Geometry on Diesel Sprays. SAE Transactions, Bd. 108, S. 1375-1388.

Wang, K. L. (1976): Thermal Elastohydrodynamic Lubrication of Spur Gears. Dissertation, Northwestern University, Illinois, USA.

Wang, Y., Niu, W., Chen, Y., Song, G. und Tang, W. (2016): Convection heat transfer and temperature analysis of oil jet lubricated spur gears. Industrial Lubrication and Tribology, Bd. 68, S. 624-631. ISSN 0036-8792.

Webb, B. W. und Ma, C.-F. (1995): Single-Phase Liquid Jet Impingement Heat Transfer. Advances in Heat Transfer, Bd. 26, S. 105-217.

Winter, H. und Michaelis, K. (1984): Scoring Load Capacity of Gears Lubricated with EP-Oils. Gear Technology.

Wu, P.-K., Miranda, R. F. und Faeth, G. M. (1994): Effects of initial flow conditions on primary breakup of nonturbulent and turbulent liquid jets. Proceedings of the American Institute of Aeronautics and Astronautics, Bd. 32. 
Wübbeler, G., Krystek, M. und Elster, C. (2008): Evaluation of measurement uncertainty and its numerical calculation by a Monte Carlo method. Measurement Science and Technology, Bd. 19, S. 084009. ISSN 0957-0233.

Wübbeler, G., Harris, P. M., Cox, M. G. und Elster, C. (2010): A two-stage procedure for determining the number of trials in the application of a Monte Carlo method for uncertainty evaluation. Metrologia, Bd. 47, S. 317-324. ISSN 0026-1394.

Xing, C. und Shaojun, L. (2013): Analysis of Bulk Temperature in High-Speed Gears Based on Finite Element Method. In: 2013 Fourth International Conference on Digital Manufacturing \& Automation (ICDMA), Qingdao, China, S. 202-206.

Zumbrunnen, D. A. (1992): Transient Convective Heat Transfer in Planar Stagnation Flows With Time-Varying Surface Heat Flux and Temperature. Journal of Heat Transfer, Bd. 114, S. 85. ISSN 00221481.

Zumbrunnen, D. A. und Aziz, M. (1993): Convective Heat Transfer Enhancement Due to Intermittency in an Impinging Jet. Journal of Heat Transfer, Bd. 115, S. 91. ISSN 00221481. 


\section{Studentische Abschlussarbeiten}

Folgende studentische Arbeiten haben zur Erzielung der Ergebnisse der vorliegenden Arbeit beigetragen:

Bauer, F. (2018): Thermalmodellierung des Sonnenrades eines Hochleistungsplanetengetriebes mit Öleinspritzkühlung. Masterarbeit, Karlsruher Institut für Technologie - Institut für Thermische Strömungsmaschinen. Betreuer: Bauer, H.-J., Mitbetreuer: von Plehwe, F. C.

Niehues, H. (2018): Unsicherheitsanalyse bei Wärmeübergangsuntersuchungen für ein Hochleistungsgetriebe durch die Monte-Carlo-Methode. Bachelorarbeit, Karlsruher Institut für Technologie - Institut für Thermische Strömungsmaschinen. Betreuer: Bauer, H.-J., Mitbetreuer: von Plehwe, F. C.

Thiery, F. (2018): Iterative Auswertung von Temperaturmessdaten zur Bestimmung des Wärmeübergangs an einem Höchstleistungszahnrad. Bachelorarbeit, Karlsruher Institut für Technologie - Institut für Thermische Strömungsmaschinen. Betreuer: Bauer, H.-J., Mitbetreuer: von Plehwe, F. C. 


\section{Curriculum Vitae}

Name

Geburtsdatum

Geburtsort

\section{Beruflicher Werdegang}

seit April 2020

Jan. 2019 - Mär. 2020

Feb. 2014 - Okt. 2018

Studium

Okt. 2006 - Mär. 2013
Felix von Plehwe

08. September 1987

Scherzingen, Schweiz

GE Aviation

Thermal System Dynamics

Lead Engineer

Cold Ray

Start-Up zur Entwicklung umweltfreundlicher Klimaanlagen

Co-Founder

Karlsruher Institut für Technologie

Institut für Thermische Strömungsmaschinen

Wissenschaftlicher Mitarbeiter

RWTH Aachen

Maschinenbau, Diplom

Vertiefungsrichtung Luft- und Raumfahrt

Schulbildung

2004 - 2006

Görres Gymnasium, Düsseldorf

$2003-2004$

Cranleigh School, Cranleigh, England

Ambrosius-Blarer Gymnasium, Gaienhofen 
Angesichts des hohen Nebenstromverhältnisses moderner Flugtriebwerke wird die Einführung eines Getriebes zwischen Niederdruckturbine und Fan attraktiv. Ein solches Getriebe stellt aufgrund der hohen übertragenen Leistung und den stark gegensätzlichen Forderungen nach geringem Gewicht, geringem Bauraum, hoher Zuverlässigkeit und hohem Wirkungsgrad eine große Herausforderung dar und erfordert die genaue Kenntnis aller beteiligten Vorgänge.

Einer dieser Vorgänge ist der Wärmeübergang durch Einspritzkühlung. In der vorliegenden Arbeit wird eine neuartige experimentelle Methode vorgestellt, mit der dieser am rotierenden Zahnrad ortsaufgelöst und mit hoher Genauigkeit bestimmt werden kann. Der Wärmeübergang wird mit dieser Methode für einen breiten Betriebsbereich ermittelt. Der Einfluss von Ölvolumenstrom, Drehzahl und Düsenwinkel auf den Wärmeübergang wird anhand dieser ortsaufgelösten Daten erstmalig beschrieben. Es zeigt sich die Existenz einer kritischen Drehzahl, bei deren Überschreitung ein deutlicher Rückgang des Wärmeübergangs auftritt. Durch Verwendung eines kinematischen Modells des Ölaufpralls können die beobachteten Zusammenhänge erklärt werden. Örtlich aufgelöste Messungen des Wärmeübergangs sind durch die vorliegende Arbeit erstmalig und für einen weiten Parameterbereich verfügbar. Dies versetzt den Entwickler in die Lage, eine ausreichende Kühlung der Zahnflanken sicherzustellen, ohne dabei Wirkungsgradverluste durch zu hohe eingesetzte Ölvolumenströme zu riskieren.

\section{Logos Verlag Berlin}

\section{ISBN 978-3-8325-5436-1}

ISSN 1615-4983 